\title{
Engineering Nanocomposite Membranes; Fabrication, Modification and Application
}

\author{
Al-Aani, Saif
}

How to cite:

Al-Aani, Saif (2018) Engineering Nanocomposite Membranes; Fabrication, Modification and Application. Doctoral thesis, Swansea University.

http://cronfa.swan.ac.uk/Record/cronfa40523

Use policy:

This item is brought to you by Swansea University. Any person downloading material is agreeing to abide by the terms of the repository licence: copies of full text items may be used or reproduced in any format or medium, without prior permission for personal research or study, educational or non-commercial purposes only. The copyright for any work remains with the original author unless otherwise specified. The full-text must not be sold in any format or medium without the formal permission of the copyright holder. Permission for multiple reproductions should be obtained from the original author.

Authors are personally responsible for adhering to copyright and publisher restrictions when uploading content to the repository.

Please link to the metadata record in the Swansea University repository, Cronfa (link given in the citation reference above.)

http://www.swansea.ac.uk/library/researchsupport/ris-support/ 


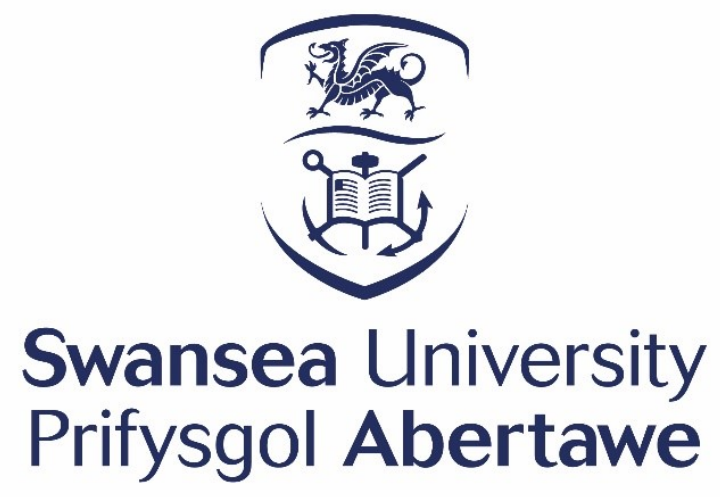

\title{
ENGINEERING NANOCOMPOSITE MEMBRANES; \\ FABRICATION, MODIFICATION AND APPLICATION
}

\author{
Saif Al Aani \\ B.Sc. (Chemical Engineering) (University of Baghdad, Iraq) \\ MTech (Chemical Engineering) (Guru Gobind Singh Indraprastha University, India)
}

Thesis submitted to the Swansea University in fulfilment of the requirements for the degree of Doctor of Philosophy

Swansea University

March 2018 


\section{Dedication}

I wish to dedicate this work to my parents (Ali Al Aani and Hanaa Alzuhairy), my wife (Daniah Alruhaily), and my children (Abathar, Sadan, and Maysarah) 


\section{Summary}

The engineering of novel membranes through fabrication and modification using engineered nanoscale materials (ENMs) presents tremendous opportunity within desalination and water treatment. This work presents an endeavour dedicated to investigate the design and fabrication of polymeric membranes and nanoscale materials. Also, to probe the role of nanoscale materials integration on the function of separating membranes aiming to diminish the propensity of the surface to foul.

In the first part of the work, an attempt was made to research and compare the potential of versatile UF membranes structures in terms of morphology, surface characteristics and performance. The potential performance of the hand-made fabricated (UF) membranes was systematically evaluated against three organic model foulants with dissimilar origins; humic acid (HA), sodium alginate (NaAlg), and bovine serum albumin (BSA), under different initial feed concentration and $\mathrm{pH}$ chemistry. A diverse range of surface characteristics and morphologies have been produced as a result of varying the dope casting solution concentration, which corresponds to the wide range of commercially available UF membranes $(6,10,35$ and $100 \mathrm{kDa})$. Also, a disparate fouling behaviour was observed depending on the membrane characteristics and the organic model foulant used. A one or more pore blocking mechanism were distinctly observed depending on the UF membrane cut-off used.

Subsequently, the research presented the development of a novel nanocomposite membrane incorporating antimicrobial nanoparticles which have the potential to lower membrane biofouling. Antibacterial hybrid nanostructures (HNS) comprising of Ag decorated MWCNTs were successfully synthesised with the assistance of microwave irradiation. The HNS were then employed to fabricated antibacterial nanocomposite membranes via the classical phase inversion technique in order to assess their antimicrobial properties against two bacterial species; E. coli and $S$. aureus. The nanocomposite membranes remarkably displayed antibacterial activity (4.24 and 2.9 log kill) against the two species respectively. A higher stability under crossflow conditions was also demonstrated.

Finally, for desalination applications, novel HNS comprising of a mussel-inspired PDA coated M/MO-MWCNTs, were successfully synthesised and used to fabricate TFN membranes. For comparison, four different $\mathrm{M} / \mathrm{MO}\left(\mathrm{Al}_{2} \mathrm{O}_{3}, \mathrm{Fe}_{2} \mathrm{O}_{3}, \mathrm{TiO}_{2}\right.$ and $\left.\mathrm{Ag}\right)$ nanoparticles (NPs) were in situ synthesised/loaded on the surface of CNTs, and the resultant HNS were further coated with a thin polymeric film of PDA. An intermediate layer of the HNS was then deposited on a PES substrate membrane, and an interfacial polymerisation (IP) process was carried out to render a polyamide (PA) thin layer above the intermediate layer. Both HNS and TFN were characterised using different characterisation tools, and the performance of nanofiltration (NF) membranes was evaluated against monovalent, divalent salts and heavy metal solutions. The fabricated TFN-NF membranes had higher performance in terms of their permeation characteristics compared to the thin film composite TFC membrane ( 9.6-11.6 LMH), while maintaining their selectivity $(\geq 91 \%)$ against both monovalent and divalent salts solutions, and $(>92 \%)$ against the multi-component heavy metal solution. The experimental results disclosed a high retention capability for TFC and TFN membranes along with greater potential stability/compatibility within the polymeric PA matrix. This implies that the NF membranes fabricated in this work can be employed for water reclamation purposes. 


\section{Declaration}

This work has not previously been accepted in substance for any degree and is not being concurrently submitted in candidature for any degree.

Signed:

(Candidate)

Date:

\section{STATEMENT 1}

This thesis is the result of my own investigations, except where otherwise stated. Other sources are acknowledged by footnotes giving explicit references. A bibliography is appended.

Signed:

(Candidate)

Date:

Signed:

(Supervisor)

Date:

\section{STATEMENT 2}

I hereby give consent for my thesis, if accepted, to be available for photocopying and for interlibrary loan, and for the title and summary to be made available to outside organizations.

Signed:

(Candidate)

Date: 
The following publications have been based on this work:

1- Al Aani, S., Wright, C. J., Atieh, M. A., \& Hilal, N. (2017). Engineering nanocomposite membranes: Addressing current challenges and future opportunities. Desalination, 401, 1-15.

2- Oatley-Radcliffe, D. L., Al-Aani, S., Williams, P. M., \& Hilal, N. (2017). Mass Transport in Porous Liquid Phase Membranes. Membrane Characterization, 337.

3- Thomas, T. E., Al Aani, S., Oatley-Radcliffe, D. L., Williams, P. M., \& Hilal, N. (2017). Laser Doppler Electrophoresis and electro-osmotic flow mapping: A novel methodology for the determination of membrane surface zeta potential. Journal of Membrane Science, 523, 524-532.

4- Al Aani, S., Gomez, V., Wright, C. J., \& Hilal, N. (2017). Fabrication of antibacterial mixed matrix nanocomposite membranes using hybrid nanostructure of silver coated multi-walled carbon nanotubes. Chemical Engineering Journal, $326,721-736$.

5- Al Aani, S., Haroutounian, A., Wright, C. J., \& Hilal, N. (2018). Thin Film Nanocomposite (TFN) membranes modified with polydopamine coated metals/carbon-nanostructures for desalination applications. Desalination, 427, 6074.

6- Al Aani, S., Wright, C. J., \& Hilal, N. (2018). Investigation of UF membrane fouling and potentials as a pre-treatment step in desalination and surface water applications. Desalination, 432, 115-127. 


\section{Acknowledgements}

This thesis would not become a reality without the kind and the limitless support of many individuals. To all of them, I would like to present my sincere gratitude.

Foremost, I would like to extend my special thanks to my supervisor, Professor Nidal Hilal, the one who served not only as a supervisor but also as my inspiration in order to pursue this undertaking. Nevertheless, my co-supervisor, Dr. Chris Wright, for his constant supervision and guidance throughout my entire Ph.D. journey. I am highly indebted for imparting their expertise and knowledge in this study. Special thanks to Daniel Johnson and Virginia Gómez for their kind support and contribution in this work.

To my parents for their encouragement and support which helped me in fulfilment of this paper. My beloved wife and lovable children.

Also, many thanks and appreciations to my people and colleagues who have willingly helped me out in completing this endeavour.

Finally, I would like to express my gratitude for financial support received from The Higher Committee for Education Development in Iraq (HCED) for providing my scholarship. 


\section{Contents}

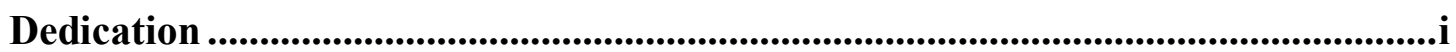

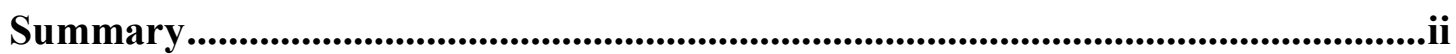

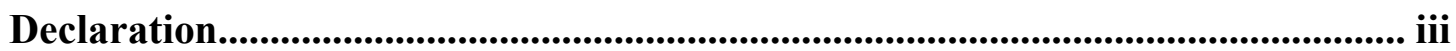

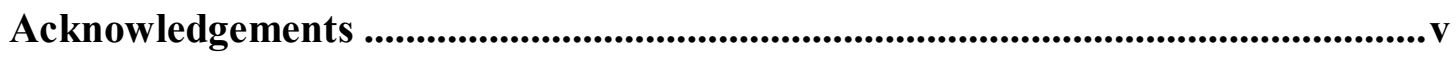

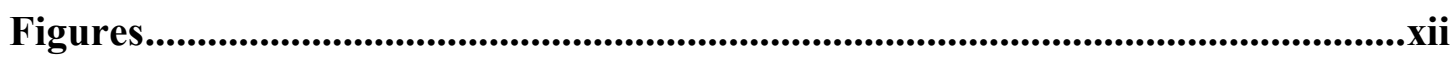

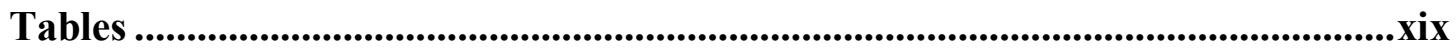

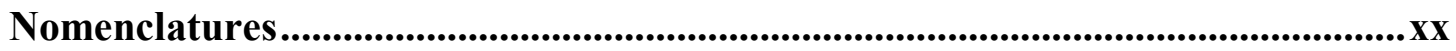

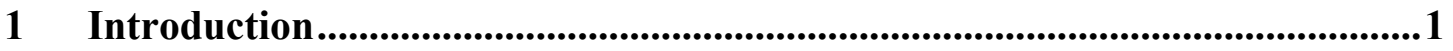

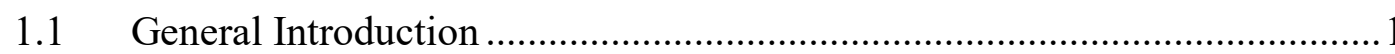

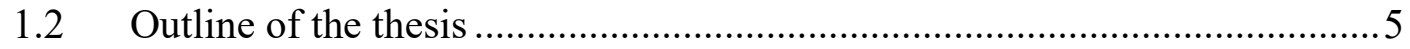

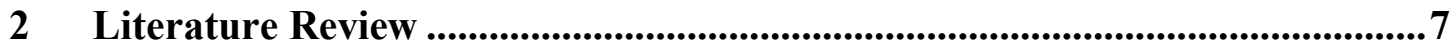

2.1 An overview of membrane separation processes .....................................

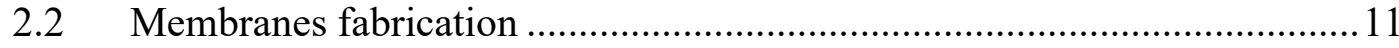

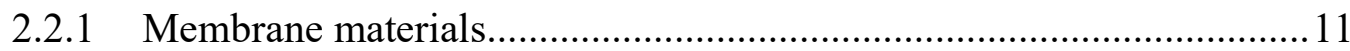

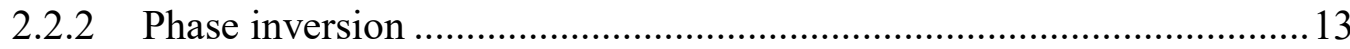

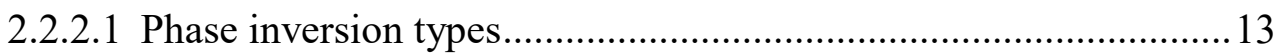

2.2.2.2 Parameters affecting phase inversion process. ............................. 14

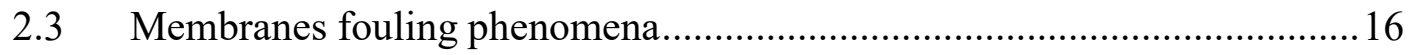

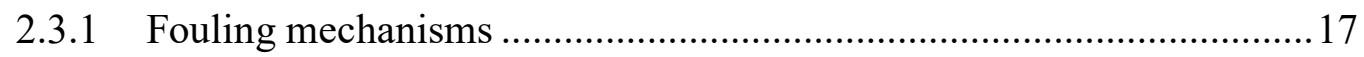

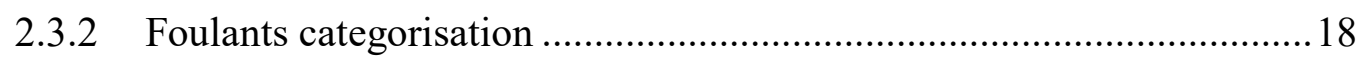

2.3.3 Factors affecting membranes fouling............................................20

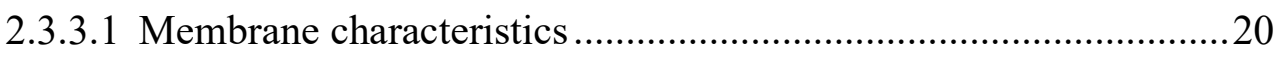

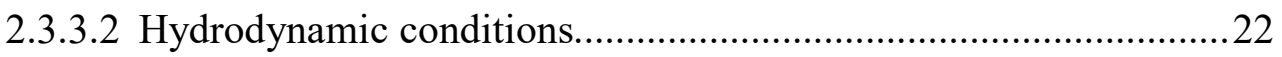

2.3.3.3 Feed solution characteristics.....................................................2 23 


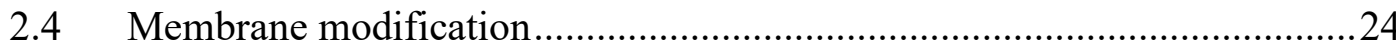

2.4.1 ENMs for polymeric membrane applications. ....................................25

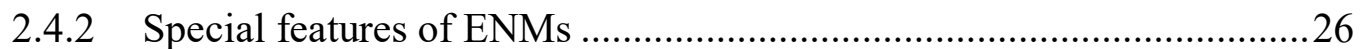

2.4.3 Strategies for incorporating ENMs into polymeric membranes ...........29

2.4.4 Influence of various ENMs on morphological and antifouling properties

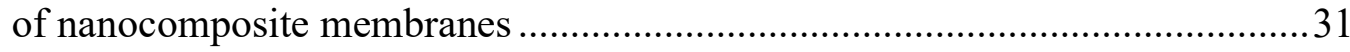

2.4.4.1 Metal/metal oxides based nanoparticles ..................................... 31

2.4.4.2 Carbon-based nanomaterials..................................................... 41

2.4.4.3 Cellulose-based nanoscale materials ............................................46

2.4.5 Issues arising from the incorporation of ENMs into polymeric membranes 49

2.4.6 Future improvements for ENMs incorporation into polymeric membranes 50

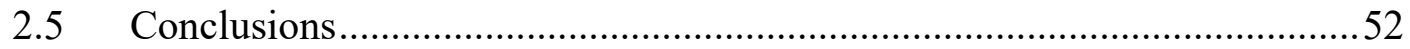

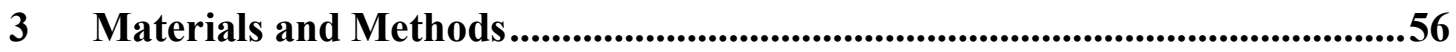

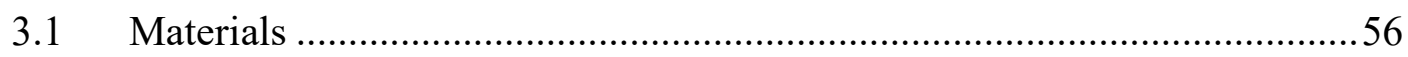

3.1.1 Materials for membrane fabrication......................................................56

3.1.2 Materials for hybrid nanostructures fabrication................................57

3.1.3 Materials for characterisation and fouling tests .................................57

3.2 Analytical and measuring instruments .................................................5

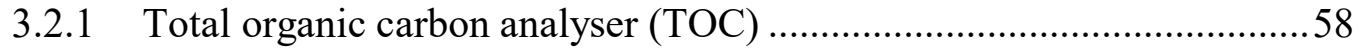

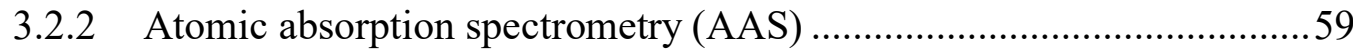

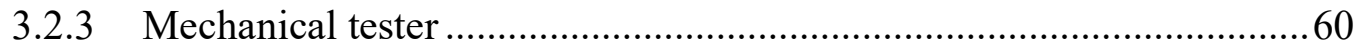

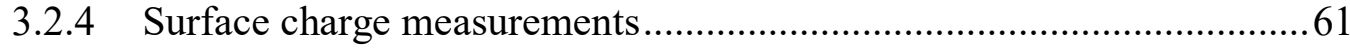

3.2.4.1 Membranes surface charge techniques ....................................... 61

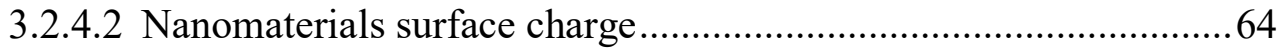

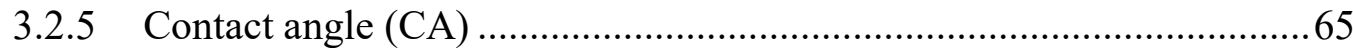




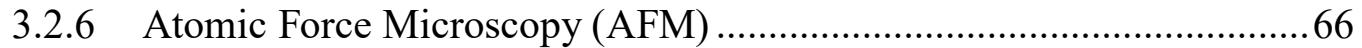

3.2.7 Scanning Electron Microscope (SEM) and (EDX) .............................6 68

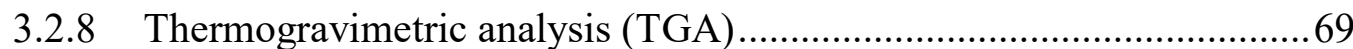

3.2.9 Fourier transform infrared spectrophotometry (FT-IR) .......................69

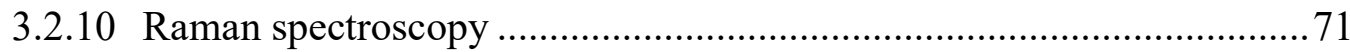

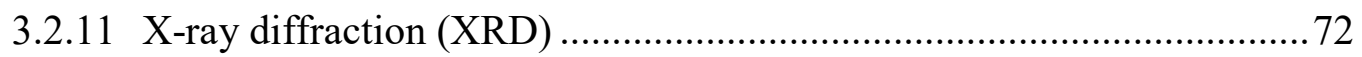

3.2.12 X-ray photoelectron spectroscopy (XPS) …..................................... 72

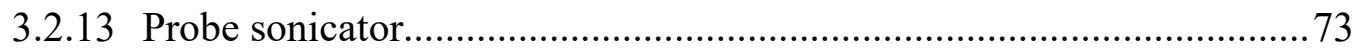

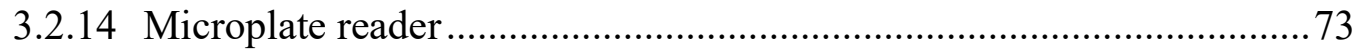

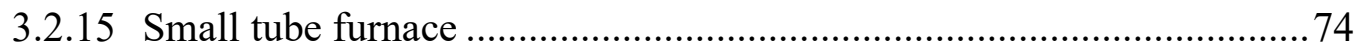

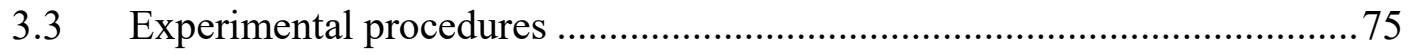

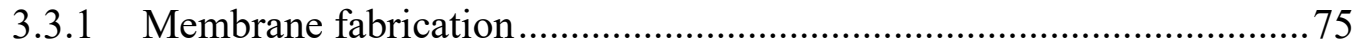

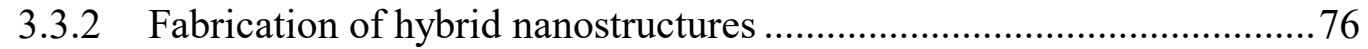

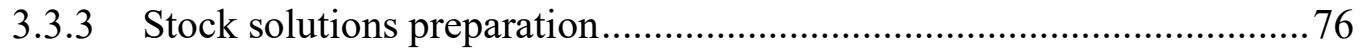

3.3.4 Permeability, retention and fouling measurements............................. 77

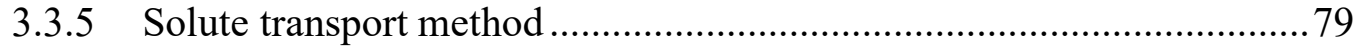

4 Investigation of Polyethersulfone polymer concentration on UF membrane characteristics and performance; a comprehensive study ....................................83

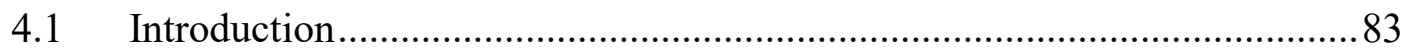

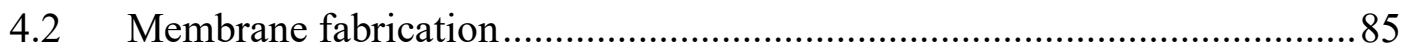

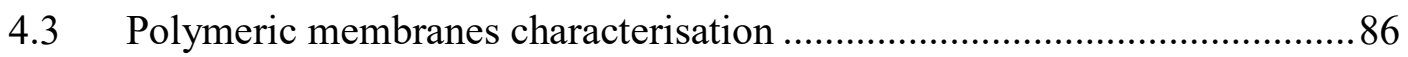

4.3.1 Cross-sectional analysis by scanning electron microscope (SEM)........86

4.3.2 Surface morphology analysis by atomic force microscope (AFM) .......87

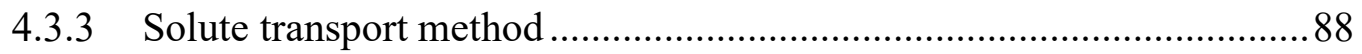

4.3.4 Zeta potential of membranes.......................................................... 90

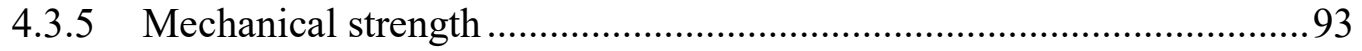


4.3.6 Pure water flux and hydrophilicity/hydrophobicity of membranes .......95

4.4 Evaluation of membranes performance with organic foulants ....................96

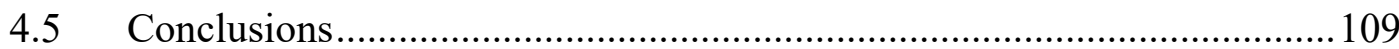

5 Fabrication and characterisation of versatile metal/metal oxide - carbon based hybrid nanostructures. ........................................................................................112

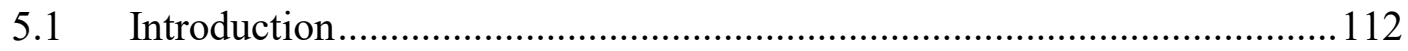

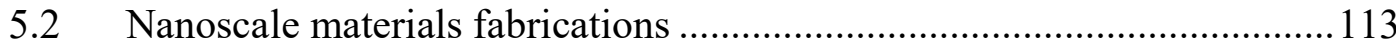

5.2.1 Purification and carboxylation of MWCNTs....................................113

5.2.2 Decoration of MWCNTs with M/MO nanoparticles .........................114

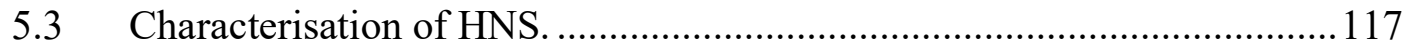

5.3.1 FTIR spectra evaluation of MWCNTs...........................................117

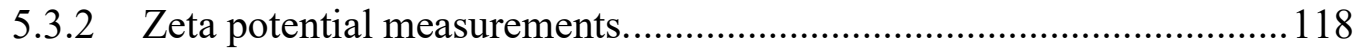

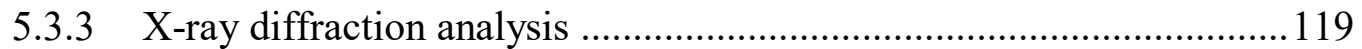

5.3.4 Microscopic observation and elemental composition.........................122

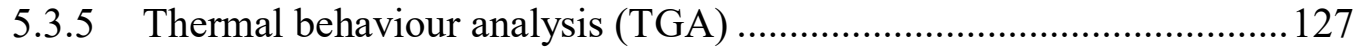

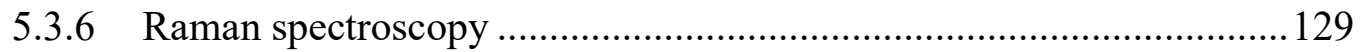

5.3.7 X-ray photoelectron spectroscopy (XPS) ..........................................134

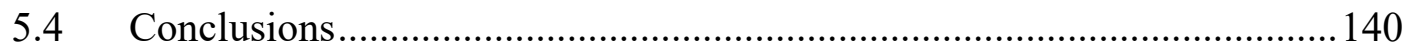

6 Fabrication of antibacterial nanocomposite membranes enabled by hybrid nanostructure modulated by microwave irradiation.................................................141

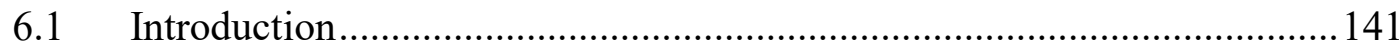

6.2 Synthesis of hybrid nanostructured materials ........................................ 144

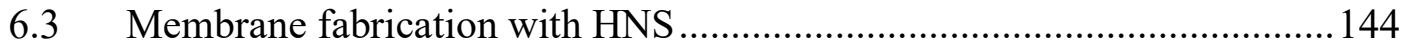

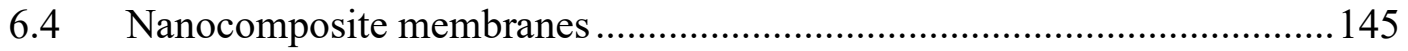

6.4.1 Cross section morphology of nanocomposites ................................. 146

6.4.2 Static contact angle and hydrophilicity measurements ...................... 147 
6.4.3 Mean pore size and pore size distribution determination 149

6.4.4 Surface zeta potential of nanocomposite membranes 151

6.4.5 Pure water flux 152

6.5 Potential bacteriostatic activities of HNS and nanocomposites 153

6.5.1 Bacteriostatic of HNS 153

6.5.2 Bacteriostatic activities of nanocomposite membranes 155

6.6 Morphological characterisation of microorganisms

6.7 Conclusions

7 Thin Film Nanocomposite (TFN) membranes modified with polydopamine coated metals/carbon-nanostructures for desalination applications....................164

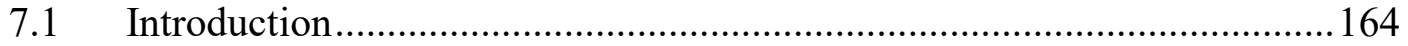

7.2 Synthesis of hybrid nanostructured materials ........................................ 167

7.3 Fabrication of thin film nanocomposite (TFN) ....................................... 168

7.4 Characterization of PDA coated nanocomposite materials .......................169

7.4.1 FTIR spectra evaluation of MWCNTs .............................................169

7.4.2 Thermal behaviour analysis (TGA) .............................................. 170

7.4.3 Zeta potential measurements............................................................... 171

7.5 Characterisation of thin film nanocomposite membranes. ........................ 172

7.5.1 Infrared spectra (FT-IR) of membranes.......................................... 172

7.5.2 Morphology of TFN membranes ...................................................... 174

7.5.3 Atomic force microscope (AFM) ................................................... 176

7.5.4 Static contact angle and hydrophilicity measurements .....................178

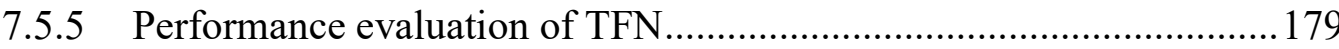

7.5.5.1 Monovalent and divalent salts ................................................. 179

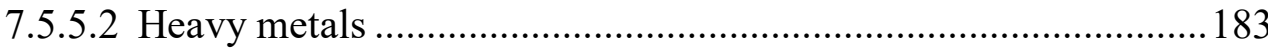

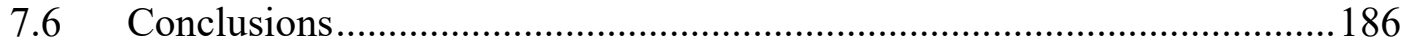




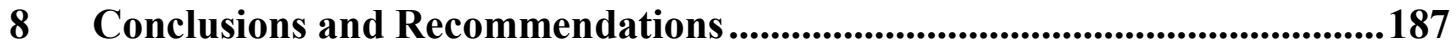

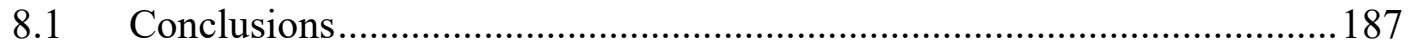

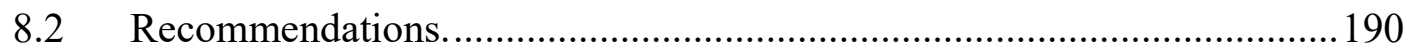

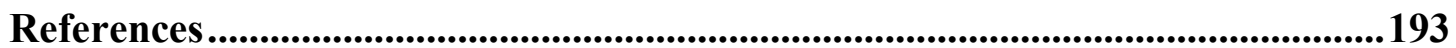




\section{Figures}

Figure 2.1: Classification of membrane separation processes, (Donnelly et al., 1998).

Figure 2.2: Schematic diagrams of the principal types of membrane (Baker, 2004) .. 11

Figure 2.3: Fouling mechanisms; (A) complete blocking, (B) intermediate blocking,

(C) standard blocking, and (D) cake blocking. .18

Figure 2.4: SEM and TEM images for various zero-dimensional (0D) ENMs. (A) Quantum dots, (B) nanoparticles arrays, (C) core-shell nanoparticles, (D) hollow cubes and (E) nanospheres. Adopted from (Tiwari et al., 2012).

Figure 2.5: SEM and TEM images for various one-dimensional (1D) ENMs. (A) Nanowires, (B) nanorods, (C) nanotubes, (D) nanobelts, (E) nanoribbons, and (F)hierarchical nanostructures. Adopted from (Tiwari et al., 2012). .27

Figure 2.6: SEM and TEM images for various two-dimensional (2D) ENMs (A) Junctions (continuous islands), (B) branched structures, (C) nanoplates, (D) nanosheets, (E) nanowalls, and (F) nanodisks Adopted from (Tiwari et al., 2012). .28

Figure 2.7: SEM and TEM images for various three-dimensional (3D) ENMs. (A) Nanoballs, (B) nanocoils, (C) nanocones, (D) nanopillers and, (E) nanoflowers.

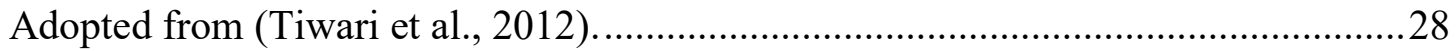

Figure 2.8: Comparison of FE-SEM images (A) simultaneous electrospin/electrospray, (B) LBL, and (C) grafting (Mauter et al., 2011).... .30

Figure 2.9: SEM images of silver nanoparticles incorporated into electrospun membrane filters (PEO). The right-hand image demonstrates the incorporation of triangular silver nanoparticles. .32

Figure 2.10: SEM images of CNTs. The left image presents CNTs (scale bar 20nm), the right image presents Fe-CNTs (scale bar 50nm). 42

Figure 2.11: Arrangement of graphene sheets to produce SWCNTs, DWCNTs, and MWCNTs respectively (from left to right). 43 
Figure 2.12: AFM images of dried cellulose nanocrystals (left), and fabricated membrane impregnated with $4 \%$ cellulose nanocrystals (right). Adopted from (AlMalek, 2012)

Figure 2.13: Different functional groups attached to iron oxide NPs surface (Dias et al., 2011) .52

Figure 3.1: Total organic carbon analyzer (TOC-L, Shimadzu) .59

Figure 3.2: Atomic absorption spectrometer (PinnAAcle 900F). .59

Figure 3.3: Instron - universal tensile tester instrument. .60

Figure 3.4: Electrokinetic Analyzer (EKA) (Anton- Paar GmbH, Austria) .62

Figure 3.5: Surface zeta potential cell (ZEN1020). .63

Figure 3.6: Malvern Zetasizer ZN nanoseries instrument (Malvern Instruments Ltd, Malvern, UK)

Figure 3.7: Universal dip cell kit (ZEN1002)

Figure 3.8: VCAoptima contact angle instrument, (AST Products, MA - USA). .65

Figure 3.9: Atomic force microscopy (AFM), (Bruker, USA). .66

Figure 3.10: Schematic demonstration of the principle of AFM operation. .67

Figure 3.11: (A) Scanning electron microscopy (SEM S4800- Hitachi), and (B) sputter coater machine (Quorum, Q150T ES). .68

Figure 3.12: Thermal gravimetric analysis (TGA) - TA instrument (Q600SDT). .69

Figure 3.13: FTIR - Spectrum Two - (PerkinsElmer, USA) ...................................... 70

Figure 3.14: Basic component in FT-IR spectrometer........................................... 71

Figure 3.15: InVia ${ }^{\mathrm{TM}}$ Confocal Raman Microscope (Renishaw)................................ 71

Figure 3.16: Brüker d8 DISCOVER diffractometer............................................... 72

Figure 3.17: X-ray photoelectron spectrometer (Kratos, AXIS SUPRA). ..................73

Figure 3.18: Tip sonicator (Vibra cellTM - Sonics, USA) ....................................... 73

Figure 3.19: Microplate reader (Synergy H1 hybrid, BMW Labtech). .......................74

Figure 3.20: High-temperature tube furnace (Carbolite Gero, UK). .......................... 74 
Figure 3.21: Dope casting solution (PES/NMP/PVP) ............................................. 75

Figure 3.22: Automated casting knife (RK film applicator)................................... 76

Figure 3.23: Schematic description of the cross-flow NF filtration unit .....................78

Figure 4.1: SEM cross-section images for; (A) PES16, (B) PES18, (C) PES20, and (D)

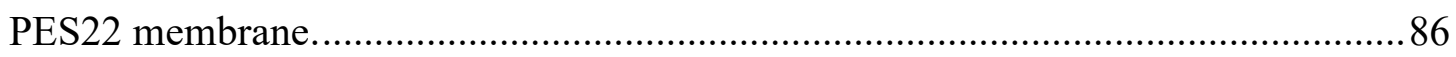

Figure 4.2: (1 $\mu \mathrm{m} \times 1 \mu \mathrm{m})$ AFM images for; (A) PES16, (B) PES18, (C) PES20 and (D) PES22 membrane. .88

Figure 4.3: Solute separation curve plotted on log-normal probability paper (LN3 software).

Figure 4.4: Probability density function curves of UF/PES membranes .90

Figure 4.5: (A) Measured electrophoretic mobility of tracer particles as a function of displacement from the membrane surface and (B) phase plots at four different displacements from the membrane surface. 91

Figure 4.6: Surface zeta potential profile for the UF membranes as a function of $\mathrm{pH}$, determined by LDE. .92

Figure 4.7: Surface zeta potential profile for the UF membranes as a function of $\mathrm{pH}$, determined by TSP. .93

Figure 4.8: Influence of dope casting solution concentration on strain-stress curves relationship of membranes. .95

Figure 4.9: PWF and contact angle measurements for membranes .96

Figure 4.10: Influence of solution $\mathrm{pH}$ on HA rejection coefficients (A), and fouling behaviour of (B) PES22, (C) PES20, (D) PES18, and (E) PES16 membrane. .99

Figure 4.11: Influence of solution initial feed concentration on HA rejection coefficients (A), and fouling behaviour of (B) PES22, (C) PES20, (D) PES18, and (E) PES16 membrane. 100

Figure 4.12: Influence of solution $\mathrm{pH}$ on $\mathrm{NaAlg}$ rejection coefficients (A), and fouling behaviour of (B) PES22, (C) PES20, (D) PES18, and (E) PES16 membrane. 102 
Figure 4.13: Influence of solution initial feed concentration on NaAlg rejection coefficients (A), and fouling behaviour of (B) PES22, (C) PES20, (D) PES18, and (E) PES16 membrane.

Figure 4.14: Influence of solution $\mathrm{pH}$ on BSA rejection coefficients (A), and fouling behaviour of (B) PES22, (C) PES20, (D) PES18, and (E) PES16 membrane. 106

Figure 4.15: Influence of solution initial feed concentration on BSA rejection coefficients (A), and fouling behaviour of (B) PES22, (C) PES20, (D) PES18, and (E) PES16 membrane. 107

Figure 5.1: Reflux unit for functionalisation of nanotubes. 114

Figure 5.2: Schematic diagram for the HNS (Ag-MWCNTs) fabrication. 115

Figure 5.3: Fourier transform infrared spectroscopy (FT-IR) spectrum of p-MWCNTs and f-MWCNTs.

Figure 5.4: Zeta potential measurements as a function of $\mathrm{pH}$, for; raw, carboxylated nanotubes, and nanocomposites. 118

Figure 5.5: Zeta potential measurements as a function of $\mathrm{pH}$ for; raw, carboxylated and silver decorated nanotubes. 119

Figure 5.6: XRD patterns of (A) p-MWCNTs, (B) f-MWCNTs (C) Ag-CNTs, (D) $\mathrm{Fe}_{2} \mathrm{O}_{3}-\mathrm{CNTs}$, (E) $\mathrm{Al}_{2} \mathrm{O}_{3}-\mathrm{CNTs}$, (F) $\mathrm{TiO}_{2}-\mathrm{CNTs},(\mathrm{G}) 10 \mathrm{HNS}$, and (H) $20 \mathrm{HNS}$

Figure 5.7: $5 \mu \mathrm{m}$ (Left) and $500 \mathrm{~nm}$ (Right) SEM images for (A) p-MWCNTs, (B) fMWCNTs

Figure 5.8: $\mu \mathrm{m}$ (Left) and $500 \mathrm{~nm}$ (Right) SEM images for (A) $\mathrm{Fe}_{2} \mathrm{O}_{3}-\mathrm{CNTs}$, (B) $\mathrm{TiO}_{2}-$ CNTs, (C) $\mathrm{Al}_{2} \mathrm{O}_{3}-\mathrm{CNTs}$, and (D) Ag-CNTs

Figure 5.9: $5 \mu \mathrm{m}$ (Left) and $500 \mathrm{~nm}$ (Right) SEM images for (A) 10HNS and (B) 20HNS modulated by microwave irradiation.

Figure 5.10: $2 \mathrm{~mm}, 50 \mu \mathrm{m}$ and $10 \mu \mathrm{m}$ SEM images for $10 \mathrm{HNS}$ synthesised under different microwave irradiation time. 126

Figure 5.11: EDX analysis for (A) p-MWCNTs, (B) f-MWCNTs, (C) $\mathrm{Fe}_{2} \mathrm{O}_{3}$-CNTs, (D) $\mathrm{TiO}_{2}-\mathrm{CNTs}$, (E) $\mathrm{Al}_{2} \mathrm{O}_{3}-\mathrm{CNTs}$ and (F) Ag-CNTs. (G) 10HNS, and (H) 20HNS 127 
Figure 5.12: Thermogravimetric curves of p-MWCNT, f-MWCNTs, 10HNS and 20HNS

Figure 5.13: TGA curves in air for $\mathrm{M} / \mathrm{MO}$ nanocomposites

Figure 5.14: Raman spectroscopy results for pristine, functionalized and metal/metal oxides decorated MWCNTs, $\left(1000 \mathrm{~cm}^{-1}-3000 \mathrm{~cm}^{-1}\right)$. 131

Figure 5.15: Raman spectroscopy results for pristine, functionalized and metal/metal oxides decorated MWCNTs, $\left(100 \mathrm{~cm}^{-1}-1000 \mathrm{~cm}-1\right)$. 132

Figure 5.16: Raman spectroscopy results for pristine, functionalized 10HNS and 20HNS, (A) 1000-3000 $\mathrm{cm}^{-1}$, and (B) 1500-1700 $\mathrm{cm}^{-1}$. 133

Figure 5.17: Full XPS spectra, C 1s and O 1s peak deconvolution of as-received CNTs (A, B and C), and $\mathrm{HNO}_{3}$-treated $\mathrm{MWCNT}(\mathrm{D}, \mathrm{E}$, and F) samples. 135

Figure 5.18: Full XPS spectra (A), Fe 2p (B), C 1s (C) and O 1s (D) peak deconvolution of $\mathrm{Fe}_{2} \mathrm{O}_{3} \mathrm{CNTs}$. 136

Figure 5.19: Full XPS spectra (A), Ti 2p (B), C 1s (C) and O 1s (D) peak deconvolution of $\mathrm{TiO}_{2} \mathrm{CNTs}$.

Figure 5.20: Full XPS spectra (A), Al 2p (B), C 1s (C) and O 1s (D) peak deconvolution of $\mathrm{Al}_{2} \mathrm{O}_{3} \mathrm{CNTs}$. 138

Figure 5.21: Full XPS spectra of AgCNTs (A), 10HNS (B), 20HNS (C), Ag 3d (D), C 1s (E) and O 1s (F) peak deconvolution.

Figure 6.1: The top and bottom surface of (A) nanocomposites prepared with 10HNS and (B) nanocomposites prepared with 20HNS. 146

Figure 6.2: SEM cross section images for (A) Nascent PES membrane, (B) 0.05-10 M, (C) 0.1-10 M, (D) 0.3-10 M, (E) 0.9-10 M, and (F) 0.9-20 M.

Figure 6.3: Contact angle measurements of nanocomposite membranes prepared with different loading weights of $10 \mathrm{HNS}$ and $20 \mathrm{HNS}$. 148

Figure 6.4: Pore size cumulative curves of nanocomposite membranes for 20HNS. 150 Figure 6.5: Surface Zeta potential of nascent and nanocomposites membranes at different loading weight of $10 \mathrm{HNS}$. 
Figure 6.6: Pure water flux behaviour of nanocomposite membranes prepared with $10 \mathrm{HNS}$ and $20 \mathrm{HNS}$ at different loading wt.\%. 153

Figure 6.7: Inhibition zone against EC (Left) and SA (Right) for (A) f-MWCNTs, (B) $10 \mathrm{HNS}$ and (C) 20HNS.

Figure 6.8: Example illustrates the growing colonies of viable S. aureus and E. coli colonies on nutrient agar medium for (A and $\mathrm{C}$ ) control membrane, (B and D) 0.910HNS, and (E) 0.9-20HNS. 157

Figure 6.9: Bactericidal activities of nanocomposites against E. coli and S. aureus. 158 Figure 6.10: Variation in the antibacterial activity against E.coli for nanocomposites prepared using $10 \mathrm{HNS}$ and $20 \mathrm{HNS}$ at 0.9 loading $\mathrm{wt} \%$ to the polymer. 159

Figure 6.11: $5 \mu \mathrm{m}$ and $20 \mu \mathrm{m}$ SEM micrographs of (A) virgin control PES membrane, (B) virgin 0.9-10M nanocomposite, (C) Virgin 0.9-20M nanocomposite 160

Figure 6.12: Surface SEM image for AgNPs/PES nanocomposite membrane. $3 \mu \mathrm{m}$ (Left) and $40 \mu \mathrm{m}$ (Right)

Figure 6.13: (A) control PES membrane fouled with S. aureus, (B) 0.9-10M nanocomposite fouled with S. aureus, (C) control PES membrane fouled with E. coli and (D) 0.9-10M nanocomposite fouled with E. coli. 162

Figure 7.1: Photographic view of $\mathrm{Fe}_{2} \mathrm{O}_{3}-\mathrm{CNTs}$ nanocomposites: (A) dispersed in water, (B) their response to a magnetic field.

Figure 7.2: TFN membrane fabrication, acrylic frame and vacuum unit (left), and TFN membrane sheet (right).

Figure 7.3: Fourier transform infrared spectroscopy (FT-IR) spectrum of r-CNTs, fCNTs, f-CNTs-PDA and PDA coated nanocomposites. 170

Figure 7.4: TGA curves in the air for PDA coated nanocomposites 171

Figure 7.5: Zeta potential measurements as a function of $\mathrm{pH}$, for PDA coated nanocomposites 172

Figure 7.6: FT-IR spectra of the synthesised PES, TFC and TFN membranes 173

Figure 7.7: SEM surface images for; (A) TFC, (B) f-CNTs-TFN, (C) Ag-TFN, (D) $\mathrm{Al}_{2} \mathrm{O}_{3}$-TFN, (E) $\mathrm{Fe}_{2} \mathrm{O}_{3}-\mathrm{TFN},(\mathrm{F}) \mathrm{TiO}_{2}$-TFN 
Figure 7.8: SEM cross-section images for; (A) TFC, (B) f-CNTs-TFN, (C) Ag-TFN, (D) $\mathrm{Al}_{2} \mathrm{O}_{3}$-TFN, (E) $\mathrm{Fe}_{2} \mathrm{O}_{3}$-TFN, (F) $\mathrm{TiO}_{2}$-TFN. 175

Figure 7.9: Three-dimensional-AFM images $(5 \mu \mathrm{m} \times 5 \mu \mathrm{m})$ of; (A) TFC, (B) f-CNTTFN, (C) $\mathrm{Al}_{2} \mathrm{O}_{3}-\mathrm{TFN},(\mathrm{D}) \mathrm{Fe}_{2} \mathrm{O}_{3}-\mathrm{TFN},(\mathrm{E}) \mathrm{TiO}_{2}-\mathrm{TFN}$ and (F) Ag-TFN.

Figure 7.10: Static contact angle measurements of TFC and TFN membranes 178

Figure 7.11: Pure water and solute permeation of NF membranes against; (A) NaCl, (B) $\mathrm{Na}_{2} \mathrm{SO}_{4},(\mathrm{C}) \mathrm{MgSO}_{4}$ and (D) ternary salt solution. (2000 ppm, $\left.21{ }^{\circ} \mathrm{C}, 1 \mathrm{MPa}\right) . .180$

Figure 7.12: Pure water and solute rejection of NF membranes against; (A) NaCl, (B) $\mathrm{Na}_{2} \mathrm{SO}_{4},(\mathrm{C}) \mathrm{MgSO}_{4}$ and (D) ternary salt solution. $\left(2000 \mathrm{ppm}, 25^{\circ} \mathrm{C}, 1 \mathrm{MPa}\right) \ldots \ldots . .181$

Figure 7.13: Peeling off the PA-HNS thin film layer under cross-flow conditions (Left), and TFN membrane prepared in this work (Right). 182

Figure 7.14: Calibration curve of heavy metal solutions determined by AAS, (A) Nickel, (B) Manganese, (C) Copper, (D) Cobalt, and (E) Lead. 184

Figure 7.15: PWF vs solute flux of metal mixture (A), retention vs PWF for (B) $\mathrm{Mn}^{2+}$, (C) $\mathrm{Ni}^{2+}$, (D) $\mathrm{Pb}^{2+}$, (E) $\mathrm{Cu}^{2+}$, and (F) $\mathrm{Co}^{2+}$. (feed concentration= $250 \mathrm{ppm}, 21^{\circ} \mathrm{C}, \mathrm{pH}=5$, $1 \mathrm{MPa})$. 185 


\section{Tables}

Table 1.1: Commonly used polymers and membrane fabrication techniques in water treatment processes (Hilal et al., 2015).

Table 2.1: Properties of graphene, CNT, nano-sized steel and polymers (Kuilla et al., 2010).

Table 2.2: Influence of various types of ENMs on polymeric membranes

Table 3.1: Specification of the MWCNTs

Table 4.1: Composition of PES membranes .86

Table 4.2: Surface roughness parameters of the membranes. .88

Table 4.3: Geometric mean pore size, standard deviation and MWCO of membranes.

Table 4.4: Mechanical characteristics of UF membranes. 94

Table 4.5: Total, reversible and irreversible fouling data of membranes, as a function of initial feed solution $\mathrm{pH}$ 108

Table 4.6: Total, reversible and irreversible fouling data of membranes, as a function of initial feed concentration.

Table 4.7: Summary for PES wt $\%$ influence on characteristics of membranes. 110

Table 5.1: Composition and decomposition technique used during the nanocomposite material fabrication. 116

Table 6.1: Compositions of nanocomposite membranes using two concentrations of silver to nanotubes. 145

Table 6.2: Geometric mean pore size and standard deviation of nanocomposite membranes.

Table 6.3: Pore size cumulative curves of nanocomposite membranes for 10HNS. .150

Table 7.1: Average surface roughness parameters of TFC and TFN membranes .....176 


\section{Nomenclatures}

$\mu \mathrm{m}$

a

$\mathrm{Ag}$

$\mathrm{Ag}^{+}$

$\mathrm{AgNO}_{3}$

Ag-NPs

$\mathrm{Al}\left(\mathrm{NO}_{3}\right)_{3} .9 \mathrm{H}_{2} \mathrm{O}$

$\mathrm{Al}_{2} \mathrm{O}_{3}$

bio $\mathrm{Ag}^{0}$

$\mathrm{C}_{\mathrm{f}}$

$\mathrm{C}_{\mathrm{p}}$

$\mathrm{Cu}$

$\mathrm{Cu}_{2} \mathrm{O}$

$\mathrm{CuO}-\mathrm{NPs}$

$\mathrm{D}_{\mathrm{AB}}$

$d_{\mathrm{p}}$

$d \mathrm{~s}$
Micrometre

Stokes radius $(\mathrm{m})$

Silver

Silver ions

Silver nitrate

Silver nanoparticles

Aluminium nitrate nonahydrate

Aluminium oxide

Biogenic silver nanoparticles

Concentration in the feed solutions

Concentration in the permeate solutions

Copper

Copper oxide

Copper oxide nanoparticles

diffusivity $\left(\mathrm{m}^{2} / \mathrm{s}\right)$

Diameter of pore

Solute diameter 


\begin{tabular}{|c|c|}
\hline $\mathrm{Fe}^{+2}$ & Iron(II) \\
\hline $\mathrm{Fe}\left(\mathrm{NO}_{3}\right)_{3} \cdot 9 \mathrm{H}_{2} \mathrm{O}$ & Iron(III) nitrate nonahydrate \\
\hline $\mathrm{Fe}^{+3}$ & Iron(III) \\
\hline $\mathrm{Fe}_{2} \mathrm{O}_{3}$ & Iron oxide \\
\hline $\mathrm{Fe}_{3} \mathrm{O}_{4}$ & Magnetite \\
\hline $\mathrm{Fe}-\mathrm{NPs}$ & Iron nanoparticles \\
\hline $\mathrm{f}-\mathrm{GO}$ & Functionalized Graphene Oxide \\
\hline$F_{\text {ir }}$ & Irreversible fouling \\
\hline$F_{\text {rev }}$ & Reversible fouling \\
\hline $\mathrm{F}_{\mathrm{T}}$ & Total fouling \\
\hline $\mathrm{G}$ & Graphene \\
\hline GO & Graphene oxide \\
\hline $\mathrm{H}_{2} \mathrm{SO}_{4}$ & Sulphuric acid \\
\hline $\mathrm{HNO}_{3}$ & Nitric acid \\
\hline $\mathrm{J}_{1}$ & Solute flux \\
\hline $\mathrm{J}_{2}$ & Water flux of fouled membrane after water flushing \\
\hline $\mathrm{J}_{\mathrm{o}}$ & Initial water flux of virgin membranes \\
\hline $\mathrm{k}$ & Boltzmann's constant \\
\hline $\mathrm{kDa}$ & Kilo Dalton \\
\hline$M$ & molecular weight \\
\hline $\mathrm{MgSO}_{4}$ & Magnesium sulfate \\
\hline
\end{tabular}




\begin{tabular}{|c|c|}
\hline $\mathrm{mM}$ & Millimolar \\
\hline $\mathrm{mM}$ & Mili-molar \\
\hline $\mathrm{MPa}$ & Megapascal \\
\hline $\mathrm{mV}$ & Millivolt \\
\hline $\mathrm{Na}_{2} \mathrm{SO}_{4}$ & Sodium sulfate \\
\hline $\mathrm{NaCl}$ & Sodium chloride \\
\hline $\mathrm{nSe}$ & Selenium nanoparticles \\
\hline $\mathrm{R} \%$ & Rejection percent \\
\hline $\mathrm{R}^{2}$ & Regression correlation \\
\hline $\mathrm{R}_{\mathrm{a}}$ & Root average arithmetic roughness \\
\hline $\mathrm{R}_{\max }$ & Ridge to valley distance \\
\hline $\mathrm{R}_{\mathrm{ms}}$ & Root mean square roughness \\
\hline $\mathrm{SiO}_{2}$ & Silicon oxide \\
\hline $\mathrm{Ti}\left(\mathrm{OCH}_{2} \mathrm{CH}_{2} \mathrm{CH}_{2} \mathrm{CH}_{3}\right)_{4}$ & Titanium (IV) butoxide. \\
\hline $\mathrm{TiO}_{2}$ & Titanium dioxide \\
\hline $\mathrm{TiO}_{2}$-NPs & Titanium dioxide nanoparticles \\
\hline $\mathrm{ZnO}$ & Zinc oxide \\
\hline $\mathrm{ZrO}_{2}$ & Zirconium oxide \\
\hline$\alpha-\mathrm{Fe}_{2} \mathrm{O}_{3}$ & Hematite \\
\hline$\gamma-\mathrm{Fe}_{2} \mathrm{O}_{3}$ & Maghemite \\
\hline
\end{tabular}




\section{Greek letters}

$\begin{array}{ll}{[\eta]} & \text { Intrinsic viscosity } \\ \mu_{\mathrm{p}} & \text { Mean pore size } \\ \eta & \text { Solvent viscosity } \\ \zeta & \text { Zeta potential } \\ \sigma_{\mathrm{p}} & \text { Geometric standard deviation }\end{array}$

\section{Abbreviations}

0D

$10 \mathrm{HNS}$

$1 \mathrm{D}$

20HNS

2D

3D

AAPTS

AFM

APTES

APTS

$\mathrm{BE}$

$\mathrm{BNC}$
Zero Dimension

Hybrid nanostructure prepared with $10 \mathrm{wt} \%$ silver

One Dimension

Hybrid nanostructure prepared with $20 \mathrm{wt} \%$ silver

Two Dimension

Three Dimension

N- [3- (trimethoxysilyl)propyl] ethylenediamine

Atomic force microscopy

Aminopropyltriethoxysilane

3-aminopropyltriethoxysilane

Binding energy

Bacterial nanocellulose 
BSA

CA

CFU

$\mathrm{CNC}$

CNTs

CVD

DA

DDA

DI

DNA

DWCNTs

EC

EDX

ENMs

FE-SEM

f-MWCNTs

FTIR

HA

$\mathrm{HCl}$

HNS

HPEI
Bovine Serum Albumin

Contact angles

Colony forming unit

Cellulose Nanocrystal

Carbone nanotubes

Chemical vapour deposition

Dopamine

Dodecyl amine

De-ionized water

Deoxyribonucleic acid

Double-Walled Carbon Nanotubes

E. coli

X-ray energy dispersive spectroscopy

Engineered Nanoscale Materials

Field Emission Scanning Electron Microscopy

Functionalized multiwalled carbon nanotubes

Fourier transmission infrared

Humic acid

Hydrochloric acid

Hybrid nanostructure

Hyperbranched polyethyleneimine 
IEP

LBL

LDE

MBR

MF

MHB

MMMs

MPD

MWCNTs

MWCO

$\mathrm{NaAlg}$

$\mathrm{NaX}$

$\mathrm{NC}$

NF

NFC

NMP

NMs

NOM

n-TFN

NWs

nZVI
Isoelectric point

Layer By Layer

Laser doppler electrophoreses

Membrane bioreactor

Microfiltration

Mueller Hinton Broth

Mixed matrix membranes

Phenylenediamine

Multi-walled carbon nanotubes

Molecular weight cut-off

Sodium alginate

Zeolite

Nanocomposite

Nanofiltration

nano-fibrillated cellulose

N-methyl-2-pyrrolidone

Nanoscale materials

Natural organic matters

Thin-Film Nanocomposite

Nanowires

Zero-Valent Iron nanoparticles 
OVA

PA

PBS

PDA

PEG

PEO

PES

PI

PMMA

p-MWNT

PS

PSD

PSF

PVA

PVDF

PVP

PWF

$\mathrm{RF}$

RF

RH

RH
Ovalbumin

Polyamide

Phosphate Buffer Solution

Polydopamine

Polyethylene glycol

Polyethylene oxide

Polyethersulfone

phase inversion

Poly (methyl methacrylate)

Pristine multiwalled carbon nanotubes

phase separation

Pore size distribution

Polysulfone

Polyvinyl alcohol

Polyvinylidene fluoride

Polyvinylpyrrolidone

Pure water flux

Relative flux

Relative flux

Relative humidity

Relative humidity 
RO

ROS

SA

SEM

SMA

SWCNTs

TEM

TFC

TFN

TGA

TMC

UF

UV

$\mathrm{w} / \mathrm{v}$

WCA

XPS

XRD

ZOI

ZP
Reverse osmosis

Generate reactive oxygen species

S. aureus

Scanning electron microscopy

Styrene-alt-maleic anhydride

Single-walled carbon nanotubes

Transmission Electron Microscopy

Thin film composite

Thin film nanocomposite

Thermal gravimetric analysis

1,3,5-Benzenetricarbonyl trichloride

Ultrafiltration

Ultraviolet

Weight/volume ratio

Water contact angle

X-ray photoelectron spectroscopy

X-ray diffraction

Zone of inhibition

Zeta potential 


\section{Chapter One}

\section{Introduction}

\subsection{General Introduction}

There is currently a wealth of research activity developing novel nanocomposite materials that harness the benefits of engineered nanoscale materials (ENMs). Indeed, one of the legacies of nanotechnology has been improved design and control of nanoparticles and ultimately nanocomposite materials. This has gone hand in hand with improvements in technologies, such as atomic force microscopy (AFM), that enable characterisation of materials at the nanoscale and so optimisation of the nanoscale materials as they are developed. Thus, many tools and processes are now available to optimise the engineering of nanocomposite materials. This offers great potential for the fabrication of novel membranes for desalination and water treatment, and this research showcases the flourishing research community that has been established and is now meeting the opportunities and challenges presented by ENMs.

Much effort in the last decade has been focused on fabricating synthetic membranes for particular applications with desired characteristics such as selectivity, permeability, structure, chemical and physical properties. To achieve this goal, several techniques have been implemented such as phase inversion, stretching, track-etching, sintering, interfacial polymerisation and electrospinning (Lalia et al., 2013). Membranes used in water treatment applications can be made from a wide variety of inorganic and organic materials; inorganic material includes ceramics, metals, and glass; organic materials include polymers, composite materials or mixed matrixes (Ulbricht, 2006). Inorganic membrane fabrication has recently gained attention due to their high mechanical strength and chemical resistance; however, their applicability for water treatment purposes is restricted due to the high fabrication costs and preparation 
difficulties ( $\mathrm{Ng}$ et al., 2013). In contrast, polymeric membranes are much preferable in industrial applications. Their selectivity, variety of membrane structures and properties, ease of preparation and pore formation control and the inexpensiveness of polymers have meant that they dominate in membrane applications (Hilal et al., 2015). Some of these polymers are listed in Table 1.1.

Table 1.1: Commonly used polymers and membrane fabrication techniques in water treatment processes (Hilal et al., 2015).

\begin{tabular}{|c|c|c|}
\hline $\begin{array}{l}\text { Water treatment } \\
\text { processes }\end{array}$ & $\begin{array}{l}\text { Polymers used for membranes } \\
\text { fabrication }\end{array}$ & Fabrication techniques \\
\hline Reverse osmosis & $\begin{array}{l}\text { Cellulose acetate/triacetate } \\
\text { Aromatic polyamide } \\
\text { Polypiperazine } \\
\text { Polybenzimidazoline }\end{array}$ & $\begin{array}{l}\text { Phase inversion } \\
\text { Interfacial polymerization }\end{array}$ \\
\hline Nanofiltration & $\begin{array}{l}\text { Polyamide } \\
\text { Polysulfone } \\
\text { Polyol } \\
\text { Polyphenol }\end{array}$ & $\begin{array}{l}\text { Interfacial polymerization } \\
\text { Phase inversion }\end{array}$ \\
\hline Ultrafiltration & $\begin{array}{l}\text { Polyacrylonitrile } \\
\text { Polyethersulfone } \\
\text { Polysulfone } \\
\text { Poly(phthalazineone ether sulfone } \\
\text { ketone) } \\
\text { Poly(vinyl butyral) } \\
\text { Polyvinylidene fluoride }\end{array}$ & Phase inversion \\
\hline Microfiltration & $\begin{array}{l}\text { Polyvinylidene fluoride } \\
\text { Poly(tetrafluoroethylene) } \\
\text { Polypropylene } \\
\text { Polyethylene } \\
\text { Polyethersulfone } \\
\text { Polyetheretherketone }\end{array}$ & $\begin{array}{l}\text { Phase inversion } \\
\text { Stretching } \\
\text { Track-etching }\end{array}$ \\
\hline Membrane distillation & $\begin{array}{l}\text { Poly(tetrafluorethylene) } \\
\text { Polyvinylidene fluoride }\end{array}$ & $\begin{array}{l}\text { Phase inversion } \\
\text { Stretching } \\
\text { Electrospinning }\end{array}$ \\
\hline
\end{tabular}

Despite the relatively advanced state of the membrane industry, there are still some issues that need to be tackled for large-scale applications. Fouling is among the main bottlenecks of the membrane technology to compete with conventional water treatment technologies (Nackaerts, 2014). Membrane fouling occurs due to the accumulation of various solutes on the membrane surface and interior structure of the membrane, forming an additional barrier at the membrane surface or blocking the internal pores. 
This hinders the solvent from passing through the membrane, reducing permeation and raising the transmembrane pressure required to maintain the same productivity. Thus, ultimately shortening the membrane's lifespan. Moreover, fouled membranes may consume a massive amount of cleaning chemicals, which may also impact on the membrane surface and lead to membrane replacement in severe cases. The consequence of all these issues is to increase the operation and the maintenance costs of the water treatment unit (Boussu et al., 2007; Contreras, 2011; Hilal et al., 2005). The good selection of membrane materials available, module and design, operating conditions, pretreatment processes and conditions could mitigate the fouling phenomena to some extent; however, membrane sustainability is still problematical at the industrial scale and represents a challenging issue due to its complexity and variety (Hilal et al., 2012; Mohammad et al., 2003). For several decades, membrane fouling phenomena have been widely addressed from many angles in attempts to minimise their consequences, for instance, understanding fouling mechanisms, incidence, types, and factors affecting fouling growth (Becht, 2008).

Membrane modification is a method by which the hydrophilicity of the membrane can be tailored to reduce the fouling from the components of the process fluid. Indeed, some argue that membrane modification can be defined as the process of incorporation of a hydrophilic functional group at the surface of a membrane, aiming to enhance the free surface energy and thereby reducing fouling since the interactions of most foulants with membranes are hydrophobic in nature (Rana \& Matsuura, 2010; Sathish Kumar et $a l ., 2015)$. In fact, membrane separation processes are surface dependent, where the membrane's active layer (skin) controls the separation process and the membranefoulant interactions. Introducing a hydrophilic functional group to that surface is believed to improve the separation performance of the membrane and to reduce/control the undesired adhesion and/or adsorption interactions between foulants and that active layer (Kochkodan et al., 2006; Kochkodan et al., 2014). For achieving this, an assortment of methods has been suggested which could be used individually or in combination (Mohammad et al., 2015; Van der Bruggen, 2009). These surface modifications include grafting (Hua et al., 2008), surface chemical reaction (Wang et al., 2006b), blending (Wang et al., 2006c; Yu et al., 2009; Zhao et al., 2011b), plasma treatment (Yu et al., 2005b), dip coating (Madaeni \& Ghaemi, 2007) and ion implantation (Mukherjee et al., 2005a). A variety of polymeric, organic and inorganic 
compounds and nanoscale materials can be utilised via these techniques to improve polymeric membrane hydrophilicity.

Recently, the incorporation of ENMs into a polymeric membrane matrix has gained significant attention for water and wastewater treatment applications (Wang et al., 2013). The fabrication of nanocomposite membranes that conserve the advantages of polymeric membranes yet overcome their disadvantages by incorporation of ENMs is a highly desired outcome for membrane development. Nanocomposite membranes are a new class of membranes consisting of both organic polymers and inorganic nanoscale materials, which are believed to exhibit enhanced performance in comparison to standard membranes (Kango et al., 2013; Pendergast \& Hoek, 2011; Qu et al., 2013). The membrane that merges the beneficial properties of both organic and inorganic materials to create a new membrane with enhanced hydrophilicity, permeability, thermal and chemical stability, porosity and mechanical properties has been sought by many research groups (Kumar et al., 2009; Zhao et al., 2013). However, many processes and environmentally disruptive issues can arise from incorporation of ENMs into polymeric membranes, such as disruption of membrane morphology and particulate leaching, these will impact on process efficiency. Choosing applicationspecific nanomaterials with an optimum composition is essential to overcome limitations in polymeric membrane applications ( $\mathrm{Ng}$ et al., 2013).

There are a large number of studies that have used different ENMs in the development of novel composite polymer membranes for water treatment applications. The materials that have been studied include, graphene oxide (GO) (Xia \& Ni, 2015; Xu et al., 2014), carbon nanotubes (CNTs) (Celik et al., 2011b; Shen et al., 2013; Vatanpour et al., 2011), silver (Ag) (Kim et al., 2012; Prince et al., 2014), titanium $\left(\mathrm{TiO}_{2}\right)$ (Li et al., 2009; Rahimpour et al., 2008; Shi et al., 2013), aluminum $\left(\mathrm{Al}_{2} \mathrm{O}_{3}\right)$ (María Arsuaga et al., 2013), silicon $\left(\mathrm{SiO}_{2}\right)$ (Yu et al., 2009), iron ( $\left.\mathrm{Fe}_{3} \mathrm{O}_{4}\right)$ (Alam et al., 2013), zirconium $\left(\mathrm{ZrO}_{2}\right)$ (Maximous et al., 2010) and zinc ( $\left.\mathrm{ZnO}\right)$ (Balta et al., 2012), clay nanoparticles (Mierzwa et al., 2013) and zeolite (NaX) (Fathizadeh et al., 2011; Jeong et al., 2007). 


\subsection{Outline of the thesis}

This thesis comprises eight chapters. The outlines of each chapter have been specified as follows: The introductory chapter (Chapter 1) showcases the great potentials opportunities of engineered nanoscale materials (ENMs) in the design and fabrication of engineering nanocomposite membranes for desalination and water treatments along with their challenges. The focus of the literature review (Chapter 2) is mainly on the modification of polymeric membranes using a diverse range of ENMs; this includes the features of ENMs, strategies of incorporation, the influence of ENMs on polymeric membranes surface characteristics and antifouling properties and issues associated from the incorporation of ENMs. The review sheds light on findings have not been covered in previous research. It gives an overview on the wide range of nanoscale materials (metal/metal oxide nanomaterials, carbon-based, and for the first time, cellulose nanoscale materials). In addition to addressing the advantages and main issues associated with incorporating these nanomaterials (environmental and cost issues) and presents the recent attempts to improve the compatibility with polymeric membranes to overcome these issues. All experimental related procedures and chemicals used in this thesis have described in (Chapter 3). In the first results chapter (Chapter 4), an investigation was made into the influence of PES concentration on UF membrane morphology and surface characteristics. PES/UF membranes have synthesised with a wide range of structures that correspond to typical commercially available UF membranes. Fouling behaviour and retention efficiency tests were conducted to give a more precise and comprehensive assessment of UF membrane fouling. Three different organic model foulants (HA, BSA, and $\mathrm{NaAlg}$ ) have been employed for this comprehensive assessment under a broad range of feed concentration and solution $\mathrm{pH}$. In the second results chapter (Chapter 5), versatile metal/metal oxides-carbon nanotubes, as hybrid nanostructures, have been synthesised via facile, scalable and costeffective routes. This was carried via direct precipitation and decomposing of silver, iron, titanium and aluminium metal salt solutions on the surface of nanotubes with the aid of either microwave irradiation or high-temperature tube furnace along with comprehensive characterisation for the nanostructures. In (Chapter 6), we aim to shed the light on developing a nanocomposite polymeric membrane using Ag-CNTs based HNS as antibacterial additives. The bacteriostatic activity of the prepared silver-based HNS tested as a powder. After that, impregnated within the polymeric matrix and tested 
again for evaluating the antibacterial properties of the nanocomposite membranes against two pathogens. The last chapter of results (Chapter 7) discussed the application of prepared HNS in desalination. Four different M/MO-MWCNTs based HNS were coated with a PDA layer to get the final product. Next, a thin layer of HNS deposited on a porous substrate membrane by vacuum filtration and the PA layer was adjusted to entirely cover and robustly fix the HNS interlayer in order to produce a defect-free TFN membrane. The performance of produced thin film nanocomposite evaluated against monovalent, divalent salts and heavy metals solution. Finally, the comprehensive conclusion remarks, recommendations, and future works are presented in (Chapter 8). 


\section{Chapter Two}

\section{Literature Review}

This chapter prefaces three major themes and commences with an overview of membrane separation processes; including their potentialities, applications, and classifications. Also, it discusses various materials and techniques employed for membranes synthesis. Second, covers the inherent impediment associated with membranes separation, or what so-called "membranes fouling", as well as various foulants and factors influence the fouling extent in the membranes. Finally, the modification techniques employed to diminish membrane fouling are reviewed, with a focus made on polymeric membranes modification that harnesses ENMs. This will include ENM's classification, incorporation strategies and how their properties impact on the surface characteristics, robustness, functionality, morphologies and antifouling properties of polymeric membranes. The review will also feature a discussion on the current impediments facing the development and commercialisation of nanocomposite membrane that harnesses the benefits of ENMs.

\subsection{An overview of membrane separation processes}

The demand for membrane technology for surface water, wastewaters and desalination applications continue to grow in recent years. Membrane separation is one of the potent separation tools in many industrial applications like; wastewater treatment, drinking water production, electronics, medical treatment, paper and pulp, fuel cells, pharmaceutical, biotechnology, and food industries (Zeman \& Zydney, 1996). Simply, a membrane defined as a selective barrier that allows one or more species to transfer from a bulk phase to another one separated by the membrane while prevents other species from the passage (Ulbricht, 2006). Taking into the account its cost and energy efficiency, if compared with other conventional separation technologies, introducing 
the membrane technology encouraged these industrial applications to become ecofriendlier by facilitating the recycling of waste materials and resource recovery that may contribute to waste production (Rohani et al., 2011). Besides that, they exhibit high potentialities in the rationalisation of production systems (Strathmann et al., 2011). Industrial operations exploit membranes technology do not require many additives, downscaling and upscaling of membranes, and can easily integrated into other separation techniques. Also, they operate isothermally at low temperature and utilise lower energy consumption in comparison to the other conventional separation processes (Ulbricht, 2006).

Classification of membranes can base on different criteria. According to Aptel \& Buckley (1996), the mass transfer through membranes could take place based on several types of driving forces (i.e. pressure, concentration gradient, temperature and electrical potential difference). A pressure difference is defined as the driven force for MF, UF, $\mathrm{NF}$ and RO membranes in water treatment applications. Mass transfer in liquid phase pressure-driven membrane processes is a function of the complex microhydrodynamics and interfacial events occurring at or inside the membrane surface. The movement of a material or solute in a membrane process transitions through a series of different mass transfer regions, namely movement from the bulk solution to the membrane surface via a concentration polarisation layer, equilibrium partitioning from the bulk solution to a space just inside the membrane pore, travel through the membrane pore itself and then equilibrium partitioning from just inside the exit of the pore to the bulk permeate. Each of these individual processes occurs in series and a change in anyone of the processes has a knock-on effect on the subsequent processes.

Commonly, pressure-driven membrane processes are categorised into four broad types depending on the pore size of the membrane, includes; microfiltration (MF), ultrafiltration (UF), nanofiltration (NF) and reverse osmosis (RO) membranes, in addition to other criteria (Baker, 2004). MF membranes, with a pore size range (0.05$10 \mu \mathrm{m}$ ), employed extensively across a wide range of industries for various applications that usually involve the removal of suspended solids, colloids, and macromolecules by the sieving mechanism. MF can possess a water permeability higher than $500 \mathrm{LMH}$ and operates under less than 2 bars. Instead of conventional filters, MF has been efficiently used in desalination and food industry as a pre-treatment process (Baker, 2004; Hilal et $a l ., 2015)$. UF membranes, with a pore size range $(5-100 \mathrm{~nm})$, is another attractive 
separation process used for micro solute separation, and utilise an operating pressure of fewer than 5 bars. It is well known as a clarification and disinfection separation process having a wide range of applications, includes wastewater treatment, fractionating of proteins and drinking water disinfection (Baker, 2004). Typically, filtrate flux rate of UF membranes ranging from 25 to $250 \mathrm{LMH}$ (Desai, 2000). NF membrane is another superior separation process. It is also called membrane softening or low-pressure RO since it falls between UF and RO in term of its selectivity and operating pressure, as shown in Figure 2.1. With approximately $1 \mathrm{~nm}$ pore size, solute transport through NF membranes is stemmed by convection as a result of applied pressure difference and by diffusion as a result of concentration gradient differences across membranes (Otero et $a l ., 2008)$. NF used to adjust the ratio of monovalent salts, separate multivalent salts and dissolved organic solutes depending on size exclusion and dielectric effects. NF membranes are of special interest due to their higher flux rates and lower energy consumption. Therefore, introduced to replace RO membranes in many applications includes; drinking water, ultra-pure water production, and dyes removal (Raman et al., 1994; Watson \& Hornburg, 1989). Currently, about 65\% of the NF market is accounted for by water treatment applications, $25 \%$ of the market for the food and dairy sectors and less than $10 \%$ for the chemical industries. Finally, reverse osmosis (RO) membrane with the densest structure among other membrane processes. RO represent about $50 \%$ in desalination plants of brackish and seawater while other applications include pharmaceutical, power generation industry and small application in food and pollution control. With less than $1 \mathrm{~nm}$ pore size, solution diffusion is the main transport mechanism through RO membranes that require a high pressure (10-100 bar) to perform the separation through the membrane dense layer (Baker, 2004). 


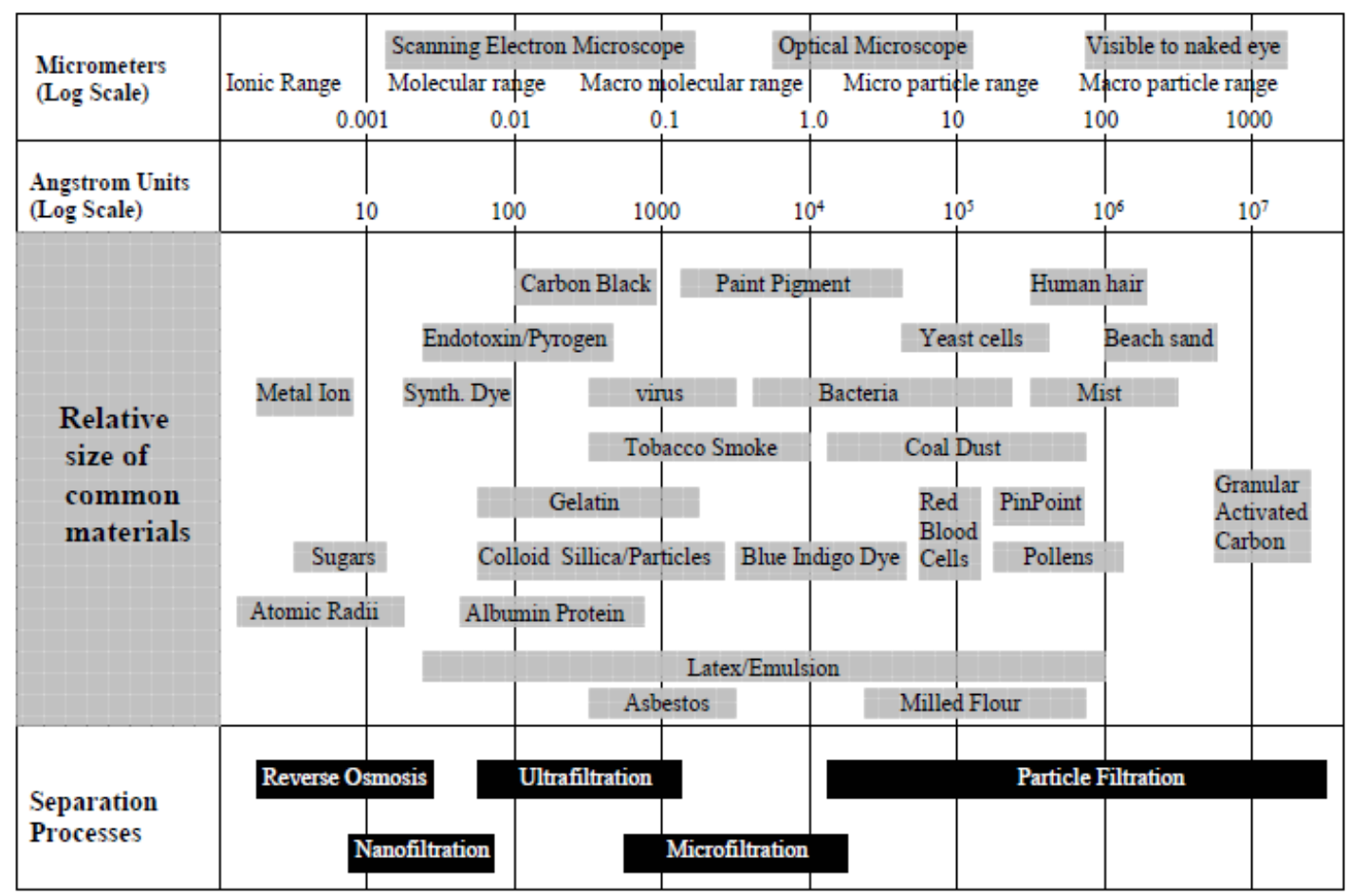

Figure 2.1: Classification of membrane separation processes, (Donnelly et al., 1998).

From the other hand, membranes can also be classified in terms of their structure, preparation techniques, geometry, and fabrication materials. These classifications give an indication of the membrane specifications (Baker, 2004). Membrane's structure can be divided into broad types; e.g., thin layer, bi or multilayer, mixed matrix composite, symmetric (isotropic) with uniform structure and composition, and asymmetric (anisotropic) consists of a thin dense layer which performs the separation process supported on a much thicker substrate to provide the required mechanical strength, as shown in Figure 2.2. However, classification based on the fabrication techniques includes well-known examples of Loeb- Sourirajan (polymer precipitation process), in situ polymerisation, sol-gel process, sintering, stretching, track-etching and solution coated composite membrane. Also, geometry classification refers to flat sheets and hollow fibres produced by the various techniques. These membranes are packaged into efficient and economic modules with the high surface area to increase membrane flux and reduce fouling (Baker, 2004). Membranes can also be classified into organic, inorganic and mixed matrix composite membranes based on their fabrication materials (Dang, 2009). 


\section{Symmetrical membranes}
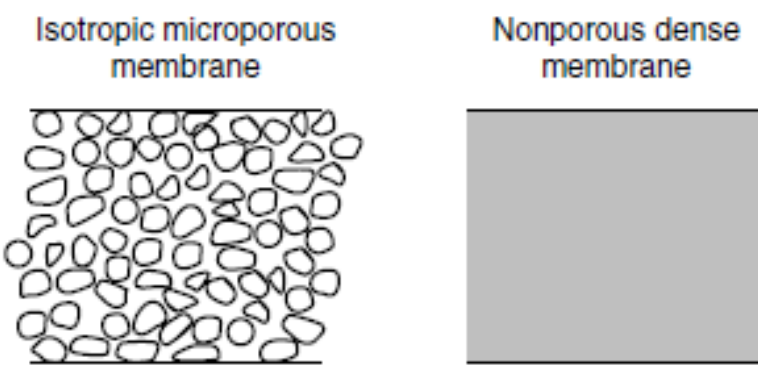

Anisptropic membranes

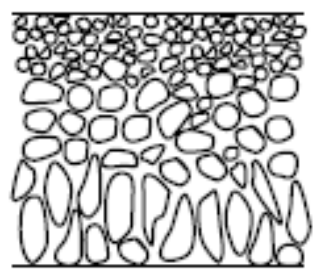

Loeb-Sourirajan anisotropic membrane

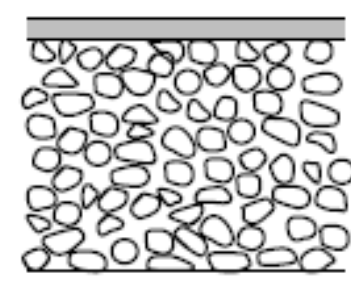

Thin-film composite anisotropic membrane
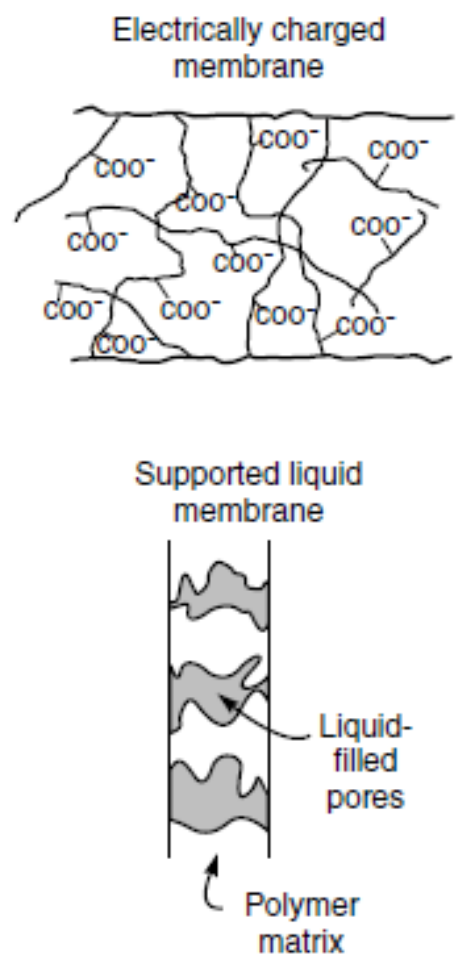

Figure 2.2: Schematic diagrams of the principal types of membrane(Baker, 2004)

\subsection{Membranes fabrication}

Worthwhile efforts have been devoted in the last decades to fabricate synthetic membranes for a particular application with a desired selectivity, permeability, structure, chemical and physical properties. To achieve this goal, several techniques were implemented depending on the desired specifications and applications; this includes phase inversion, stretching, track-etching, sintering, interfacial polymerisation and electrospinning (Lalia et al., 2013). Since the focus of this thesis is onto flat-sheet membranes, the phase inversion (PI) method will be highlighted from different angles including; PI types, mechanism, fabrication materials and some of the critical factors that can influence the fabricated membrane properties.

\subsubsection{Membrane materials}

Membranes used in water treatment applications are made from a wide variety of materials. Inorganic material (Ceramic, metal, glass, and liquid), organic polymers, composite materials or mixed matrixes are the standard materials used in membranes 
preparation (Ulbricht, 2006). However, the emerging industrial membranes marketplace of inorganic materials is still in its initial stages if compared to the available organic polymers employed for membranes fabrication.

Although inorganic membranes fabrication has recently received much attraction due to their high mechanical strength and chemical resistance, their applicability for water treatment purposes is limited, due to many issues associated with the fabrication process, such as high fabrication costs and preparation difficulties. For instance, pore size distribution control and relatively low flux are the main obstacles in ceramic membranes preparation process ( $\mathrm{Ng}$ et al., 2013). In contrast, polymeric membranes are much preferable in industrial applications. Their selectivity, variety of membrane structures and properties, ease of preparation and pore formation control and inexpensiveness of polymers stand behind their domination.

In the organic membranes fabrication, a polymer should be chosen qualitatively, based on particular specifications originated from structural factors (Mulder, 1996). The polymer chosen for a successful membrane formation must: (i) be compatible with the intended application, (ii) be a suitable membrane former, (iii) be reasonably priced, available and applicable to the membrane fabrication technique (Zeman \& Zydney, 1996). According to Mulder (1996), several polymers exploited for membranes fabrication, this includes;

i. Cellulose derivatives; Cellulose Nitrate and Cellulose Acetate are highly crystalline and hydrophilic polymers. Although membranes made from cellulosebased polymers exhibit low fouling tendency and ease of membrane flux restoration; their thermal, chemical and biological resistance is relatively low.

ii. Polyamides (PA); an aromatic polyamide and aliphatic polyamide have excellent thermal, chemical and hydraulic stability. Aromatic polyamides are widely used for RO membrane fabrication while aliphatic polyamides used for UF and MF membranes. However, polyamides have low compatibility with other polymers and solvents.

iii. Polyethylene derivatives; polytetrafluoroethylene (PTFE), poly (vinylidenefluoroethylene) (PVDF) and polypropylene are highly crystalline and hydrophobic polymers. Also, they have excellent thermal and chemical stability. 
iv. Polycarbonates have excellent thermal and mechanical characteristics, and widely used for MF membrane fabrication.

v. Sulfone based polymers; polyethersulfone and polysulfone (PS) are quite essential polymers, not only because they are used for UF membrane fabrication but also as substrates for NF and RO membrane processes. Also, they exhibit a good thermal and chemical stability. Even though that polysulfone is less hydrophilic than polyethersulfone, PS is still preferable when mechanical and thermal characteristics are extensively required. PES has been widely used as the host polymer in membranes modification due to its simple main chain. Moreover, PES has high chlorine resistance within a short contact time, afford a wide range of $\mathrm{pH}(3-13)$ and temperature (up to $75^{\circ} \mathrm{C}$ ). However, the hydrophobicity of PES is still the main obstacle of this polymer.

\subsubsection{Phase inversion}

Since the invention of phase inversion (PI) or phase separation (PS) technique pioneered by (Loeb \& Sourirajan, 1963). PI became the most remarkable technique for membranes fabrication, where most of the asymmetric porous flat sheet-polymeric membranes made by this technique. The PI process consists of the separation of an initially homogeneous system into two distinct phases, including polymer-rich phase (solid phase) that will give rise to the membrane matrix whereas the polymer-lean phase (solvent rich liquid phase) will originate from the membrane pores. In comparison to other fabrication techniques, PI is extremely versatile and allows high flexibility of membranes material selection, while a wide range of pore sizes can be obtained (1$10000 \mathrm{~nm}$ ) as long as the system (polymer-solvent) miscibility gap over a defined temperature and concentration range (Hilal et al., 2015).

\subsubsection{Phase inversion types}

PI as membrane fabrication technique can be subdivided into four distinguished types, depending on the mechanism exploited to induce the phase separation or what so-called (demixing or precipitation) (Van de Witte et al., 1996), this includes;

i. Evaporation-induced phase separation (EIPS): in this technique, precipitation is induced by the volatile solvent evaporation out of the casting solution. 
ii. Vapour-induced phase separation (VIPS): precipitation occurred when the nonsolvent adsorbed from a vapour phase, which may contain other gasses like nitrogen or air.

iii. Temperature-induced phase separation (TIPS): where precipitation induced by cooling the prepared high-temperature homogeneous polymer solution. The simplest system of TIPS consists two components; a solvent and a polymer.

iv. Nonsolvent-induced or diffusion induced phase separation (NIPS or DIPS): where the nonsolvent bath is exploited to induce the phase separation through immersion of the casting solution in the bath.

In the later technique (NIPS), which is the most commonly used one for preparation of membranes, the casting solution is prepared by dissolution the polymer in an appropriate solvent, then cast on a support plate and finally immersed in a nonsolvent bath to form the membrane. The nonsolvent could be any liquid in which the polymer is not soluble in. Water is the most prevalent one used for polymeric membranes preparation, but other compositions could also be added. The phase inversion will take place due to the diffusion exchange between the solvent and the nonsolvent. This will take the polymer into direct contact with the nonsolvent. Eventually, phase separation of the polymer will occur leading to precipitate the polymer in the nonsolvent to form the membrane (Lalia et al., 2013).

\subsubsection{Parameters affecting phase inversion process.}

During the fabrication of asymmetric polymeric membranes by NIPS technique, many fundamental and secondary parameters can be adjusted to control the overall membrane properties, such as; polymer concentration, type and concentration of additives, evaporation time, casting shear rate, coagulation bath temperature and composition. The fabricated membranes morphology, mechanical strength, permeability and selectivity can be influenced by the interplay of all these preparation parameters. In this section, two of the critical variables (polymer concentration and type of additives) will be covered.

With PI technique, it is possible to control the type of the fabricated membrane process. MF/UF and even NF membranes can be produced by varying the polymer concentration in the casting solution. UF membranes could be obtained from 12-20 it. 
$\%$ polymer whereas higher than $20 \%$ could result in NF (Lalia et al., 2013). Such a drastic change in the morphological characteristics of the membrane would be expected. The use of more concentrated polymer can lead up to the formation of the higher concentrated dope solution at the binodal-phase separation point. Thus, a denser spongious structure would be formed as well as a lesser possibility of solvent extraction occurs from the surrounding polymer solution to the polymer-lean phase during the formation of the microvoids (Blanco et al., 2006).

Regarding the role of additives in the casting solution, a wide range of additives has been used to impart a desirable trait to a candidate membrane. These additives are not only added to act as pore formers but also to improve; hydrophilicity, porosity, surface and antifouling properties of membranes. However, polymeric additives are the most common in which they have diversified chemical natures. Among these agents, hydrophilic polyvinylpyrrolidone (PVP) and polyethylene glycol (PEG) are extensively used to introduce a lesser hydrophobic and a higher permeability membrane with nominal influence on the selectivity (Han \& Nam, 2002; Mosqueda-Jimenez et al., 2004; Ochoa et al., 2001). Moreover, ultimate membranes may have better morphology along with enhanced porosity, pore interconnectivity, and suppressed microvoids formation (Hilal et al., 2015; Wienk et al., 1996). This was ascribed to the capability of PVP to induce the thermodynamic instability of the dope solution and promote the instantaneous liquid-liquid demixing (Feng et al., 2006; Shi et al., 2007). PVP is a water-soluble polymeric additive, and thus, a substantial amount of PVP can readily leach out of the membrane during the solvent-nonsolvent exchange, and to a lower extent during the hydraulic permeation. Indeed, this would result in a gradual deterioration in membranes performance with time (Kim et al., 2005; Wan et al., 2006). Depending on PVP molecular weight used during the fabrication, the stability and the influence on membranes may differ. It has been reported in literature that PVP with lower molecular weight tends to form smaller pores but leaches out more readily from the membranes, than PVP with higher molecular weight. Also, a thicker skin layer contains larger pores is more likely to be formed (Wang et al., 1995, 2000). Usually, PVP leaching might cause a rougher membrane surface in asymmetric membranes than dense membranes (Wan et al., 2006). 


\subsection{Membranes fouling phenomena}

Despite the relatively advanced state of the membrane industry, there are some issues still need to be tackled on the large scale. Most of these impediments can be brought back to one major cause which is the hydrophobic nature of polymeric materials. This would make synthetic membranes more attractive for hydrophobic compounds in water. Indeed, results in "membrane fouling", the main constraint in industrial membrane applications sustainability (Nackaerts, 2014). Particularly, membrane fouling occurs due to the accumulation of various solutes on the membrane surface and/or interior structure of the membrane, forming an additional barrier at the membrane surface or/and blocking the internal pores. Indeed, hindering the solvent from passing through the membrane. This will cause a lesser permeation, a higher transmembrane pressure to maintain same productivity, and shorten membrane lifespan. Moreover, fouled membranes may consume a massive amount of cleaning chemicals or lead up to membrane replacement in severe cases. As a consequence, this will increase the operation and the maintenance costs of the treatment unit (Boussu et al., 2007; Contreras, 2011). Although a good selection of membrane materials, operating design, pre-treatment processes and operating conditions could mitigate the extent of fouling, membrane's sustainability is still intricate matter in the industrial applications and represents a challenging issue due to its variety (Hilal et al., 2012). For several decades, membrane fouling phenomenon has been widely addressed from different angles to minimise its consequences. This includes; understanding its mechanisms, incidence, types, factors affecting fouling growth and corresponding solutions (Becht, 2008).

Generally, foulants may interact differently with membranes depending on many circumstances, as will be addressed later in this chapter. Membranes fouling behaviour can be specified and categorised mainly into two groups; reversible and irreversible fouling. Membranes with reversible fouling can easily restore their original flux by performing the necessary cleaning protocols while releasing the system pressure. In contrast, membranes with irreversible fouling may result in permanent flux decline even after intensive cleaning been performed. Therefore, controlling of foulants adhesion on polymeric membranes surfaces are crucial to mitigate membrane fouling. Disparate strategies have been used to mitigate membrane fouling, this includes, attaching appropriate feed pre-treatment process, ultrasonic entrenchment, cleaning 
chemicals, relaxation, backwash and membrane surface modification (Le-Clech et al., 2006).

\subsubsection{Fouling mechanisms}

Particularly, membranes fouling is extremely complex phenomena that not determined accurately yet (Kimura et al., 2004). According to Hilal et al., (2005), membrane fouling phenomena can take place at different positions in membranes, including both of the selective layer and pore walls. Internal fouling refers to the deposition and adsorption of small particles or molecules onto the pore entrances or the inside of a pore of the membrane whereas external fouling refers to the accumulation of rejected molecules upon the membrane surface. So, understanding the contributing fouling process mechanisms could provide better means to minimise membranes fouling subconsequences. According to W.R. Bowen (1995), these mechanisms can be categorised into four physically based types (Figure 2.3), as follows:

i. Pore plugging (complete blocking of the pores): this type of fouling occurs when each particle at the membrane surface has fully blocked one or more pores without any particles superposition.

ii. Long-term adsorption (intermediate blocking): in this type, subsequent particles have accumulated on the beforehand arrived particles that formerly blocked some pores or directly blocked other pores.

iii. Direct adsorption (standard blocking): As a consequence of this fouling type, a decrease in the pore volume will occur due to the deposition of particles on the wall of the internal pore in the membranes.

iv. Cake filtration (boundary layer resistance): this fouling results from adhesion of new particles on one or more formerly arrived foulant particles which had already blocked some of the membrane pores. However, there is no direct deposition between the membrane surface and these newly attached foulants. 


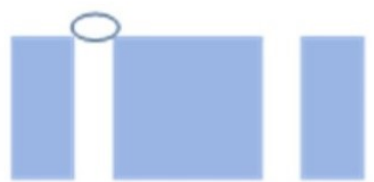

$\mathbf{A}$

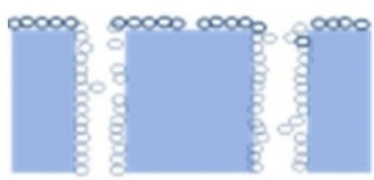

C

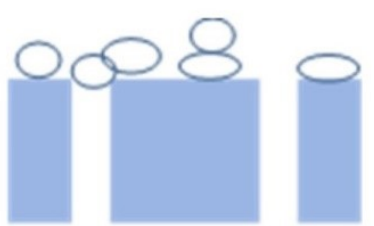

B

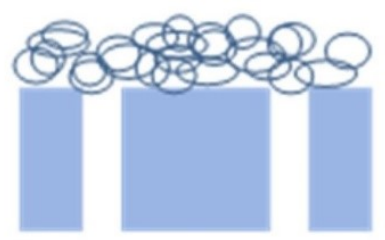

D

Figure 2.3: Fouling mechanisms; (A) complete blocking, (B) intermediate blocking, (C) standard blocking, and (D) cake blocking.

In fact, membrane's fouling may combine some or all of these mechanisms during the fouling formation process depending on the membrane characteristics. Thus, membrane fouling behaviour in the four pressure driven processes is not equivalent. In UF and MF (loose membranes), all the previously mentioned mechanisms might be involved to take part in the formation of final fouling configuration, while cake formation and concentration polarization are more likely to foul RO and NF membranes, as their fouling was assumed to take place on the membrane surface due to their tightness and relatively the size of foulants in comparison to membrane pore sizes (Boussu et al., 2007).

\subsubsection{Foulants categorisation}

Depending on foulant's nature, foulants were categorised into four major types; scale, silt, organic and biological fouling.

Scaling can be defined as the nucleation and growth of inorganic minerals on the surface of membranes (Antony et al., 2011), and is responsible for the surface blockage and flux decline of the RO/NF membranes. During the pressure-driven desalination processes, the sparingly soluble inorganic (e.g. $\mathrm{CaSO}_{4} \cdot 2 \mathrm{H}_{2} \mathrm{O}, \mathrm{CaCO}_{3}$, and $\left.\mathrm{Ca}_{3}\left(\mathrm{PO}_{4}\right)_{2}\right)$ salts become supersaturated and may precipitate on a membrane surface to cause scaling, that will end up with many environmental and economic issues (Shirazi et al., 
2010; Van de Lisdonk et al., 2000). The concentration of dissolved minerals tends to build up by the factor of $4-10$ (Antony et al., 2011). Major factors that can dictate the severity of scaling are surface morphology of membranes, operating condition, and kinetics of crystallisation and precipitation (i.e., surface and bulk crystallisation) (Goh et al., 2018).

Whereas, organic fouling can be defined as the combination of deposition, reaction and interaction of high molecular weight organic molecules (e.g. transparent exopolymer particles (TEPs) and/or natural organic matters (NOM)) with the membrane surface (Goh et al., 2018). Membranes fouling with naturally occurring organic materials has frequently cited as the primary cause of chronic fouling in membrane processes. The factors that predetermine the relative propensity of organic materials to foul a membrane include their molecular weight, conformation, membranes surface characteristics and the organic foulant affinity for the membrane (Devitt).

Biofouling was defined as the microbial cell adhesion and the later colonisation at the membrane surface forming microbial biofilm (Zhang et al., 2012). Typically, membrane biofouling caused by biologically active organisms (e.g. viruses, fungi, and microorganisms) is the most complicated form of fouling among the types above, since these organisms tend to adhesively attach, accumulate and colonise at the membrane surface (Kochkodan \& Hilal, 2015). Commonly, this microbial attach to a membrane surface is initialised by the irreversible adhesion of one type of bacteria or more and followed by the subsequent growth and multiplication of the sessile cells in the presence of feed water nutrients (Upen et al., 2000). Once they attach the surface and form a matrix of extracellular polymers, their removal will be quite hard even with applying biocides (Subramani \& Hoek, 2008). In general, physiochemical parameters such as solution chemistry, hydrodynamic and interfacial forces can influence the biofouling behaviour in polymeric membranes. Also, biofouling can be impeded by making the membrane surface more hydrophilic or negatively charged, (Lee et al., 2013).

Lastly, colloidal (silt) fouling could result from the accumulation of suspended and colloidal particles having a size ranging from few nanometers to few micrometres (e.g. silica, iron hydroxides/oxides, heavy metals hydroxides and organic colloidal matters) (Potts et al., 1981). 


\subsubsection{Factors affecting membranes fouling}

Diversified linked factors can critically act for accretion or diminishing of fouling phenomena in polymeric membranes applications. In addition to the chemical and physical pretreatments, there are leading factors could heavily influence the fouling behaviour of any membrane; these are (i) membranes characteristics, (ii) hydrodynamic conditions and (iii) feed solution chemistry.

\subsubsection{Membrane characteristics}

According to (Al-Amoudi \& Lovitt, 2007; Rana \& Matsuura, 2010), improving membranes surface properties can efficiently reduce fouling and enhance the overall performance. So far in the literature, considerable strategies, with a view for enhancing membrane surface characteristics, are aimed for smoothing, hydrophilization and attaching charged functional groups to the membrane surface to diminish undesired membrane-foulant interactions. Basically, membrane surface characteristics consist of surface roughness, hydrophilicity and surface charge.

\section{i. Surface roughness}

Membrane's surface roughness is one of the most critical features that directly linked to membrane flux and fouling (Zhu \& Elimelech, 1997). A rougher membrane's surface is believed to be more favourable to foul. This is attributed to the higher surface area presented to where foulants can be attached. Preferentially these foulants tend to accumulate in the valleys of rougher surfaces and clog them, resulting in more severe fouling and faster fouling rates (Vrijenhoek et al., 2001). Additionally, it is pointed out that water current during the cross-flow filtration can act as a shear force to prevent foulants from attaching the membrane surface. Indeed, smooth membranes would not readily foul, while the influence of this shear force is supposed to be minimal at rougher surfaces (Geesey et al., 1996).

\section{ii. Hydrophilicity/hydrophobicity}

Membrane's material hydrophobicity/hydrophilicity is another characteristic that can noticeably affect membrane fouling behaviour. Practically, hydrophilicityhydrophobicity of a membrane surface is based on contact angle measurements where a lower contact angle refers to a higher hydrophilicity (Hilal et al., 2015). Therefore, a membrane prepared from hydrophilic materials is expected to be less fouled than membranes made from a hydrophobic one (Hilal et al., 2012). 
According to Israelachvili (1992), membranes prepared from hydrophilic materials are more attractive to water molecules where these molecules tend to form hydrogen bonds with the membrane materials and itself. As a consequence of water molecules attraction between each other, more water would be adsorbed, and a water layer will be formed at the membrane surface reducing the membrane-foulant attractions. Moreover, water layer thickness is believed to increase proportionally with an increase in membranes material hydrophilicity. On the other hand, lesser hydrophilic membranes (contact angle is no greater than $90^{\circ}$ ) have a lower tendency for water molecules adsorption, and once they are fully wetted, a water layer will also form. In the meantime, less hydrophilicity surfaces can be subjected to secondary impacts make them more prone to pores blockage and internal foulant adhesion by hydrophobic molecules presented in the feed solution, resulting in a lower permeate flux (Jönsson \& Jönsson, 1995). Thus, hydrophobic molecules tend to avoid attachment to membranes have hydrophilic surfaces which explain why hydrophilic membranes are less prone to fouling than hydrophobic one.

Membranes surface modification is becoming a growing field of research since it is believed that turning membrane surface properties through incorporating new hydrophilic functional groups would produce more hydrophilic surfaces (Wang et al., 2012). (Koehler et al., 2000), studied the intermolecular forces between a modified polysulfone (PS) membrane and the protein Lysozyme (LYZ), results confirmed that hydrophilic membranes not only showed lower fouling and contact angle but also reduced an amount of materials adsorbed and adhesion forces. (Kochkodan et al., 2006) prepared different composite membranes with chemically different surfaces via the photochemical modification. Membranes with hydrophilic surfaces exhibited less susceptibility than hydrophobic membranes to microbiological fouling using E.coli suspension.

Currently, there is no experimental method capable of measuring the hydrophilicity as a unique property of the membrane chemistry. Rana \& Matsuura (2010) reported that even though contact angle has been used to express membranes hydrophilicity, it could be affected by surface morphology (e.g. surface roughness and pore size). Fouling reduction in hydrophobic membranes suggests that membrane fouling is affected by chemistry parameters other than hydrophilicity. For instance, the solution $\mathrm{pH}$ can 
influence the foulants characteristics (e.g., size, shape and surface charge), and ultimately the interactions between the membranes and molecules.

\section{iii. Surface charge}

Surface charge characteristics of a membrane may accelerate or inhibit the fouling phenomena in polymeric membranes since it may behave as absorption sites for foulants (Ikeda et al., 1988). Indeed, making the membrane more susceptible to foul with charged foulants presented in the feed solution, when both the membrane and solutes have different charges (Gerard et al., 1998). In contrast, deposition of a solute on a membrane surface could be reduced when both the solute and the membrane have similar charge (Elimelech et al., 1994; Van der Bruggen et al., 2008). (Becht, 2008) claimed that introducing charged groups on a polymeric membrane surface improve not only the separation performance but also reduce the deposition of foulants. With no thoughtfulness to membrane surface roughness and hydrophobicity, a reduction in membrane total fouling might be achieved as a consequence of these repulsion forces indeed (Abu Seman, 2010). While the role of surface charge characteristics tends to be marginal at a higher membrane surface roughness due to the higher tendency of foulants adsorption. (Reiss et al., 1999) showed that a negatively charged membrane with higher surface roughness exhibited greater organic fouling than less negatively and smoother membranes. Furthermore, deposition of these NOM was reported on membrane surface in some cases, especially when the hydrophobic interactions exceed NOM-membrane charge repulsion forces (Tu et al., 2005).

\subsubsection{Hydrodynamic conditions}

Hydrodynamic conditions including; module geometry, type of flow and operating pressure are significant factors with the view to alleviate membranes fouling behaviour in industrial applications. The extent of fouling formation/accumulation on the surface of a membrane is strongly subjected to the geometry of that membrane. Therefore, industrial flat sheets and/or hollow fibres membranes are packaged into efficient and economic modules, aiming to maximise their active contact area with feed solutions (Baker, 2004). The spiral wound is one of the widely used industrial modules due to its high packaging density to size employed in the design. The incorporated spacers into the module design along with the tangential flow across the membrane surface are efficient routes to enhance the surface hydrodynamically. During the cross-flow 
filtration, a lower accumulation of foulants on membrane surface could be produced since water current could act as a shear force preventing the growth of fouling layers. Furthermore, retentate side can be recycled back to the feed solution side for further filtration, in contrast to the perpendicular flow in dead-end filtration where the filtrate is limited to permeate flux. Moreover, operating pressure could also influence fouling behaviour. Even though a higher operating pressure could result in a higher permeate flux, it may create additional drag force and compressed fouling layers on the operating expenses of the high flux produced (Seidel \& Elimelech, 2002).

\subsubsection{Feed solution characteristics}

Generally, each foulant has a specific surface charge, particle dimensions, and hydrophobic nature. These characteristics may be significantly influenced by feed solution conditions (e.g. $\mathrm{pH}$, ionic strength and salt type), along with their influence on membrane surface characteristics (Mo et al., 2008; Tang et al., 2007). Solution chemistry can have an impact on foulant-foulant interactions from one side, and foulants-clean membrane surface from the other side. Fouling could be the harshest when filtering a solution in which foulants have low stability and weak interactions (Tang et al., 2007). For instance, fouling produced by hydrophilic uncharged molecules would be least at $\mathrm{pH} 7$ while negatively charged molecules could pursue better at high pH (Boussu et al., 2008). In another research, (Mo et al., 2008) reported that the most significant flux decline, during the filtration of Bovine serum albumin (BSA) through $\mathrm{RO}$ membrane, was achieved at a $\mathrm{pH}$ value close to the isoelectric point of BSA. This was attributed to the aggregation resulted from the weakened electrostatic repulsions of BSA molecules. While charge shielding and double layer compression effects were enhanced at higher ionic strength, producing weaker electrostatic repulsions and severer fouling conditions. In fact, proteins are known as polymers built up from different amino acids, and their functional groups that dictate the charge are located at the outer edges of their structure. Hence, that charges might be altered when changing the solution environment $(\mathrm{pH})$ in addition to the membrane surface charge itself (Becht, 2008). 


\subsection{Membrane modification}

Membrane modification is defined as the process of incorporating a hydrophilic functional group to the surface of a membrane, aiming to enhance the free surface energy and thereby reduces fouling. In fact, membrane separation processes are surface dependent, where the membrane's active layer (skin) controls the separation process and the membrane-foulant interactions. Indeed, introducing a hydrophilic functional group to that surface was believed to improve the separation performance of this membrane and to reduce/control the undesired adhesion and/or adsorption interactions between foulants and that active layer (Kochkodan et al., 2014). For achieving this goal, an assortment of methods has been suggested and could be used individually or as a combination of more than one technique (Van der Bruggen, 2009), this includes; grafting (Helin et al., 2008), surface chemical reaction (Wang et al., 2006b), blending (Wang et al., 2006c; Zhao et al., 2011b), plasma treatment (Yu et al., 2005a), dip coating (Madaeni \& Ghaemi, 2007), ion implantation (Mukherjee et al., 2005b). However, each technique has its pros and cons.

One of the most versatile modification techniques is surface grafting, where a monomer could covalently have bonded onto a polymer chain. This can be achieved by photo-irradiation, chemical treatment or high-energy radiation technique (Sathish Kumar et al., 2015). Different molecules could be attached to the membrane surface, such as amphiphilic copolymers (Asatekin et al., 2006) and zwitterionic polymers (Zhao et al., 2011a). However, even though it has been approved as successful modification technique and considered as stable technique, its practical applications are still doubtful where some chemicals used in grafting are environmentally unfriendly. Furthermore, grafting by UV irradiation, gamma ray, and plasma is difficult to apply in large industrial applications (Rana \& Matsuura, 2010).

Nevertheless, formation a layer on a membrane surface via dip coating and adsorption is one of the cheapest modification techniques, since no costliest equipment are required (Sathish Kumar et al., 2015). However, the surface layer durability is questionable in membranes industrial applications since these layers tend to degrade gradually during the operation (Khulbe et al., 2010; Rana \& Matsuura, 2010). In addition to the potential of coating layer delamination, this layer may cause significant undesirable changes in membrane permeability as a consequence of pore plugging or narrowing, especially when modification by NM been applied (Razmjou et al., 2011). 
Likewise, blending of various additives with the polymeric matrix is widely applied due to its simplicity and effectivity. A variety of NMs, polymeric additives, inorganic compounds...etc., can be added during the membrane fabrication process, where no extra steps and instruments to generate radiation or plasma are required, unlike the more complex modification techniques. Only an additional component is added to the casting solution to produce a membrane as desired (Nackaerts, 2014). These additives are not only able to enhance the membrane hydrophilicity but also could influence the structure of the membrane (Wienk et al., 1996). However, in this method, additives are impregnated in the inner structure of the membranes other than concentrated on the surface. Indeed, their functionalities are partially exploited (Hilal et al., 2015).

\subsubsection{ENMs for polymeric membrane applications.}

Recently, the implication of ENMs into a polymeric membrane matrix have gained extraordinary attention in water and wastewater treatment applications (Wang et al., 2013). Due to the variety of polymeric membranes structures and properties, inexpensiveness of polymers, ease of preparation, integration to different configurations and pore formation control, polymeric membranes are still more preferable in industrial applications. In the meantime, the hydrophobic nature of polymers, as well as their limited thermal, mechanical and chemical resistance, represents the main obstacles in membrane industry (Ulbricht, 2006; Xu et al., 2009). Differently, inorganic membranes have the much higher mechanical strength and chemical resistance, while their applicability for water treatment purposes is quite restricted due to high fabrication costs and preparation difficulties ( $\mathrm{Ng}$ et al., 2013). Therefore, a vast number of studies has been devoted for producing what so-called "hybrid" or "nanocomposite" membrane using various NMs.

Nanocomposite membranes, as a new class of membranes, consists both organic polymers and inorganic nanoscale materials, are believed to exhibit enhanced performance in comparison to nascent membranes (Kango et al., 2013; Qu et al., 2013). The membrane that merges the synergic effects for both organic and inorganic materials would have a new entity with enhanced hydrophilicity, permeability, thermal and chemical stability, porosity and mechanical properties (Kumar et al., 2009; Zhao et al., 2013). In the meantime, many issues may arise from incorporation of nanomaterials 
into polymeric membranes that might have disruptive consequences on these membranes and the environment. Indeed, choosing convenient nanomaterials with an optimum composition is an essential aspect to overcome their limitations in polymeric membrane applications ( $\mathrm{Ng}$ et al., 2013).

For water treatment applications, a vast number of studies on coupling nanoscale materials with polymeric membranes have focused on (i) carbon-based materials, for instance; Graphene oxide (GO) (Xia \& Ni, 2015; Xu et al., 2014) and carbon nanotubes (CNTs) (Celik et al., 2011b; Shen et al., 2013; Vatanpour et al., 2011), (ii) metal and metal oxide nanoparticles like; Silver (Ag) (Kim et al., 2012; Prince et al., 2014), Titanium $\left(\mathrm{TiO}_{2}\right)$ (Li et al., 2009; Rahimpour et al., 2008; Shi et al., 2013), Aluminum $\left(\mathrm{Al}_{2} \mathrm{O}_{3}\right)$ (María Arsuaga et al., 2013), Silicon $\left(\mathrm{SiO}_{2}\right)$ (Yu et al., 2009), Iron $\left(\mathrm{Fe}_{3} \mathrm{O}_{4}\right)$ (Alam et al., 2013; Xu et al., 2012), Zirconium ( $\left.\mathrm{ZrO}_{2}\right)$ (Maximous et al., 2010) and Zinc (ZnO) (Balta et al., 2012), (iii) alumina-silicate based nanomaterials like; clay nanoparticles (Mierzwa et al., 2013) and zeolite (NaX) (Fathizadeh et al., 2011). (iv) in addition to an assortment of $\mathrm{NM}$, for instance; Calcium carbonate $\mathrm{CaCO}_{3}$ (Nair et al., 2013).

\subsubsection{Special features of ENMs}

During the last two decades, materials and structures, manifesting geometric dimensions below $100 \mathrm{~nm}$, have inspired the scientific world (Hanemann \& Szabó, 2010). Different nanomaterials synthesised by various techniques have been applied in many fields, including medical supplies, pigments, cosmetics production, catalysts, toner and ink (Okada \& Usuki, 1995). Nanomaterials are classified under different criteria, depending on the applications, materials, and fields concerned. However, a widely accepted definition of nanoparticles is that they are particles with a diameter $<10-20 \mathrm{~nm}$; a size with a surface area to volume ratio where a drastic change in the physical behaviour of the materials occurs. Moreover, in many cases, particles with size ranging from 1 to $100 \mathrm{~nm}$ are also referred to as nanoparticles (Hosokawa et al., 2007). In a narrower scene, based on their dimensionality, nanoscale materials are divided into four broad categories: zero-dimensional (0D), one-dimensional (1D), two-dimensional (2D) and three-dimensional (3D). 0D nanoscale materials include uniform particles arrays, heterogeneous particle arrays, core-shell quantum dots, onion-like layered particles, nano lenses and hollow spheres (Figure 2.4). 1D includes nanorods, 
nanowires, nanobelts, nanotubes and hierarchical nanostructures (Figure 2.5). 2D is composed of nanoplates, nanosheets, branched structures, nanoprisms, nanowalls, and nanodisks (Figure 2.6). Lastly, 3D nanostructures include nanocones, nanocoils, nanopillers, and nanoflowers (Tiwari et al., 2012) (Figure 2.7).

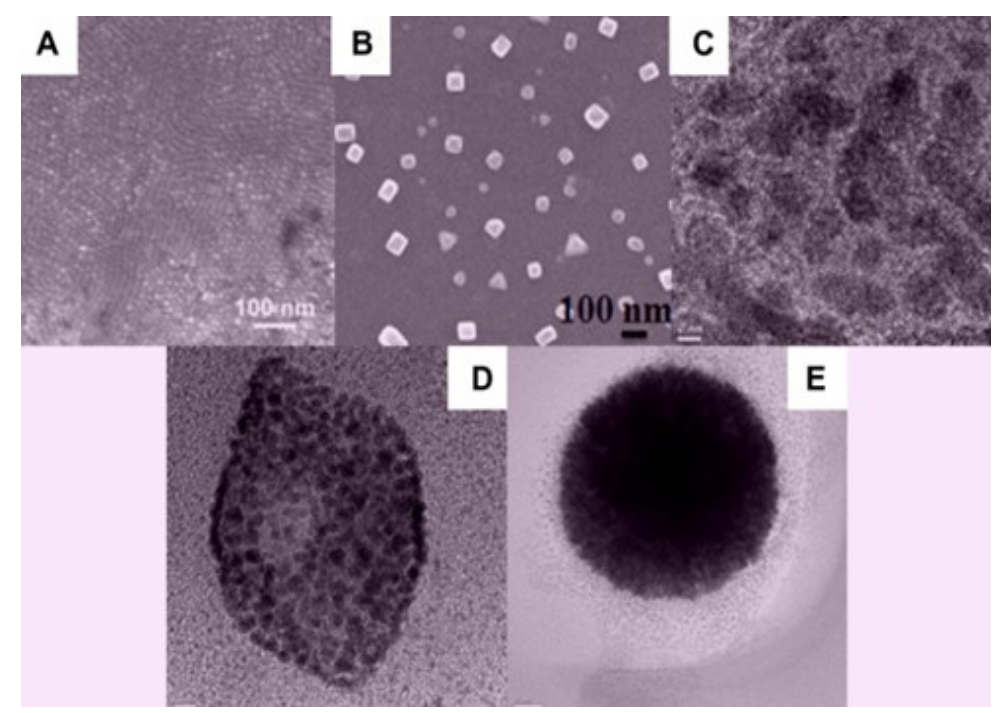

Figure 2.4: SEM and TEM images for various zero-dimensional (0D) ENMs. (A) Quantum dots, (B) nanoparticles arrays, (C) core-shell nanoparticles, (D) hollow cubes and (E) nanospheres. Adopted from (Tiwari et al., 2012).
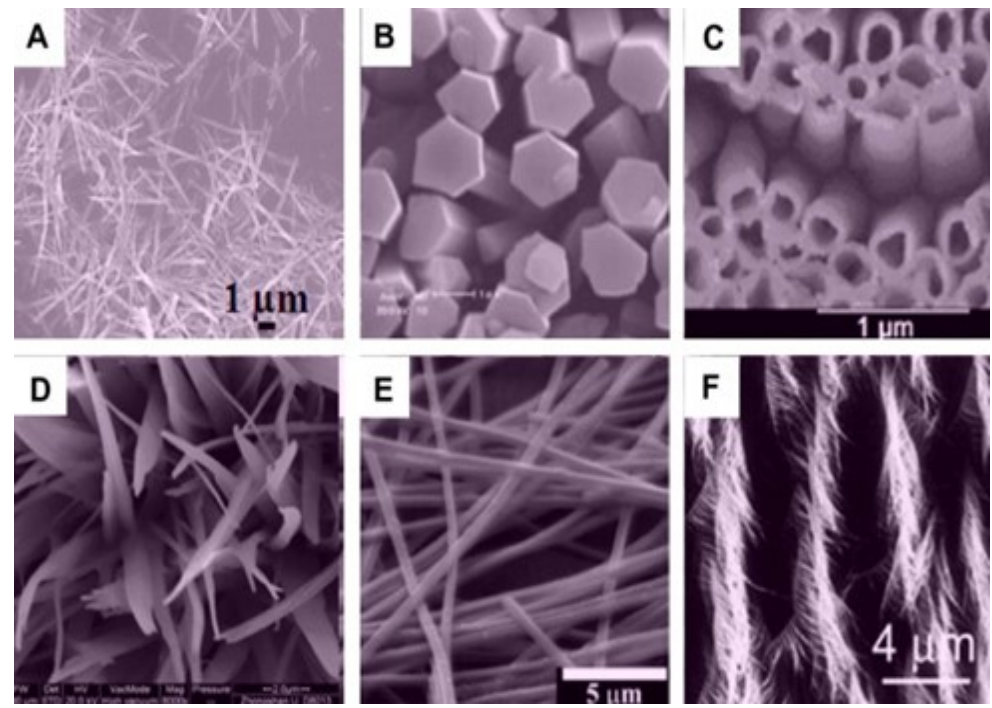

Figure 2.5: SEM and TEM images for various one-dimensional (1D) ENMs. (A) Nanowires, (B) nanorods, (C) nanotubes, (D) nanobelts, (E) nanoribbons, and (F)hierarchical nanostructures. Adopted from (Tiwari et al., 2012). 


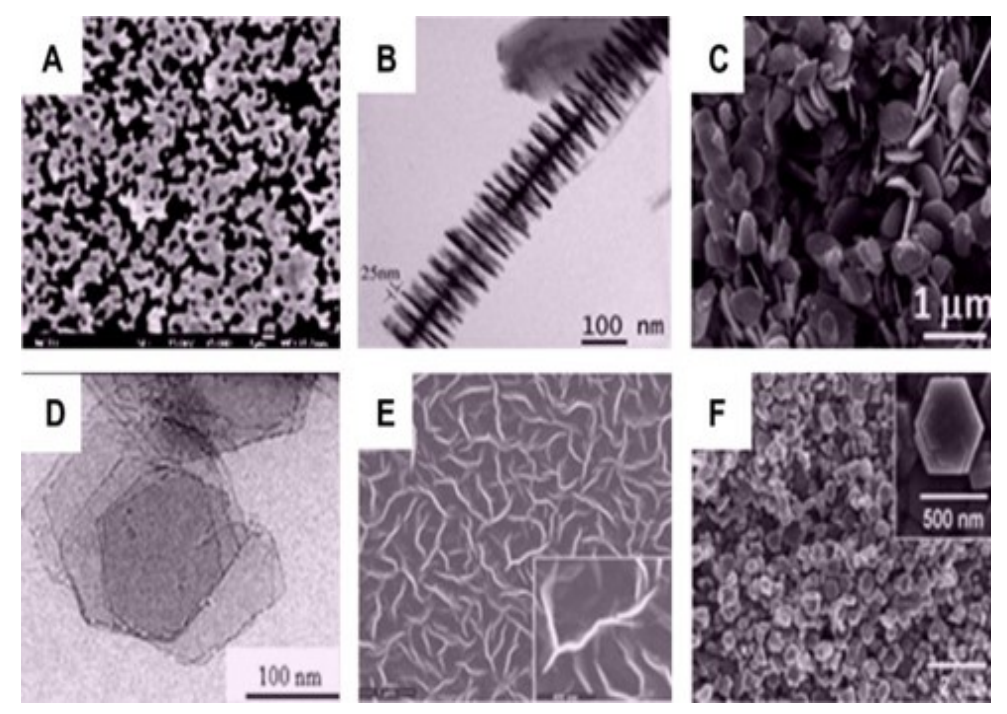

Figure 2.6: SEM and TEM images for various two-dimensional (2D) ENMs (A)

Junctions (continuous islands), (B) branched structures, (C) nanoplates, (D) nanosheets, (E) nanowalls, and (F) nanodisks Adopted from (Tiwari et al., 2012).
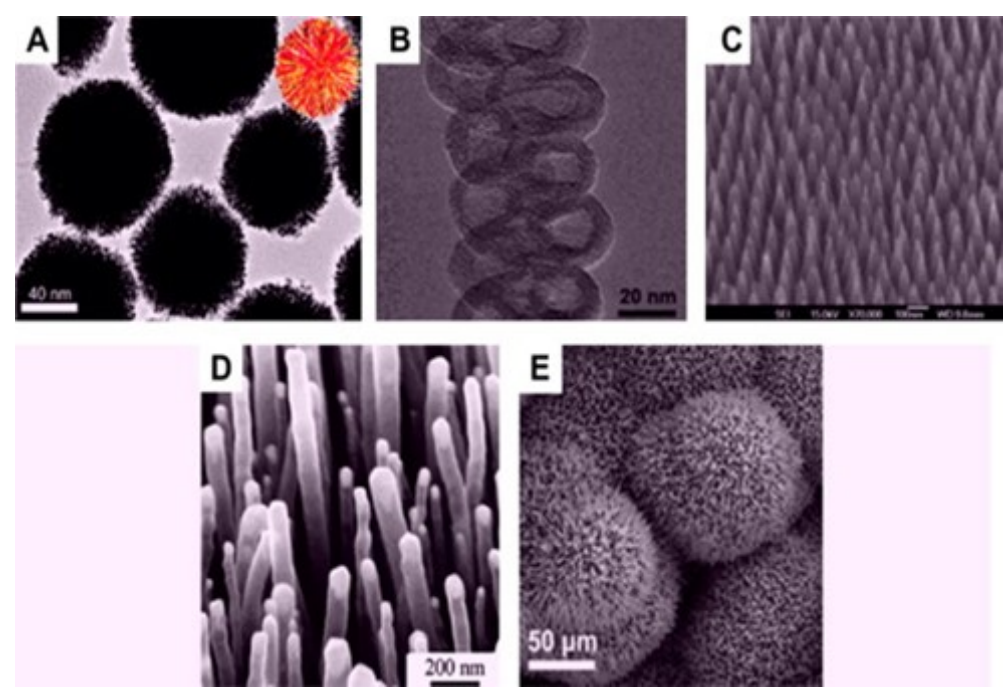

Figure 2.7: SEM and TEM images for various three-dimensional (3D) ENMs. (A) Nanoballs, (B) nanocoils, (C) nanocones, (D) nanopillers and, (E) nanoflowers. Adopted from (Tiwari et al., 2012).

As material is distributed as smaller particles within a system (and the surface area to volume ratio of particles increases) the proportion of surface atoms increases resulting in a higher active surface area, e.g. $5 \mathrm{~nm}$ particles compose only a few $10^{3}$ of atoms or unit cells, and possess about $40 \%$ of the atoms at the surface. While $0.1 \mu \mathrm{m}$ particles compose $10^{7}$ atoms or unit cells and possess only $1 \%$ of the atoms on the surface (Hanemann \& Szabó, 2010). The increase in the number of the interfacial atoms 
of material distributed within a system as nanoparticles mean that their behaviour at the interface will dominate the behaviour of the bulk material within the system. This behaviour is manifest for nanoparticles as differences in optical, magnetic, thermodynamic, thermomechanical, electronic and structural properties. Consequently, the desired properties of the aimed nanocomposite might show enhanced electrical, thermal, mechanical and rheological properties depending on the size, shape, composition of these nanomaterials and their interactions with the host polymeric matrix (Kango et al., 2013).

\subsubsection{Strategies for incorporating ENMs into polymeric membranes}

In general, two strategies have been adopted to prepare nanocomposite membranes; thin film nanocomposite membranes, where ENMs have been deposited on the surface of a polymeric membrane, and mixed matrix nanocomposite membranes fabricated through the direct entrapment of ENMs within the polymeric matrix (Jhaveri \& Murthy, 2016). A combination of both techniques has also been examined (Kim et al., 2012). However, to enable the fabrication of novel multifunctional nanocomposite membranes avoiding complex processes, both coating/deposition and blending can be used to achieve a broad range of membranes with diverse properties that can be bespoke for the desired application.

Coating/deposition is the process of forming a layer of ENMs on the active surface layer of a membrane, aiming to control the hydrophilicity of the membrane surface through altering the chemical groups that are displayed at the surface (Sathish Kumar et al., 2015). The most straightforward and economical technique is by introducing ENMs to the membrane surface by self-assembly. Self-assembly is based on immersion of a membrane or its active layer in a diluted-colloidal solution of ENMs. There is a spontaneous association of ENMs with the membrane material. The thickness of the fabricated layer depends on the exposure time and ENMs concentration used during the modification process. However, the self-assembly process is only applicable for certain ENMs that have sufficiently strong interaction with polymeric materials (Kim \& Van der Bruggen, 2010; Rahimpour et al., 2008). (Li et al., 2009) prepared novel $\mathrm{TiO}_{2}$ nanocomposite membranes via electrostatic self-assembly, based on an ultrahigh molecular weight poly(styrene-alt-maleic anhydride)/poly(vinylidene fluoride) (SMA/PVDF) blend membrane. The SMA/PVDF blend membranes prepared by the 


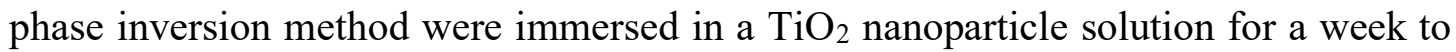
produce the $\mathrm{TiO}_{2}$ self-assembly membranes. An extension of this method used in coating membrane surfaces with ENMs is the layer by layer technique (LBL), which has shown great potential in the fabrication of nanocomposite membranes since it was introduced to prevent the destruction of self-assembled layers (Hilal et al., 2015). LBL assembled layers can introduce further binding sites for ENMs and create membrane surface multi-functionalization (Xu et al., 2015). A further method, grafting, has also shown promising results. (Trejo \& Frey, 2015) conducted a comparative research study that immobilised carboxylic acid-coated $\mathrm{Fe}_{2} \mathrm{O}_{3}$ nanoparticles on the surface of Nylon 6 membrane via three techniques: (1) simultaneous electrospinning /electrospraying, (2) LBL and (3) chemical grafting. These researchers only found uniform dispersion of the nanoparticles with electrospraying and grafting even though good bonding interaction control between ENMs was observed for all of the membranes (Figure 2.8). In another study, (Mauter et al., 2011) applied biocidal Ag nanoparticles via a post-fabrication grafting technique. The authors claimed that grafting could maximise the density of ENMs at the surface and provide efficient routes for fabricating reactive nanocomposite membranes.
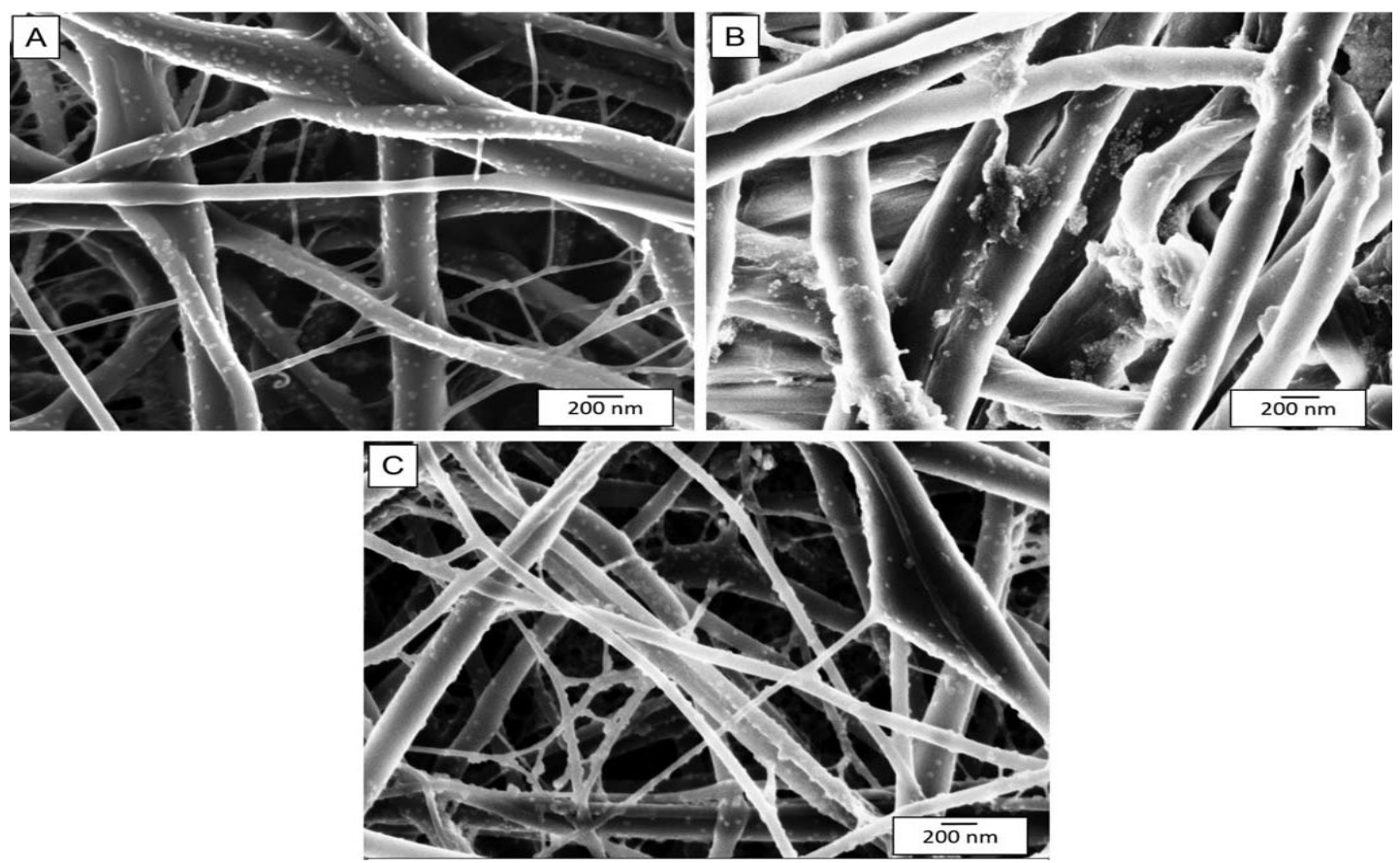

Figure 2.8: Comparison of FE-SEM images (A) simultaneous electrospin/electrospray, (B) LBL, and (C) grafting (Mauter et al., 2011). 
In contrast to the previously discussed techniques, ENMs blending or bulk addition can be achieved during membrane synthesis processes based on phase inversion. This results in ENMs that are impregnated within the inner structure of the membranes other than concentrated on the surface. Therefore, their functionalities and influence on the separation processes are partially exploited due to the shielding of ENMs by the embedding polymer matrix (Kim \& Van der Bruggen, 2010). However, these ENMs could influence the skin (pore size and pore size distribution) and the support layer morphologies for the asymmetric porous nanocomposite membranes. Membrane selectivity, permeability and compaction behaviour and change in membrane morphology are reported to be a function of ENMs type, loading density and their method of incorporation (Tarabara, 2014). Various membrane surface and inner morphologies can be tailored as desired by finely tuning these parameters, as will be seen in the next section.

\subsubsection{Influence of various ENMs on morphological and antifouling properties of nanocomposite membranes}

Numerous ENMs are available for the incorporation into polymeric membranes to create innovative solutions that mitigate fouling, achieve high sustainable fluxes and improve the chemical and physical response of the membrane material. This review now focusses on the most promising and investigated ENMs that have been used for fabrication of composite membranes for application in water treatment.

\subsubsection{Metal/metal oxides based nanoparticles}

I. Silver-based nanocomposite membranes

Silver nanoparticles (Ag-NPs) have gained special interest due to their unique characteristics that include, antimicrobial, optical and electrical properties (Iravani et al., 2014; Li et al., 2017) (Figure 2.9). So far, a wide variety of physical (Henglein, 2001), chemical (Guzmán et al., 2009; Panáček et al., 2006) and biological (Medda et al., 2014; Song \& Kim, 2009), techniques have been introduced to synthesize Ag-NPs with a range of characteristics. Ag-NPs have a broad range of applications which include electronics, bio-sensing, clothing, food industry, paints, sunscreens, cosmetics and medical devices (Ahamed et al., 2010). 


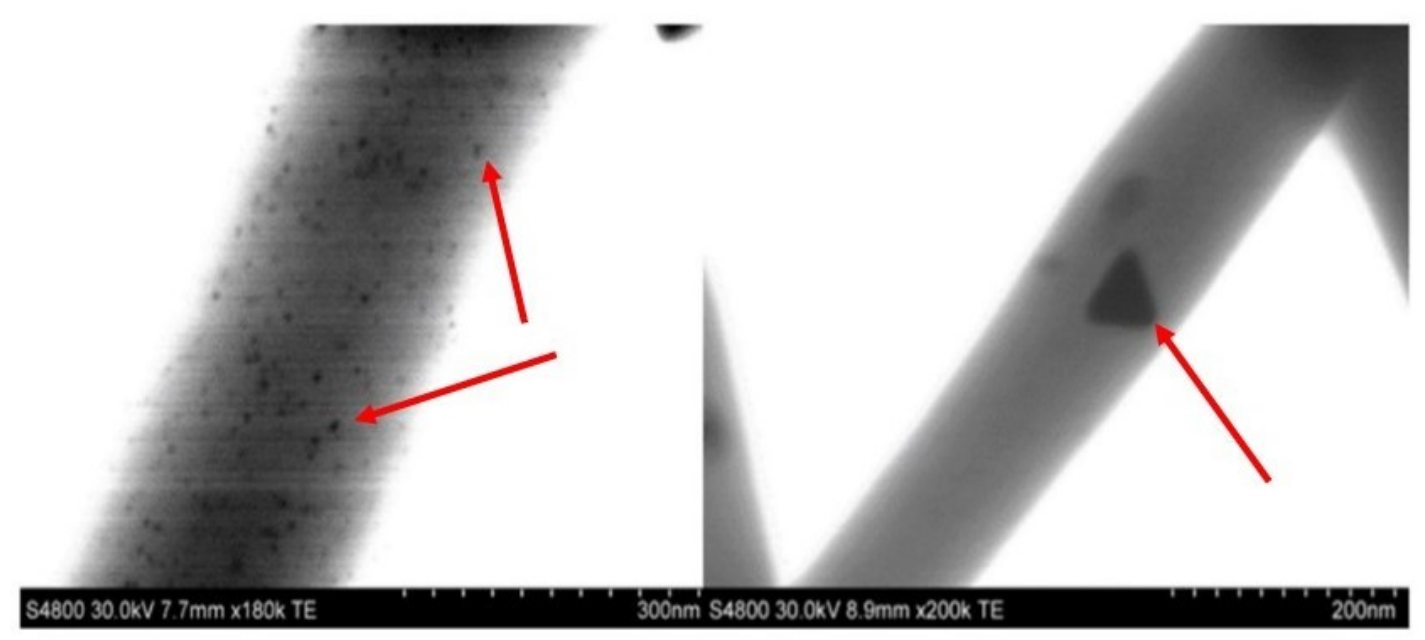

Figure 2.9: SEM images of silver nanoparticles incorporated into electrospun membrane filters (PEO). The right-hand image demonstrates the incorporation of triangular silver nanoparticles.

A key feature of Ag-NPs is their antibacterial activity, which is a highly desirable trait that they could bestow on a membrane. This is a major challenge in water treatment applications, which require alternative disinfection technologies, without forming harmful disinfection byproducts an issue for many of the conventional costly chemicals that currently dominate the industry (Li et al., 2008). From this point of view, and growing experience within nanotechnology and improved capabilities in the fabrication of ENMs with biocidal activity, new opportunities exist for the development of novel antimicrobial membranes. This is particularly pertinent to the development of composite membranes as Ag-NPs are considered to be the most prevalent bactericide of ENMs (De Matteis et al., 2015).

The antimicrobial effects of silver ions $\left(\mathrm{Ag}^{+}\right)$or salts are well recognised, but the effects of Ag-NPs on microorganisms and the antimicrobial mechanisms are not comprehensively understood (Kim et al., 2007). The major obstruction now is deciphering whether the biocidal activity is attributed to the direct exposure to Ag-NPs, or to dissolved silver ions $\left(\mathrm{Ag}^{+}\right)$released from $\mathrm{Ag}-\mathrm{NPs}$ in an aqueous environment, or likely, a combination of both mechanisms may also be responsible (Johnston et al., 2010; Kim et al., 2009; Tolaymat et al., 2010). (Navarro et al., 2008) tested the toxicity of Ag-NPs against algae, the research concluded that Ag-NPs functioned as a source of $\mathrm{Ag}^{+}$, while only minimal toxicity was attributed to the Ag-NPs alone. Similarly, $\mathrm{Ag}^{+}$ was found to be the main source for Escherichia coli inactivation (Kim et al., 2008). 
(Mauter et al., 2011) linked the bactericidal efficiency of Ag-NPs to the release of silver ions and their residual concentration. In contrast, (Fabrega et al., 2009) reported poor antibacterial activity for $\mathrm{Ag}^{+}$and thus, the toxicity was mostly attributed to the direct contact with Ag- NPs. (Sotiriou \& Pratsinis, 2010) and (Foldbjerg et al., 2009) claimed that both $\mathrm{Ag}-\mathrm{NPs}$ and $\mathrm{Ag}^{+}$ions could have antibacterial and cytotoxicity effects, respectively. (Li et al., 2008) suggested that the antimicrobial mechanism of Ag-NPs was due to the disruption of the cell membrane functions and electron transport chains as well as damage to proteins and DNA. (Yin et al., 2013) reported that Ag-NPs could inhibit the growth of microbes through multiple pathways: (i) release of $\mathrm{Ag}^{+}$ions to interact with disulfide or thiol groups of enzymes or DNA, then disrupt the microbes metabolic processes, generate reactive oxygen species (ROS) or interrupt replication of DNA; (ii) affect the cell integrity and metabolism by direct interaction with cell membranes of microbe species; (iii) Ag- NPs having size below $10 \mathrm{~nm}$ could penetrate into bacteria and cause further damage through interacting with DNA. Another investigation claimed that Ag-NPs might behave as a "Trojan horse" by penetrating the cell membrane, then releasing $\mathrm{Ag}^{+}$to cause cytotoxicity (Park et al., 2010). In addition, a number of studies have linked the antimicrobial activity of Ag-NPs to their physiochemical properties. (Pal et al., 2007) claimed that Ag-NP activity is shape dependent, where truncated triangular Ag-NPs showed better antibacterial effects than rod-shaped and spherical nanoparticles against the gram-negative bacterium E. coli. Whereas Ag-NP's size is found to be the basic determinant for their toxicity, smaller Ag- NPs appeared to have a greater antimicrobial activity than bigger Ag-NPs due to the greater contact surface area of the material with the bacteria (Choi \& Hu, 2008). Also, (Xiu et al., 2011) found that smaller nanoparticles ( $8 \mathrm{~nm})$ exhibited better bactericidal effects than larger nanoparticles (11-23) $\mathrm{nm}$ against E. coli. Similar observations have been reported in other studies (Johnston et al., 2010; Sotiriou \& Pratsinis, 2010).

Ag-NPs have been widely used to prepare novel nanocomposite membranes with enhanced pure water flux, antifouling and self-cleaning properties with imperceptible or no effect on the membrane's selectivity. According to Zodrow et al. (2009), Ag-NPs impregnated into UF/PS membrane showed significant bactericidal effects to two species of bacteria; Pseudomonas mendocino and E. coli. The antimicrobial activity was mostly attributed to $\mathrm{Ag}^{+}$release. Moreover, the nanocomposite membrane 
restrained the bacterial attachment to the surface and inhibited biofilm growth. In addition, incorporation Ag-NPs enhanced the membrane surface hydrophilicity, water flux and mitigated other types of fouling. Similarly, (Zhang et al., 2012) prepared mixed matrix PES membrane using biogenic silver nanoparticles (bio $\mathrm{Ag}^{0}$ ) as additives. The surface of the nanocomposite membrane was tested to evaluate the attachment of $E$. coli and Pseudomonas aeruginosa (as pure cultures) and activated sludge (as a mixed culture). Results demonstrated that bio $\mathrm{Ag}^{0}$ addition showed excellent antibacterial activity, prevented bacterial attachment and reduced the biofilm formation on the membrane surface during nine weeks of monitoring, whereas the control membrane was heavily fouled. The diameter increment of the inhibition zone to P. aeruginosa was $16 \%, 20 \%, 24 \%$, and $30 \%$ for the $0.1,0.3,0.5,1 \mathrm{wt} \%$ silver content in the nanocomposite membrane. Furthermore, a gradual improvement in hydrophilicity was reported (from $66.6^{\circ}$ to $51.2^{\circ}$ ), with increasing the silver content in the matrix from 0 to $1 \mathrm{wt} \%$. (Kim et al., 2012) entrapped Ag-NPs in a thin film via interfacial polymerisation on a polyethersulfone PES substrate containing acid modified MWCNTs, high antibacterial activities were achieved and low adhesion of $P$. aeruginosa (PA01) to the membrane surface was observed. Results indicated that greater than $96 \%$ of attached bacteria on the n-TFN membrane but only less than $3 \%$ of attached bacteria on the n-TFC membrane were dead cells. Moreover, the thin-film nanocomposite (n-TFN) had better hydrophilicity and permeability compared to the original unmodified membrane while no change in roughness and rejection values for $\mathrm{NaCl}$ and $\mathrm{Na}_{2} \mathrm{SO}_{4}$ were observed before or after Ag-NPs addition. This suggests that Ag-NPs did not effectively influence the membrane surface characteristics: pore size and pore distribution. ( $\mathrm{Xu}$ et al., 2016b)blended HNS of Ag-coated $\mathrm{Cu}_{2} \mathrm{O}$ nanowires with PSF. L-dopa was grafted on the $\mathrm{Cu}_{2} \mathrm{O}$ surface to facilitate linking the AgNPs. The blended membranes exhibited outstanding bacteriostatic activity when tested against Escherichia coli and Staphylococcus aureus when the loading weight was $0.5 \mathrm{wt} \%$. ZOI diameter was $28.1 \mathrm{~mm}$ and $25.5 \mathrm{~mm}$ against E.coli and $S$. aureus, respectively.

\section{Copper-based nanocomposite membranes}

Antimicrobial activity is not limited to silver nanoparticles, the anti-biofouling properties of copper-based nanoparticles (Cu-NPs) are also well known. In addition to their antibacterial/antifungal applications, $\mathrm{Cu}-\mathrm{NPs}$ exhibit superior antioxidant, optical, 
catalytic and electrical properties that make them attractive for a broad range of applications (Akar et al., 2013; Huang et al., 2008). This includes antimicrobial agents in coating/paints (Acharya et al., 2004; De Kwaadsteniet et al., 2011), healthcare (O'Gorman \& Humphreys, 2012), energy storage (Jesumathy et al., 2011), chemical sensors (Zhang et al., 2011), catalysts (Kamal et al., 2013) and so on. Similar to Ag$\mathrm{NPs}$, the exact antimicrobial mechanism by which $\mathrm{Cu}-\mathrm{NPs}$ exhibit its biocidal effects is still unclear (O'Gorman \& Humphreys, 2012). (Ruparelia et al., 2008) speculated that they have a similar mechanism as Ag-NPs. (Bagchi et al., 2012) suggested that various mechanisms such as membrane disruption, complex formation with proteins, DNA damage and blocking of biochemical pathways were responsible for the antibacterial action of Cu-NPs. (Ramyadevi et al., 2012) claimed high inhibitory activity of copper nanoparticles against a diverse range of bacteria including Staphylococcus aureus, Klebsiella pneumonia, E. coli, P. aeruginosa and Micrococcus luteus, and fungi including Candida albicans, Aspergillus flavus and Aspergillus niger. However, incorporation of $\mathrm{Cu}$ in the synthesis and application of nanocomposite membranes has rarely been addressed.

Recently, (Isloor et al., 2013) conducted novel research to immobilise atomic copper onto PS/modified poly-isobutylene-alt-maleic anhydride blend membrane surface by a physical vapour deposition technique. The copper-coated membranes exhibited slightly higher salt rejection and surface roughness with only a slight decrease in pure water flux. Further characterisation to test the antimicrobial resistance of the coated membrane was investigated using the food poisoning organism Bacillus cereus. Good inhibition was observed against bacterial growth indicating that copper deposition could be an effective technique for the fabrication of antimicrobial membranes. (Akar et al., 2013) studied two types of nanoparticles; selenium (nSe) and copper $(\mathrm{nCu})$. $\mathrm{nSe}$ were prepared by the reduction of aqueous sodium selenite solution with freshly prepared glucose solution, while $\mathrm{nCu}$ were prepared by sonochemical reduction of copper(II) hydrazine carboxylate $\mathrm{Cu}-\left(\mathrm{N}_{2} \mathrm{H}_{3} \mathrm{COO}\right)_{2} 2 \mathrm{H}_{2} \mathrm{O}$ complex in an aqueous medium. The synthesised nanoparticles were incorporated at $(0.002,0.010$, 0.030 and 0.050 wt.\%) into UF/PES membrane via the classical phase inversion technique. The resultant nanocomposites were characterised with activated sludge as a biological suspension and bovine serum albumin (BSA) as a protein foulant model. The findings indicated good membrane antifouling properties against both activated sludge 
and BSA. Moreover, the morphology, hydrophilicity, and permeability of the nanocomposites were dependent on ENMs composition in the blend.

\section{Iron oxides-based nanocomposite membranes}

Iron is one of the most abundant metals in the earth's crust. Similar to other metals at the nanoscale level, iron nanoparticles, as a pure metal, are extremely reactive, which has made them difficult to study and restricted their practical applications ( $\mathrm{Ng}$ et al., 2013). For instance, zero-valent iron (nZVI) nanoparticles possess high reactivity and are easily oxidised to $\mathrm{Fe}^{+2}$ and/or $\mathrm{Fe}^{+3}$ ions when exposed to water (Tang \& Lo, 2013). However, iron compounds are relatively stable when present at the nanoscale level. Their crystallographic structures provide high surface area/volume ratio and superparamagnetic properties that offer a high reactivity. In addition to their excellent magnetic and reactivity, they have low toxicity, high surface modifiability, great biocompatibility and are chemically inert (Gupta \& Gupta, 2005; Xu et al., 2012). The unique features of iron oxide-based nanoparticles (Fe- NPs), mainly magnetite $\left(\mathrm{Fe}_{3} \mathrm{O}_{4}\right)$ and maghemite $\left(\gamma \mathrm{Fe}_{2} \mathrm{O}_{3}\right)$, have encouraged many researchers to investigate these engineered magnetic nanoparticles in remediation and water treatments. Depending on the oxidation state of the iron, these magnetic nanoparticles possess different chemical properties. Indeed, they offer distinct capabilities and reactivity for contaminants removal (Tang \& Lo, 2013). Based on the aforementioned unique characteristics, the addition of Fe-NPs is believed to impart a great potential to the fabrication of organomineral nanocomposite membrane with novel process control properties.

Impregnation of Fe-NPs into polymeric membranes is believed to introduce innovative solutions for many of the problems associated with polymeric membrane applications. In a recent study, (Homayoonfal et al., 2014) investigated the influence of iron oxide $\left(\mathrm{Fe}_{3} \mathrm{O}_{4}\right)$ nanoparticles immobilised in PSF membranes. The nanocomposites were synthesised via three techniques: deposition by interfacial polymerisation, deposition by photopolymerization and by blending into the polymeric matrix. These researchers concluded that the presence of $\mathrm{Fe}_{3} \mathrm{O}_{4}$ nanoparticles significantly enhanced membranes surfaces roughness, pure water flux, and surface hydrophilicity. The blending method performed better in terms of structural properties while deposition was better for dye separation yield. In another recent study, (Rambabu \& Velu, 2014) 
studied the influence of Fe-NP concentration on the resultant membrane composites. NPs were blended with PES at 0, 1, 2, 3 and 4wt.\% through the classical phase inversion method to fabricate Fe/PES-UF nanocomposite membrane. The results indicated that up to a certain Fe-NP concentration, synthesised membranes exhibited higher flux, thermal stability, and hydrophilicity than the original unmodified PES membrane. In addition, heavy metal ion $\left(\mathrm{Cu}^{+2}\right.$ and $\left.\mathrm{Zn}^{+2}\right)$ rejection was slightly decreased due to the increased pore size and porosity induced by the addition of nanoparticles. (Ghaemi et al., 2015) studied the influence of surface-modified $\mathrm{Fe}_{3} \mathrm{O}_{4}$ nanoparticles on $\mathrm{NF}$ membranes used for heavy metal removal. Fe-NPs modification was achieved by immobilisation of metformine, silica, and amine. Thereafter, modified Fe-NPs were blended in a PES/NF membrane matrix at various compositions. Similar to the previously mentioned studies, results indicated that Fe-modified nanoparticles influenced the morphology of the membranes with higher porosity and larger pore size found in all nanocomposites except that contained amine modified NPs. In addition, a further increase in surface roughness parameters, pure water flux and hydrophilicity were reported. However, the nanocomposite membrane prepared with 0.1 wt.\% metformine exhibited a higher rejection for copper ions (92\%) and higher membrane reusability due to a large number of $\mathrm{N}$ atoms around each particle which offered active adsorption sites through their lone electron pairs. In contrast to Ghaemi's findings, (Daraei et al., 2012) reported a decrease in pure water flux at all the different loadings of $0.01,0.1$ and $1 \mathrm{wt} \%$ of Fe-NPs and an increase in $\mathrm{Cu}(\mathrm{II})$ removal. (Alam et al., 2013) prepared $\mathrm{Fe}_{3} \mathrm{O}_{4} / \mathrm{PES}$ nanocomposite membrane for desalination applications. Their results showed higher rejection values (68\% and $82 \%$ ) for $\mathrm{NaCl}$ and $\mathrm{MgSO}_{4}$, respectively, at 10\% Fe-NPs loading. Furthermore, lower contact angle and smoother surfaces were obtained that showed promise for lower fouling properties.

\section{Aluminium oxide-based nanocomposite membranes}

Similar to other metal/metal oxide nanoparticles, $\mathrm{Al}_{2} \mathrm{O}_{3}$ has also attracted interest for many applications that include surface protective coating, catalysis, fire retardation and composite materials (Pathania et al., 2016). However, due to their high adsorption capacity, resistance to chemical agents and excellent catalytic performance for many reactions (Fouladgar et al., 2015; Wang et al., 2015b) $\mathrm{Al}_{2} \mathrm{O}_{3}-\mathrm{NPs}$ have gained the 
attention of membrane technologists for the development of new nanocomposite membranes with novel properties.

(Mehrnia et al., 2015) investigated the influence of alumina NPs on the morphology and performance of PSf nanocomposite membrane. $\mathrm{Al}_{2} \mathrm{O}_{3}-\mathrm{NPs}(70 \mathrm{~nm})$ were blended into PSf membrane via a classical phase inversion method at a concentration ranging from 0 to $0.52 \mathrm{wt} . \%$. The NPs loading threshold was found to be $0.39 \mathrm{wt} . \%$. Nanocomposite prepared at the loading threshold was further evaluated, and the characteristics of the resultant nanocomposite were compared up to and below the loading threshold by rheometric analysis, contact angle measurements and SEM images. By adding the NPs, rheological features of the casting solution changed from a Newtonian fluid to a non-Newtonian one. Hydrophilicity of the nanocomposites was enhanced by increasing the loading weight even after the threshold. Raising the concentration of NPs up to 0.39 wt.\% resulted in porosity development, flux enhancement and separation percentage reduction, while after this point, porosity diminished due to further increase in solution viscosity. In a similar study, (Homayoonfal et al., 2015) prepared $\mathrm{Al}_{2} \mathrm{O}_{3} / \mathrm{PES}$ nanocomposite membrane blend for membrane bioreactor applications. Two compositions were used (0.02 and $0.03 \mathrm{wt} . \%)$ in the preparation. The results suggested that the presence of alumina nanoparticles up to $0.03 \mathrm{wt} . \%$ in the mixed matrix membrane could hinder biofilm formation and provide a $75 \%$ reduction in cake layer resistance and up to $83 \%$ reduction in irreversible resistance. Thus, enhanced antifouling properties that were further confirmed by the reduced contact angle from 73 to 51. Furthermore, the pure water flux was more than four times that of the original PES membrane. (Ghaemi, 2016) investigated the ability of alumina NPs to improve adsorption of heavy metals and the removal efficiency of copper by PES membranes. Different amounts of alumina NPs $(0.01 \%, 0.1 \%$ and $1 \%)$ were blended to prepare the mixed matrix membrane. All membranes exhibited higher water permeability up to NPs loading $\leq 1 \mathrm{wt} . \%$ due to the enhanced porosity and hydrophilicity of the nanocomposites. In addition, larger microvoids in the support layer and thinner skin layer were also observed. Furthermore, copper removal efficiency was also improved. However, any further increase in $\mathrm{Al}_{2} \mathrm{O}_{3}-\mathrm{NPs}$ wt.\% did not show any change in the characteristics and performance of the nanocomposites. The authors suggested that the adsorption was the dominant separation mechanism in the nanocomposite membranes. In another study, a similar influence for alumina NPs on 
PES membrane morphology was observed, (María Arsuaga et al., 2013) compared the influence of three different metal oxide nanoparticles, $\mathrm{TiO}_{2}, \mathrm{ZrO}_{2}$ and $\mathrm{Al}_{2} \mathrm{O}_{3}$, on PES membrane. The nanocomposites fouling behaviour was characterised by using BSA and humic acid as model organic foulants. Their research demonstrated that $\mathrm{Al}_{2} \mathrm{O}_{3} / \mathrm{PES}$ nanocomposite membrane had higher pure water flux, higher rejection, long-term stability and lower flux decline for both model foulants in comparison to the other nanocomposites and original membranes. This behaviour was attributed to the fact that $\mathrm{Al}_{2} \mathrm{O}_{3}$ nanocomposite membrane has introduced more hydrophilic centres in the vicinity of the membrane surface, which reduced the possible adsorption of foulants. For MBR applications, (Maximous et al., 2009) prepared $\mathrm{Al}_{2} \mathrm{O}_{3}$ entrapped PES/UF membranes to characterise their fouling characteristics through activated sludge filtration. Nanocomposite membranes exhibited lower flux decline compared to the nascent membrane. In addition to a greater fouling mitigation with increasing $\mathrm{Al}_{2} \mathrm{O}_{3}$ content. In contrary with the previously mentioned literature, (Yan et al., 2005) found that the addition of $\mathrm{Al}_{2} \mathrm{O}_{3}$ NP did not show any influence on pore size and porosity of the nanocomposite membranes, while a noticeable enhancement was observed on surface hydrophilicity, permeation, antifouling and mechanical characteristics of the nanocomposites.

\section{Titanium dioxide-based nanocomposite membranes}

Titanium dioxide $\left(\mathrm{TiO}_{2}\right)$ is one of the most common materials in our daily life (Fujishima et al., 2000). It is estimated that of all the nanoparticles in consumer products, $\mathrm{TiO}_{2}$ nanoparticles $\left(\mathrm{TiO}_{2}-\mathrm{NPs}\right)$ currently have the highest degree of commercialisation (Vance et al., 2015). However, $\mathrm{TiO}_{2}-\mathrm{NPs}^{\prime}$ excellent hydrophilic and photocatalytic properties have led to attention in environmental purification applications (Fujishima et al., 2000). Under UV irradiation, $\mathrm{TiO}_{2}-\mathrm{NPs}_{\text {show a superior }}$ capability to deactivate microorganisms and to decompose organic compounds (Bai et al., 2015; Mills \& Le Hunte, 1997). Materials with photocatalytic properties could offer the possibility to introduce such functionalities when incorporated into polymeric membranes (Bergamasco et al., 2011).

According to Madaeni \& Ghaemi (2007), UV irradiation of $\mathrm{TiO}_{2}$ nanocomposite membranes could impart two functionalities to the membranes: photocatalytic and 
ultra-hydrophilicity. As a consequence of photocatalysis, groups of active oxidant reagents appear on the surface of the membrane which leads to decomposition and removal of the membrane foulants. While ultra-hydrophilicity could impart a selfcleaning property and increase the nanocomposite's water permeability as well. (Kim et al., 2003) prepared $\mathrm{TiO}_{2}$-NPs/TFC hybrid membrane via a self-assembly technique. The new nanocomposite membrane provided a substantial photo-bactericidal effect on E. coli under UV light irradiation. In another study, (Rahimpour et al., 2008) investigated the impact of incorporating $\mathrm{TiO}_{2}-\mathrm{NPs}$ on the antifouling properties of PES/UF membranes for application in the dairy industry. Three sets of membranes were fabricated: $\mathrm{TiO}_{2}$-bended membranes, UV-irradiated $\mathrm{TiO}_{2}$-blended membranes, and UV-irradiated $\mathrm{TiO}_{2}$-deposited membranes. $\mathrm{TiO}_{2}$ entrapped membranes showed lower milk water permeation and initial pure water flux in comparison to the original membrane. In contrast, however, flux stability and fouling resistance were better in the long term. On the other hand, UV irradiation of $\mathrm{TiO}_{2}$-blended membranes added further enhancement to the flux and antifouling properties whereas coating exhibited superior effects, compared to the other membranes, to mitigate membrane fouling and to overcome PES hydrophobicity. In another piece of research, (Li et al., 2009) prepared a novel $\mathrm{TiO}_{2}$ self-assembly nanocomposite membrane by immersing a blend membrane of poly(styrene-alt-maleic anhydride)/polyvinylidene fluoride (SMA/PVDF) in a suspension of $\mathrm{TiO}_{2}$. Their results demonstrated that permeability and antifouling properties against BSA were significantly improved when compared to the (SMA/PVDF) blend membrane. In another study, $\mathrm{TiO}_{2}$ NPs were used to fabricate a novel polyamide-titanium oxide $\left(\mathrm{PA}-\mathrm{TiO}_{2}\right)$ nanocomposite membrane via in situ interfacial polymerisation (Lee et al., 2008). Results demonstrated higher flux and hydrophilicity at 5 wt.\% $\mathrm{TiO}_{2}$, with high and stable rejection to $\mathrm{MgSO}_{4}$ (95\%). In another study, (Vatanpour et al., 2012) investigated the impact of nano- $\mathrm{TiO}_{2}$ type and size on the morphology, performance and antifouling properties of mixed matrix PES/NF membranes. Various types of $\mathrm{TiO}_{2}$ nanoparticles (PC500, PC105, and PC25) were used in the preparation. Pure water flux and hydrophilicity, for all the blended membranes, were higher compared to that of the original PES membrane. However, after a particular concentration of $\mathrm{TiO}_{2}$, nanoparticle aggregation occurred. This aggregation was more severe in the case of PC105 and PC500 membranes, and the increased concentration clogged the pores and reduced the pure water flux. 
Furthermore, the biofouling resulting from whey filtration tended to decrease with the smaller nanofiller size. More flux recovery was achieved due to the higher surface area and water adsorption affinity.

\subsubsection{Carbon-based nanomaterials}

I. Carbon nanotubes

Since the discovery of carbon nanotubes (CNTs) in 1991, CNTs have become an established material in commercial products (Balasubramanian \& Burghard, 2005) (Figure 2.10). A lot of interest and research endeavour has focused on CNTs due to their tuneable electrical and thermal characteristics, novel optical properties and superlative strength. Most of their applications to date have been focused within electronics, energy and composites sectors (Baughman et al., 2002). CNTs have been described as seamless cylinders derived from the honeycomb lattice of a graphite sheet (single atomic layer of crystalline graphite) (Imtani \& Jindal, 2006). They exist in three basic structures, namely, single-walled carbon nanotubes (SWCNTs), double-walled carbon nanotubes (DWCNTs) and multi-walled carbon nanotubes (MWCNTs). SWCNTs are made from a single-atom-thick sheet of graphene rolled up into a cylinder while DWCNTs have another graphene sheet around the central SWCNT. Likewise, MWCNTs are a series of concentric SWCNTs (Shaikjee \& Coville, 2012) (Figure 2.11). Due to their outstanding features, CNTs have been widely studied as nanofillers for the modification of polymeric membranes, mainly using MWCNTs and SWCNTs (Van der Bruggen, 2012). 

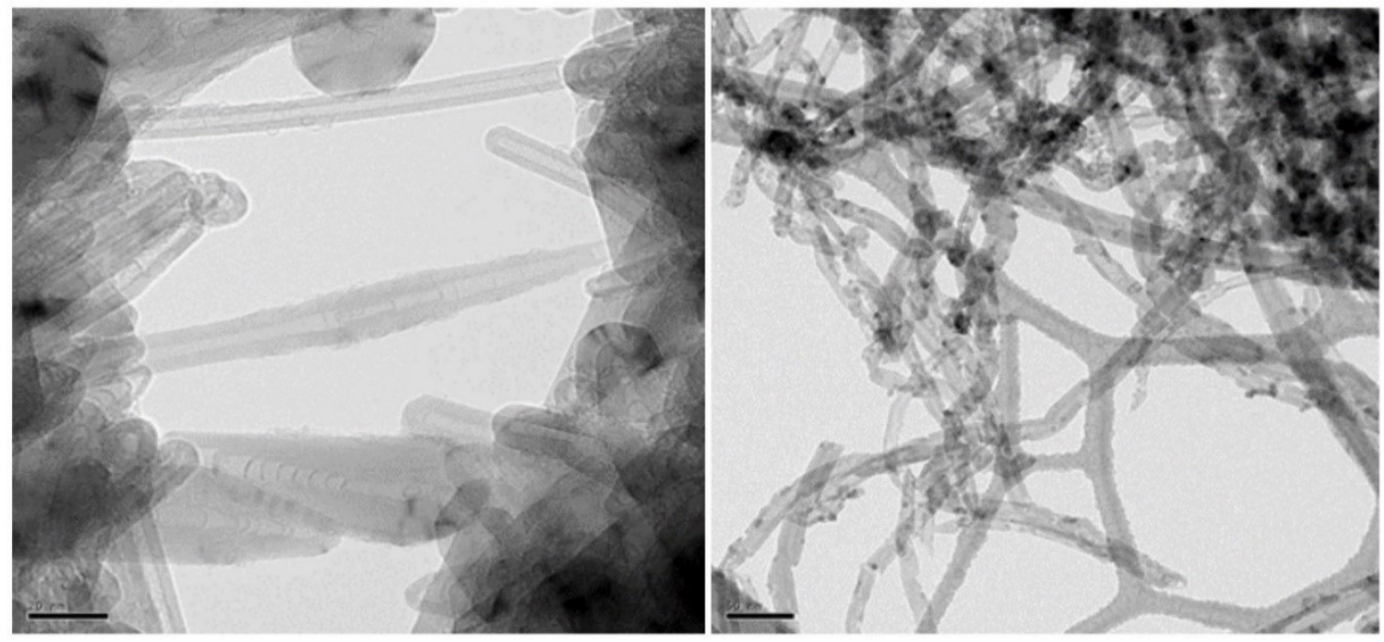

Figure 2.10: SEM images of CNTs. The left image presents CNTs (scale bar 20nm), the right image presents Fe-CNTs (scale bar 50nm).

The exact magnitude of CNTs properties relies mainly on the chirality, length, and diameter of the nanotubes themselves and whether they are MWCNTs, DWCNTs or SWCNTs (Sahoo et al., 2010; Xie et al., 2005). In a recent work, (Wang et al., 2015a) compared the performance of PES/NF mixed matrix membranes (MMMs) using two different diameters (20 and $40 \mathrm{~nm}$ ) of MWCNTs. Both MMMs showed higher salt rejection and water flux than the original PES membrane while MMMs embedded with thinner CNTs obtained better filtration performances than MMMs with thicker CNTs at 0.1 wt.\%. CNTs may act as water channels when impregnated with the membrane matrix. MWCNTs have been found to have great influence on permeation properties and the morphology of the membranes depending on the CNTs content (Celik et al., 2011b). In addition, MWCNTs can enhance the wettability of membrane materials and the electrostatic repulsion between the polymeric membranes and foulants (e.g. proteins) (Vatanpour et al., 2011). (Celik et al., 2011a) reported that MWCNTs/PES membrane had lower tendency to foul and smaller irreversible fouling ratio in comparison to the control PES membrane when using BSA and ovalbumin (OVA) for the fouling assessment. Furthermore, the fouled membranes could be more effectively treated by a water backwash cleaning process. In another study, (Shen et al., 2013) exploited MWCNs-grafted by poly(methyl methacrylate) (PMMA) to synthesize polyamide thin film composite (PA-TFC) membrane via interfacial polymerization, the results demonstrated higher rejection for $\mathrm{Na}_{2} \mathrm{SO}_{4}(99 \%)$ and the water flux was about 
$62 \%$ increased to that of the unmodified TFC membrane. In another study, (Kang et al., 2008a) observed a superior antimicrobial activity for SWCNTs, much higher than their MWCNTs counterparts., cell membrane damage is the most likely mechanism causing bacterial cell death on direct contact with SWCNTs.

CNTs are well known for their mechanical strength and they are the materials of choice for composites reinforcement (Coleman et al., 2006). (Shawky et al., 2011) claimed that the tensile strength and Young's modulus of the nanocomposite membrane could be increased with MWCNTs content addition. Similar findings were observed by (Chen et al., 2016) when (0-0.15 wt.\%) carboxyl functionalized MWCNTs were blended with PVDF/PVA UF membrane. At 0.12 wt.\% MWCNTs content in the matrix, the break strength, elongation at break and Young's modulus were enhanced by $60 \%, 215.5 \%$, and $56.7 \%$, respectively, in comparison to the original membrane.
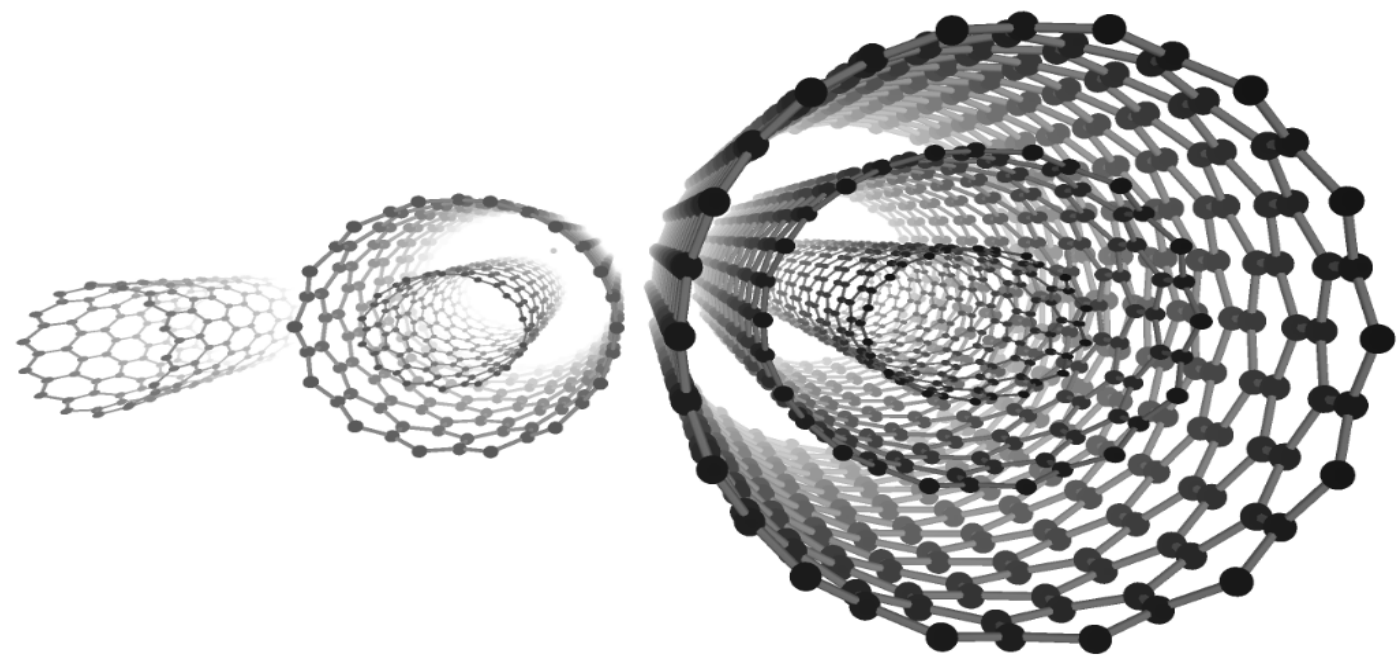

Figure 2.11: Arrangement of graphene sheets to produce SWCNTs, DWCNTs, and MWCNTs respectively (from left to right).

\section{Graphene and graphene oxide}

Graphene (G), has emerged as the "wonder" material of the $21^{\text {st }}$ century. With twodimensional monoatomic-thick building blocks of a carbon allotrope, graphene has better thermal, electrical and mechanical characteristics, higher aspect ratio and surface area than other materials including CNTs, Kevlar and carbon fibres (Singh et al., 2011), as shown in (Table 2.1). The significant potential of graphene has attracted enormous interest in applications for nanocomposites (Kuilla et al., 2010). The anticipated physicochemical properties of graphene-based polymer nanocomposites depend mainly 
on the interfacial bonding between the polymer matrix and graphene layers, in addition to the distribution level of graphene layers inside the polymer matrix. On the other hand, it should be noted that pristine graphene does not form homogeneous composites due to its incompatibility with organic polymers; this may create inferior composite properties (Bourlinos et al., 2003; Vickery et al., 2009). To overcome the polymer incompatibility issues of pristine graphene, oxidation of graphene has been suggested to introduce highly hydrophilic and stable colloidal dispersions (Geng et al., 2009). Graphene oxide (GO) is a highly chemically modified form of graphene that consists of a variety of functional groups, such as carboxylic acid at the edges and hydroxyl and epoxide groups on the basal plane. These functional groups can control the van der Waals forces and improve the compatibility with organic polymers to achieve better dispersion (Goenka et al., 2014). In recent years, incorporation of GO into polymeric membranes has gained more and more attraction. Various nanocomposite membranes have been fabricated to impart the exceptional characteristics of graphene into these membranes, like PSF-GO (Ganesh et al., 2013; Zhang et al., 2013b), PES-GO (Yu et al., 2013) and PVDF-GO (Xu et al., 2014).

Table 2.1: Properties of graphene, CNT, nano-sized steel and polymers (Kuilla et al., 2010).

\begin{tabular}{llll}
\hline Materials & $\begin{array}{l}\text { Tensile } \\
\text { strength }\end{array}$ & $\begin{array}{l}\text { Thermal conductivity (W/mk) at } \\
\text { room temperature }\end{array}$ & $\begin{array}{l}\text { Electrical } \\
\text { conductivity } \\
\text { (S/m) }\end{array}$ \\
Graphene & $130 \pm 10 \mathrm{GPa}$ & $(4.84 \pm 0.44) \times 103$ to $(5.30 \pm 0.48) \times 103$ & 7200 \\
CNT & $60-150 \mathrm{GPa}$ & 3500 & $3000-4000$ \\
Nano-sized steel & $1769 \mathrm{MPa}$ & $5-6$ & $1.35 \times 106$ \\
Plastic (HDPE) & $18-20 \mathrm{MPa}$ & $0.46-0.52$ & Insulator \\
Rubber (natural rubber) & $20-30 \mathrm{MPa}$ & $0.13-0.142$ & Insulator \\
Fiber (Kevlar) & $3620 \mathrm{MPa}$ & 0.04 & Insulator \\
\hline
\end{tabular}

According to Chang et al. (2014), graphene oxide has demonstrated high hydrophilicity and anti-fouling properties against BSA when incorporated within $\mathrm{PVDF} / \mathrm{UF}$ membrane, this is attributed to the electrostatic repulsion of GO, which acts as a barrier to prevent BSA adsorption on the nanocomposite membrane surface. (Zinadini et al., 2014) reported that carboxylic acid, hydroxyl and other functional groups of GO surfaces are migrated to the surface of the membrane during the phase 
separation fabrication process and thus enhanced the surface properties and hydrophilicity of the nanocomposite membrane. Similarly, these enhanced interactions and hydrophilicity were also reported in membrane bioreactor (MBR) applications using GO-PSF MMMs (Lee et al., 2013). In addition, GO was also found to have antibacterial activities (Akhavan \& Ghaderi, 2010). The presence of functional groups ensures high negative zeta potential and impedes attachment of biofouling and subsequent accumulation on the membrane surface (Liu et al., 2010). (Hu et al., 2010) reported high toxicity to $S$. aureus and $E$. coli, which was mainly attributed to the mechanical damage of the bacterial cell membrane caused by the sharp edges of GO as revealed by a study using transmission electron microscopy TEM. In another study, (Yu et al., 2013) found superior anti-biofouling properties for GO nanosheets modified by hyperbranched polyethyleneimine (HPEI) when blended with PES/UF membrane.

From the mechanical performance perspective, in pressure-driven membrane processes, high tensile strength and elongation at break values mean better toughness and sustained the integrity of membranes, which will assure a comprehensive performance and provide good abilities for repeated use and long-term membrane separation applications (Zhang et al., 2013d). Graphene and its derivatives have been widely used for composite reinforcement purposes as graphene is the stiffest and strongest material known (Cano et al., 2013). It has been reported that the enhancement in the mechanical properties of graphene-polymer composites is much higher than that of clay or other carbon-based polymer nanocomposites (Quan et al., 2009). The enhancements of graphene-based nanocomposites can be achieved at very low filler content in the polymer matrix (Young et al., 2012). A comparative study was carried out by (Zhang et al., 2013a), who studied the impact of blending OMWCNTs, GO and OMWCNTs-GO on the mechanical properties of PVDF ultrafiltration membrane fabricated via a phase inversion technique. These researchers reported superior enhancement in tensile strength, $12.86 \%$, and $43.94 \%$, and elongation at break, $31.50 \%$, and $39.24 \%$, for the OMWCNTs and GO synthesised composite membranes, respectively. The slightly less mechanical performance was shown for the MWCNTsGO/PVDF membrane due to the bigger pore size that stemmed from the synergetic effect for the oxidised low-dimensional carbon nanomaterials. (Xu et al., 2014) studied the influence of GO functionalization with 3-aminopropyltriethoxysilane (APTS) on the mechanical properties of PVDF/UF membrane. The tensile strength measurement 
of the $\mathrm{f}-\mathrm{GO} / \mathrm{PVDF}$ membrane was improved by $69 \%$ while the elongation at break was $48 \%$ more in comparison to GO/PVDF. Thus, GO could be a promising solution to fabricate hybrid membranes with excellent reinforced, antifouling and permeation properties.

\subsubsection{Cellulose-based nanoscale materials}

Recent interest has been applied to produce so-called green or eco-composite materials. Such eco-composites include nanocellulose based materials, which have been widely used as reinforcement materials due to their sustainability, green and environmentally friendly specifications, (Abdul Khalil et al., 2012; Ganster \& Fink, 2006; Henriksson \& Berglund, 2007). Nanocellulose applications to hybrid composite materials (Figure 2.12) have shown promising results for the three categories of nanocellulose: nanofibrillated cellulose (NFC), cellulose nanocrystal (CNC) and bacterial nanocellulose (BNC) (Abdul Khalil et al., 2014). Interestingly, these materials not only possess the properties of natural cellulose, which include hydrophilicity, biodegradability, and renewability but also has the characteristics of nanomaterials, for instance, high mechanical strength, high tensile modulus and high specific surface area (Klemm, 2006). However, there are only a few examples of nanocomposite membrane applications.
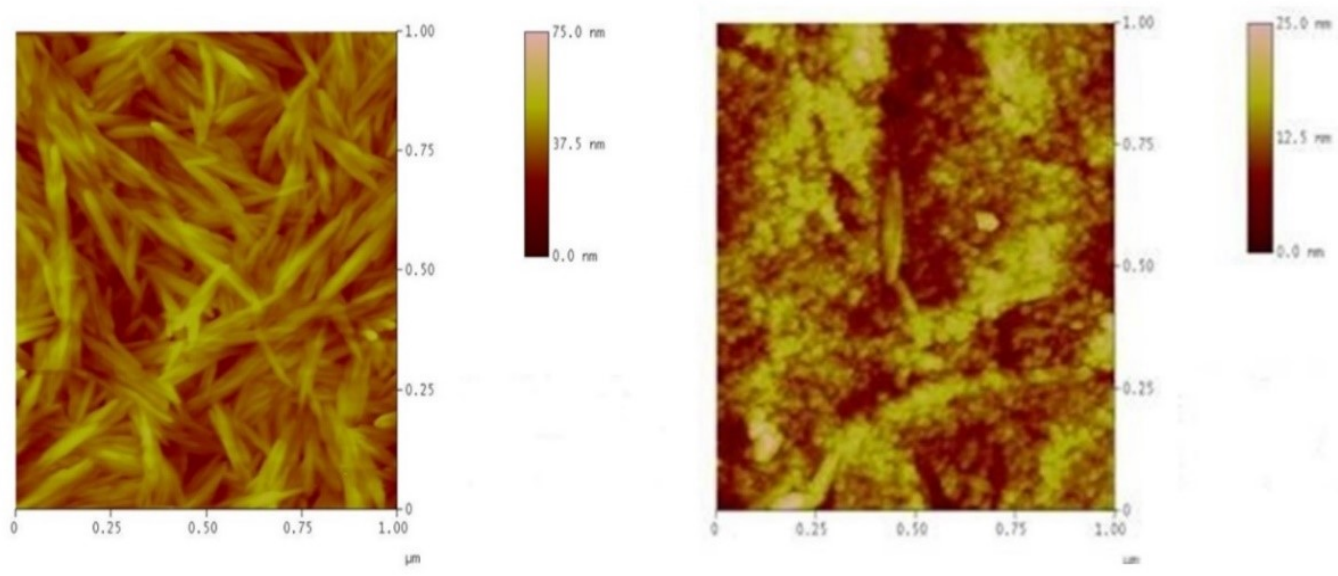

Figure 2.12: AFM images of dried cellulose nanocrystals (left), and fabricated membrane impregnated with $4 \%$ cellulose nanocrystals (right). Adopted from (AlMalek, 2012). 
(Bai et al., 2012) prepared CNC/PVDF mixed matrix membrane to investigate the influence of $\mathrm{CNC}$ composition (0.05-0.25 wt.\%) on the nanocomposite performance. Their results demonstrated that $\mathrm{CNC}$ has great influence on the surface characteristics and morphology of the nanocomposites. For CNC content up to $0.1 \%$, pure water flux, porosity and mean pore size increased without significant change in BSA rejection. In addition, both elongation-at-break and the tensile strength of the nanocomposite improved as well (from $84 \%$ to $150 \%$ ) and (from 4.3 to $6.3 \mathrm{MPa}$ ), respectively. Similarly, (Al-Malek, 2012) blended higher concentrations (2, 4, 6 wt.\%) of CNC into PES membrane. Increasing the CNC content in the casting solution was found to increase pore size, pore size distribution and pure water flux of the nanocomposites, while no impact was observed on surface roughness parameters. Moreover, the nanocomposite membrane with 2 wt.\% CNC exhibited higher strength (8.843 $\mathrm{MPa})$ with elongation of $6 \%$ in comparison to the control membrane (4.186 MPa), this was attributed to the structural changes in the membranes in the presence of $\mathrm{CNC}$ that changed the macrovoids to a more homogeneous and narrow form. However, the further increase in $\mathrm{CNC}$ content decreased the strength of the membrane to 8.047 and 4.63 $\mathrm{MPa}$ for the 4 and $6 \mathrm{wt} . \%$, respectively, which are still higher than that of the unmodified membrane. In another work, NFC/PES nanocomposites were prepared at different NFC loading weights. In comparison with pure PES membrane, the mean pore size, porosity, pure water flux, mechanical strength and elongation at break of nanocomposites were the highest at $1 \mathrm{wt} . \%$. However, the further increase resulted in a decrease in all characteristics ( $\mathrm{Li}$ et al., 2011). These findings suggest a threshold content of cellulose-based materials in composites, where optimum exploitation can be achieved.

Based on the previously mentioned sections, a conclusion can be drawn that ENMs could have distinguished influence on polymeric membranes depending on their dimension and type, as summarised in Table 2.2.

Table 2.2: Influence of various types of ENMs on polymeric membranes

\begin{tabular}{|c|c|c|c|c|}
\hline $\begin{array}{l}\text { Type of } \\
\text { membrane }\end{array}$ & Foulant & $\begin{array}{l}\text { Type of } \\
\text { ENMs }\end{array}$ & Influence on modified membranes & Ref. \\
\hline $\mathrm{UF} / \mathrm{PS}$ & $\begin{array}{l}\text { Pseudomonas } \\
\text { Mendocino } \\
\text { (KR1) } \\
\text { E. coli }(\mathrm{K} 12)\end{array}$ & $\mathrm{Ag} \mathrm{NP}$ & $\begin{array}{l}\text { - Improved hydrophilicity. } \\
\text { - No effect on membranes surface } \\
\text { charge and morphology. }\end{array}$ & $\begin{array}{l}\text { (Zodrow et } \\
\text { al., 2009) }\end{array}$ \\
\hline
\end{tabular}




\begin{tabular}{|c|c|c|c|c|}
\hline & Virus & & $\begin{array}{l}\text { - A slight increase in pure water } \\
\text { permeability. } \\
\text { - Enhance antibacterial and virus } \\
\text { removal, inhabited biofilm growth. }\end{array}$ & \\
\hline NF/PES & $\begin{array}{l}\text { P. aeruginosa } \\
\text { (PA01) }\end{array}$ & Ag NP & $\begin{array}{l}\text { - Enhanced antibacterial, } \\
\text { hydrophilicity and permeability. } \\
\text { - No effect on surface roughness, and } \\
\text { salt rejection. }\end{array}$ & $\begin{array}{l}\text { (Kim et al., } \\
\text { 2012) }\end{array}$ \\
\hline UF/PES & $\begin{array}{l}\text { Activated } \\
\text { sludge } \\
\text { BSA }\end{array}$ & $\mathrm{Cu} \mathrm{NP}$ & $\begin{array}{l}\text { - Decreased permeability. } \\
\text { - Increased hydrophilicity and protein } \\
\text { rejection }\end{array}$ & $\begin{array}{l}\text { (Akar et al., } \\
\text { 2013) }\end{array}$ \\
\hline UF/PES & Dye & $\mathrm{Fe}_{2} \mathrm{O}_{3}$ & $\begin{array}{l}\text { - Improved hydrophilicity, surface } \\
\text { roughness, dye removal and } \\
\text { permeability }\end{array}$ & $\begin{array}{l}\text { (Homayoonfal } \\
\text { et al., 2014) }\end{array}$ \\
\hline UF/PES & $\begin{array}{l}\text { Heavy metals } \\
\left(\mathrm{Cu}^{+2} \text { and } \mathrm{Zn}^{+2}\right)\end{array}$ & $\mathrm{Fe}_{3} \mathrm{O}_{4}$ & $\begin{array}{l}\text { - Enhanced hydrophilicity, water } \\
\text { permeability, thermal stability. } \\
\text { - Slight decrease in heavy metals } \\
\text { rejection } \\
\text { - Increased pore size and porosity. }\end{array}$ & $\begin{array}{l}\text { (Ghaemi et } \\
\text { al., 2015; } \\
\text { Rambabu \& } \\
\text { Velu, 2014) }\end{array}$ \\
\hline UF/PES & $\begin{array}{l}\text { BSA } \\
\text { Humic acid }\end{array}$ & $\mathrm{Al}_{2} \mathrm{O}_{3}$ & $\begin{array}{l}\text { - Higher water permeability, } \\
\text { hydrophilicity, rejection, and long- } \\
\text { term stability. } \\
\text { - Lower flux decline compared to the } \\
\text { neat membrane. }\end{array}$ & $\begin{array}{l}\text { (María } \\
\text { Arsuaga et al. } \\
\text { 2013) }\end{array}$ \\
\hline NF/PES & Whey & $\mathrm{TiO}_{2}$ & $\begin{array}{l}\text { - Improved permeability and } \\
\text { hydrophilicity } \\
\text { - Improved antifouling characteristics } \\
\text { and flux recovery. }\end{array}$ & $\begin{array}{l}\text { (Vatanpour et } \\
\text { al., 2012) }\end{array}$ \\
\hline $\mathrm{RO} / \mathrm{PVA}$ & Whey & $\mathrm{TiO}_{2}$ & $\begin{array}{l}\text { - Enhanced water permeability and } \\
\text { self-cleaning property (reduced } \\
\text { fouling and increased whey flux). } \\
\text { - Enhanced hydrophilicity and } \\
\text { photocatalytic characteristics. }\end{array}$ & $\begin{array}{l}\text { (Madaeni \& } \\
\text { Ghaemi, } \\
\text { 2007) }\end{array}$ \\
\hline $\mathrm{RO} / \mathrm{PA}$ & $\begin{array}{l}\mathrm{Ca}\left(\mathrm{HCO}_{3}\right)_{2} \\
\mathrm{BSA}\end{array}$ & MWCNTs & $\begin{array}{l}\text { - Enhance membrane surface charge. } \\
\text { - Affected the morphology. } \\
\text { - Increased water flux and a slight } \\
\text { decrease in rejection. } \\
\text { - Better antifouling and antioxidative } \\
\text { properties. } \\
\text { - }\end{array}$ & $\begin{array}{l}\text { (Zhao et al., } \\
\text { 2014) }\end{array}$ \\
\hline UF/PVDF & BSA & GO & $\begin{array}{l}\text { - The improved mechanical strength } \\
\text { of the modified membrane. } \\
\text { - Enhance, hydrophilicity, antifouling } \\
\text { and permeation properties. }\end{array}$ & $\begin{array}{l}\text { (Xu et al., } \\
\text { 2014) }\end{array}$ \\
\hline
\end{tabular}




\subsubsection{Issues arising from the incorporation of ENMs into polymeric membranes}

Despite the huge attention and funding devoted so far to the applications of ENMs in membrane technologies, the commercialisation of ENMs-membrane composites has yet to be achieved. In fact, with the marked demand of nanomaterials in the marketplace, there is a growing public debate whether the social and environmental cost of nanomaterials outweighs their benefits (Colvin, 2003). Apart from this debate, ENM's durability under various operating conditions as well as their degradability at the end of their service life represents the main challenges that have not yet been fully addressed (Kim \& Van der Bruggen, 2010; Kumar et al., 2009). In polymeric membrane applications, incorporation of ENMs also has some obstacles. Predominantly, their limited dispersion in the casting solutions, especially for nanomaterials having a diameter $<100 \mathrm{~nm}$ ( $\mathrm{Ng}$ et al., 2013). Poor dispersion results in nanomaterial agglomeration and uneven distribution in the polymeric matrix. This agglomeration may give rise to undesired changes in the nanocomposite membrane characteristics such as free surface energy, pore size, hydrophilicity, roughness and antifouling properties (Razmjou et al., 2011). Weak interfacial interaction/adhesion between ENMs and the polymeric structure will end up in the leaching of these ENMs out of nanocomposite membranes, causing a gradual deterioration in the nanocomposite membrane over time, reducing performance stability and potentially raising many environmental issues (Dong et al., 2012). These weak interactions have been mainly attributed to poor polymer-inorganic incompatibility, poor polymer-particle adhesion, the different thermal expansion coefficients for the ENMs and the polymer and solvent evaporation during nanocomposite formation (Hilal et al., 2012). In addition, agglomeration could be induced from incorporating a high content of ENMs during the fabrication (Gilbert et al., 2009). From an environmental point of view, there is a growing concern regarding the potential hazards of ENMs release into ecosystems. This is compounded by the lack of quantitative data available from monitoring their release, or knowledge as to which form they have been released into the environment (Gottschalk \& Nowack, 2011). For instance, De Kwaadsteniet et al. (2011) reported that Ag-NPs can form a composite colloid with some organics leading to entirely different toxicity from that of pure Ag nanoparticles. Undoubtedly, the potential hazard posed by ENMs necessitates the understanding and evaluation of the nature, effects, and detection of the ENMs toxicity. Even though the long-term health effects of ENMs 
is not adequately determined yet, there is an increasing evidence supporting that these nanomaterials have genotoxic and carcinogenic potential with commercial applications (Doak et al., 2017). Doak and coworkers are one of the pioneered research groups who extensively investigated and assessed the damage to the genetic material in living systems caused by various ENMs (Singh et al., 2017). Therefore, the environmental studies should not only quantify the released nanoparticles but also assess the toxicity of released nanoparticle composites. Very little is known regarding the transport and fate of ENMs in environmental waters since the bulk of the current nanotoxicological research is laboratory scale studies focusing on single species in a simple media (Bernhardt et al., 2010). Thus, ensuring the reliability of nanocomposites through minimising their toxicity in biotic and abiotic environments could offer new prospects regarding their industrialisation.

\subsubsection{Future improvements for ENMs incorporation into polymeric membranes}

One of the pragmatic approaches used to improve the interactions between the inorganic ENMs and the organic polymer chains is by revising the surface characteristics of ENMs. Several methods have been suggested for alteration of these characteristics through introducing a specific functional group at their surfaces. This research strategy is required to achieve improved interactions and more homogenous distribution for the ENMs within the polymeric matrix. Such methods include chemical treatments, grafting of synthetic polymers, ligand exchange techniques and adsorption of polymeric dispersants (Kango et al., 2013). Among these techniques, surface functionalization/modification by chemical treatment is currently an intensive research focus in nanocomposite membrane applications.

Surface functionalization/modification increases the stability of ENMs in their host materials, and various functional groups can be used to achieve this, such as carboxylic acid, phosphoric acid, silane coupling agents and dopamine (Figure 2.13) (Dias et al., 2011). Silanes have been recently applied as coupling agents to modify ENM surfaces and promote adhesion in the fabrication of nanocomposite membranes. Silanes are bifunctional molecules that possess dual reactivity, that enables them to act as intermediate materials to link two dissimilar materials (Mittal, 2007; Plueddemann, 2013). Polysiloxane has been used as a saline coupling agent, to chemically functionalize $\mathrm{SiO}_{2}$ nanoparticles and then to immobilise PEG molecules on their 
surface (Wu et al., 2013). The modified nanoparticles were then dispersed in a casting solution to prepare modified $\mathrm{SiO}_{2} / \mathrm{PVDF}$ nanocomposite membranes. The stability of the chemically modified $\mathrm{SiO}_{2}$ nanoparticles in the membrane matrix was significantly improved during membrane filtration processes and membrane cleaning, enabling the long-term usage of the blend membrane. A similar study reported good dispersion for chemically modified $\mathrm{TiO}_{2}$ in PES nanocomposite membrane when $\mathrm{TiO}_{2}$ particles were first modified using aminopropyltriethoxysilane (APTES) as a silane coupling agent (Razmjou et al., 2011). In a similar study, a novel PA/TFN nanofiltration membrane was prepared by dispersing aminosilanized $\mathrm{TiO}_{2}$ nanoparticles into a diamine monomer solution followed by polymerisation (Rajaeian et al., 2013). The silane coupling agent was grafted onto the surface of $\mathrm{TiO}_{2}$ to avoid agglomeration encouraged during the TFN preparation and to obtain a greater interfacial adhesion between the polymer matrix and $\mathrm{TiO}_{2}$ nanoparticles. Functionalized graphene oxide (f-GO) nanosheets with 3-aminopropyltriethoxysilane (APTS) were found to have better dispersion in organic solvents that non-functionalized GO (Xu et al., 2014). Polydopamine (PDA), also known as "bio-glue", has been recently used as an excellent coupling ligand in nanocomposite membranes. (Zhang et al., 2013c) conducted research to robustly bind $\mathrm{TiO}_{2}$ nanoparticles on the surface of TFC membrane using PDA. PDA can be selfpolymerised on $\mathrm{TiO}_{2}$ and membrane surfaces forming a firm connection between them. Similarly, $\mathrm{TiO}_{2}$ nanoparticles have been strongly bound and homogeneously distributed into PVDF ultrafiltration membrane by exploiting PDA (Shao et al., 2014). (Zhao et al., 2016) uniformly embedded PDA modified MWCNTs (PDA-MWCNTs) in polyamide (PA) thin-film composite membranes. Their results demonstrated a fine dispersion for the coated MWCNTs in polyethyleneimine aqueous solutions, which was interracially polymerised with trimesoylchloride $\mathrm{n}$-hexane solutions to fabricate nanocomposite membranes. The improved compatibility/interactions between the modified MWCNTs and PA matrix were attributed to the PDA coating layer on the nanotubes surface. In another study, (Khalid et al., 2015) prepared functionalized MWCNTs with dodecylamine (DDA) that were then used to fabricate PS nanocomposite membrane for desalination applications. The long alkyl chains of DDA functionalized MWNTs were found to improve the interfacial interactions/adhesion and compatibility between inorganic nanotubes and the polymeric matrix. 


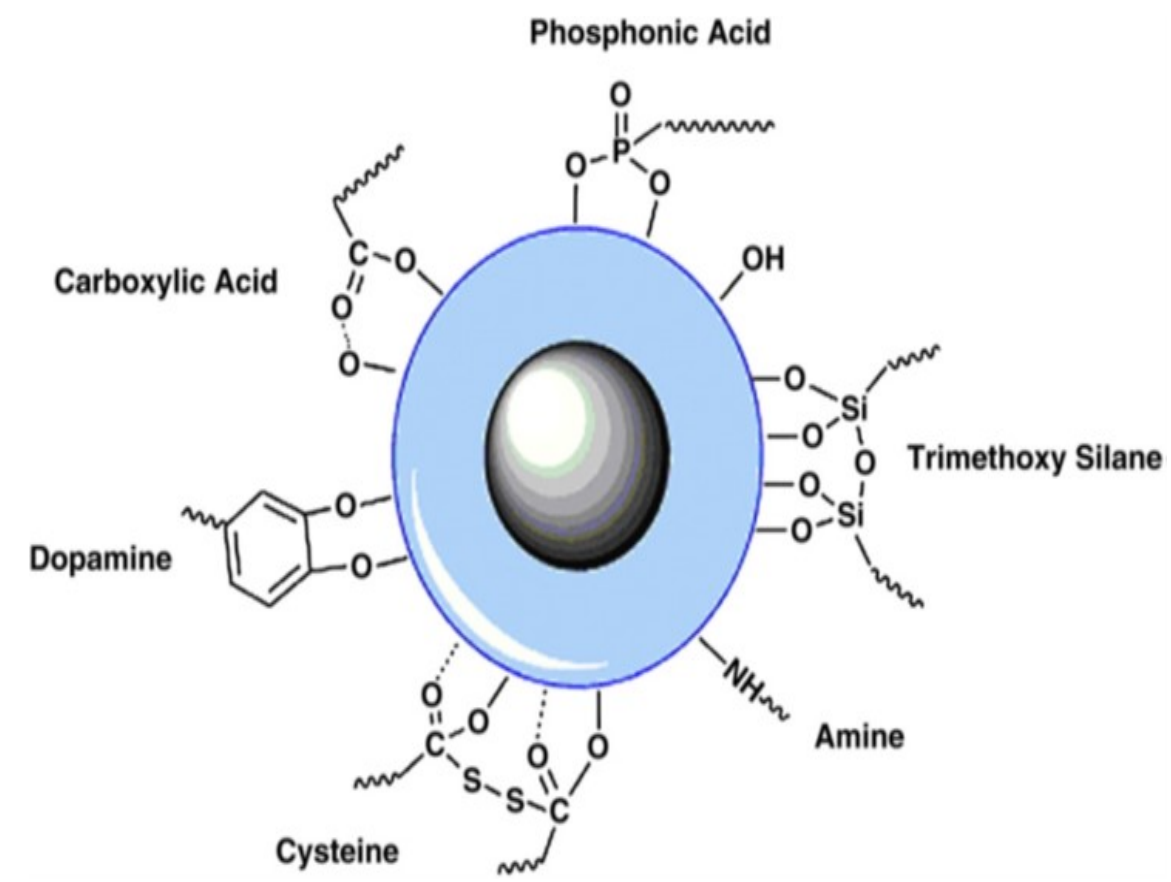

Figure 2.13: Different functional groups attached to iron oxide NPs surface (Dias et al., 2011).

\subsection{Conclusions}

The diverse and often unique properties of ENMs provide the membrane technologist with an extended toolbox for the bespoke fabrication of nanocomposite membranes with properties ideally suited for a particular process. ENMs have the potential for the creation of membranes that are optimised to meet all the challenges of desalination and wastewater treatment including fouling and biofouling while extending the lifetime of the membrane by enhancing their mechanical robustness and resistance to cleaning regimes. All of this with no impact on selectivity. An impressive list indeed of the benefits for membrane processes but arguably highly achievable. Literature has witnessed tremendous advances in all aspects of life as a result of the improved capabilities in the monitoring, control, and fabrication of materials at the nanoscale, these advances are beginning to impact on the quality and functionality of membrane materials used in water treatment. The dissemination of nanotechnological experience is set to continue and will inevitability impact positively on the engineering of membrane processes. However, despite of the advance state of nanocomposite 
membranes application at the lab scale level, nanocomposite membranes have not commercialised yet, where an argument about their environment and social costs has been raised recently. Herein, the following work presented aims to resolve several of the deficiencies reported in the literature for the applications of nanocomposite membranes. Despite of the relatively big amount of research papers on nanocomposite membranes, several gaps can be noticed in the literature and summarised as follows:

- The majority of current researchers are focussing on developing new nanocomposite membranes aiming for enhancing the surface free energy of the membrane's surface in the short term only. Although this is the main objective for polymeric modification through using nanoscale materials, but this should be done with anticipating and referring to the consequences arise from incorporation these nanomaterials, over the prolonged operation, on performance and surface characteristics of nanocomposites.

- Weak interfacial interactions between nanoscale materials and the polymeric matrix are the main bottlenecks in the applications of nanocomposite membranes. In the literature, there is lack of systematic studies on the enhancing and evaluation of adhesion/compatibility between NMs and polymeric matrix in order to extend the nanocomposite membrane lifespan.

- Regarding the high environmental and social costs of the nanocomposite membranes application as a result of the rapid depletion of 0D-NMs into the ecosystem. Only few research are focusing on $0 \mathrm{D} / 1 \mathrm{D}$ or $0 \mathrm{D} / 2 \mathrm{D}$ hybrid nanostructures in membrane separation applications, as a replacement for 0D-NMs in order to minimise NMs leach out, while there is no research on the application of these HNS in TFN membrane to resolve these deficiencies.

- Lack of efficient and reliable methodologies to incorporate higher amounts of NMs for TFN membrane applications. Most of TFN membranes incorporate NMs by either dispersion in the liquid or organic phase where only a small amount can be incorporated with uneven distribution in the PA matrix.

- Rapid depletion of the antibacterial activity of nanocomposite membranes fabricated by impregnation of $0 \mathrm{D}$ silver nanoparticles. A considerable 
amount of both surface-modified and unmodified AgNPs will be available at the top surface of the nanocomposite membranes. Presenting these NPs at the interface will expose them to the shear of water current. As a consequence, induce their leaching and diminish the antibacterial activity of the nanocomposite over time.

This thesis attempts to apply novel HNS comprise M/MO NPs coated MWCNTs for membranes modification purposes, instead of using M/MO NPs directly. MWCNTs with 2D structure will be employed as a carrier to tether the 0D NPs on their surfaces. This will be carried hand by hand with further improvement of the adhesion/compatibility between the HNS and polymeric matrix in order to avoid the bottlenecks of nanocomposite membrane applications. Ultimately, the new entity of nanocomposites can manifest enhanced hydrophilicity, permeability, thermal and chemical stability, porosity and mechanical properties along with greater stability within the polymeric matrix.

These overall objectives will be achieved by:

- Improving the design and fabrication of nanocomposite membranes to overcome the limitations of their applications through improving the incorporation protocol that ideally concentrates the NMs on or/and close to the selective layer of the membranes in order to extend the function of nanocomposite and their lifespan.

- Introducing ENMs into polymeric membranes and identifying how the characteristics of the ENMs, such as the high surface area to volume ratio and mechanical strength, can be used to optimise and tailor membranes for particular applications.

- Developing facile approaches for HNS fabrication aiming for tethering metallic nanoparticles robustly on the surface of CNTs templates via facile, rapid, scalable and cost-effective routes, and targeting better stabilisation and dispersion of 0D-nanomaterials.

- Reducing the environmental and economic costs through avoiding the NMs wastage during the TFN preparation by avoiding the limitations of conventional TFN fabrication techniques. 
- Creating a stable and defect free TFN membranes through surface modification of HNS in order to improve the compatibility between HNS and the PA thin layer.

- Improving the surface free energy of nanocomposite membranes surface to diminish the fouling along with other characteristics e.g., selectivity, chemical stability, porosity, permeability, thermal and mechanical properties. 


\section{Chapter Three}

\section{Materials and Methods}

Materials, analytical, support equipment, and experimental procedures used in this work are described in this chapter. The first section listed all materials being used for polymeric membranes and hybrid nanostructure fabrication, while the second has covered the essential and support equipment for the characterisation processes. In the last section, experimental procedures of all fabrication and filtration protocols were illustrated in detail.

\subsection{Materials}

Note: all chemicals used in this work were used as purchased without further purification, except nanotubes. And the ultra-pure water, used in all experiments, was produced by Milli - Q system supplied by Millipore and has $18.2 \mathrm{M} \Omega . \mathrm{cm}$ resistivity.

\subsubsection{Materials for membrane fabrication}

Polyethersulfone (PES) was chosen as the host polymer for membranes fabrication due to its excellent resistance to chemicals and chlorine attack, good thermal and mechanical properties, and applicability for a wide range of $\mathrm{pH}$ (Qin et al., 2005; Sathish Kumar et al., 2015). PES is well known as excellent membrane formation material for MF, UF and even NF, and as a support substrate for NF and RO membranes. The technical grade PES flakes - Ultrason ${ }^{\circledR}$ E 6020 P (m.wt=75,000 $\mathrm{g} / \mathrm{mol}$ ), as a versatile material for the production of tailor-made membranes, were kindly donated by BASF Co. Ltd (Germany).

Polyvinylpyrrolidone (PVP $40 \mathrm{~K})$ with a molecular weight of $(40,000 \mathrm{~g} / \mathrm{mol})$ was used as pore former and to enhance the interconnectivity of membranes structure. $\mathrm{N}$ - 
methyl-pyrrolidone (NMP), anhydrous with purity $99.5 \%$, has been used as a solvent for membrane fabrication due to its high stability, polarity, chemical resistance and boiling point $\left(192 \mathrm{C}^{\circ}\right)$. Chemicals used for TFN fabrication; $n$-Hexane (purity $\geq 95 \%$ ), m-Phenylenediamine (MPD), 1,3,5-Benzenetricarbonyl trichloride (TMC) were all purchased from Sigma Aldrich, UK.

\subsubsection{Materials for hybrid nanostructures fabrication}

Raw MWCNTs, produced by chemical vapour deposition (CVD) method, were purchased from Chengdu Organic Chemicals Co. Ltd. (China). Specifications of the nanotubes are given in Table 3.1. Nitric acid $\left(\mathrm{HNO}_{3}\right) 69 \% \mathrm{wt} / \mathrm{v}$, iron (III) nitrate nonahydrate $\left(\mathrm{Fe}\left(\mathrm{NO}_{3}\right)_{3} .9 \mathrm{H}_{2} \mathrm{O}\right)$, silver nitrate $\left(\mathrm{AgNO}_{3}\right)$, aluminium-nitrate nonahydrate $\left(\mathrm{Al}\left(\mathrm{NO}_{3}\right)_{3} .9 \mathrm{H}_{2} \mathrm{O}\right)$, titanium (IV) butoxide $\left(\mathrm{Ti}\left(\mathrm{OCH}_{2} \mathrm{CH}_{2} \mathrm{CH}_{2} \mathrm{CH}_{3}\right)_{4}\right)$, absolute methanol and ethanol, Trizma-base and dopamine hydrochloride were purchased from Sigma Aldrich, UK.

Table 3.1: Specification of the MWCNTs

\begin{tabular}{ll} 
MWCNTs specifications & Description \\
\hline Purity & $>95 \%$ \\
ID & $5-10 \mathrm{~nm}$ \\
OD & $10-20 \mathrm{~nm}$ \\
SSA & $>200 \mathrm{~m}^{2} / \mathrm{g}$ \\
Length & $0.5-2 \mu \mathrm{m}$ \\
Ash & $<1.5 \mathrm{wt} . \%$ \\
True density & $2.1 \mathrm{~g} / \mathrm{cm}^{3}$ \\
\hline
\end{tabular}

\subsubsection{Materials for characterisation and fouling tests}

Various molecular weights $(600,1150,3400,6000,8000,10000,20000$ and 35000) of Polyethylene (PEG) in addition to a larger molecular weight of Polyethylene oxide (PEO) 100000 were used as uncharged organic models to determine the pore size and pore size distribution of the formatted membranes. Humic acid (HA, $\sim 2-500 \mathrm{kDa}$ ) (CAS. No. 1415-93-6) as natural organic matter, Sodium Alginate (NaAlg, 12-80 $\mathrm{kDa}$ ) from Brown algae (CAS. No. 9005-38-3) as polysaccharide and bovine serum albumin (BSA, $\sim 66 \mathrm{kDa}$ ) (CAS. No. 9048-46-8) as protein were used as three different 
organic models to study the rejection and fouling behaviour of membranes. Glutaraldehyde $50 \% \mathrm{v} / \mathrm{v}$ for fixation the bacteria, and all related chemicals and biological agents used for bacterial tests (e.g. Phosphate Buffer Solution (PBS), nutrient agar medium, Mueller Hinton Broth (MHB), and neutraliser) were all purchased from Sigma-Aldrich (UK). Sodium chloride $(\mathrm{NaCl})$, sodium sulfate $\left(\mathrm{Na}_{2} \mathrm{SO}_{4}\right)$ and magnesium sulfate $\left(\mathrm{MgSO}_{4}\right)$ were analytical grades salts supplied by Fisher Scientific (UK). Cobalt (II) chloride $\left(\mathrm{CoCl}_{2}\right)$, manganese nitrate $\mathrm{Mn}\left(\mathrm{NO}_{3}\right)_{2} \cdot 4 \mathrm{H}_{2} \mathrm{O}$, nickel II acetate tetrahydrate $\left(\mathrm{Ni}\left(\mathrm{CH}_{3} \mathrm{COO}\right)_{2} \cdot 4 \mathrm{H}_{2} \mathrm{O}\right.$.), lead nitrate $\left(\mathrm{Pb}\left(\mathrm{NO}_{3}\right)_{2}\right)$, and copper nitrate $\left(\mathrm{Cu}\left(\mathrm{NO}_{3}\right)_{2} .3 \mathrm{H}_{2} \mathrm{O}\right)$ were supplied by Fisher Scientific (UK). $200 \mathrm{~nm}$ carboxylated polystyrene latex as tracer particles for surface zeta potential by LDE technique were supplied by Polysciences Inc., PA, USA. Two bacterial species; Escherichia coli (ACIB 8277) and Staphylococcus aureus (ATCC 6538P) were provided from Microbiology laboratories-Swansea University.

\subsection{Analytical and measuring instruments}

\subsubsection{Total organic carbon analyser (TOC)}

Total organic carbon analyser (TOC-L, Shimadzu, Japan) was employed to determine the total organic carbon content in the feed and permeate samples (Figure 3.1). Three organic fouling models (HA, NaAlg and BSA) and polyethylene glycol/oxide used during solute transport experiments were analysed by the TOC instrument.

The TOC system was calibrated once a year and regularly checked to ensure reliable measurements. A calibration curve was created using a standard solution of potassium phthalate solution - to represent the total carbon content (TC) and a mixture of sodium hydrogen carbonate and sodium carbonate - to represent the inorganic carbon (IC). A stock solution (1000 mg/l - concentration of carbon) was prepared for each TC and IC solution. Then, diluted to make a series of standard solution $(0,5,25,50,100$, 150, 200 and 250) $\mathrm{mg} / \mathrm{l}$ which cover the entire concentration range of tested solution (maximum concentration used in this paper was $200 \mathrm{mg} / \mathrm{l}$ ). These standards were measured and used to create the calibration curve. The unknown sample concentrations were linked to that curve via setting up method, and the TOC content is measured by subtraction of IC from TC in the samples. 


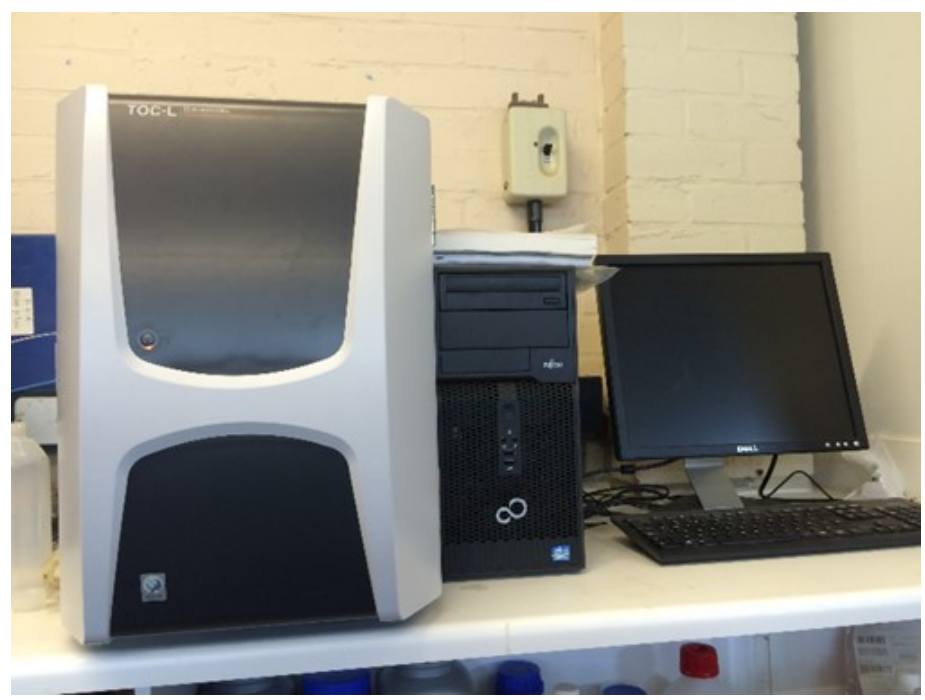

Figure 3.1: Total organic carbon analyzer (TOC-L, Shimadzu).

\subsubsection{Atomic absorption spectrometry (AAS)}

The heavy metals contents in the feed and permeate were determined by AAS instrument (PinnAAcle 900F). Prior to measurements, a method file was set up, and a series of diluted standard solutions, prepared earlier for each metal salt solution, was injected into the system to obtain the calibration curves, see Figure 3.2.

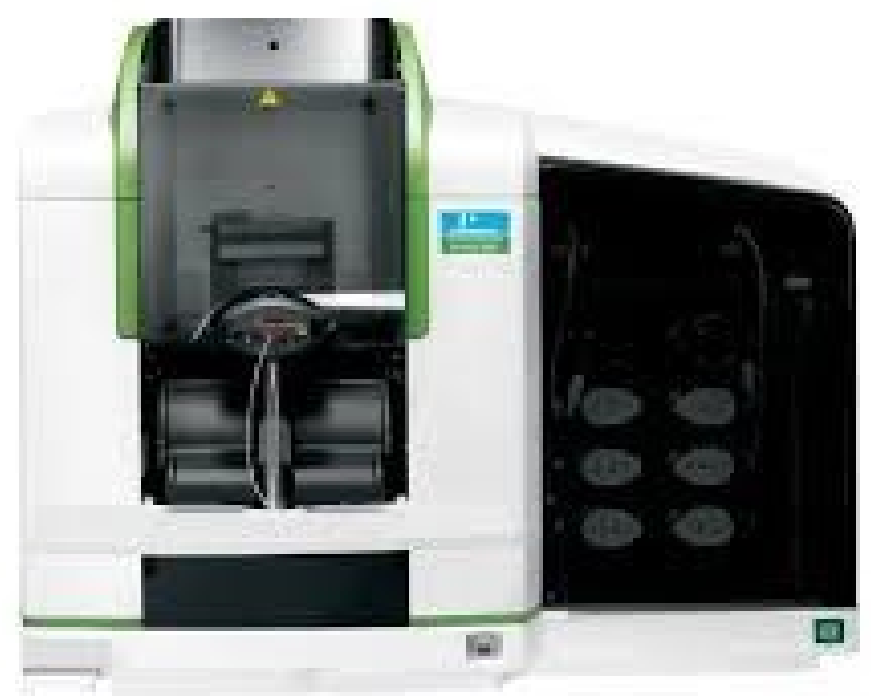

Figure 3.2: Atomic absorption spectrometer (PinnAAcle 900F). 


\subsubsection{Mechanical tester}

The mechanical properties, including the stress/strain relationship and elastic modulus of the prepared membranes, were determined using a tensile tester instrument (Instron 1162, UK), see Figure 3.3. The measurements were carried out according to ASTM D882-12 (as a closest standardised test) at ambient conditions $\left(25^{\circ} \mathrm{C}\right)$. Dry membranes samples were cut into a standard strap $(1 \mathrm{~cm} \mathrm{x} 4 \mathrm{~cm})$, and the measurements performed at a crosshead speed of $0.5 \mathrm{~mm} / \mathrm{min}$. For measurement reliability, triplicate measurements conducted and results presented as an average value. The Young's modulus (E) was calculated from the initial slop of stress/strain curve; for testing thin plastic sheets (thickness less than $1 \mathrm{~mm}$ );

$$
\mathrm{E}=\frac{d \sigma}{d \varepsilon} \text { at } \varepsilon=0
$$

Where the stress $(\sigma)$ is the force per cross-section area $\left(\mathrm{N} / \mathrm{m}^{2}\right)$ and $\varepsilon$ is the strain and calculated from the ratio of the change/deformation in the length $(\Delta L)$ to the initial length $\left(L_{\mathrm{o}}\right)$, as follow;

$$
\varepsilon=\frac{\Delta L}{L_{\mathrm{o}}}
$$

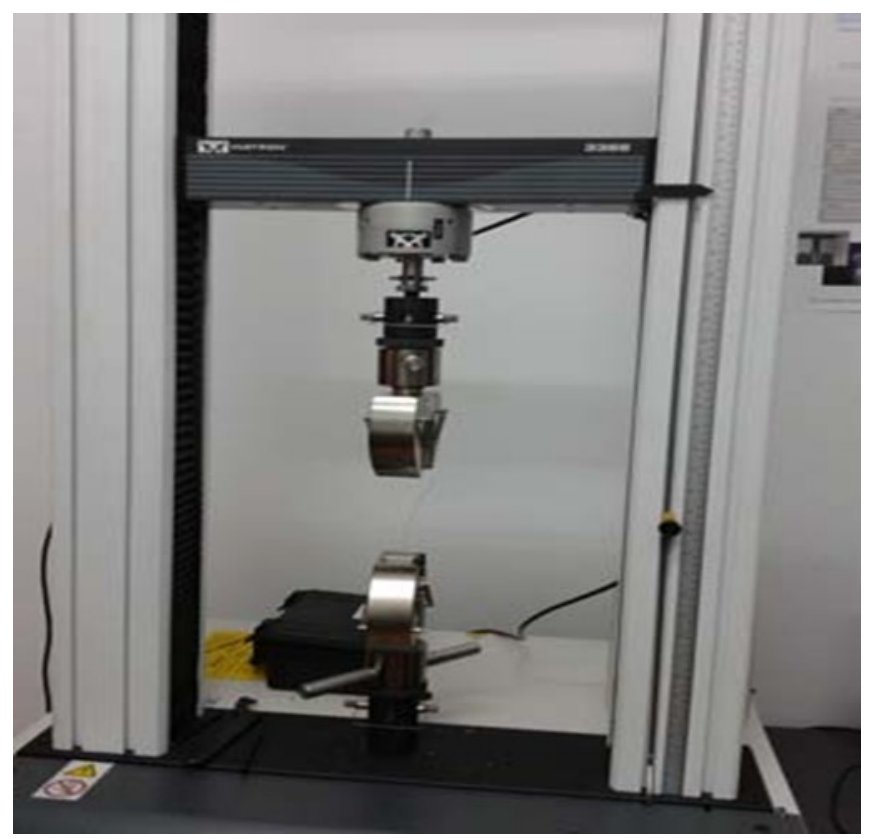

Figure 3.3: Instron - universal tensile tester instrument 


\subsubsection{Surface charge measurements}

\subsubsection{Membranes surface charge techniques}

Two techniques, based on different theories, were employed to evaluate the charge along the surface of the membranes, as follows;

\section{Tangential streaming potential (TSP)}

Tangential streaming potential measurements were carried out using an Electrokinetic Analyzer (EKA) (Anton- Paar GmbH, Austria), as shown Figure 3.4. The streaming potentials measurements were performed in flat-sheet flow module. Two flat-sheet membranes to be investigated were cut to fit the dimensions $(5.5 \times 12.5 \mathrm{~cm})$ and attached to the rectangular measurement module. The membrane samples were placed with their active layers facing each other and separated by a PTFE gasket with a flow channel inside.

Before measurements, the EKA system was rinsed with DI water, then flushed by fresh electrolyte $(10 \mathrm{mM} \mathrm{NaCl})$ solution to equilibrate the membrane sample. The background electrolyte solution conductivity and $\mathrm{pH}$ were monitored, and measurements were conducted based on Helmholtz-Smoluchowsky Equation (3.1)

$$
\zeta=\frac{\Delta E \mu k}{\Delta P \varepsilon_{0} \varepsilon_{r}},
$$

where $\Delta E$ is the streaming potential, $\mu$ is the viscosity of the solution, $k$ is conductivity of electrolyte solution, $\Delta P$ is the transmittance pressure drop, $\mathcal{E}_{0}$ is the permittivity of a vacuum, and $\mathcal{E}_{r}$ is the dielectric constant of the medium at $25^{\circ} \mathrm{C}$.

The measurements performed with $\mathrm{pH}$ range (3-11). The $\mathrm{pH}$ has periodically adjusted for alkaline and acidic $\mathrm{pH}$ using $0.1 \mathrm{M} \mathrm{HCl}$ and $0.1 \mathrm{M} \mathrm{NaOH}$. Streaming current was generated by applying a pressure gradient program of 0-700 mbar over 30 seconds and measured using a pair of $\mathrm{AgCl}$ electrodes. At each $\mathrm{pH}$ point, 10 measurements were conducted at room temperature. 


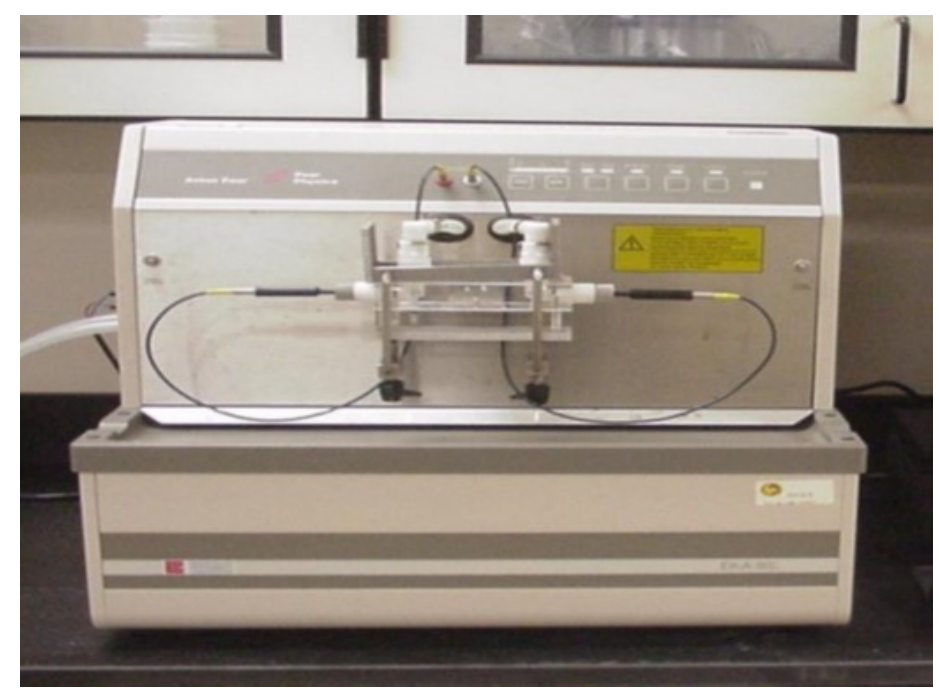

Figure 3.4: Electrokinetic Analyzer (EKA) (Anton- Paar GmbH, Austria)

\section{Laser Doppler Electrophoresis (LDE)}

Electrophoretic mobility and electro-osmotic measurements were performed using a surface zeta potential cell (ZEN1020), as an accessory kit for the Malvern Zetasizer ZS nanoseries instrument (Malvern Instruments Ltd, Malvern, UK), see Figure 3.5 and Figure 3.6. Dry membrane's sample was cut into 5.0 x $3.5 \mathrm{~mm}$ (rectangular dimension) and attached to a sample holder using an appropriate adhesive (a water-resistant epoxy or double sticky tape), and the sample holder was then loaded to the surface cell. Once the sample holder is in the place, a height alignment tool is used to align the simple's surface to a zero height with respect to the instrument laser. After this coarse alignment performed, the surface cell inserted into a glass cuvette filled with $1 \mathrm{ml}$ electrolyte solution $(10 \mathrm{mM} \mathrm{NaCl})$ contains $0.2 \mu \mathrm{m}$ carboxylated polystyrene latex (tracing particles). Before placing the cell in the instrument, the covette was inspected to assure no air bubbles has presented on the cell's electrode. At this stage, a final fine adjustment of zero position is carried out using the count rate meter in the zetasizer application software. Finally, SOP file was configured and measurement was performed at 4 different displacements $(125,250,375$ and $500 \mu \mathrm{m})$ from the membrane surface. At each measurement point, 4 runs were performed (each run consists 15 sub-runs). Following these measurements, a final measurement was used to determine the electroosmotic mobility of latex particles at a far distance from the membrane's sample. At the end of the measurements, an intercept could be extrapolated by plotting electrophoretic mobility or zeta potential of the tracers at multiple distances away from 
the membrane surface, and the surface zeta potential of the membrane was determined according to Equation 3.4 below;

$$
\zeta(\text { surface })=- \text { intercept }+\zeta(\text { tracing particle }), \quad 3.4
$$

In between subsequent measurements, the cell electrodes were sonicated in toluene and rubbed with cotton swaps to eliminate any possible debris accumulated on electrodes during the previous measurement.

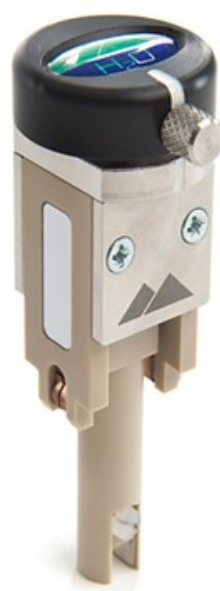

Figure 3.5: Surface zeta potential cell (ZEN1020).

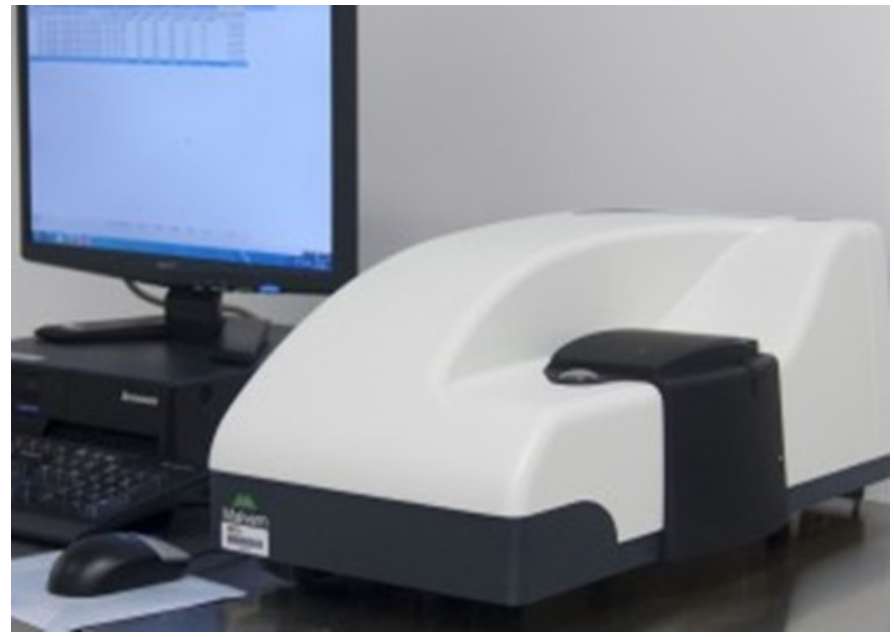

Figure 3.6: Malvern Zetasizer ZN nanoseries instrument (Malvern Instruments Ltd, Malvern, UK) 


\subsubsection{Nanomaterials surface charge}

The zeta potential $(\zeta)$ measurements were performed using a universal dip cell kit (ZEN1002), as an accessory for the Malvern Zetasizer ZN nanoseries instrument (Malvern Instruments Ltd, Malvern, UK), shown in Figure 3.7. Prior to measurement, the dip cell electrodes were immersed in a gentle ultrasound bath for $5 \mathrm{~min}$ to remove any contaminations from previous measurements. A concentration of $5 \mathrm{mg} / \mathrm{l}$ for each nanomaterial sample was dispersed in water by tip sonicator for $40 \mathrm{~min}$, and the $\mathrm{pH}$ was titrated from 3 to 11 using $0.1 \mathrm{M} \mathrm{HCl}$ and $0.1 \mathrm{M} \mathrm{NaOH} .1 \mathrm{ml}$ of the solution was then poured into a disposable cuvette and the cell was carefully inserted to avoid any chance of bubble formation between the palladium electrodes of the cell. During the measurement, the incident laser beam passes through the sample, and the scattered light is detected at a forward angle of $13^{\circ}$. When applying the electrical field to the dip cell, a fluctuation of the light is caused by moving particles. The zeta potential was calculated by determining the electrophoretic mobility of particles and then applying Henry equation (Equation 3.5) by considering the Smoluchowski approximation. The electrophoretic mobility can be obtained by carrying an electrophoresis experiment on the sample and measuring the velocity of the particles by Laser Doppler velocimetry.

$$
\mathrm{U}_{\mathrm{E}}=\frac{2 \varepsilon \zeta \mathrm{f}(\mathrm{ka})}{3 \eta}
$$

Where;

$\mathrm{U}_{\mathrm{E}:}$ Electrophoretic mobility.

$\mathcal{E}$ : Dielectric constant.

$\zeta$ : zeta potential.

$\eta$ : Viscosity.

$f(k a$; Henry's function. 


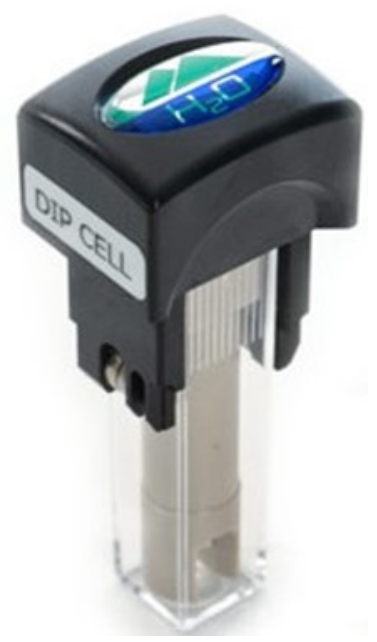

Figure 3.7: Universal dip cell kit (ZEN1002).

\subsubsection{Contact angle (CA)}

Surface hydrophilicity/hydrophobicity of the membranes was evaluated via a sessile drop method using VCAoptima contact angle instrument (AST Products, MA - USA), see Figure 3.8. Membrane samples were cut into $1 \times 6 \mathrm{~cm}$ straps and attached to a glass microscope slides via double sticky tape to flatten the membrane samples. $4 \mu 1$ droplets of DI water are dropped onto the flat membrane's surface, and an image of the droplet was automatically captured to determine the left and right contact angle using VCA Optima XE software. At least ten measurements for three membranes replicates were recorded, and the results were averaged.

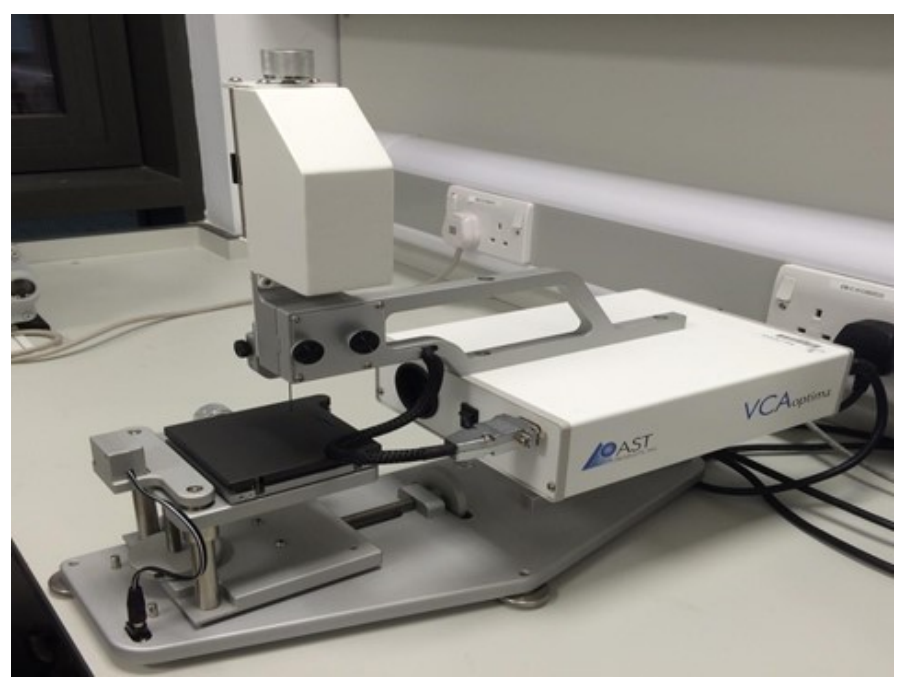

Figure 3.8: VCAoptima contact angle instrument, (AST Products, MA - USA). 


\subsubsection{Atomic Force Microscopy (AFM)}

Atomic force microscopy (AFM) imaging was used to characterise the surface topography parameters of membranes, using a multimode AFM with a NanoScope IIIa controller (Bruker, USA), see Figure 3.9. The analysis was conducted by tapping mode in the air at $25{ }^{\circ} \mathrm{C}$ using 0.01-0.025 Ohm-cm Antimony (n) doped Silicon cantilever PESP-V2 (Bruker, USA).

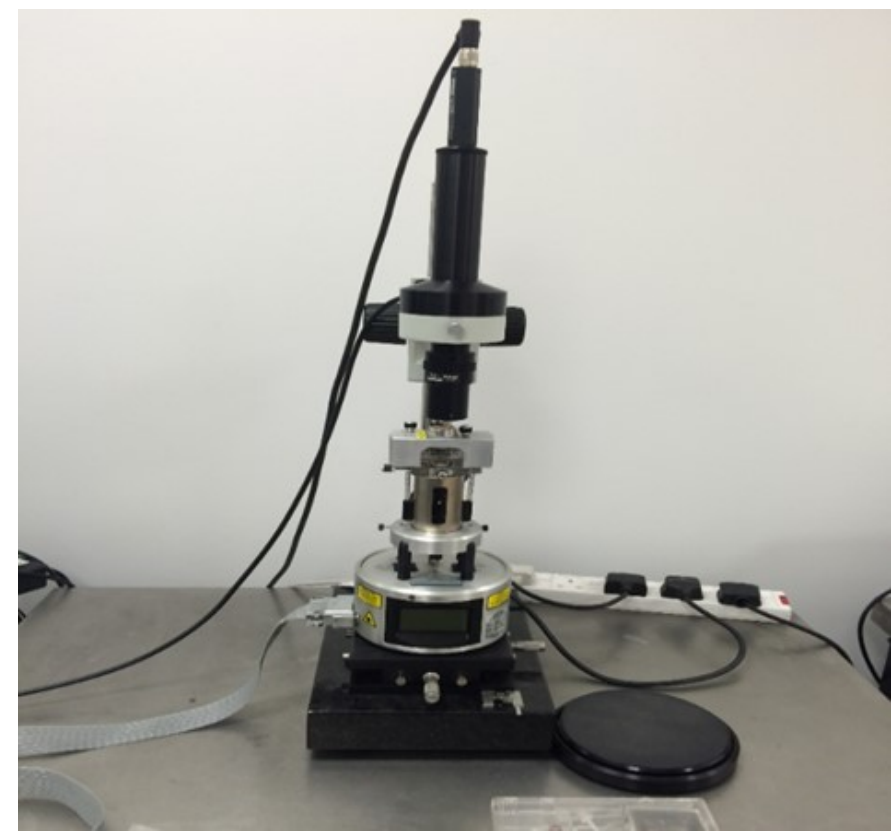

Figure 3.9: Atomic force microscopy (AFM), (Bruker, USA).

The basic working principle of the AFM is to let a sharp microscopic cantilever's tip to scan the surface topography of a chosen area of interest. As this sharp tip scans across the sample surface, various topography features cause a deflection of that tip. Subsequently, a light beam generated by a laser diode is bounced off of the back of the cantilever and reflected to a four section position-sensitive photodiode to detect any sub-angstrom vertical movement caused by the tip. Indeed, generated a map for the surface topography through software supplied by the manufacturer and used for this purpose, as illustrated in Figure 3.10. 


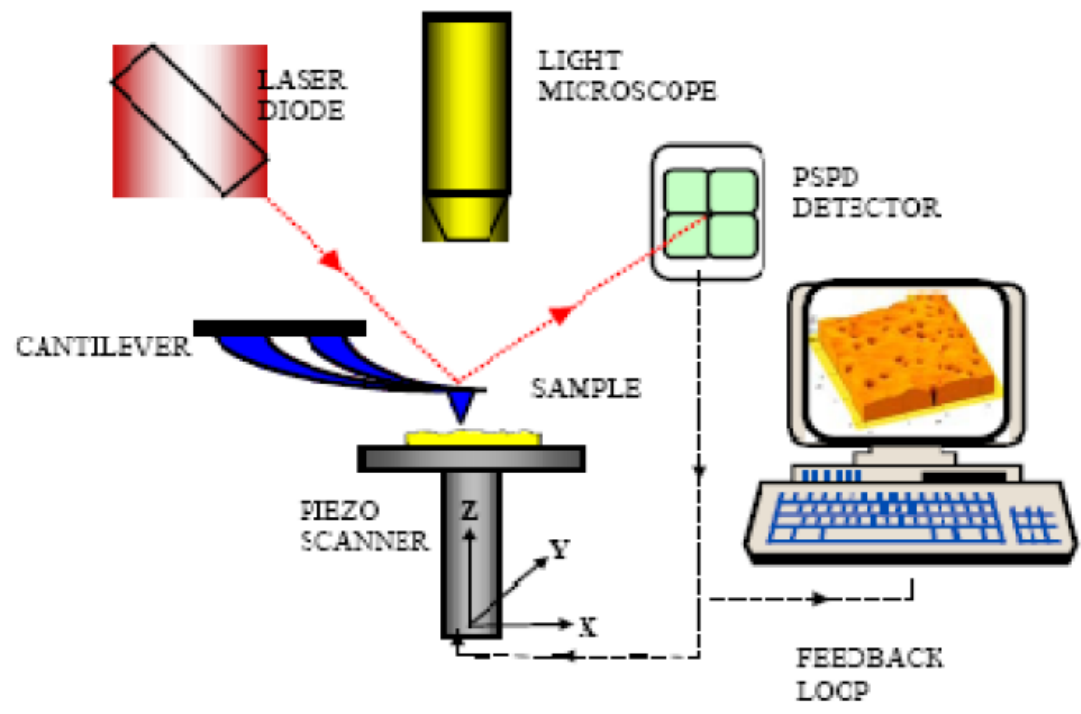

Figure 3.10: Schematic demonstration of the principle of AFM operation.

The imaging procedure starts by fixation of a dry and clean membrane sample on a small steel disc using adhesive tape from both sides. Thereafter, the disc held on the AFM sample holder for imaging. The surface topography parameters obtained from membranes imaging by AFM can be summarised as follows:

i. The root mean square roughness (RMS) defined as the square root of the mean value of the squares of the distance of the points from the image mean value:

$$
\mathrm{RMS}=\sqrt{\frac{1}{\mathrm{~N}}} \sum_{\mathrm{i}=1}^{\mathrm{N}}\left\langle\mathrm{Z}_{\mathrm{i}}-\overline{\mathrm{Z}}\right\rangle^{2},
$$

ii. The average roughness $(\mathrm{Ra})$ which defined as the arithmetic mean of the deviation in height from the image to the mean value as shown:

$$
\mathrm{Ra}=\frac{1}{\mathrm{~N}} \sum_{\mathrm{i}=1}^{\mathrm{N}}\left|\mathrm{Z}_{\mathrm{i}}-\overline{\mathrm{Z}}\right|
$$

iii. The average mean height $(\mathrm{AV}$ mean $)$ is an arithmetic mean defined as the sum of all height values divided by the number of data points:

$$
|\mathrm{Z}|=\frac{1}{\mathrm{~N}} \sum_{\mathrm{i}=1}^{\mathrm{N}} \mathrm{Z}_{\mathrm{i}},
$$


Where; $Z_{\mathrm{i}}$ : current $\mathrm{Z}$ value, $\mathrm{Z}$ : an average of $\mathrm{Z}$ value, $\mathrm{N}$ : number of points within the area.

\subsubsection{Scanning Electron Microscope (SEM) and (EDX)}

Scanning electron microscopy (SEM S4800- Hitachi, Japan) was employed to observe the surface and/or cross-section morphology of samples as shown in Figure 3.11A. Whilst energy dispersive X-ray analysis (EDX) attached to the SEM was implemented to provide a quantitative representation for the elemental composition of various samples.

Prior to cross-section observation, membranes samples were immersed in liquid nitrogen for a couple of minutes and fractured while maintained in liquid nitrogen temperature. The fractured samples were then coated with a thin film of $5 \mathrm{~nm}$ chromium (Cr) carried by a sputter coater machine (Quorum, Q150T ES, UK), see Figure 3.11B. Finally, the sample was mounted on a stainless-steel sample holder (designed for crosssection imaging), placed inside the SEM chamber and imaged at variant accelerating voltages and under high vacuum. For membrane surface imaging, only coating with $\mathrm{Cr}$ was carried to make it conductive, and another coating layer could be applied if necessary. For NMs, about $1 \mathrm{mg}$ of the powder was stuck firmly on double sticky tape, mounted on the stub and introduced for imaging.
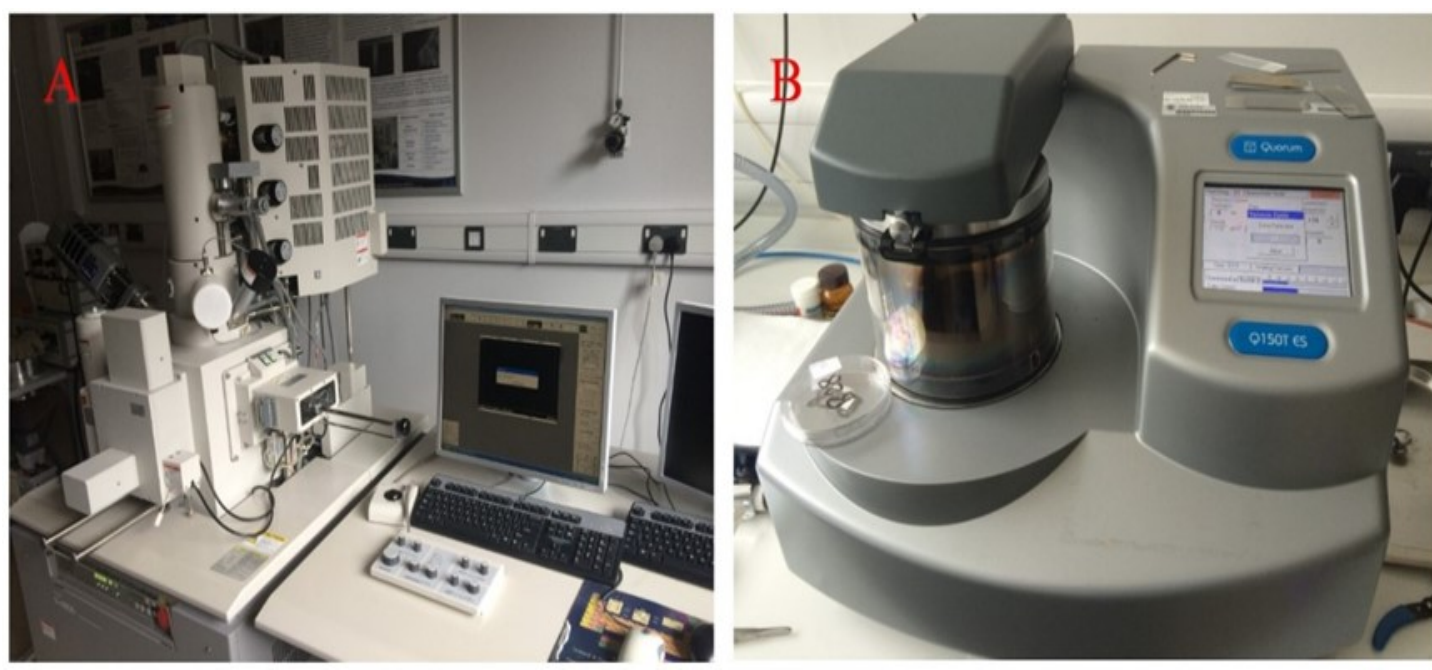

Figure 3.11: (A) Scanning electron microscopy (SEM S4800- Hitachi), and (B) sputter coater machine (Quorum, Q150T ES). 


\subsubsection{Thermogravimetric analysis (TGA)}

Thermal gravimetric analysis (TGA) was performed using TA instrument (Q600SDT, USA), as shown in Figure 3.12. The Q600 features a highly reliable horizontal dualbalance mechanism that supports precise TGA measurements. It delivers superiority in weight signal measurements (accuracy, sensitivity, and precision) over what is available from single beam devices since the dual beam design virtually eliminates beam growth and buoyancy contributions to the underlying signal. The machine delivers a measurement of the heat flow and weight changes associated with transitions and reactions in materials over the temperature range ambient to $1500^{\circ} \mathrm{C}$. The balance was tared before loading the sample, and an alumina pan $(40 \mu \mathrm{L})$ was filled with approximately $5 \mathrm{mg}$ of powder sample and laid on a thermocouple beam to load it into the combustion chamber. The sample was then heated in the presence of air up to 900 ${ }^{\circ} \mathrm{C}$ at heating rate $10{ }^{\circ} \mathrm{C} / \mathrm{min}$ and air flow rate $100 \mathrm{ml} / \mathrm{min}$. In the meantime, the correlation between temperature and weight percent loss has been plotted by the TGA software.

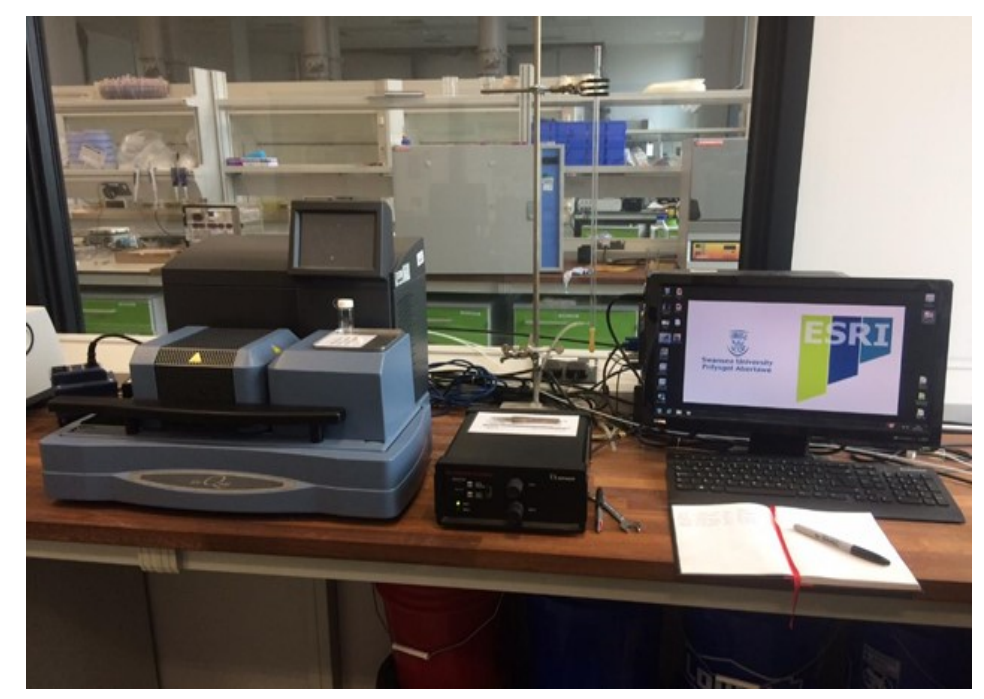

Figure 3.12: Thermal gravimetric analysis (TGA) - TA instrument (Q600SDT).

\subsubsection{Fourier transform infrared spectrophotometry (FT-IR)}

To affirm the existence of functional groups on the surface of MWCNT, and molecular structures of TFN membranes surface, a Spectrum Two FT-IR spectrometer (PerkinsElmer, USA) was implemented, see Figure 3.13 below. 


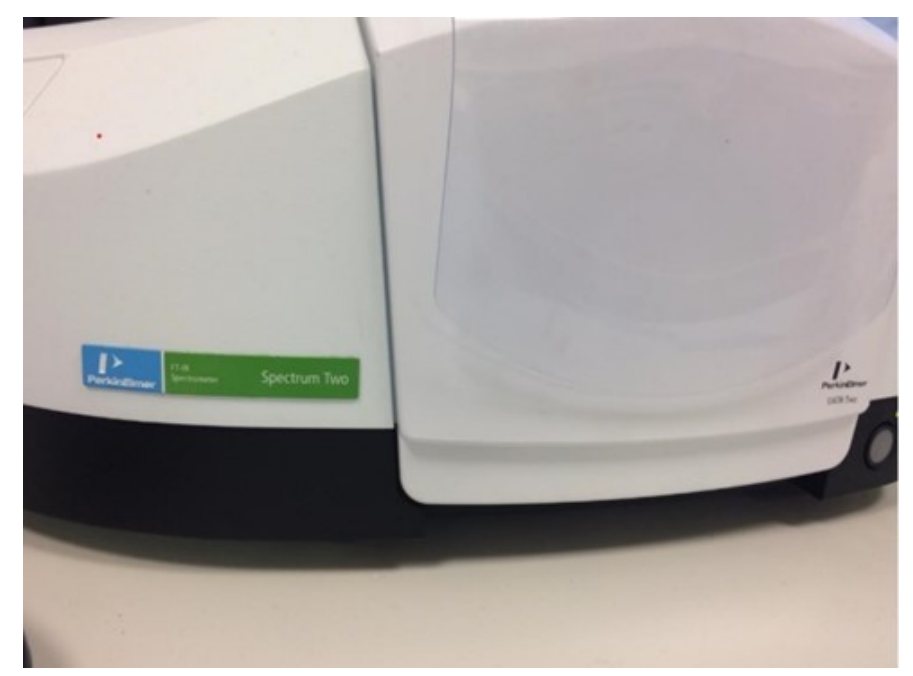

Figure 3.13: FTIR - Spectrum Two - (PerkinsElmer, USA).

The working principle of FT-IR is illustrated schematically in Figure 3.14. An IR beam is emitted from an irradiation source. Then, the beam passes through into interferometer which performs the spectral encoding. A destructive and constructive interference called an interferogram has been created from the recombination of different path length-beams in the interferometer. Now, as the beam passes through the sample, specific frequencies of energy are absorbed by the sample, which are characteristics of the sample from interferogram. At this stage, the detector measures the interferogram signal (in energy vs time) for all frequencies. In the meanwhile, a bean is superimposed to create a reference (background) spectrum. And the final spectrum was obtained after the interferogram had subtracted the background spectrum from the sample spectrum by the computer software. Before using the spectrometer, the crystal was wiped with isopropanol to avoid any possible contamination and left to dry. Then, a background spectrum has been recorded while the arm is not over the crystal. Subsequently, a small amount of the sample is placed on the plate (just to cover the crystal), and the final spectrum of the sample is recorded. The transmittance spectra were recorded at room temperature from 400 to $4000 \mathrm{~cm}^{-1}$ with a resolution of $4 \mathrm{~cm}^{-1}$. 


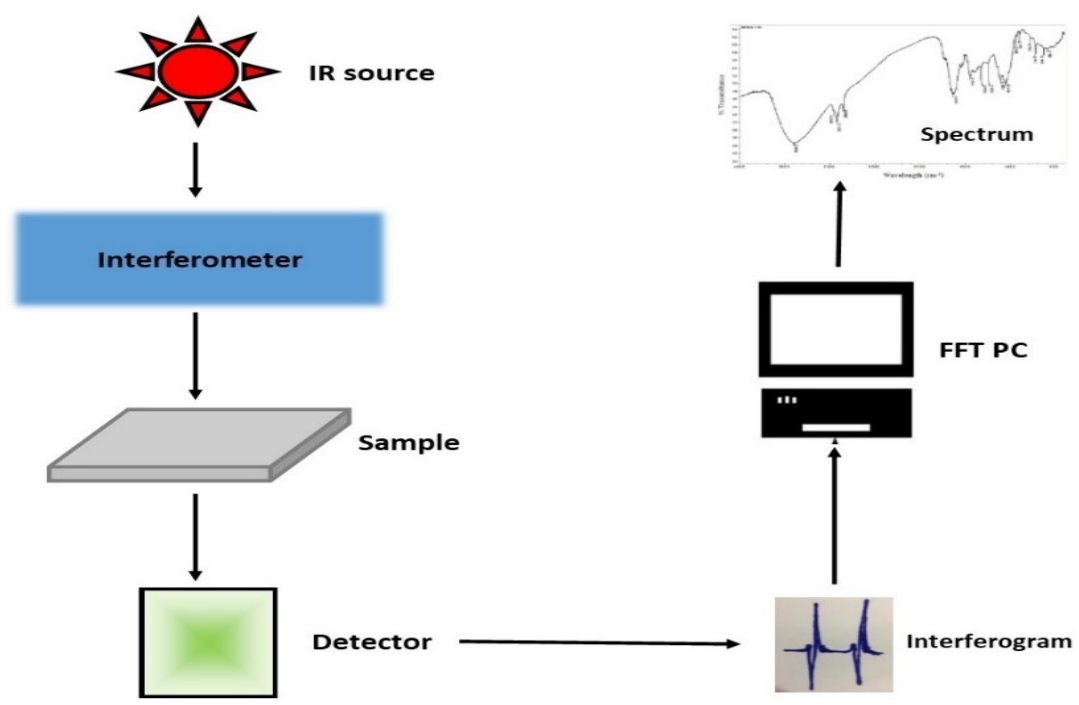

Figure 3.14: The basic component in FT-IR spectrometer

\subsubsection{Raman spectroscopy}

Raman spectroscopy, as a non-destructive technique, was applied to evaluate the graphitic structure, defects, ordered and disordered structures of HNS. The Raman spectra were recorded for samples on $\operatorname{InVia}^{\mathrm{TM}}$ Confocal Raman Microscope (Renishaw-UK) with a $532 \mathrm{~nm}$ excitation laser (Figure 3.15). Measurements were conducted for $10 \mathrm{~s}$ exposure, $10 \%$ intensity, and $50-3500 \mathrm{~cm}^{-1}$ spectrum range.

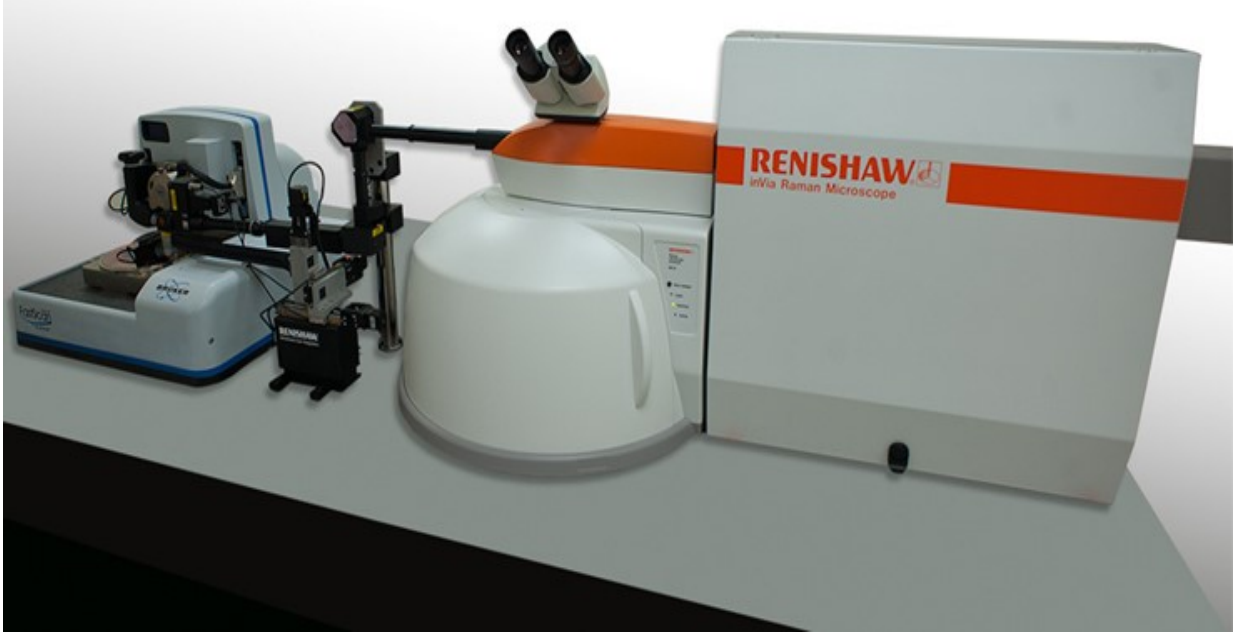

Figure 3.15: InVia ${ }^{\mathrm{TM}}$ Confocal Raman Microscope (Renishaw). 


\subsubsection{X-ray diffraction (XRD)}

The crystalline structures of the M/MO nanoparticles and the nanotubes prior and after the functionalization were determined by X-ray Diffraction (XRD), see Figure 3.16 below. XRD Patterns were recorded on a Brüker d8 DISCOVER diffractometer with a $\mathrm{Cu}$ ka X-ray source $(\mathrm{k}=0.15418 \mathrm{~nm})$ and analysed using Match 2 software.

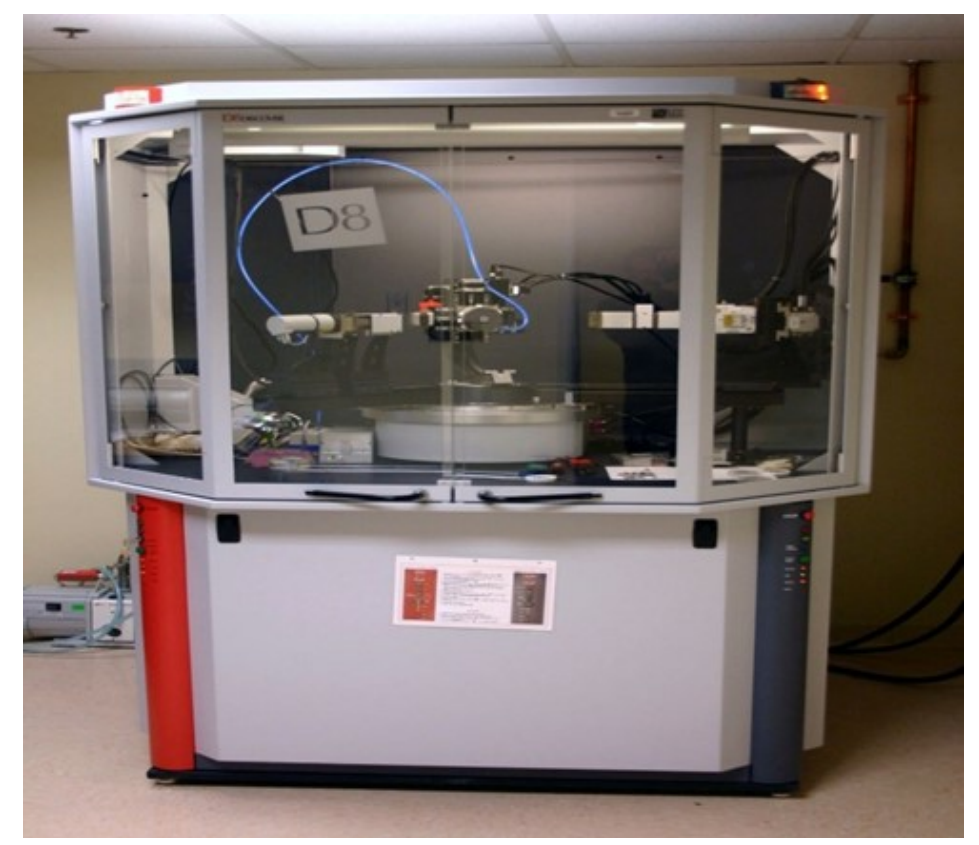

Figure 3.16: Brüker d8 DISCOVER diffractometer.

\subsubsection{X-ray photoelectron spectroscopy (XPS)}

Elemental composition and chemical state were further characterised by XPS analysis. An X-ray photoelectron spectrometer (Kratos, AXIS SUPRA - Japan), see Figure 3.17. The XPS equipped with a hemispherical analyser and monochromatic Al Ka X-ray $(1486.6 \mathrm{eV})$ as the radiation source, was used to identify the nanoscale materials from a range of $0-1200 \mathrm{eV}$. CasaXPS software was used for data analysis. A calibration of spectrum energy was carried out with respect to $\mathrm{C} 1$ peak, and binding energy (BE) set to $284.8 \mathrm{eV}$. 


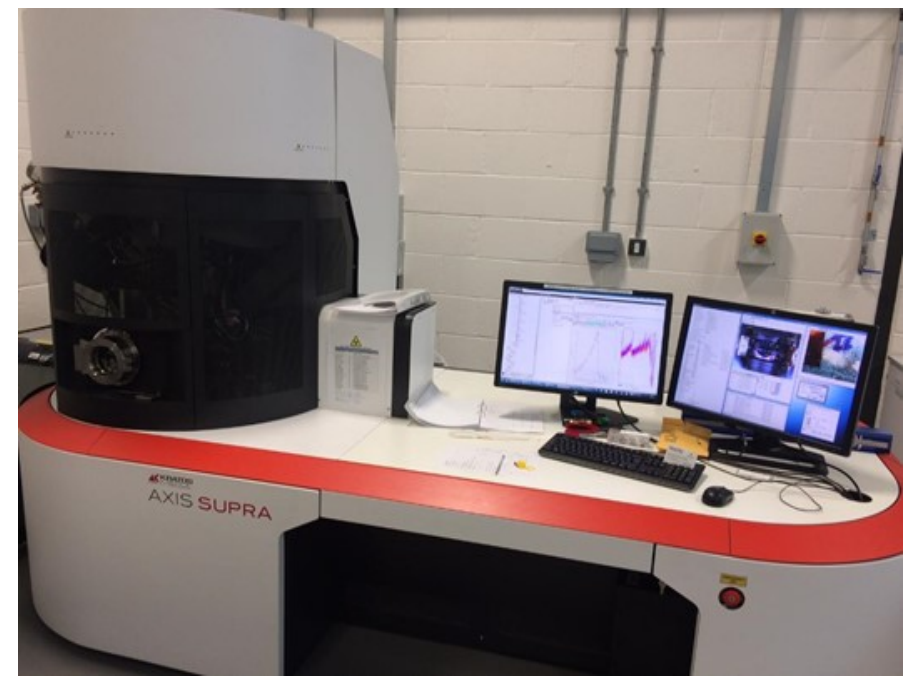

Figure 3.17: X-ray photoelectron spectrometer (Kratos, AXIS SUPRA).

\subsubsection{Probe sonicator}

A powerful probe sonicator (Vibra cell TM - Sonics, USA) with $120 \mathrm{~W}$, has been employed to achieve a high degree of dispersion for various HNS (Figure 3.18). all measurements were carried at $80 \%$ amplitude and 2 seconds pulse.

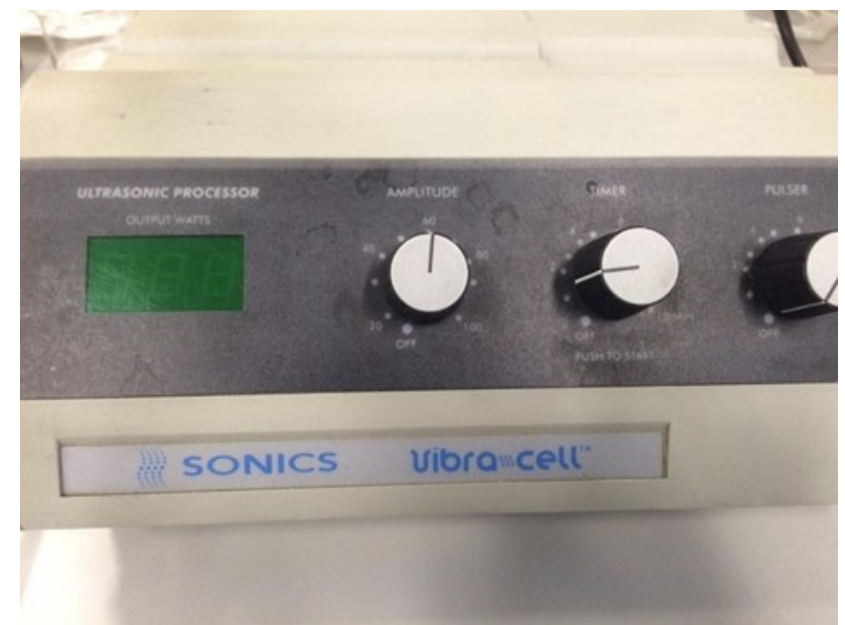

Figure 3.18: Tip sonicator (Vibra cellTM - Sonics, USA)

\subsubsection{Microplate reader}

The concentration of Bactria dilutions was determined by measuring their turbidity at 600 OD using (Synergy H1 hybrid, BMW Labtech, Germany), see Figure 3.19 below. 


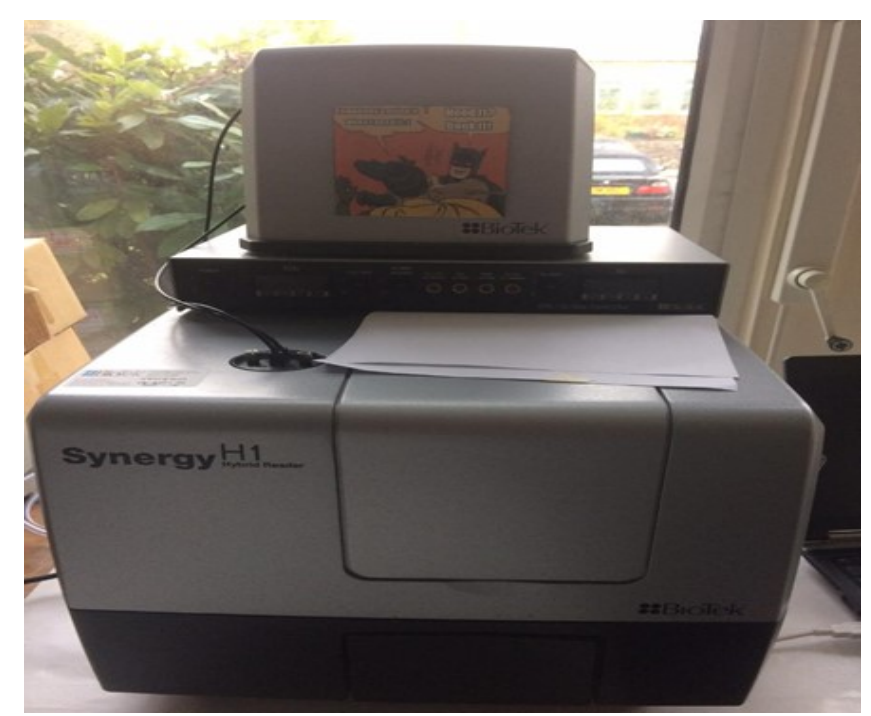

Figure 3.19: Microplate reader (Synergy H1 hybrid, BMW Labtech).

\subsubsection{Small tube furnace}

The HNS samples were calcinated inside a small high-temperature tube furnace (Carbolite Gero, UK), shown in (Figure 3.20). The Calcination was carried by placing the sample in a ceramic pan in the middle of a glass tube. The tube was then placed in the furnace and connected from one side to an inert gas source $\left(\mathrm{N}_{2}\right.$ or Ar) and left open from the other side. The inert gas flow rate was adjusted by the gas flow meter to prevent blowing the sample. The measurements were conducted for $4 \mathrm{hr}$ calcination time, $450{ }^{\circ} \mathrm{C}$ and $10{ }^{\circ} \mathrm{C} / \mathrm{min}$ temperature ramp. Finally, when the measurement is finished, the furnace was left to cool down to room temperature and the sample is collected.

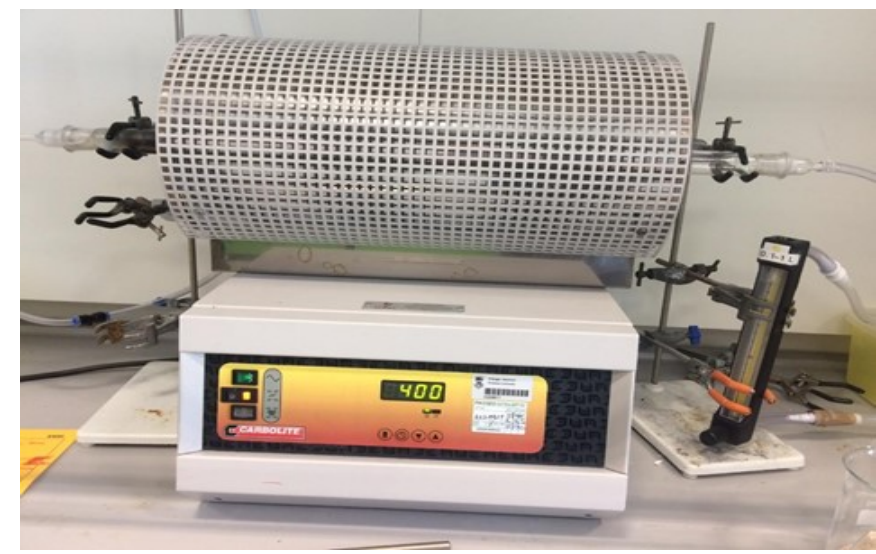

Figure 3.20: High-temperature tube furnace (Carbolite Gero, UK). 


\subsection{Experimental procedures}

\subsubsection{Membrane fabrication}

All UF membranes were fabricated via the classical non-induced phase separation (NIPS) technique. In brief, for membranes fabrication, a fixed amount (2 wt.\%) of PVP K40 was dissolved in NMP using a double neck-round bottom flask. The PES flakes were then gradually added to the solution and mechanically mixed overnight at $50{ }^{\circ} \mathrm{C}$ until a homogenous, yellowish and clear solution was achieved (Figure 3.21).

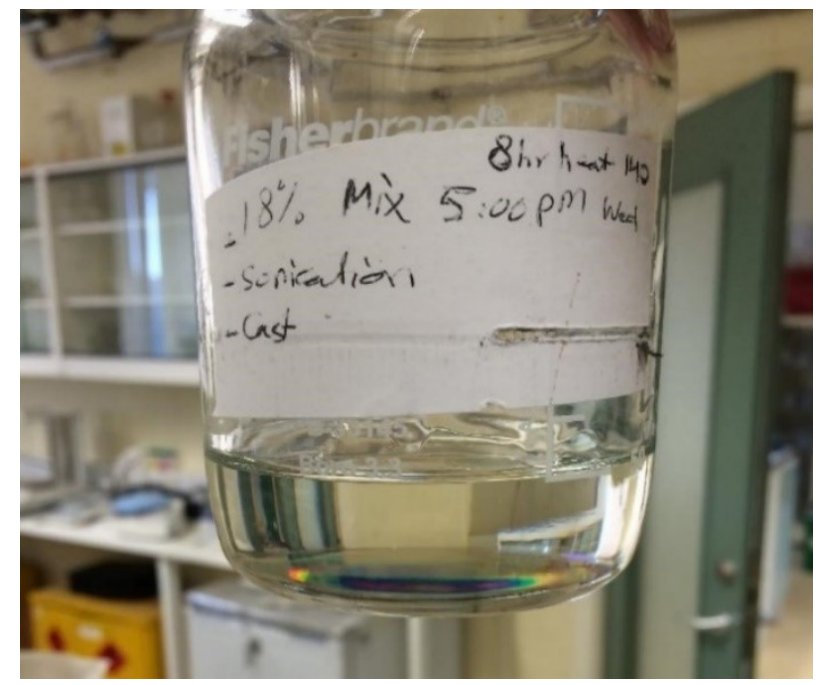

Figure 3.21: Dope casting solution (PES/NMP/PVP)

The casting solution was then degassed under vacuum for an hour to get rid of air bubbles. For solution casting, about $10 \mathrm{ml}$ of casting solution was poured onto a glass substrate and cast at a regular shear rate $\left(225 \mathrm{~s}^{-1}\right)$ via an automated casting knife (RK film applicator), as shown in Figure 3.22. The resultant thin film was directly placed in a DI water bath at $20^{\circ} \mathrm{C}$ for precipitation. Within less than two minutes, the membrane was detached from the glass substrate indicating that the phase inversion was completed. The membranes were then repeatedly rinsed with DI water to remove residual solvent and stored wet at $4{ }^{\circ} \mathrm{C}$ until used. All membranes were cast with 200 $\mu \mathrm{m}$ clearance gap at ambient temperature and relative humidity $(\mathrm{RH} \% 45 \pm 5)$. Prior to testing, the membranes were inspected under light to make sure there were no pinholes, wrinkles or any defect that could produce a performance discrepancy. This could be achieved whenever there is a well-mixed, air and dust free casting solution. Even though intense care has been taken during the fabrication, still there would be a 
variation in membranes performance between batch and another, and within the same membrane sheet at different locations. In this work, intensive efforts were devoted to maintain minimum uncertainty $(\leq 10 \%)$.

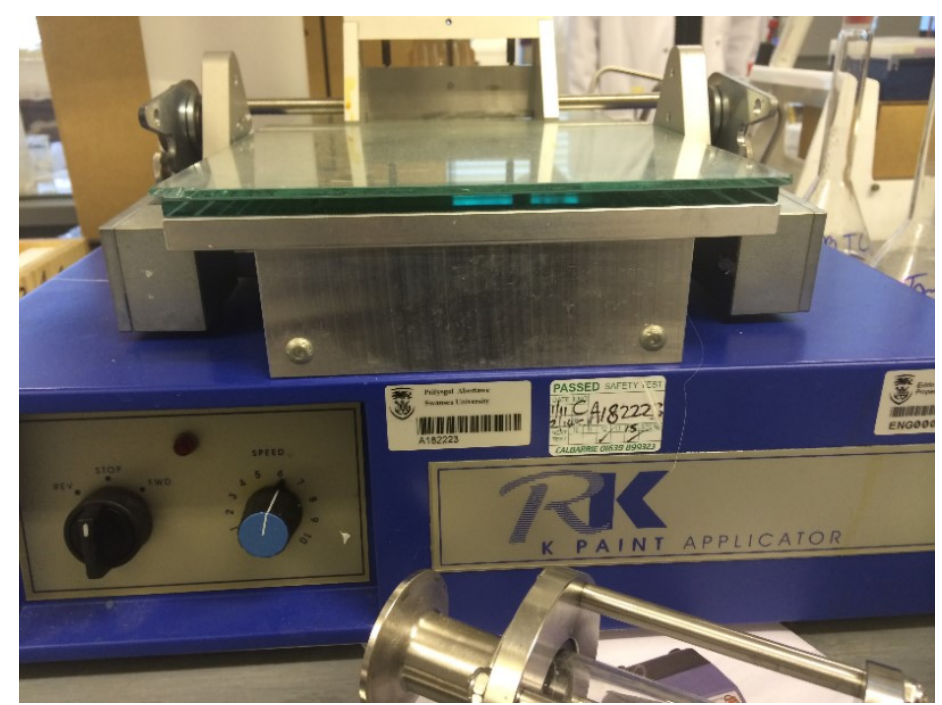

Figure 3.22: Automated casting knife (RK film applicator).

\subsubsection{Fabrication of hybrid nanostructures}

Prior to the decoration of MWCNTs with M/MO, the raw nanotubes were purified and functionalized by $\mathrm{HNO}_{3}$ acid treatment. More details are given in Chapter five, Section 5.2 .

\subsubsection{Stock solutions preparation}

\section{Organic foulants}

A 1000 ppm stock solution of three synthetic organic models (HA, SA and BSA) were prepared individually and used to characterise the performance of the fabricated membranes, as follow;

First, for the ease of humic acid dissolution, a $1 \mathrm{~L}$ measuring flask was filled with $1 \mathrm{~L}$ of DI water and adjusted to $\mathrm{pH} 9.5 \pm 0.1$, then quickly wrapped with aluminium foil. Thereafter, $1 \mathrm{~g}$ of humic acid powder was dissolved and stirred vigorously for at least 4 hours using magnetic stirrer. Finally, the solution was passed through $0.45 \mu \mathrm{m}$ membrane filter (Millipore, France) to remove undissolved fractions. While in the case of BSA, $1 \mathrm{~g}$ of the material was dissolved in $1 \mathrm{~L} \mathrm{DI}$ water without mixing, since mixing 
may form a foam and denature the BSA indeed. Therefore, the solution was left for 1 $\mathrm{hr}$ in a fridge to ensure a full dispersion. The polysaccharide, SA was mixed for $4 \mathrm{hr}$ under vigorous stirring speed and left overnight to achieve complete dispersion before use. All stock solutions are stored in a fridge at $4 \mathrm{C}^{\mathrm{o}}$ and consumed within 10 days.

\section{Heavy metals}

A $1000 \mathrm{ppm}$ as a standard solution of five different metal salts was prepared individually. 2.271, 4.595, 4.326, 1.614 and $2.537 \mathrm{~g}$ of $\mathrm{CoCl}_{2}, \mathrm{Zn}\left(\mathrm{NO}_{3}\right)_{2} .6 \mathrm{H}_{2} \mathrm{O}$, $\mathrm{Ni}\left(\mathrm{CH}_{3} \mathrm{COO}\right)_{2} \cdot 4 \mathrm{H}_{2} \mathrm{O}, \mathrm{Pb}\left(\mathrm{NO}_{3}\right)_{2}$ and $\mathrm{CuSO}_{4}$ respectively were dissolved in a hot (70 ${ }^{\circ} \mathrm{C}$ ) DI water at acidic environment (below $\mathrm{pH} 3$ ). Prior to experiments, $50 \mathrm{ppm}$ from each solution was mixed thoroughly to obtain a concentration of $250 \mathrm{ppm}$ of heavy metal ions solution mixture.

\subsubsection{Permeability, retention and fouling measurements}

Pure water flux (PWF) and filtration experiments have been conducted under a crossflow condition with an active membrane area of $12.6 \mathrm{~cm}^{2}$. Feed solution temperature was maintained at $20 \pm 0.5^{\circ} \mathrm{C}$ using a refrigerated chiller (Grant, LTD6G, England), see schematic Figure 3.23. After specific compaction time (time depends on membrane process) and pressure (5 bar for UF and 12 bar for NF membranes), the pressure was reduced to a lower value. The DI water permeate flux recorded automatically every $1 \mathrm{~min}$, using data collection software (Win-Wedge 3.0) interfaced with an electronic balance (Precisa, XB3200C, Switzerland). The automated software converted the permeate weight data received from the balance into a flux and recorded the values on an excel spreadsheet for previously set membrane area and time intervals. For the evaluation of membrane performance, the permeate flux $\left(1 / \mathrm{m}^{2} . \mathrm{h}\right)$ decline was recorded as described earlier for PWF after the compaction with DI water. The relative flux $(\mathrm{RF})$ in $(\%)$ was used to express the correlation of the permeate flux to the pure water flux of the respective virgin membrane. While rejection values $(\mathrm{R} \%)$ of the membranes were determined by the Equation 3.9:

$$
R(\%)=\frac{C_{f}}{C_{p}} \times 100
$$


Where $C_{p}$ and $C_{f}$ are the foulant concentration in the permeate and feed solutions, respectively. The concentration of feed and permeate samples were analysed using appropriate instrument depending on the foulant type. In addition, total fouling $\left(\mathrm{F}_{\mathrm{T}}\right)$, reversible fouling $\left(\mathrm{F}_{\text {rev }}\right)$ and irreversible fouling $\left(\mathrm{F}_{\text {ir }}\right)$ of membranes are determined as below (Equations 3.10 to 3.13), where $\mathrm{J}_{0}, \mathrm{~J}_{1}$, and $\mathrm{J}_{2}$ are initial water flux of virgin membrane, solute flux and water flux of fouled membrane after water flushing, respectively (Vatanpour et al., 2011). All measurements were replicated to take into account the membrane variability.

$$
\begin{array}{ll}
\mathrm{F}_{\mathrm{T}}=\mathrm{F}_{\text {rev }}+\mathrm{F}_{\mathrm{ir}}, & 3.10 \\
\mathrm{~F}_{\mathrm{T}}(\%)=\frac{\mathrm{J}_{2}}{\mathrm{~J}_{\mathrm{o}}} \times 100, & 3.11 \\
\mathrm{~F}_{\mathrm{rev}}(\%)=\frac{\mathrm{J}_{2}-\mathrm{J}_{1}}{\mathrm{~J}_{\mathrm{o}}} \times 100, & 3.12 \\
\mathrm{~F}_{\text {ir }}(\%)=\frac{\mathrm{J}_{\mathrm{o}}-\mathrm{J}_{2}}{\mathrm{~J}_{\mathrm{o}}} \times 100, & 3.13
\end{array}
$$

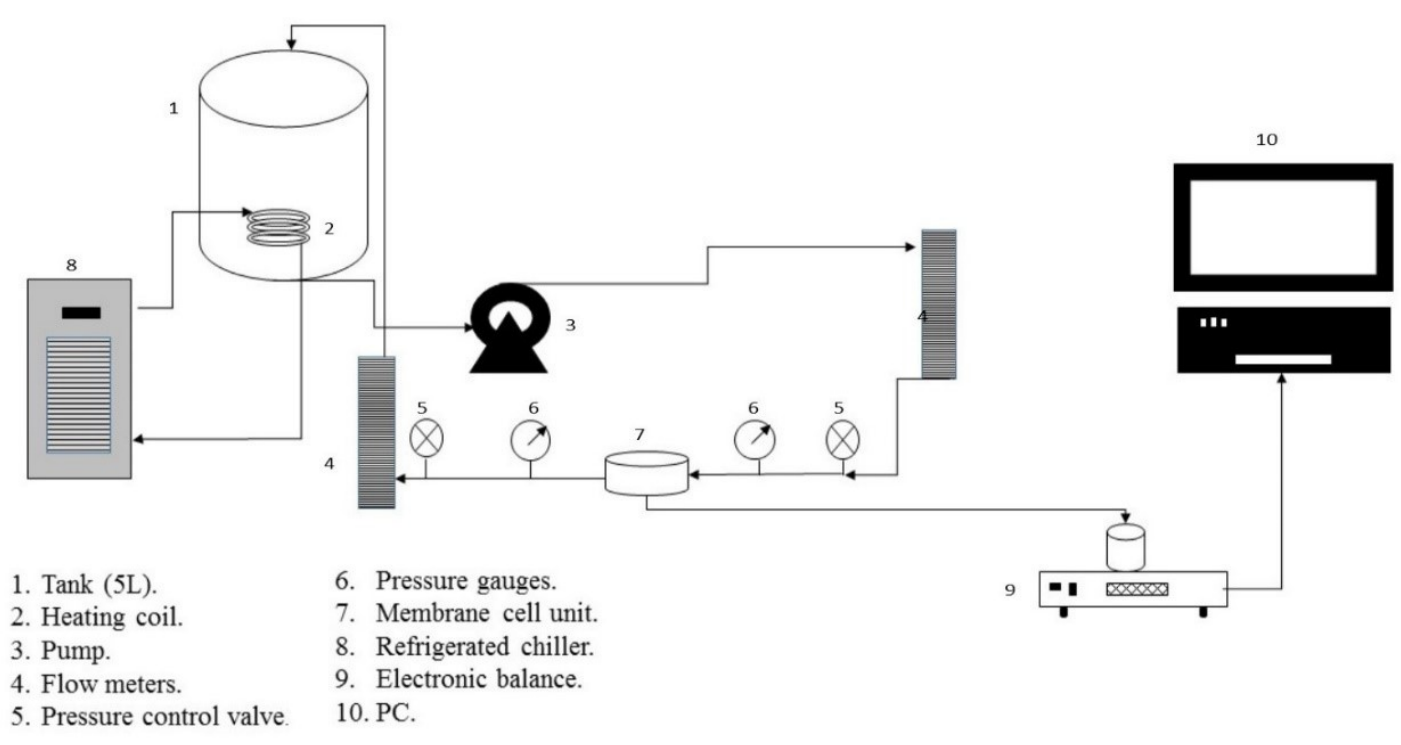

Figure 3.23: Schematic description of the cross-flow NF filtration unit 


\subsubsection{Solute transport method}

Mean pore size and pore size distribution (PSD) of membranes were determined using a solute transport mode as described by (Singh et al., 1998). This method is based on permeation-rejection correlation of a known solute size through membranes to determine the pore size of the pores open to flux, giving the minimal size of pore constrictions along the whole pore (Khayet \& Matsuura, 2003). Solute retention coefficients are based on the ratio of solute molecular size to membrane pore size (Paine \& Scherr, 1975). PEG/PEO as uncharged organic solutes with different molecular weights was used during the experiments. In all experiments, solution temperature and the transmembrane pressure were kept constant at $22 \mp 0.5^{\circ} \mathrm{C}$ and 4 bars, respectively. Following the compaction of membranes, a sequence of, $200 \mathrm{mg} / \mathrm{l}$ of five different molecular weight PEG solutions were passed through each membrane. For each membrane, the lowest PEG molecular weight aqueous solution was first introduced followed by the next higher one and so on until the highest molecular weight PEG. After each increase in PEG molecular weight, a sample from the permeate side was taken after $10 \mathrm{ml}$ of permeate had passed through the membrane. Both feed and permeate concentrations were analysed by a Total Organic Carbon analyser (Figure 3.1), whereas different PEG solute separation percents were calculated via Equation 3.9 .

The dimension of solutes is described by the stoke radius since the solute molecular size cannot be easily obtained using direct measurements. So, the Stokes-Einstein equation has been used as a reference to calculate the stoke radius of a solute from its diffusivity in a solution (Einstein, 1956), as follow:

$$
D_{A B}=\frac{k T}{6 \pi \eta a}
$$

where, $D_{A B}, k, \eta$, a are the diffusivity $\left(\mathrm{m}^{2} / \mathrm{s}\right)$, Boltzmann's constant, solvent viscosity and the Stokes radius $(m)$ respectively. Furthermore, the diffusivity $\left(\mathrm{D}_{\mathrm{AB}}\right)$ of solute in the membrane matrix is assumed to be equal to that in bulk solutions approximately and can also be obtained from the following equation (Singh et al., 1998); 


$$
D_{A B}=\frac{2.5 \times 10^{6} k T}{\left\{\eta(M[\eta])^{1 / 3}\right\}}
$$

where, $[\eta]$ and $M$ represent the intrinsic viscosity and molecular weight of the polymer, respectively. [ ] of a particular molecular weight of PEG can be calculated by:

$$
[\eta]=4.9 \times 10^{-4} M^{0.672}
$$

by combining both of Equation. 3.14 and Equation.3.15, the solute diameter could be expressed as:

$$
a=2.122 \times 10^{-8}(M[\eta])^{1 / 3},
$$

Thereafter, the stoke radius a $(\mathrm{cm})$ of PEG calculated by submitting the $[\eta]$ at various molecular weight PEGs, as follows:

$$
a(\mathrm{PEG})=16.73 \times 10^{-10} M^{0.557}, \quad 3.18
$$

for polyethylene oxide (PEO), the stoke radius formula can be written as;

$$
a(\mathrm{PEO})=10.44 \times 10^{-10} M^{0.587}, \quad 3.19
$$

since solute diameter $\left(\mathrm{d}_{\mathrm{s}}\right)=2 a(\mathrm{~nm})$, The Stokes-Einstein equation has been rearranged to facilitate the calculation and written as follows:

$$
d_{S}(\mathrm{PEG})=2\left(16.73 \times 10^{-10} M^{0.557}\right) \times 10^{7},
$$


From the other hand, pore size distribution represents one of the most significant characteristics of membranes and could be evaluated from a set of solute separation coefficients versus pore size calculated from retention of these solutes, and finally expressed by the probability density function using log-normal distribution. An assumption was made that sieving is the dominant mechanism through solute permeation while corrections assigned to hindered transport were taken into account (García-Martín et al., 2014; Gholami et al., 2003).

According to the log-normal probability function which reported by (Michaels, 1980), membranes sieving curves can be described accurately. Where the correlation between solute separation and solute diameter could be expressed as:

$$
f=\operatorname{erf}(z)=\frac{1}{\sqrt{2 \pi}} \int_{-\infty}^{z} e^{\frac{-u^{2}}{2}} d u
$$

where;

$$
Z=\frac{\ln d_{s}-\ln \mu_{S}}{\ln \sigma_{g}}
$$

$\mathrm{d}_{\mathrm{s}}$ referred to the solute diameter. While at $f=50 \%$ solute separation, $\mu$ s represents the mean diameter of solute. And $\sigma_{g}$ represented the standard deviation. By plotting solute separation $(f)$ (Equation 3.9) and solute diameter $\left(d_{s}\right)$ (Equation 3.20) on a logprobability paper or Sigma Plot software, a linear relationship could be obtained as illustrated in the equation below;

$$
F(f)=m\left(\ln d_{s}\right)+c
$$

Since $m$ and $c$ represent the slope and intercept of the plot. From that straight line, $\mu \mathrm{s}$ at separation factor equal to $50 \%$ could be obtained in addition to $\left(\sigma_{\mathrm{g}}\right)$ which represents the ratio between the geometric mean diameter of solute $\left(\mu_{\mathrm{s}}\right)$ at $84.13 \%$ and $50 \%$ solute separation. $\left(\sigma_{\mathrm{g}}\right)$ and $\left(\mu_{\mathrm{s}}\right)$ determine the sharpness and the position of the retention curves respectively. Instead of the geometric standard deviation about mean diameter $\left(\sigma_{\mathrm{g}}\right)$ and the mean diameter of solute $(\mu \mathrm{s})$, the geometric standard deviation 
$(\sigma p)$ and the mean pore size $\left(\mu_{\mathrm{p}}\right)$ readings are taken from the lognormal probability plot since both these parameters are considered to be same $\left(\sigma_{\mathrm{p}}=\sigma_{\mathrm{g}}, \mu_{\mathrm{p}}=\mu_{\mathrm{s}}\right)$ due to discarding the hydrodynamic and steric interactions between the pores and solute (Cooper \& Van Derveer, 1979; Michaels, 1980; Singh et al., 1998).

Finally, the probability density function can be used to describe the various pore size distribution curves by submitting the data of $\sigma_{\mathrm{p}}$ and $\mu_{\mathrm{p}}$ for each membrane (Khayet \& Matsuura, 2003; Singh et al., 1998) as follow:

$$
\frac{d f\left(d_{p}\right)}{d\left(d_{p}\right)}=\frac{1}{d_{p} \ln \sigma_{p}(2 \pi)^{\frac{1}{2}}} \exp \left(-\frac{\left(\ln d_{p}-\ln \mu_{p}\right)^{2}}{2\left(\ln \sigma_{p}\right)^{2}}\right)
$$

where $d_{p}$ is the diameter of the pore. 
Chapter Four - Investigation of Polyethersulfone polymer concentration on UF membrane characteristics and performance; a comprehensive study

\section{Chapter Four}

\section{Investigation of Polyethersulfone polymer concentration on UF membrane characteristics and performance; a comprehensive study}

\subsection{Introduction}

Membrane technology bestows significant opportunities for the future prospects of water treatment, taking into account its cost and energy efficiency when compared with conventional separation techniques. In addition, introducing the technology enables many industries to become eco-friendlier by facilitating the recycling of waste materials and resources recovery (Rohani et al., 2011). Substantial effort has been devoted in the last few decades to fabricate synthetic polymeric membranes with desired selectivity, permeability, structure and physiochemical properties. Numerous synthetic membranes structures have been produced via a variety of techniques and synthetic materials. One of the most notable techniques is the phase inversion (PI) or phase separation (PS) technique, induced by immersion precipitation, and most of the flat sheet-polymeric membranes are made using this technique (Zhao et al., 2013). Compared to conventional techniques, PI is extremely versatile and allows high flexibility in membrane material selection, and a wide range of pore sizes $(1-10,000 \mathrm{~nm})$ can be obtained, as long as the system (polymer-solvent) miscibility gap is over a defined temperature and concentration range (Hilal et al., 2015). However, during the fabrication of asymmetric polymeric membranes by PI, many major and secondary variables can also be adjusted to control the overall membrane properties. The interplay 
Chapter Four - Investigation of Polyethersulfone polymer concentration on UF membrane characteristics and performance; a comprehensive study

of all synthesis parameters influences the fabricated membrane morphology, mechanical strength, permeability, and selectivity. One of the key factors affecting membrane characteristics and performance is the host polymer concentration used to prepare the membranes.

It is well established in the literature that membrane's structure, obtained at the end of the PI process, relies on the physio-chemical characteristics of dope casting solution. As the concentration of a dope casting solution changes, surface characteristics of that synthesised polymeric membrane would vary. A range of diverse complex structures may form depending on the process conditions and solution composition. Indeed, this would be reflected at the permeation characteristics, selectivity and antifouling behaviour of these membrane. Particularly, a higher polymer concentration will induce the formation of a higher viscosity dope solution at the bimodal-phase separation point and form a denser structure.

Currently, membrane process sustainability is still doubted in some industrial applications, and fouling of membranes represents an inherent and significant issue due to its complexity and variety (Ismail et al., 2015). According to Hilal et al. (2005), membrane fouling phenomena can take place at different locations in membranes, including at both selective layer and pore walls. Internal fouling refers to the deposition and adsorption of small particles or molecules onto the pore entrances or the inside of a pore of the membrane whereas external fouling refers to the accumulation of rejected molecules upon the membrane surface. These fouling mechanisms could occur at different degrees depending on the characteristics of the membrane and targeted organic molecules. For instance, the fouling behaviour of porous microfiltration (MF) and ultrafiltration (UF) membranes are significantly different to those observed for seawater nanofiltration (NF) and reverse osmosis (RO) membranes. Pore blocking is the common fouling mechanism in the MF and UF membranes while it is not been considered for the NF and RO membranes. Moreover, pore blocking is suggested to be a more significant contributor than cake formation in UF membrane fouling, and it is influenced by the surface density of membrane pores and the pore size distribution (Bowen et al., 1996; Wang \& Tarabara, 2008). Thus, understanding the contributing fouling process mechanisms is a fundamental approach to provide better means for minimising membrane fouling and its sub-consequences, enabling the adoption of an 
Chapter Four - Investigation of Polyethersulfone polymer concentration on UF membrane characteristics and performance; a comprehensive study

adequate membrane separation process and operating conditions for targeted industrial applications.

Organic compounds such as humic substances, proteins, and polysaccharides in water are the most abundant foulants in UF of many industrial operations. These foulants are inherently severe for pressure-driven membranes due to their strong irreversible adsorption on the surface of the membrane, causing a dramatic decline in membrane permeation, and raise many issues regarding product quality and process cost (Ang et al., 2015). The degree of selectivity and fouling does not only depend on membrane properties but also on the complexity of the foulants' composition, their molecular weight and the charge of organic compounds, alongside the hydrodynamic conditions and solution chemistry (Lim \& Mohammad, 2010). Solution chemistry is critical for controlling the charge and configuration of organic foulants that will influence the intramolecular and molecules-membrane interactions, and hence membrane performance (Zazouli et al., 2010). More precisely, performance may differ for different solute types (charged or neutral) and shapes (globular, linear or branched solute), that have similar molecular weight (Mulder, 1996). This may be the case even under the same operating conditions

In this chapter, an investigation was made into the influence of PES concentration on UF membrane morphology and surface characteristics. PES/UF membranes have been synthesised with a wide range of structures that correspond to typical commercially available UF membranes. Fouling behaviour and retention efficiency tests were conducted to give a more precise and comprehensive assessment of UF membrane fouling. Three different organic model foulants (HA, BSA, and NaAlg) have been applied for this comprehensive fouling assessment, under a broad range of feed concentration and solution $\mathrm{pH}$.

\subsection{Membrane fabrication}

Since a very low and high casting solution viscosity results in a brittle and tight membrane respectively, four asymmetric PES flat sheet membranes, with a different PES polymer weight percent (wt \%), were fabricated according to the procedure described in 3.3.1 (Chapter 3). Each membrane was denoted based on the membrane composition, as tabulated in Table 4.1 . 
Chapter Four - Investigation of Polyethersulfone polymer concentration on UF membrane characteristics and performance; a comprehensive study

Table 4.1: Composition of PES membranes.

\begin{tabular}{llll}
\hline Membrane ID & PES wt\% & NMP wt\% & PVP K30 wt\% \\
\hline PES22 & 22 & 76 & 2 \\
PES20 & 20 & 78 & 2 \\
PES18 & 18 & 80 & 2 \\
PES16 & 16 & 82 & 2 \\
\hline
\end{tabular}

\subsection{Polymeric membranes characterisation}

\subsubsection{Cross-sectional analysis by scanning electron microscope (SEM)}

The cross-sectional structure has a significant influence on a membrane's transport mechanism. SEM images of all membranes obtained from 16, 18, 20 and $22 \mathrm{wt} \%$ PES casting solutions show a typical asymmetric structure with clear active and supporting layer for all membranes (Figure 4.1).

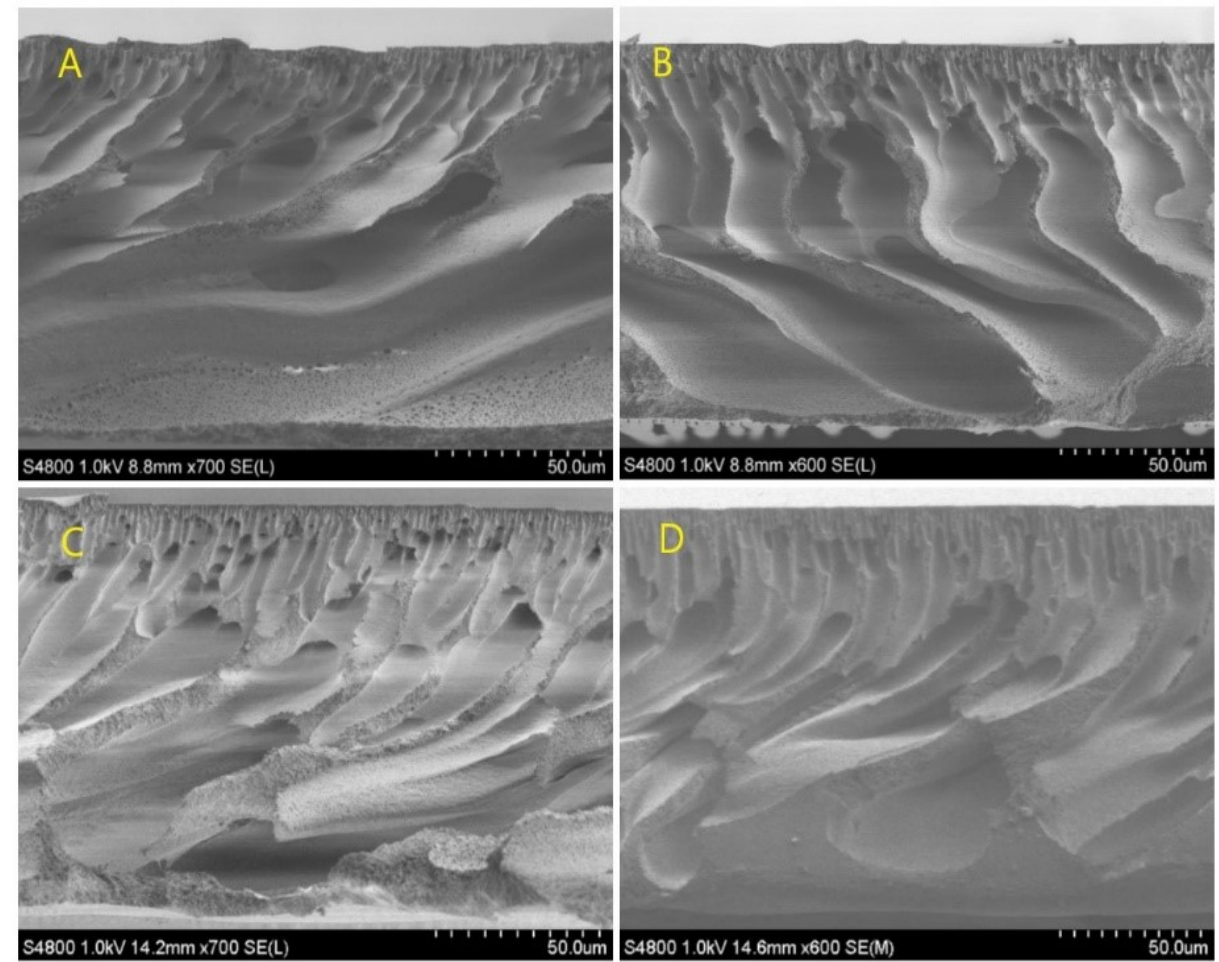

Figure 4.1: SEM cross-section images for; (A) PES16, (B) PES18, (C) PES20, and (D) PES22 membrane.

Figure 4.1A shows a well-developed skin layer, connected and supported by a system of wide finger-like macro-pores, as can be observed at the bottom of the PES16 
Chapter Four - Investigation of Polyethersulfone polymer concentration on UF membrane characteristics and performance; a comprehensive study

membrane. This structure is most apparent for membranes fabricated with a low viscosity casting solution, where the nonsolvent exchange rate into the polymer lean phase exceeds the outward solvent diffusion rate (Sofiah et al., 2010). Increasing the polymer concentration suppressed these macro-pores in the sub-layer and led to a slightly narrower finger-like structure from the top to the bottom layer of the PES18 membrane, (Figure 4.1B). While PES20 and PES22 membranes, which had the highest polymer concentration, manifested the densest structure, in comparison to PES18 and PES16. This was correlated with their higher casting solution viscosity, leading to a denser membrane structure. A higher viscosity solution can hinder the solventnonsolvent exchange rate at the interface between the surface and the nonsolvent, producing a dense skin layer and less porous membrane (Rahimpour et al., 2012). Indeed, the microporous finger-like structure observed in (Figure 4.1C and Figure 4.1D), are supported on a sponge-like structure at the bottom of the membranes, especially for PES22.

\subsubsection{Surface morphology analysis by atomic force microscope (AFM)}

AFM was used to characterise the membrane surfaces, and Figure 4.2 presents the 1 $\mu \mathrm{m} \times 1 \mu \mathrm{m} 3 \mathrm{D}$ images that have been used for membrane roughness analysis. All membranes manifested a smooth surface, which is the main surface characteristic associated with PES membranes. The relationship between the PES concentration used to prepare the membranes, and the membrane roughness parameters are listed in Table 4.2. The presented parameters demonstrate that as the polymer wt $\%$ decreases, a rougher membrane surface can be formed. The PES22 membrane, which was fabricated with the highest concentration, had the flattest membrane surface with only $19.39 \mathrm{~nm}$ difference between the highest peak and lowest valley. While the $R_{m s}$ value was 2.28 $\mathrm{nm}$. A decrease in polymer concentration (PES20 membrane), induced slightly higher surface roughness parameters. The lowest PES wt \% dope solutions (PES16) resulted in the roughest surface and highest peak to valley values, $4.54 \mathrm{~nm}$, and $40.18 \mathrm{~nm}$, respectively. In similar research, a smoother surface topography was visualised by Rahimpour et al., in addition to smaller pore size, fewer porous sub-layer, and lesser finger-like pores when PES concentration increased from $14.4 \mathrm{wt} \%$ to $16 \mathrm{wt} \%$ (Rahimpour et al., 2009). 
Chapter Four - Investigation of Polyethersulfone polymer concentration on UF membrane characteristics and performance; a comprehensive study
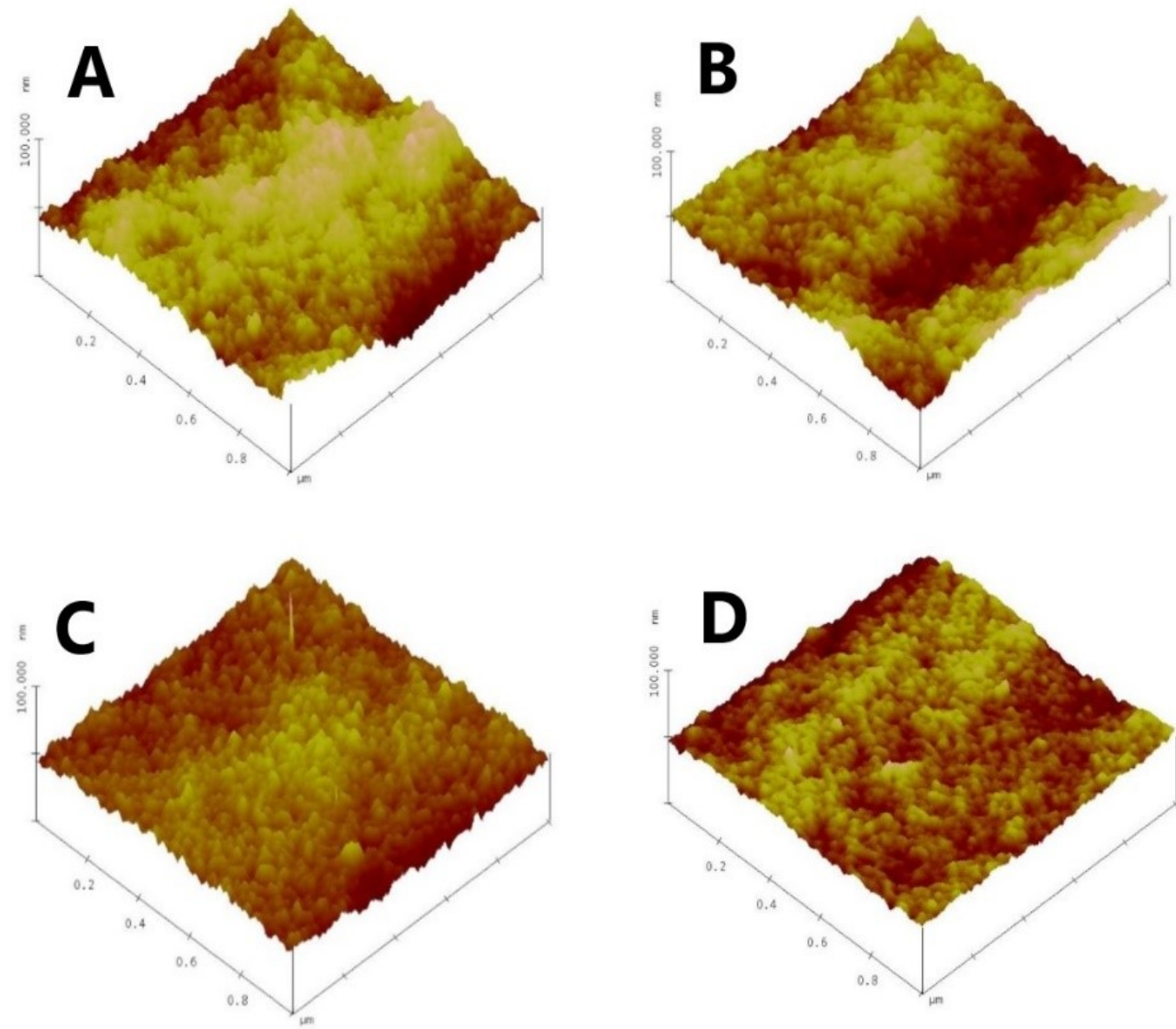

Figure 4.2: (1 $\mu \mathrm{m} \times 1 \mu \mathrm{m})$ AFM images for; (A) PES16, (B) PES18, (C) PES20 and (D) PES22 membrane.

Table 4.2: Surface roughness parameters of the membranes.

\begin{tabular}{lllll}
\hline Membrane & Polymer wt.\% & $\mathrm{R}_{\mathrm{a}}(\mathrm{nm})$ & $\mathrm{R}_{\mathrm{ms}}(\mathrm{nm})$ & $\mathrm{R}_{\max }$ \\
\hline PES22 & 22 & 1.81 & 2.28 & 19.39 \\
PES20 & 20 & 3.26 & 4.12 & 56.58 \\
PES18 & 18 & 3.79 & 4.77 & 32.09 \\
PES16 & 16 & 4.54 & 5.64 & 40.18 \\
\hline
\end{tabular}

\subsubsection{Solute transport method}

Methods based on rejection data of solutes are proven to be more indicative methods for characterisation of membrane separation performance (Kang et al., 2014). In this work, the solute transport method has been applied to estimate the mean pore size of the UF membranes, while the lognormal distribution curves were used to describe the 
Chapter Four - Investigation of Polyethersulfone polymer concentration on UF membrane characteristics and performance; a comprehensive study

pore size distribution, more detailed description of the procedure was given in section 3.3.5 (chapter 3). The influence of PES polymer concentration on the pore size and pore size distribution of membranes was determined from the PEG/PEO solution separation curves for each membrane (Figure 4.3 ) and the data for geometric mean pore size $\left(\mu_{\mathrm{p}}\right)$ and their geometric deviation $\left(\sigma_{\mathrm{p}}\right)$ are tabulated in Table 4.3. While Figure 4.4 presents pore size distribution curves for the membranes. The data in Table 4.3 clearly demonstrates that diminishing the polymer wt.\% has given rise to a bigger pore size along with a wider pore size distribution range. PES22 revealed the smallest mean pore diameter $(2.17 \mathrm{~nm})$ compared to the other membranes, and mean pore diameter gradually increased, with a decrease in PES concentration, from $2.93 \mathrm{~nm}, 4.41 \mathrm{~nm}$ and $10.79 \mathrm{~nm}$ for PES20, PES18, and PES16, respectively. Geometric standard deviation values were comparable and reasonable for all UF membranes. Values of $\sigma_{\mathrm{p}}$ associated with their corresponding $\mu$ p values ranged from 1.39 to 1.89 .

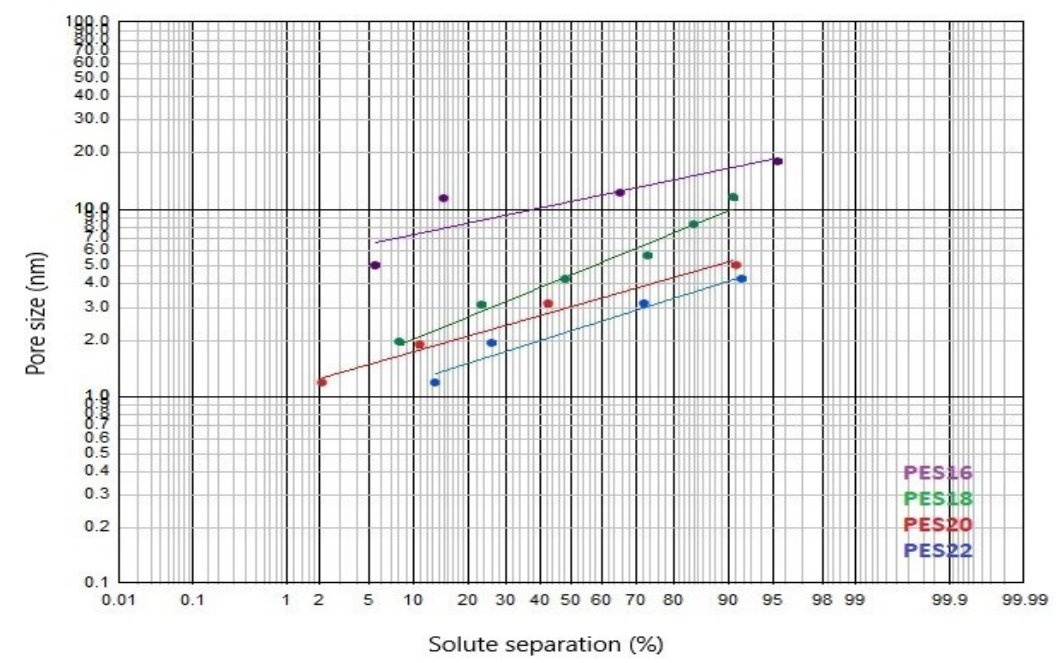

Figure 4.3: Solute separation curve plotted on log-normal probability paper (LN3 software).

Table 4.3: Geometric mean pore size, standard deviation and MWCO of membranes.

\begin{tabular}{lllll}
\hline Membrane ID & Polymer wt.\% & $\mu_{\mathrm{p}}(\mathrm{nm})$ & $\sigma_{p}$ & MWCO \\
\hline PES22 & 22 & 2.177 & 1.67 & $\sim 6 \mathrm{kDa}$ \\
PES20 & 20 & 2.935 & 1.54 & $\sim 10 \mathrm{kDa}$ \\
PES18 & 18 & 4.41 & 1.89 & $\sim 35 \mathrm{kDa}$ \\
PES16 & 16 & 10.799 & 1.39 & $\sim 100 \mathrm{kDa}$ \\
\hline
\end{tabular}


Chapter Four - Investigation of Polyethersulfone polymer concentration on UF membrane characteristics and performance; a comprehensive study

The changes in the pore size distribution curves (Figure 4.4) correlate with the pore size trend. The curves shifted to the right as the polymer weight percent was reduced for PES22 to that of PES20, which had a slightly wider distribution for the pores but with a decrease in their density. While PES18 and PES16 curves had pointedly higher shifts to the right demonstrating wider pore size distributions compared to other membranes, in addition to a lower mean pore density function. Membranes with $18 \mathrm{wt}$ $\%$ polymer or higher tended to produce a sharp cut-off in pore size while less than this critical wt \% value showed a wider pore size distribution and a diffuse cut-off. These results clearly demonstrate that the polymer wt $\%$ in the casting solution has a significant influence on the fabricated membrane's permeation characteristics.

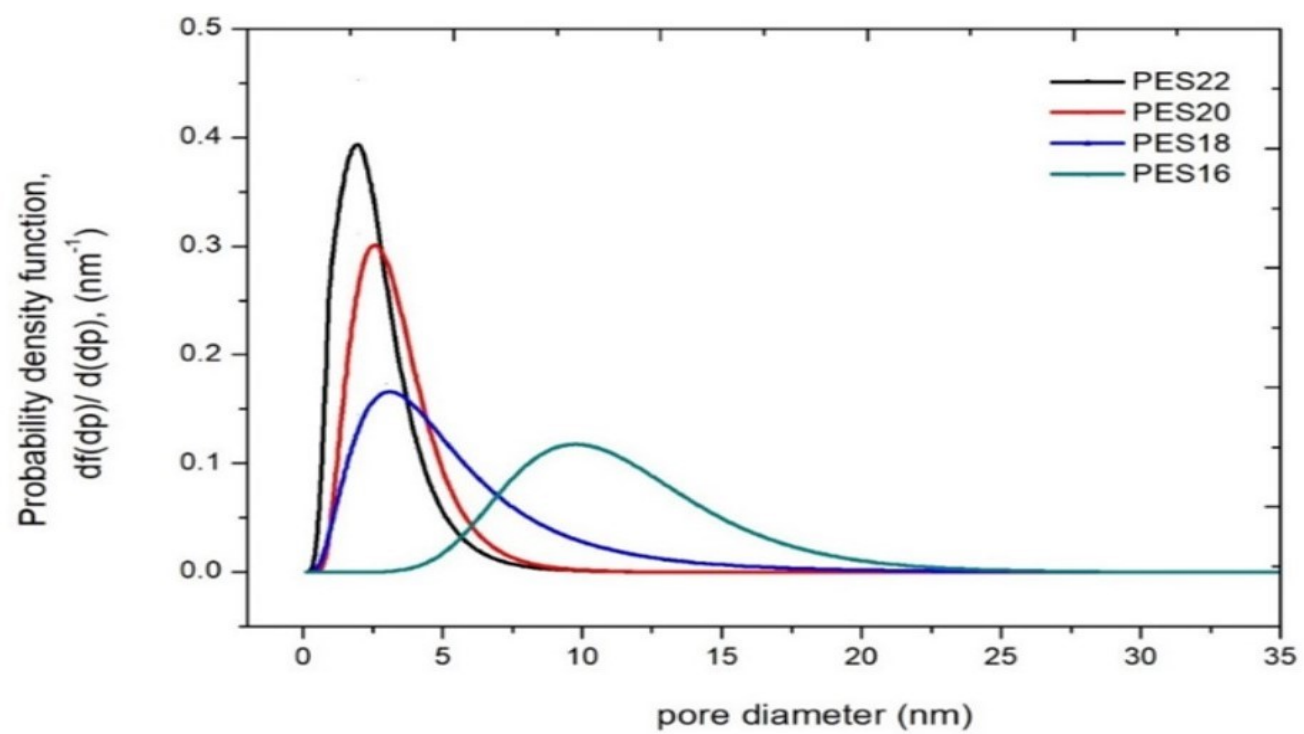

Figure 4.4: Probability density function curves of UF/PES membranes

\subsubsection{Zeta potential of membranes}

Zeta potential (ZP) is known as an effective tool that can provide fundamental insights into membrane ageing, separation mechanisms, membrane fouling, and cleaning. To investigate the influence of varying PES concentration on the membrane surface charge characteristics, and to specify the isoelectric point (IEP) of the fabricated membranes. Herein, a novel methodology employing a zeta potential planar cell (ZEN1020) in conjunction with laser doppler electrophoreses (LDE) has been applied to characterize the ZP of PES membranes, an extended description is presented elsewhere (Thomas et $a l ., 2017)$. The measurements were then compared with the well know TSP to evaluated the reliability of the new technique. Figure 4.5A presents a typical phase distribution, 
Chapter Four - Investigation of Polyethersulfone polymer concentration on UF membrane characteristics and performance; a comprehensive study

where all bands for each of the four displacements are distinct, tightly grouped, with minimal noise, obtained for the PES16 membrane at $10 \mathrm{mM} \mathrm{NaCl}$ and pH8.4. While Figure $4.5 \mathrm{~B}$ presents the negatively charged tracer particles electrophoretic mobility measured at four different distances from the membrane's surface and showing a high regression correlation $\left(\mathrm{R}^{2}=0.961\right)$ for the intercept of the displacement plot. A good phase plot is an indication of the data measurements reliability while indistinguishable noisy bands can be produced when data have influenced by the insufficient transition of laser light entering the sample.
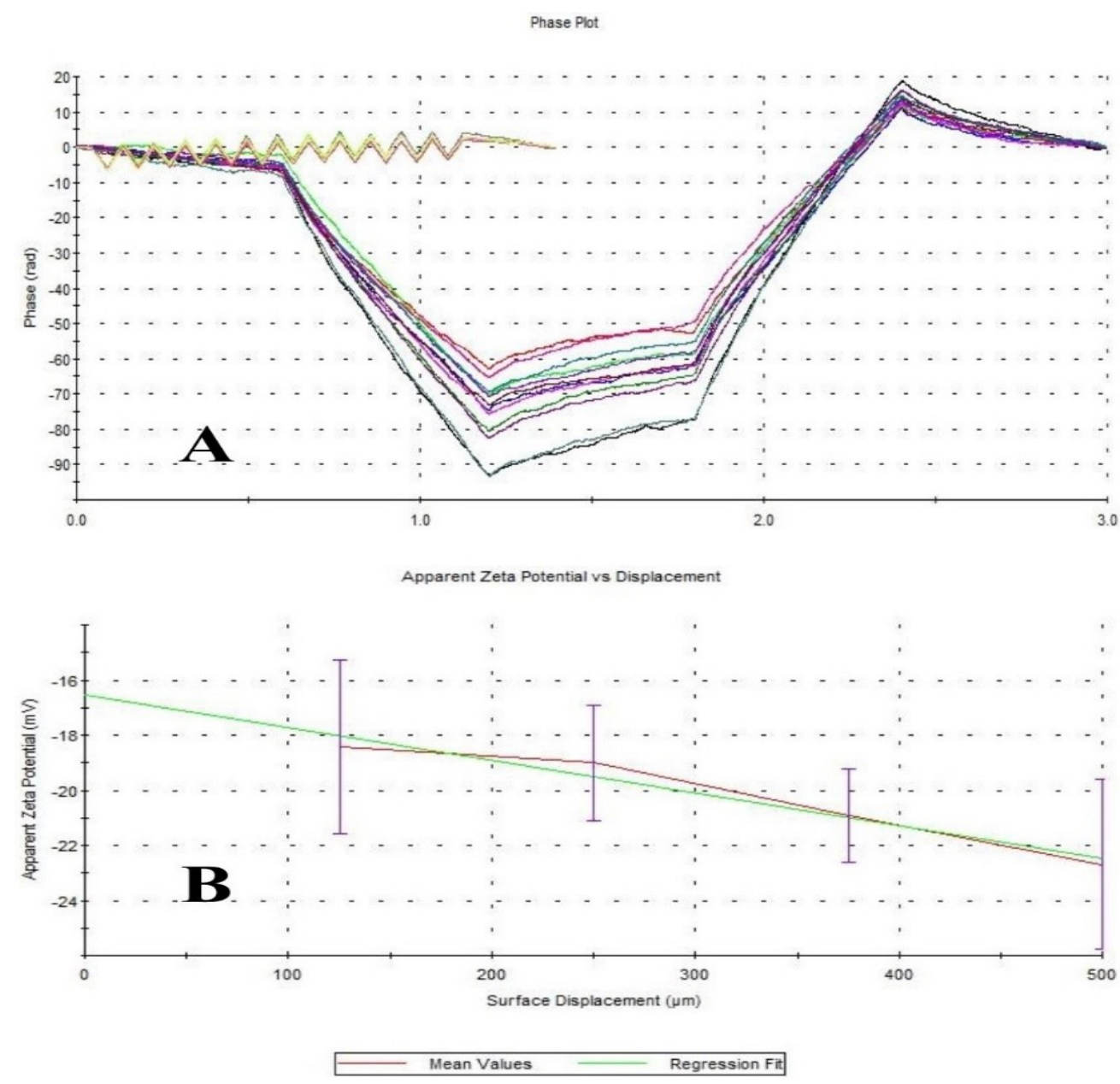

Figure 4.5: (A) Measured electrophoretic mobility of tracer particles as a function of displacement from the membrane surface and (B) phase plots at four different displacements from the membrane surface.

It should be noted here that the electro-osmotic flow at the sample surface will tend to fall off with increasing distance hence; close to the surface, the tracer mobility will be dominated by the electro-osmotic surface flow, while at distances further from the surface it will be dominated by the electrophoretic motion of the tracer itself. 
Chapter Four - Investigation of Polyethersulfone polymer concentration on UF membrane characteristics and performance; a comprehensive study

All PES membranes were found to be negatively charged under the entire $\mathrm{pH}$ range investigated, and the absolute values of $\mathrm{ZP}$ decreased with lower $\mathrm{pH}$ values for all UF membranes. Furthermore, no IEP could be identified, as shown in Figure 4.6. The size of ZP obtained from characterising the bare PES membranes is in line with previous research (Liu et al., 2015). Moreover, when the host polymer concentration in the casting solution was increased, a clear pattern was evident, with an upward shift in the $\mathrm{ZP}$ curves. For instance, at $\mathrm{pH} 6$ and $10 \mathrm{mM} \mathrm{NaCl}$ ionic strength, the smallest PES membrane concentration (PES16) had a slightly higher zeta potential value $(-7.78 \mathrm{mV})$ than other higher concentration membranes, which were found to have a ZP of -6.5 $\mathrm{mV},-6.81 \mathrm{mV}$ and $-5.4 \mathrm{mV}$ for PES18, PES20 and PES22, respectively. The higher ZP measured for the membrane fabricated with a lower PES concentration could be due to the higher surface area resulting from the higher surface roughness of PES16 membranes (Table 4.2). The increased surface area will give rise to more functional groups on the surface of these PES membranes, which will contribute to the total net charge. The ZP measurement patterns, as a function of $\mathrm{pH}$, obtained in this work were contradictory with what was observed in previous research by (Sofiah et al., 2010) who reported an increase in the ZP negativity of PES membrane by almost double as the polymer concentration increased in the dope casting solution from $13 \mathrm{wt} \%$ to $17 \mathrm{wt} \%$.

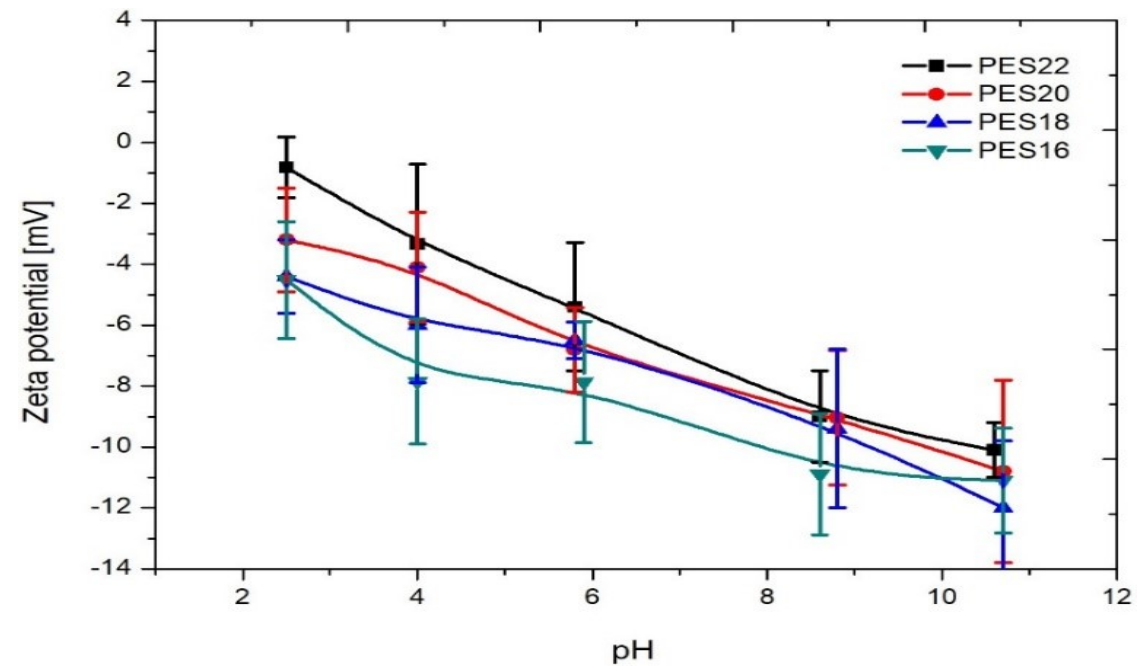

Figure 4.6: Surface zeta potential profile for the UF membranes as a function of $\mathrm{pH}$, determined by LDE.

Tangential streaming potential (TSP) measurements are known as the most commonly used technique to evaluate membranes zeta potential (Hanafi et al., 2016). 
Chapter Four - Investigation of Polyethersulfone polymer concentration on UF membrane characteristics and performance; a comprehensive study

The TSP measurements have been carried out, as illustrated in (section 3.2.4.1), to compare the ZP of membranes with those acquired by LDE in order to gain a better understanding to the precision, accuracy, and reproducibility of the former technique. Similarly, all PES membranes have revealed negatively charged surfaces under the entire $\mathrm{pH}$ range performed, Figure 4.7. ZP of PES membranes obtained from the TSP were congruent with those of LDE mentioned earlier in Figure 4.6. A clear upward shift trend in the zeta potential curves was presented with increasing the host polymer concentration in the casting solution. At $6.5 \mathrm{pH}$, the lowest concentration PES16 membrane has exhibited a greater zeta value $(-9.88 \mathrm{mV})$ than other membranes which determined to be following the order; $(-8.17 \mathrm{mV})$ PES18 $>(-6.53 \mathrm{mV})$ PES20 $>$ and $(-5.96)$ PES22. The results obtained from TSP were of similar magnitude and trend in comparison to results obtained from LDE measurements, except higher results uncertainty were associated with the LDE method.

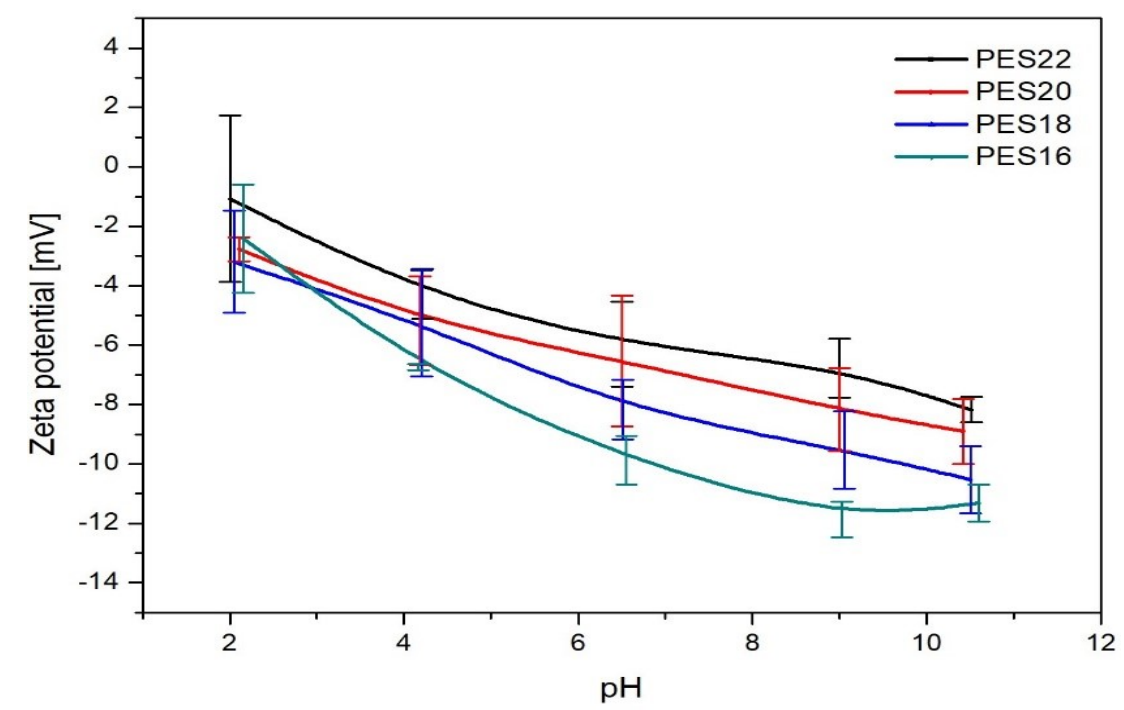

Figure 4.7: Surface zeta potential profile for the UF membranes as a function of $\mathrm{pH}$, determined by TSP.

\subsubsection{Mechanical strength}

The mechanical robustness of membranes can be used as an indicator to predict their durability and reliability under stressed conditions during industrial operations. Higher mechanical strength implies extended membrane lifespan and resistance to cleaning regimes (Powell et al., 2017). Determining the mechanical characteristic of material requires measuring its responses to the applied force. One common method to achieve that is through straining the material sample under controllable strain rate and carefully 
Chapter Four - Investigation of Polyethersulfone polymer concentration on UF membrane characteristics and performance; a comprehensive study

measuring the corresponding required load. Indeed, strain-stress behaviour can be developed from the displacements and measured forces and the sample initial dimension (Vinci \& Vlassak, 1996).

Mechanical properties of synthesised membranes prepared with different concentrations of PES were tested according to the procedure described in section 3.2.3. Figure 4.8, presents typical stress/strain curves for the synthesised membranes. All curves had two distinctive portions comprising of an initial linear followed by a curved region that corresponded to the elastic response and inelastic response before the yield at maximum stress, respectively. The elastic modulus (Young's modulus) was calculated from the slope values of the initial linear portions in the stress/strain graph. Higher values of tensile strength at break, elongation and Young's modulus values were associated with the membrane fabricated from the highest PES concentration (PES22), and these values decreased with the decrease in the concentration of polymer used in membrane fabrication, as summarised in Table 4.4. Increasing the membrane casting solution concentration to $22 \mathrm{wt} . \%$ led to increasing both maximum tensile strength and elongation $\%$ by almost double their values as compared to that of $16 \mathrm{wt} . \%$, while the elastic modulus was improved by 1.5 fold. Similar observations were obtained by (AlMalek, 2012), when the PES increased from 15 to $20 \mathrm{wt} \%$, the tensile strength improved from 4.1 Mpa to 7.78 Mpa. The different mechanical properties of membranes are due to structural changes caused by higher viscosity casting solution, and the disappearance of big macrovoids at the bottom of membranes that are replaced by the formation of narrower finger-like structures, as discussed earlier (Section 4.3.1, Figure 4.1).

Table 4.4: Mechanical characteristics of UF membranes

\begin{tabular}{lllll}
\hline Membrane ID & Polymer wt.\% & $\begin{array}{l}\text { Tensile strength } \\
(\mathrm{MPa})\end{array}$ & Elongation [\%] & $\begin{array}{l}\text { Young's } \\
\text { modulus }\end{array}$ \\
\hline PES22 & 22 & 6.358 & 39.5467 & 110.84 \\
PES20 & 20 & 5.391 & 35.7333 & 100.12 \\
PES18 & 18 & 4.394 & 27.2 & 90.596 \\
PES16 & 16 & 3.372 & 22.2 & 73.85 \\
\hline
\end{tabular}


Chapter Four - Investigation of Polyethersulfone polymer concentration on UF membrane characteristics and performance; a comprehensive study

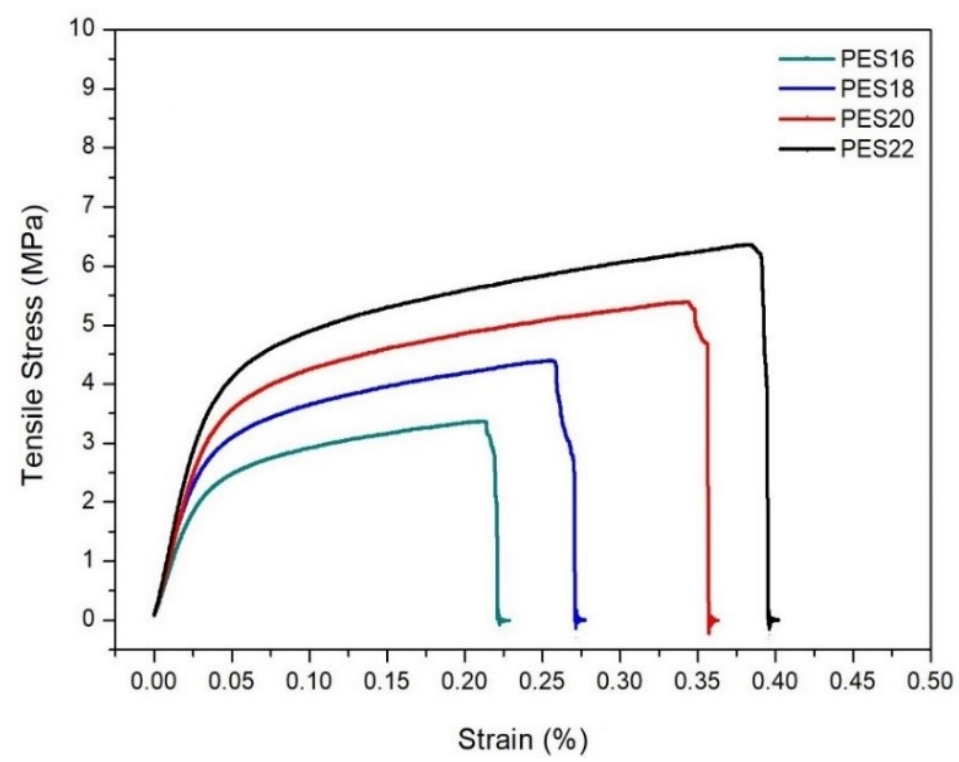

Figure 4.8: Influence of dope casting solution concentration on strain-stress curves relationship of membranes.

\subsubsection{Pure water flux and hydrophilicity/hydrophobicity of membranes}

A distinct inverse correlation has been unsurprisingly observed between the hydraulic permeability and polymer concentration of fabricated membranes (Figure 4.9). Increasing the PES polymer concentration from $16 \mathrm{wt} . \%$ to $18 \mathrm{wt} . \%$ led to a decrease in PWF of membranes from $560 \pm 301 / \mathrm{m}^{2} . h$ to $172 \pm 121 / \mathrm{m}^{2} . \mathrm{h}$, respectively. Further increase in the concentration gave rise to a further decrease in the PWF value to $48 \pm 4$ $1 / \mathrm{m}^{2} . \mathrm{h}$, for the PES20 membrane. While the highest PWF decline to $12 \pm 0.81 / \mathrm{m}^{2} . \mathrm{h}$ was associated with the highest polymer concentration (22 wt \%), for the membranes synthesised in this work.

This difference in PWF values between membranes was due to the different casting solution viscosities used to prepare the membranes. A higher casting solution viscosity/concentration diminishes the diffusional exchange rate during the phase inversion between the solvent and nonsolvent, where a denser skin layer with lower porosity will be formed, due to the delayed demixing. This will ultimately result in lower hydrodynamic permeability of the membranes synthesised with a higher polymer concentration. 
Chapter Four - Investigation of Polyethersulfone polymer concentration on UF membrane characteristics and performance; a comprehensive study

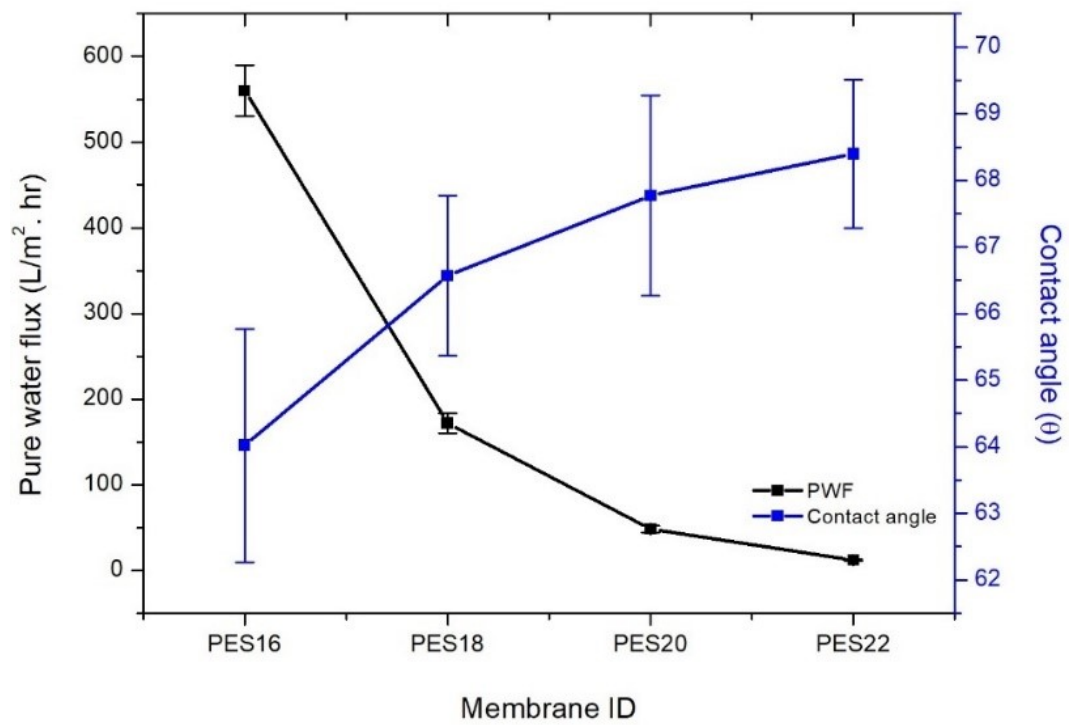

Figure 4.9: PWF and contact angle measurements for membranes.

All membranes had similar hydrophobicity as measured by contact angles (CA) (within the sixties), which were in the range of bare polyethersulfone membranes reported in the literature (Rahimpour et al., 2010). Only a slight decrease was observed as the polymer wt.\% decreased even though all membranes were prepared with identical conditions and materials except for the polymer concentration (Figure 4.9). This could be ascribed to differences in other surface characteristics of the membranes, e.g. roughness parameters, pore size, and pore size distribution since CA values could be influenced by any change in these surface characteristics (Rana \& Matsuura, 2010). These results were in agreement with the previously reported literature by (Balta et al., $2012)$, and observed a gradual decrease in the contact angle values $\left(73^{\circ}\right.$ to $\left.48^{\circ}\right)$ when the PES casting solution concentration decreased from $32-25 \mathrm{wt} \%$.

\subsection{Evaluation of membranes performance with organic foulants}

A lab-scale cross flow apparatus (Figure 3.23) was employed to investigate the UF membranes organics retention efficiency and fouling behaviour over short filtration duration $(120 \mathrm{~min})$. The experiments with the chosen representative model organic (HA, BSA and NaAlg) foulants were performed at three different initial feed concentrations (20,60 and $100 \mathrm{ppm})$ and $\mathrm{pH}(4,7$ and 10). 
Chapter Four - Investigation of Polyethersulfone polymer concentration on UF membrane characteristics and performance; a comprehensive study

The influence of natural organic material (NOM) on membrane performance was investigated using HA as a model macromolecule. Experimental data showing the influence of HA solution $\mathrm{pH}$ on the retention coefficient and fouling behaviour of the four neat PES membranes are presented in Figure 4.10. As apparent in Figure 4.10A, all UF membranes manifested higher than $90 \%$ retention for HA over the entire $\mathrm{pH}$ range tested. HA is of natural origin and not of uniform composition. According to the manufacturer, it consists of heteropolycondensates of MW's ranging from 2 to $500 \mathrm{kDa}$, but mainly $\sim 20-50 \mathrm{kDa}$. Therefore, a true structure cannot be given and retention data for the MWCO membranes used is indicative of the presence of low molecular weight components in the Aldrich HA. Meanwhile, the observed removal coefficients were found to be at their maximum value at neutral $(\mathrm{pH} 7)$ conditions for all membranes. Under highly alkaline ( $\mathrm{pH} 10)$ feed solution, a slight decrease in the membrane retention was observed, and it was unsurprisingly found to be at the lowest value under acidic conditions (pH4). This agreed with the previous researches on the influence of the initial feed solution $\mathrm{pH}$ on the retention characteristics of membranes that suggested a difference in the size and configuration of $\mathrm{HA}$ at dissimilar $\mathrm{pH}$ conditions. A possible explanation is that a high acidic condition, induces more carboxylic groups of organic HA to be protonated, resulting in a reduction of both their macromolecular size and charge density. So, they pass more readily through membrane pores in the presence of the minimum electrostatic repulsion between membrane surfaces and organics, thus lowering the membrane rejection coefficient (Hong \& Elimelech, 1997). Interestingly, in contradiction to the trend observed in the literature, membranes with greater MWCO (PES16, PES18, and PES20), exhibited slightly higher retention compared to that of the PES22 membrane. This could be explained by the difference in membranes surface characteristics that influenced the fouling behaviour of membranes to different extents. As shown in (Figure 4.10B and Figure 4.10C), no appreciable filtrate flux decline was detected for the low cut-off UF membranes (PES22 and PES20) under the entire pH range. This was expected since the concentration of the feed $(20 \mathrm{ppm})$ and/or the short filtration time (120 min) used in the measurements was insufficient to easily foul such tight membranes under cross-flow conditions. Moreover, pore blocking and/or adsorption was insignificant due to the pore size distribution compared with the molecular cut-off distribution of the Aldrich HA. (Nyström et al., 1996) also reported no flux decline when examining the fouling behaviour of a $50 \mathrm{kDa}$ polysulfone 
Chapter Four - Investigation of Polyethersulfone polymer concentration on UF membrane characteristics and performance; a comprehensive study

membrane using the same Aldrich HA. Cho et al. claimed that for natural organic material fouling could be neglected for NF and low MWCO UF membrane, and the influence of concentration polarisation was only significant for higher than $10 \mathrm{kDa}$ MWCO membranes (Cho et al., 1999). In contrast, a slight increase in the flux was observed at neutral condition, which could be ascribed to the increased hydrophilicity of the membrane surface imparted by binding of a proportion of the hydrophobic fraction of HA on the membrane surface, leaving the hydrophilic fraction of HA directed towards the feed solution; the fouled membrane surface possessed more hydrophilicity and negativity (Mänttäri et al., 2000). In the present research, the relative flux decline was more significant for the PES18 membrane, especially at acidic conditions. However, a drastically reduced relative flux was associated with the bigger pore size membrane (PES16), (Figure 4.10D and Figure 4.10E). This discrepancy in fouling behaviour at the different membrane structures belongs to the different mechanisms contributing to the total fouling of the membranes. For instance, the initial rapid irreversible fouling that took place at the PES16 membrane was mainly caused by adsorption of HA species inside the membrane pores and between the ridge-valley structure of the surface. Progressively, these adsorbed HA molecules act as initiation sites for further HA deposition to develop the cake layer (Shi et al., 2011). The hydrophobic PES16 membrane had a high pore size distribution range corresponding to a significant size fraction of the HA. Thus a spontaneous complete pore blocking and/or narrowing mechanisms may have produced a smaller pore size distribution, which may explain the slightly higher retention of the PES16 as compared to that of the PES22 membrane. A similar conclusion was reached by, W. Yuan and AL Zydney who identified that the HA retention coefficient of a membrane with a pore size of 0.16 $\mu \mathrm{m}$ became greater than the retention obtained with membranes with much smaller 100 $\mathrm{kDa}$ and $300 \mathrm{kD}$ pores, after about 50 min of filtration (Yuan \& Zydney, 2000). 
Chapter Four - Investigation of Polyethersulfone polymer concentration on UF membrane characteristics and performance; a comprehensive study
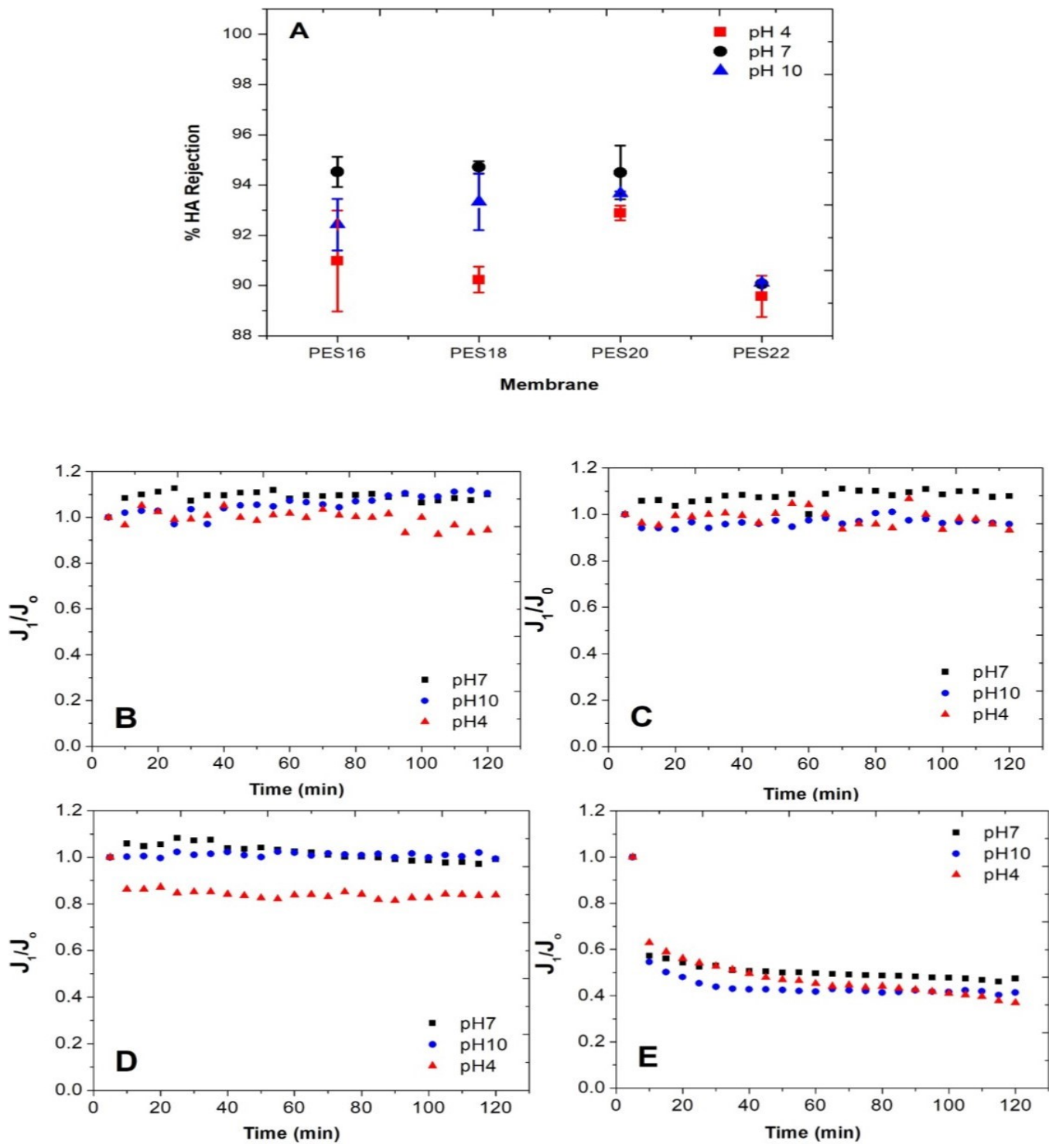

Figure 4.10: Influence of solution $\mathrm{pH}$ on HA rejection coefficients (A), and fouling behaviour of (B) PES22, (C) PES20, (D) PES18, and (E) PES16 membrane.

The influence of HA initial feed concentration on the rejection coefficients has indicated a slight increase in removal efficiency of the membranes as the HA initial feed concentration increases from 20 to $100 \mathrm{ppm}$, Figure 4.11A. These coefficients were comparable in size (within 94-96\%) except that of the $6 \mathrm{kDa}$ membrane (PES22), which manifested a slightly lower value than other membranes, mainly at the lower feed concentration $(20 \mathrm{ppm})$. When the initial feed concentration increased the influence of reversible concentration polarisation was becoming more obvious as demonstrated by the improved retentive coefficients of the PES22 membrane at high concentrations. Yuan and Zydney claimed that a highly concentrated HA feed solution 
Chapter Four - Investigation of Polyethersulfone polymer concentration on UF membrane characteristics and performance; a comprehensive study

induces a considerable concentration polarisation to take place at membranes as a consequence of the concentration-dependence of the osmotic pressure (Yuan \& Zydney, 2000). The magnitude of the relative flux patterns of the membranes is presented in (Figure 4.11B-Figure 4.11E). The PES16 membrane experienced a further flux decline, mainly when the higher (60 and $100 \mathrm{ppm})$ initial feed concentrations were introduced. This could be due to thicker cake layer formation at the surface, which reduces the permeability characteristics of membranes and enhances their retention.
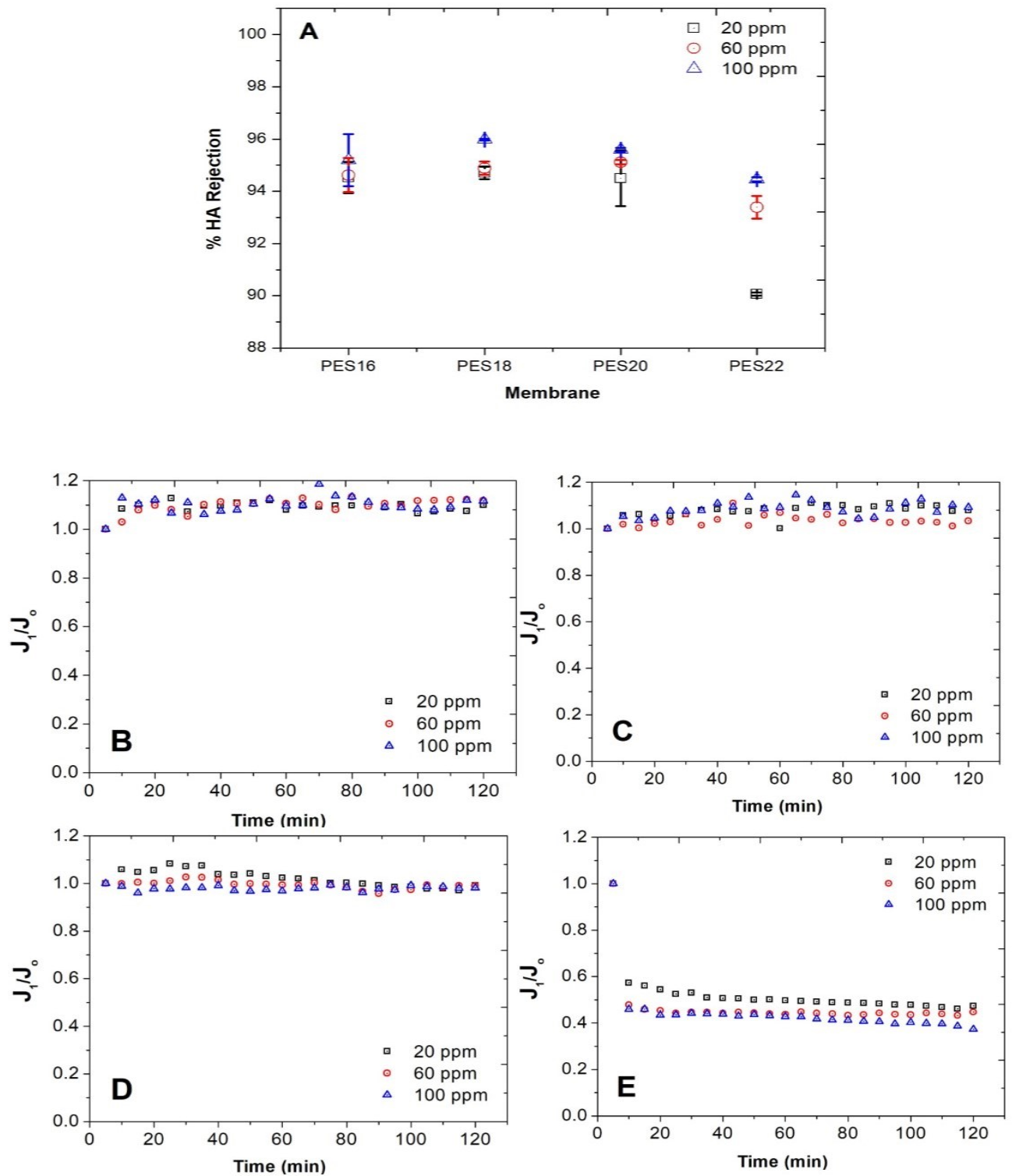

Figure 4.11: Influence of solution initial feed concentration on HA rejection coefficients (A), and fouling behaviour of (B) PES22, (C) PES20, (D) PES18, and (E) PES16 membrane. 
Chapter Four - Investigation of Polyethersulfone polymer concentration on UF membrane characteristics and performance; a comprehensive study

The assessment of the UF membranes performance against an organic polysaccharide model was carried out using individual NaAlg solution. Figure 4.12A clearly shows the removal coefficients of membranes for different feed solution chemistry $(\mathrm{pH})$ of NaAlg. Membranes behaved similarly to the trend observed for the NOM filtration, but with greater rejection magnitudes. These membranes retention coefficients were maintained as high as $\sim 96-100 \%$, with the smallest retentions ascribed to the PES22 membrane. On the other hand, the relative flux curves presented in (Figure 4.12B and Figure 4.12C) indicated that no perceivable flux decline was associated with NaAlg filtration for both PES22 and PES20 membranes. This was attributed to the insufficient feed concentration and filtration time adopted to foul these membranes; also observed for the HA filtration tests earlier in this section. In addition, the NaAlg had a greater molar mass distribution that was unable to penetrate such low cut-off membranes. For the higher MWCO PES18 membrane, a slight flux decline can be observed at the initial filtration stage suggesting that pore blocking and narrowing occurred. This was followed by a progressively increased decline without any sign of reaching the quasi-steady-state flux, Figure 4.12D. The PES16 membrane manifested a prompt and a severe relative flux decline mainly at acidic conditions, Figure 4.12E. The lower retention and the higher flux decline magnitudes, observed at low $\mathrm{pH}$, are suggested to be induced by the solution $\mathrm{pH}$ and its influence on the molecular size of the polysaccharide, as acidic conditions can influence not only the structure of NaAlg molecules but also their electrostatic repulsion with the membrane surface (Susanto et al., 2008). The lower intramolecular electrostatic interactions resulting from the protonation of the carboxylic groups of the alginates would produce smaller sized NaAlg molecules (Rhee \& Steinbuchel, 2005). Indeed, this would facilitate the passage of the resized molecules through the membrane pores and encourage their tendency to form more aggregates at low $\mathrm{pH}$. The observed relative flux of the UF membranes with individual NaAlg solutions was more detrimental than the HA fouling under identical experimental conditions and was strongly dependent on the membranes cut-off used. This was attributed to the gel-forming nature of alginate that has a greater intermolecular adhesion of the NaAlg-gel network along with their larger molecular size compared to the HA might also induce the formation of a thicker fouling layer (Zazouli et al., 2010). 
Chapter Four - Investigation of Polyethersulfone polymer concentration on UF membrane characteristics and performance; a comprehensive study
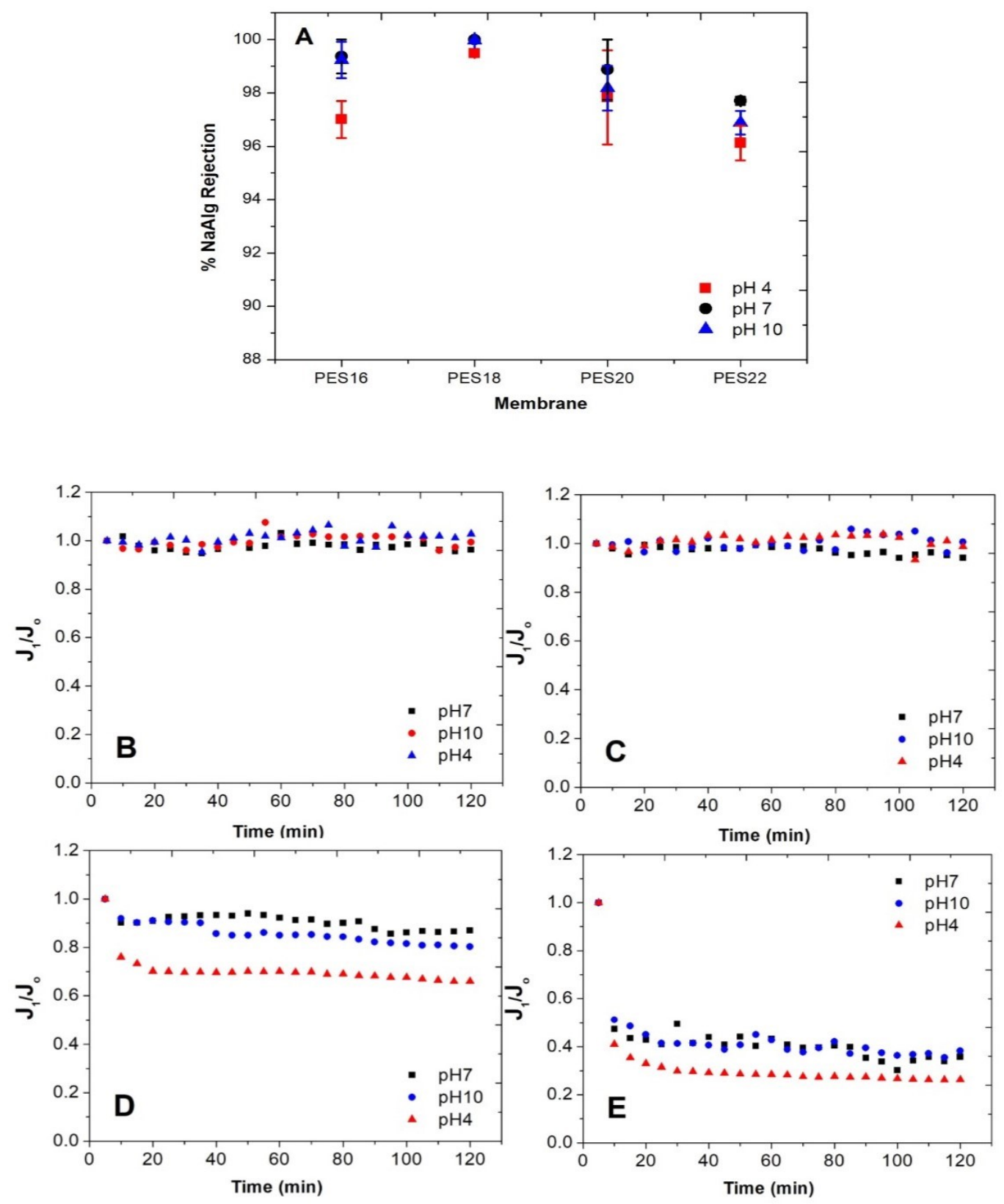

Figure 4.12: Influence of solution $\mathrm{pH}$ on $\mathrm{NaAlg}$ rejection coefficients (A), and fouling behaviour of (B) PES22, (C) PES20, (D) PES18, and (E) PES16 membrane.

To further assess the scope of polysaccharide retention and adsorptive fouling on UF membranes, three concentrations of NaAlg were used as a feed. As shown in Figure 4.13, all membranes had a very small rise in their retention coefficients and relative flux reduction with increasing initial feed concentration. This suggested that alginate adsorption, inside the membrane pores and on the surface, has readily taken place, even at the low alginate concentration (20 ppm), especially on the $100 \mathrm{kDa}$ membrane, and to a lower extent on the $35 \mathrm{kDa}$ membrane. Whereas, at $100 \mathrm{ppm}$ concentration, some 
Chapter Four - Investigation of Polyethersulfone polymer concentration on UF membrane characteristics and performance; a comprehensive studv

flux reduction for the PES20 was observed but not for the PES22 membrane. Disagreement can be seen between these results and previous research reported by Susanto et al., who found that a $10 \mathrm{kDa}$ UF membrane had fouling behaviour that was very influenced by the NaAlg concentration (Susanto et al., 2008). This difference is likely associated with the difference in membrane properties, experimental conditions and feed characteristics used in the measurements.
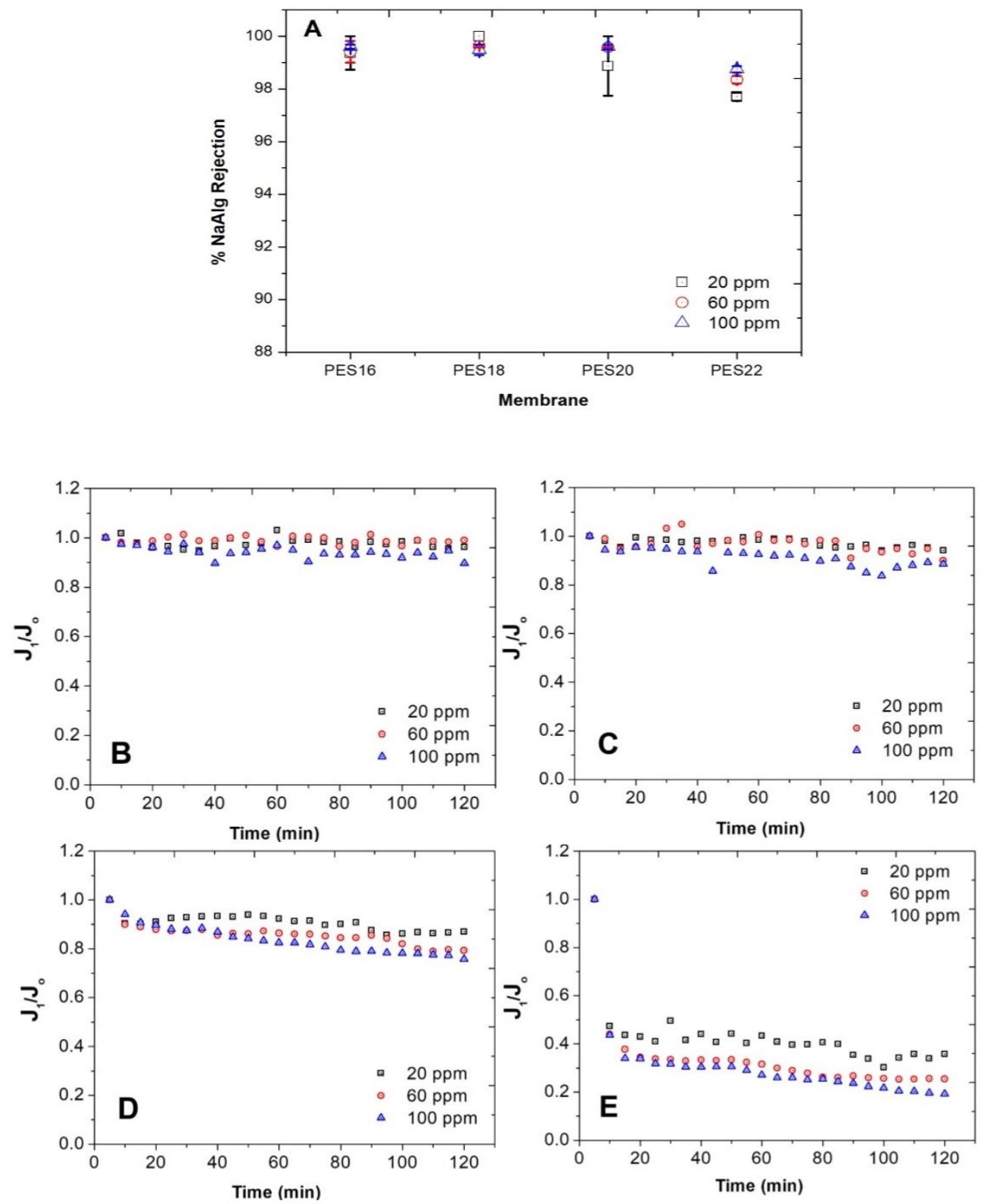

Figure 4.13: Influence of solution initial feed concentration on NaAlg rejection coefficients (A), and fouling behaviour of (B) PES22, (C) PES20, (D) PES18, and (E) PES16 membrane. 
Chapter Four - Investigation of Polyethersulfone polymer concentration on UF membrane characteristics and performance; a comprehensive study

The protein retention and fouling behaviour were also investigated in the filtration of the UF membranes using BSA. Similar to the retention trend observed with other model organics, BSA retention was found to be influenced by the feed solution chemistry $(\mathrm{pH})$, where lower rejection values were observed at acidic conditions for all membranes, Figure 4.14A. It should be noted that retention and permeate flux behaviour in UF membranes can be related to different mechanisms, for instance, different experimental conditions may result in different fouling mechanisms or protein characteristics (Huisman et al., 2000). Depending on the pore size distribution of the membrane applied for BSA filtration, uneven contribution for protein fouling mechanisms was observed on the membranes. As previously observed with HA and NaAlg filtration, PES20 and PES22 did not exhibit apparent relative flux declines due to the insufficient concentration and filtration time required to foul the membranes with BSA. In addition, the narrow pore distribution of these membranes prevented pore blocking, Figure 4.14B, and Figure 4.14C. In fact, the membrane fouling process with protein is complex due to the multiple interactions between membrane surfaces and protein molecules, and intramolecular interactions of the protein. The fouling can be interpreted as a two-stage process (Mo et al., 2008). According to Huisman et al. (2000), BSA-membrane hydrophobic interactions are dominant at the initial stage of filtration, while the high fouling regime that dictates the overall performance, was attributed to protein-protein interactions. For PES18 membrane, the relative flux pattern is more pronounced, particularly under acidic conditions. The initial hydrophobic interactions between the protein and membrane were marginal and did not cause any tangible fouling before the first $40 \mathrm{~min}$ of filtration. While slight flux decline commenced after $40 \mathrm{~min}$ of filtration demonstrating the dominant role of solute-solute electrostatic interactions on the performance of the membrane, Figure 4.14D. This can be explained as follow; at $\mathrm{pH}$ below the IEP point of BSA, the protein molecules possess positive charges while the membrane continued to have a negative zeta potential over the entire $\mathrm{pH}$ range, as indicated in section 4.3.4. At this point, the electrostatic repulsion between BSA and the membrane surface was significantly reduced, and the membrane surface acquired the BSA electrostatic charge as a result of adsorption, leading to a faster deposition rate on the surface of the fouled membrane. For the large MWCO membranes (PES16), the relative flux during BSA filtration manifested only small decline during the very first minutes of the filtration experiment, 
Chapter Four - Investigation of Polyethersulfone polymer concentration on UF membrane characteristics and performance; a comprehensive study

at neutral or alkaline feed solution condition, Figure 4.14E. This was significantly different to what was observed during the HA and NaAlg filtration utilising the same membrane, this reduction in relative permeate flux progressed gradually without reaching any steady-state after 120 min of filtration. Arguably, a simultaneous BSA adsorption could have occurred within the pores and at the membrane's surface, during the initial 10 min stage, due to the wide pore size range of the membrane. Next, as the filtration proceeds, accumulative adsorption of BSA-BSA fouled membrane will continue to build up a thicker layer of protein on the initial monolayer. The flux decline behaviour was even more severe in the acidic condition, which was facilitated by the weak protein-membrane interactions at low pH (Li et al., 2007b). Another possible explanation for the lower relative flux, in this case, is that high level of protein denaturation that was induced at acidic conditions, which would consequently form aggregations at $\mathrm{pH}$ below the IEP (Pincet et al., 1994). 
Chapter Four - Investigation of Polyethersulfone polymer concentration on UF membrane characteristics and performance; a comprehensive study
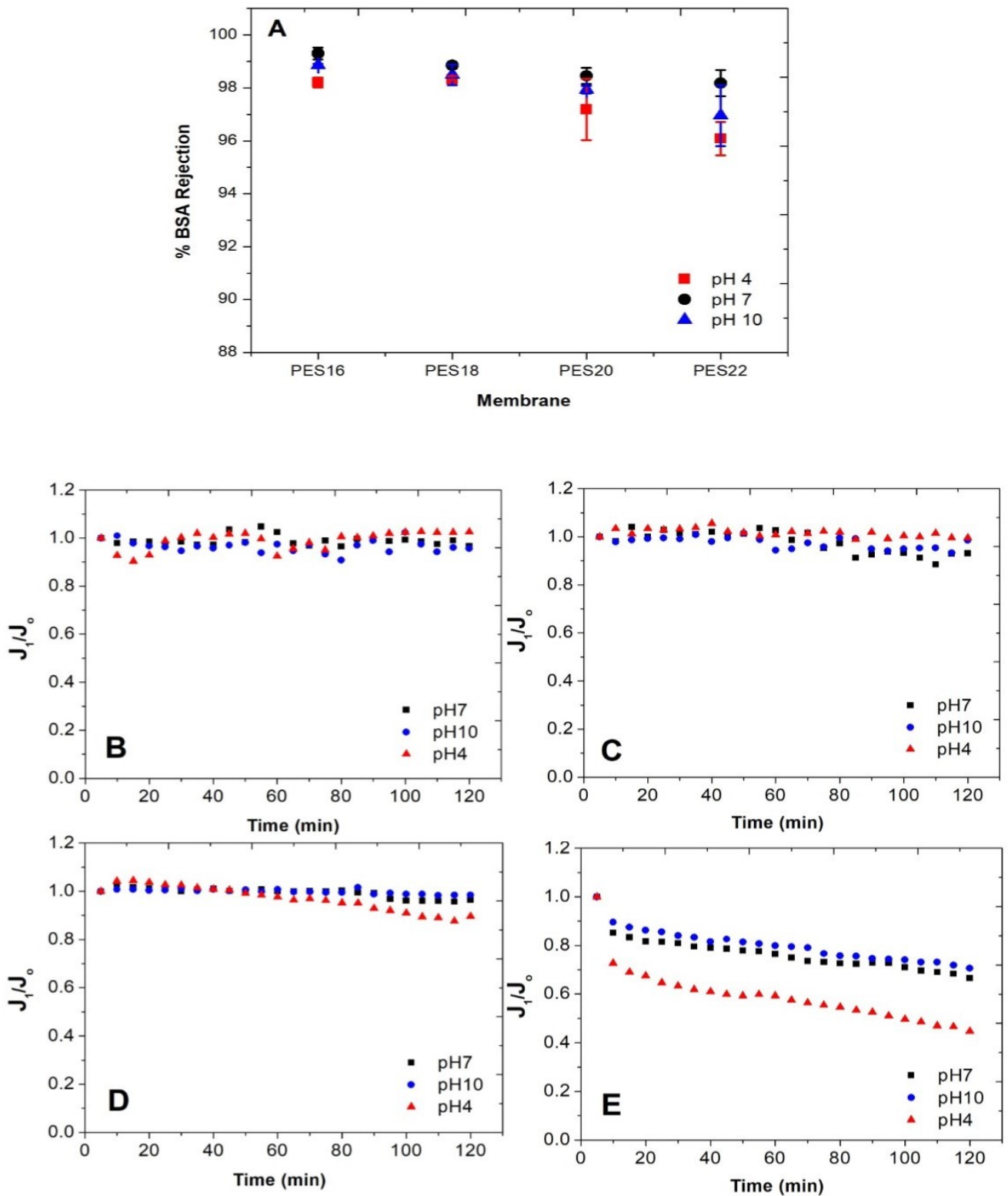

Figure 4.14: Influence of solution $\mathrm{pH}$ on BSA rejection coefficients (A), and fouling behaviour of (B) PES22, (C) PES20, (D) PES18, and (E) PES16 membrane.

In regard to the influence of BSA concentration on the retention of the membranes, Figure 4.15A indicated that all membranes maintained high rejection characteristics ( 98 - $99.5 \%$ ) to BSA, with a very slight improvement in retention coefficients of membranes as the initial feed concentration increased. While the relative flux decline was only significant for membranes with wide porous structure (PES18 and PES16), Figure 4.15B-Figure 4.15E. The reason behind this flux reduction was because of a higher mass transfer coefficient at the higher concentration and a thicker cake layer would appear earlier on the surface of membranes. 
Chapter Four - Investigation of Polyethersulfone polymer concentration on UF membrane characteristics and performance; a comprehensive studv
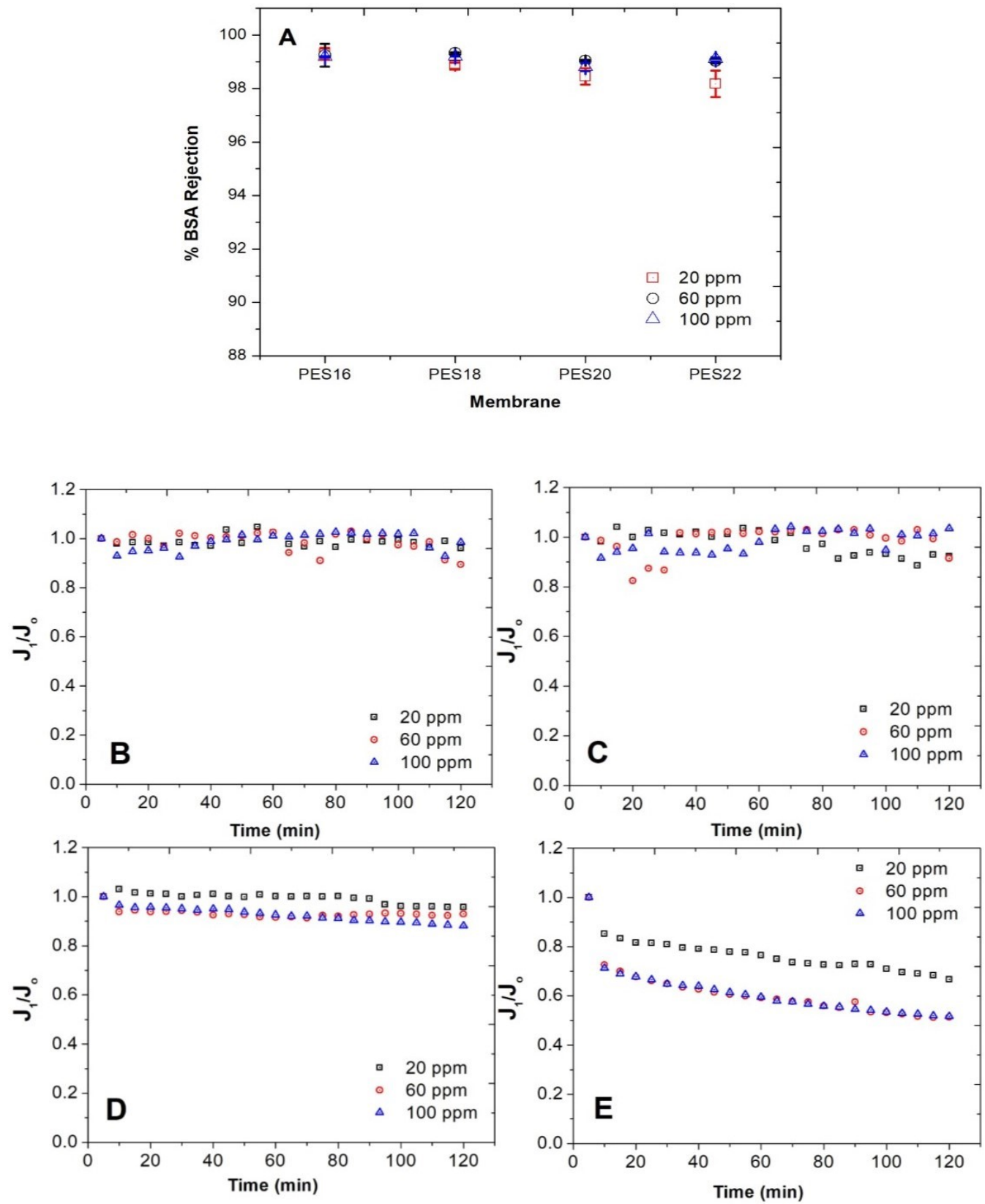

Figure 4.15: Influence of solution initial feed concentration on BSA rejection coefficients (A), and fouling behaviour of (B) PES22, (C) PES20, (D) PES18, and (E) PES16 membrane.

PWF measurements after back-flushing indicated that despite the NaAlg manifested higher relative flux decline, it was mostly recoverable. This was opposite to that observed with HA and BSA, which showed significant irreversible flux, mainly for wide cut-off membranes. Data of total, reversible and irreversible fouling in membranes as a function of $\mathrm{pH}$ and concentration are presented in Table 4.5 and Table 4.6. As shown in the tables, the tide PES22 and PES20 exhibited negative fouling parameters 
Chapter Four - Investigation of Polyethersulfone polymer concentration on UF membrane characteristics and performance; a comprehensive study

with HA filtration under neutral and alkaline conditions, while they were marginally fouled with other organic foulants. The effect of solution chemistry and initial concentration commenced being more obvious with protein and polysaccharide filtration using the $35 \mathrm{kDa}$ (PES18 membrane). The effects of the latter forms of fouling are mostly reversed by backwashing. The most severe fouling was associated with the PES16 membrane at acidic conditions, as illustrated earlier. About 63, 73 and $55 \%$ reduction in the original flux of the PES16 membrane was observed for HA, NaAlg, and BSA, respectively. Almost $64 \%$ of this flux decline was recovered when using the alginate while only 9 and $12 \%$ could be restored when using HA and BSA for the same conditions.

Table 4.5: Total, reversible and irreversible fouling data of membranes, as a function of initial feed solution $\mathrm{pH}$

\begin{tabular}{|c|c|c|c|c|c|c|c|c|c|c|c|c|}
\hline \multirow[t]{2}{*}{ HA } & \multicolumn{3}{|c|}{ PES22 } & \multicolumn{3}{|c|}{ PES20 } & \multicolumn{3}{|c|}{ PES18 } & \multicolumn{3}{|c|}{ PES16 } \\
\hline & pH4 & $\mathrm{pH} 7$ & $\mathrm{pH} 10$ & $\mathrm{pH} 4$ & $\mathrm{pH} 7$ & pH10 & $\mathrm{pH} 4$ & $\mathrm{pH} 7$ & pH10 & $\overline{\mathrm{pH} 4}$ & $\mathrm{pH} 7$ & $\mathrm{pH} 10$ \\
\hline $\mathrm{F}_{\mathrm{T}}$ & 5.993 & -9.342 & -8.094 & 4.854 & -7.71 & 3.373 & 15.61 & 1.002 & -0.97 & 63.41 & 53.83 & 57.719 \\
\hline $\mathrm{F}_{\mathrm{Rev}}$ & 8.508 & 0.105 & -2.253 & 4.358 & -5.83 & 2.682 & 4.133 & 0.733 & 1.245 & 9.2841 & 5.464 & 8.7039 \\
\hline $\mathrm{F}_{\text {IIr }}$ & -2.52 & -9.447 & -5.841 & 0.496 & -1.88 & 0.691 & 11.48 & 0.269 & -2.22 & 54.126 & 48.37 & 49.015 \\
\hline \multirow[t]{2}{*}{ NaAlg } & \multicolumn{3}{|c|}{ PES22 } & \multicolumn{3}{|c|}{ PES20 } & \multicolumn{3}{|c|}{ PES18 } & \multicolumn{3}{|c|}{ PES16 } \\
\hline & pH4 & $\mathrm{pH} 7$ & $\mathrm{pH} 10$ & pH4 & $\mathrm{pH} 7$ & $\mathrm{pH} 10$ & $\mathrm{pH} 4$ & $\mathrm{pH} 7$ & pH10 & $\mathrm{pH} 4$ & $\mathrm{pH} 7$ & $\mathrm{pH} 10$ \\
\hline $\mathrm{F}_{\mathrm{T}}$ & 4.188 & 1.521 & 4.22 & 3.886 & 4.414 & 2.659 & 34.89 & 13.17 & 20.24 & 73.471 & 62.41 & 60.641 \\
\hline $\mathrm{F}_{\mathrm{Rev}}$ & 1.742 & 0.181 & 1.807 & 3.676 & 3.729 & 2.071 & 30.84 & 6.955 & 17.52 & 63.851 & 51.1 & 52.316 \\
\hline $\mathrm{F}_{\text {IIr }}$ & 2.446 & 1.34 & 2.413 & 0.21 & 0.685 & 0.588 & 4.049 & 6.212 & 2.727 & 9.62 & 11.31 & 8.3257 \\
\hline \multirow[t]{2}{*}{ BSA } & \multicolumn{3}{|c|}{ PES22 } & \multicolumn{3}{|c|}{ PES20 } & \multicolumn{3}{|c|}{ PES18 } & \multicolumn{3}{|c|}{ PES16 } \\
\hline & $\mathrm{pH} 4$ & $\mathrm{pH} 7$ & $\mathrm{pH} 10$ & $\mathrm{pH} 4$ & $\mathrm{pH} 7$ & pH10 & $\mathrm{pH} 4$ & pH7 & pH10 & $\overline{\mathrm{pH} 4}$ & $\mathrm{pH} 7$ & $\mathrm{pH} 10$ \\
\hline $\mathrm{F}_{\mathrm{T}}$ & 3.636 & 4.211 & 4.401 & 3.631 & 2.222 & 2.453 & 11.96 & 4.67 & 2.095 & 55.656 & 33.61 & 29.428 \\
\hline $\mathrm{F}_{\mathrm{Rev}}$ & 1.571 & 2.456 & 1.738 & 2.46 & 0.461 & 0.231 & 1.145 & 1.141 & 0.257 & 12.158 & 8.745 & 5.779 \\
\hline $\mathrm{F}_{\text {IIT }}$ & 2.065 & 1.755 & 2.663 & 1.171 & 1.76 & 2.222 & 10.81 & 3.529 & 1.838 & 43.497 & 24.87 & 23.649 \\
\hline
\end{tabular}


Chapter Four - Investigation of Polyethersulfone polymer concentration on UF membrane characteristics and performance; a comprehensive study

Table 4.6: Total, reversible and irreversible fouling data of membranes, as a function of initial feed concentration.

\begin{tabular}{|c|c|c|c|c|c|c|c|c|c|c|c|c|}
\hline \multirow[t]{2}{*}{ HA } & \multicolumn{3}{|c|}{ PES22 } & \multicolumn{3}{|c|}{ PES20 } & \multicolumn{3}{|c|}{ PES18 } & \multicolumn{3}{|c|}{ PES16 } \\
\hline & $20 \mathrm{ppm}$ & $60 \mathrm{ppm}$ & $100 \mathrm{ppm}$ & $20 \mathrm{ppm}$ & $60 \mathrm{ppm}$ & $100 \mathrm{ppm}$ & $20 \mathrm{ppm}$ & $60 \mathrm{ppm}$ & $100 \mathrm{ppm}$ & $20 \mathrm{ppm}$ & $60 \mathrm{ppm}$ & $100 \mathrm{ppm}$ \\
\hline $\mathrm{F}_{\mathrm{T}}$ & -9.34 & -9.945 & -10.51 & -7.708 & -3.69 & -8.45 & 1.002 & 1.87 & 2.086 & 53.829 & 55.73 & 60.781 \\
\hline $\mathrm{F}_{\mathrm{Rev}}$ & 0.105 & -5.701 & -5.226 & -5.83 & -2.96 & -7.51 & 0.733 & 0.144 & 0.454 & 5.4636 & 6.72 & 13.636 \\
\hline $\mathrm{F}_{\text {IIr }}$ & -9.45 & -4.243 & -5.288 & -1.878 & -0.74 & -0.94 & 0.269 & 1.726 & 1.632 & 48.366 & 49.01 & 47.144 \\
\hline \multirow[t]{2}{*}{$\mathrm{NaAlg}$} & \multicolumn{3}{|c|}{ PES22 } & \multicolumn{3}{|c|}{ PES20 } & \multicolumn{3}{|c|}{ PES18 } & \multicolumn{3}{|c|}{ PES16 } \\
\hline & $20 \mathrm{ppm}$ & $60 \mathrm{ppm}$ & $100 \mathrm{ppm}$ & $20 \mathrm{ppm}$ & $60 \mathrm{ppm}$ & $100 \mathrm{ppm}$ & $20 \mathrm{ppm}$ & $60 \mathrm{ppm}$ & $100 \mathrm{ppm}$ & $20 \mathrm{ppm}$ & $60 \mathrm{ppm}$ & $100 \mathrm{ppm}$ \\
\hline $\mathrm{F}_{\mathrm{T}}$ & 1.521 & 2.994 & 9.408 & 4.414 & 4.743 & 10 & 13.17 & 20.61 & 24.85 & 62.408 & 75.02 & 78.529 \\
\hline $\mathrm{F}_{\mathrm{Rev}}$ & 0.181 & 2.283 & 3.188 & 3.729 & 4.117 & 7.2 & 6.955 & 19.05 & 23.41 & 51.097 & 66.99 & 70.41 \\
\hline $\mathrm{F}_{\text {IIr }}$ & 1.34 & 0.71 & 6.22 & 0.685 & 0.626 & 2.8 & 6.212 & 1.562 & 1.442 & 11.311 & 8.022 & 8.1197 \\
\hline \multirow[t]{2}{*}{ BSA } & \multicolumn{3}{|c|}{ PES22 } & \multicolumn{3}{|c|}{ PES20 } & \multicolumn{3}{|c|}{ PES18 } & \multicolumn{3}{|c|}{ PES16 } \\
\hline & $20 \mathrm{ppm}$ & $60 \mathrm{ppm}$ & $100 \mathrm{ppm}$ & $20 \mathrm{ppm}$ & $60 \mathrm{ppm}$ & $100 \mathrm{ppm}$ & $20 \mathrm{ppm}$ & $60 \mathrm{ppm}$ & $100 \mathrm{ppm}$ & $20 \mathrm{ppm}$ & $60 \mathrm{ppm}$ & $100 \mathrm{ppm}$ \\
\hline $\mathrm{F}_{\mathrm{T}}$ & 4.211 & 13.53 & 10.4 & 4.571 & 3.861 & 2.903 & 4.67 & 9.576 & 12.06 & 33.613 & 49.06 & 48.578 \\
\hline $\mathrm{F}_{\mathrm{Rev}}$ & 2.456 & 10.54 & 8.14 & 2.811 & 2.683 & 2.107 & 1.141 & 4.314 & 2.526 & 8.7454 & 8.357 & 17.23 \\
\hline $\mathrm{F}_{\text {Irr }}$ & 1.755 & 2.988 & 2.26 & 1.76 & 1.179 & 0.796 & 3.529 & 5.262 & 9.533 & 24.867 & 40.7 & 31.348 \\
\hline
\end{tabular}

\subsection{Conclusions}

The influence of PES polymer concentration on the morphologies and surface characteristics of asymmetric UF membranes was investigated at identical fabrication conditions. The variation in the casting solution viscosity has led up to a significant distinction in the pore size and pore size distribution, pure water flux, mechanical properties and cross-section morphologies, while this impact was trivial on water contact angle, surface roughness parameters and zeta potential of the membrane. As the casting solution concentration decreases from 22-16 wt.\%, the pore size increases from 2.1-10.7 $\mathrm{nm}$ along with wider pore size distribution curves and lower density. The molecular weight cut-off of UF membranes produced in this work is corresponding to the range of commercially available UF membranes $(6,10,35$ and 100) $\mathrm{kDa}$ for PES22, PES20, PES18, and PES22, respectively. The permeability was also dramatically increased from $3 \pm 0.2 \mathrm{l} / \mathrm{m}^{2}$.hr.bar (PES22) into $140 \pm 7.5 \mathrm{l} / \mathrm{m}^{2} . \mathrm{hr}$.bar (PES16). This permeability enhancement was attributed to the more open structure observed by SEM for the membranes with lower casting solution viscosity. That was indeed resulted in a lower mechanical strength, elongation and Young modulus by almost half of their 
Chapter Four - Investigation of Polyethersulfone polymer concentration on UF membrane characteristics and performance; a comprehensive study

values at $22 \mathrm{wt} . \%$ (PES22). However, the influence of solution viscosity on membrane surface charge has showcased a little increase in the negativity of membranes as their concentration decrease. Similarly, a slight increase in the values of $R_{a}, R_{m s}$, and $R_{\max }$. As well as an improvement in the hydrophilicity was observed as the PES concentration decreases. Despite membranes are made from the same polymer and under same conditions, this little negativity and low contact angle could result from the higher surface area correlated to the higher roughness parameters at low PES concentration. Table 4.7 below summarizes the role of PES concentration on the characteristics of membranes.

Table 4.7: Summary for PES wt $\%$ influence on characteristics of membranes.

\begin{tabular}{lllll}
\hline Characteristics & PES16 & PES18 & PES20 & PES22 \\
\hline AFM $\left(\mathrm{R}_{\mathrm{a}}\right)$ & 4.54 & 3.79 & 3.26 & 1.81 \\
\hline Mean pore size $(\mathrm{nm})$ & 10.79 & 4.4 & 2.9 & 2.17 \\
\hline Zeta potential & -7.78 & -6.5 & -6.8 & -5.4 \\
\hline Mechanical strength $(\mathrm{MPa})$ & 3.37 & 4.39 & 5.39 & 6.35 \\
\hline PWF $\left(1 / \mathrm{m}^{2} . \mathrm{hr}\right)$ & 560 & 172 & 48 & 12 \\
\hline Contact angle & 64 & 66.5 & 67.7 & 68.7 \\
\hline
\end{tabular}

To better understand the role of membranes surface properties, a comparison was made to provide valuable insight into details of organic molecules-membrane interactions when challenged with the natural organic matter, polysaccharides and proteins. The apparent variation in the rejection values of all membranes cut-off was not significant against each foulant, as the different MWCO characteristics obtained are compromised by different fouling mechanisms. While retention coefficients were in the following order; NaAlg $\geq$ BSA $>$ HA. Besides that, the relative flux decline was negligible for the low MWCO membranes, PES22 and PES20, within the short filtration duration of $120 \mathrm{~min}$ used in this research. In contrast, extremely low relative flux patterns were observed in the filtration with the $100 \mathrm{kDa}$ membrane (PES16). This was ascribed to the various fouling mechanisms contributed the total fouling of each membrane cut-off. Finally, although NaAlg manifested higher relative flux decline, it 
Chapter Four - Investigation of Polyethersulfone polymer concentration on UF membrane characteristics and performance; a comprehensive study

was mostly recoverable compared to HA and BSA filtration, which showed significant irreversible flux, mainly for the $100 \mathrm{kDa}$ membranes. 
Chapter Five - Fabrication and characterization of versatile metal/metal oxide carbon based hybrid nanostructures.

\section{Chapter Five}

\section{Fabrication and characterisation of versatile metal/metal oxide - carbon based hybrid nanostructures.}

\subsection{Introduction}

The decoration of CNTs with inorganic metal/metal oxide has recently shown as promising building blocks for many applications, e.g. fuel cells, gas sensors, solar cells, transparent conductive electrodes and hydrogen storage. CNTs have turned into an established material in considerable commercial products since its discovery in 1991 (Balasubramanian \& Burghard, 2005). Plentiful research endeavour has devoted to CNTs due to their tuneable thermal and electrical characteristics, high surface area to volume ratio, lightweight, novel superlative strength, and optical properties. On the other hand, the transition metal oxides are a remarkable family of inorganic NMs with plenteous properties in electronics, catalysis, optics, and magnetics. Their properties can be further tuned by varying their structure, morphology, and composition (Rao et al., 2006). Consequently, composite nanostructures based on inorganic metals and carbon could endow not only the unique characteristics of the two individual nanomaterials but also some novel properties as a result of their synergy (Vairavapandian et al., 2008).

CNTs have been proven as favourable templates for robustly tethering metallic NPs on their surfaces aiming for better stabilisation and dispersion of NPs (Chen \& Lu, 2010; Sotiriou \& Pratsinis, 2010). It is worth mentioning that pristine nanotubes exhibit limited attractive interactions with metal/metal oxide, and thus unsurprisingly poor 
Chapter Five - Fabrication and characterization of versatile metal/metal oxide carbon based hybrid nanostructures.

coating quality. Aside from that, the presence of metal catalytic particles and amorphous carbon, as impurities during CNTs preparation, could add additional burden to the intended application ( $\mathrm{Hu} \&$ Guo, 2011). However, purification and functionalization of CNTs could be established to negate the hydrophobic nature of CNTs and broaden their promising scope. For this reason, different linking groups, e.g. amine $\left(-\mathrm{NH}_{2}\right)$, sulfonic acid $\left(-\mathrm{SO}_{3} \mathrm{H}\right)$, carboxylic acid $(-\mathrm{COOH})$, hydroxyl $(-\mathrm{OH})$, or amide $\left(-\mathrm{CONH}_{2}\right)$ could be introduced to CNTs surface to facilitate linking different metal clusters to the nanotubes surface. Where these functional groups on the surface of CNTs can act as nucleation sites for reduction and precipitation of metal nanoparticles from various metal salts solution where no longer reduction agents are required (Yuan et al., 2008). Earlier attempts used to prepare such HNS from metal salt precursors tended to use reducing agents (Lordi et al., 2001; Wang et al., 2006a), hydrogen (Xue et al., 2001), or electrochemically by applying current through metal salt precursors with CNTs serving as an electrode for reducing metal cations on the surface of nanotubes (Day et al., 2005; Quinn et al., 2005).

This chapter encloses two themes; first, two loading level (10 and $20 \mathrm{wt} . \%)$ of AgNPs to the MWCNTs were synthesised via wet chemistry technique, and the deposition of the silver on the surface of nanotubes was modulated with microwave treatment. This was carried to assess and compare the influence of varying Ag loading wt.\% to CNTs on the morphological and antibacterial characteristics of mixed matrix membranes, as will be discussed in Chapter 6. In the second theme, four different HNS comprises $\mathrm{M} / \mathrm{MO}\left(\mathrm{Ag}, \mathrm{Al}_{2} \mathrm{O}_{3}, \mathrm{Fe}_{2} \mathrm{O}_{3}\right.$, and $\left.\mathrm{TiO}_{2}\right)$-MWCNTs have been synthesised. This was carried via same wet chemistry technique, then samples were calcinated inside a high-temperature tube furnace. The HNS synthesised herein were employed via novel route for TFN membrane applications in desalination, as will be discussed later in Chapter 7.

\subsection{Nanoscale materials fabrications}

\subsubsection{Purification and carboxylation of MWCNTs}

In most of CNTs applications, especially preparing nanocomposite membranes, the poor dispersion of nanotubes represents the major obstacle that needs to be tackled. CNTs tend to form bundles due to the strong $\pi-\pi$ stacking interactions with the 
Chapter Five - Fabrication and characterization of versatile metal/metal oxide carbon based hybrid nanostructures.

neighbouring nanotubes that would limit their dispersion in various solvents. Therefore, breaking these bundles into individual nanotubes is critical for optimising any of their applications. Nevertheless, presented metal catalytic particles and amorphous carbon, as impurities during the CNTs preparation, could add additional burden to the intended application (Hu \& Guo, 2011). However, purification and functionalization of CNTs have been found as effective tools for coping the hydrophobic nature of CNTs and broadening its promising scope.

To eliminate amorphous carbon and trace metal catalysts, and to carboxylate, the

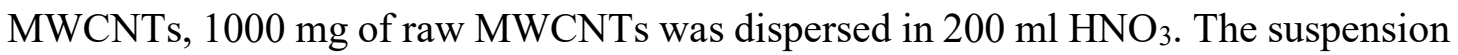
was treated for $10 \mathrm{~min}$ in a bath sonicator, then refluxed under $120^{\circ} \mathrm{C}$ for $48 \mathrm{~h}$, the reflux unit is shown in Figure 5.1. Later, the solution was cooled down at room temperature, then diluted with DI water. Finally, the resultant precursor was passed through a $0.22 \mu \mathrm{m}$ cellulose nitrate membrane, washed with plenty of DI water to neutralize the $\mathrm{pH}$ and dried in a vacuum oven (vacumcenter, Salvis LAB- Switzerland) at $40{ }^{\circ} \mathrm{C}$ overnight.

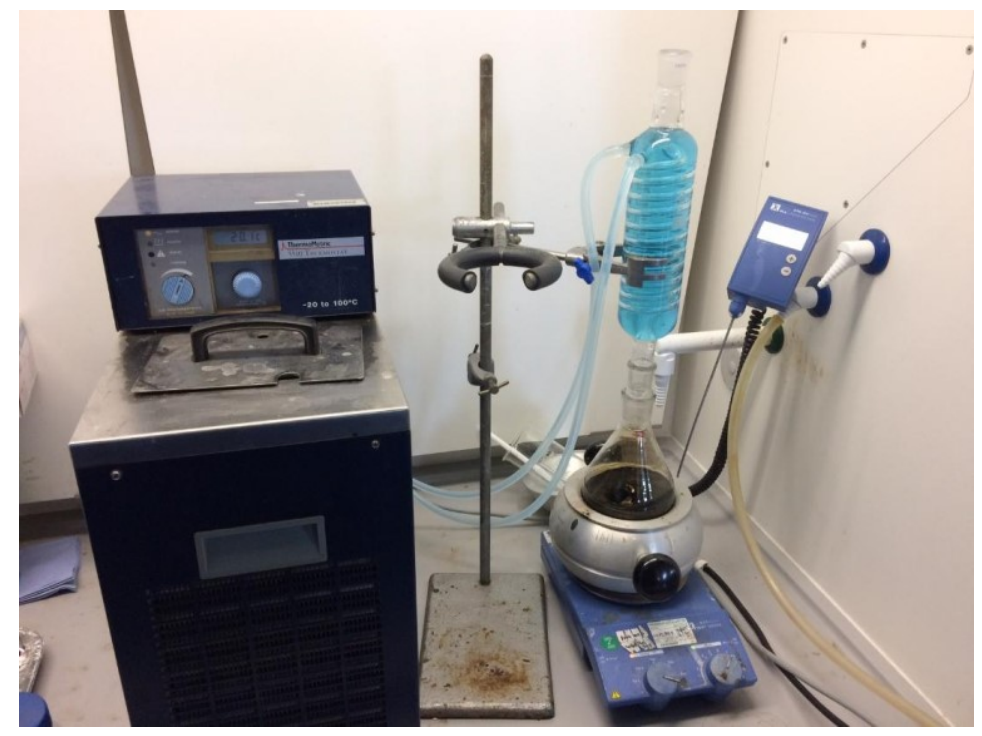

Figure 5.1: Reflux unit for functionalisation of nanotubes.

\subsubsection{Decoration of MWCNTs with M/MO nanoparticles}

A number of procedures are reported in the literature for decorating CNTs with metal nanoparticles. Basically, they have been divided into to two categories; Dry routes (Lin et al., 2009) and Wet chemistry (Chen \& Lu, 2010). The latter method, used in this work for the synthesis, initially involves chemical functionalization of CNTs to activate 
Chapter Five - Fabrication and characterization of versatile metal/metal oxide carbon based hybrid nanostructures.

the extremely inert graphite surface of CNTs in order to establish a uniform coverage of NP on the nanotubes, before the subsequent metal cations reduction (Hu \& Guo, 2011). Functionalization has been used to create linking groups (e.g., $-\mathrm{OH},-\mathrm{COOH}$ and $-\mathrm{C}=\mathrm{O}$ ) that ultimately facilitates linking different metal clusters to the nanotubes surface via covalent (Zamudio et al., 2006), noncovalent (Li et al., 2006) and electrostatic interactions (Kim \& Sigmund, 2004). The binding mechanism between CNTs and NPs could assist the design of these HNS, which influence their properties and applications indeed (Chen \& Lu, 2010).

In this thesis, for decorating the nanotubes with M/MO NPs, two groups of nanocomposite material (Table 5.1) were fabricated via an identical procedure except the final step when the physical deposition of the NPs on the surface of nanotubes is conducted. See schematic diagram (Figure 5.2) below, e.g. Ag-MWCNTs.

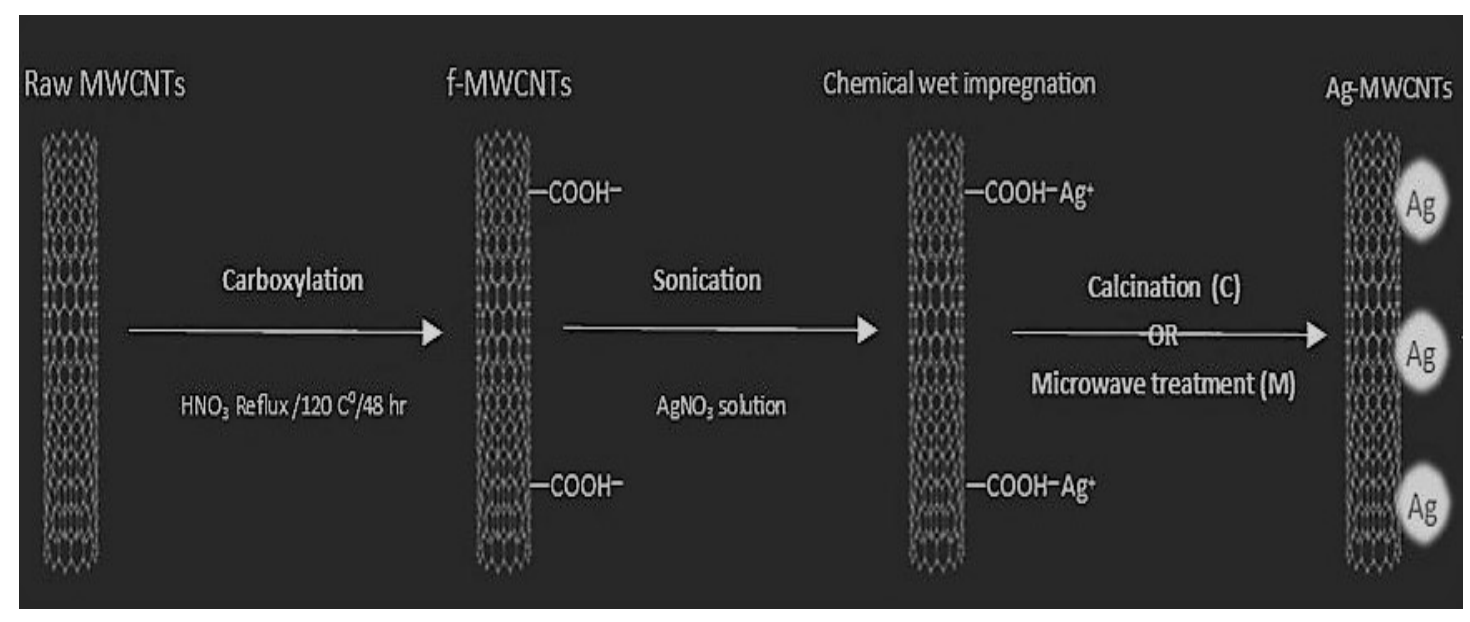

Figure 5.2: Schematic diagram for the HNS (Ag-MWCNTs) fabrication.

In the first group, 10 and 20 loading wt.\% of Ag to CNTs was used and the samples abbreviated as 10HNS and 20HNS respectively. A concentration of $1.17 \mathrm{mM}$ and 2.35 $\mathrm{mM}$ silver nitrate in $100 \mathrm{ml}$ methanol were bath sonicated individually for $60 \mathrm{~min}$ to yield 10 and 20 wt. \% silver on CNTs, respectively. Then, $127 \mathrm{mg}$ of carboxylated MWCNTs, prepared earlier, was added to the solution and sonicated for another $30 \mathrm{~min}$ utilising a probe sonicator (Figure 3.18 ) at $80 \%$ amplitude and 2 pulse on-off. An ice bath was utilised to maintain the precursors temperature at $20 \pm 2{ }^{\circ} \mathrm{C}$ during the sonication. Thereafter, the precursor was dried at $35-40 \mathrm{C}^{\circ}$ in the vacuum oven. Finally, to physically deposit the Ag NP on the CNTs surface, the sample was treated by 
Chapter Five - Fabrication and characterization of versatile metal/metal oxide carbon based hybrid nanostructures.

microwave irradiation for $10 \mathrm{~min}$. A domestic $1000 \mathrm{~W}$ microwave oven (Panasonic NN-CT579SBPQ) was used as the microwave source in all the experiments. In all microwave reactions, the sample was placed in a glass vial and microwaved at 1000 Watt power. Two Pyrex beakers $2 / 3$ full of water were placed in the microwave to prevent overheating and replaced with cold water every one minute.

For the second group, $75 \mathrm{mg} \mathrm{Fe}\left(\mathrm{NO}_{3}\right)_{3} .9 \mathrm{H}_{2} \mathrm{O}, 71.4 \mathrm{mg} \mathrm{Ti}\left(\mathrm{OCH}_{2} \mathrm{CH}_{2} \mathrm{CH}_{2} \mathrm{CH}_{3}\right)_{4}$, $138.99 \mathrm{mg} \mathrm{Al}\left(\mathrm{NO}_{3}\right)_{3} .9 \mathrm{H}_{2} \mathrm{O}$ and $40 \mathrm{mg} \mathrm{AgNO}_{3}$ was individually added into $100 \mathrm{ml}$ methanol and bath sonicated for $1 \mathrm{~h}$. Then, $100 \mathrm{mg}$ of carboxylated nanotubes were added and sonicated for another $30 \mathrm{~min}$ with a tip sonicator to yield about $10 \mathrm{wt} \%$ metal to the MWCNTs. Thereafter, the precursor was dried at $35-40{ }^{\circ} \mathrm{C}$ in a vacuum oven for 4 hrs. Finally, to convert metal salt into the metallic form on the surface of CNTs, each sample was calcinated in a high-temperature tube furnace (Figure 3.20) under inert conditions. Calcination was carried out by placing the sample in an aluminium pan inside the tube furnace under $450^{\circ} \mathrm{C}$ for $5 \mathrm{hr}$. Nitrogen or Argon, as an inert gas, was then passed through the glass tube at a flow rate $100 \mathrm{ml} / \mathrm{min}$ to avoid the thermal decomposition. Finally, the furnace was started after setting up the required time and temperature.

Table 5.1: Composition and decomposition technique used during the nanocomposite material fabrication.

\begin{tabular}{llll}
\hline Group one & & & \\
Sample ID & $\begin{array}{l}\text { M/MO- } \\
\text { MWCNTs ratio }\end{array}$ & Final treatment step & Application \\
$10 \mathrm{HNS}$ & $10 \mathrm{Ag}$ & Microwave & Antibacterial nanocomposite membranes \\
$20 \mathrm{HNS}$ & $20 \mathrm{Ag}$ & Microwave & Antibacterial nanocomposite membranes \\
\hline Group Two & & & \\
$\mathrm{Sample} \mathrm{ID}$ & $\begin{array}{l}\mathrm{M} / \mathrm{MO}- \\
\mathrm{MWCNTs} \mathrm{ratio}\end{array}$ & Final treatment step & Application \\
$\mathrm{Fe}_{2} \mathrm{O}_{3} \mathrm{CNTs}$ & $10 \mathrm{Fe}_{2} \mathrm{O}_{3}$ & Tube furnace & TFN for Desalination \\
$\mathrm{TiO}_{2} \mathrm{CNTs}$ & $10 \mathrm{TiO}_{2}$ & Tube furnace & TFN for Desalination \\
$\mathrm{Al}_{2} \mathrm{O}_{3} \mathrm{CNTs}$ & $10 \mathrm{Al}_{2} \mathrm{O}_{3}$ & Tube furnace & TFN for Desalination \\
$\mathrm{Ag} \mathrm{CNTs}$ & $10 \mathrm{Ag}$ & Tube furnace & TFN for Desalination \\
\hline
\end{tabular}


Chapter Five - Fabrication and characterization of versatile metal/metal oxide carbon based hybrid nanostructures.

\subsection{Characterisation of HNS.}

\subsubsection{FTIR spectra evaluation of MWCNTs}

Chemical treatment aiming for oxidation, substitution, addition, and hydrolysis of a material's surface is one of the familiar approaches to endow a specific functional group on that surface (Hilal et al., 2017). Applying concentrated $\mathrm{HNO}_{3}$ for p-MWCNT purification has enhanced both purity and partial oxidation of nanotubes where a number of functional groups, mainly carboxyl and hydroxyl, appeared after the acid treatment. Total Reflection Infrared spectra (FTIR) of p-MWNT and f-MWNTs from 700 to $4000 \mathrm{~cm}^{-1}$ is shown in (Figure 5.3). The broad well-defined peaks around 3392 $\mathrm{cm}^{-1}$ can be ascribed to the stretching mode of a hydroxyl $(-\mathrm{OH})$ bond (Somanathan et al., 2015). Notable peaks were observed around $2690 \mathrm{~cm}^{-1}$ that correspond to $\mathrm{C}-\mathrm{H}$ symmetric and asymmetric stretching vibration, which is responsible for the stability of nanotubes suspensions in the aqueous phase (Mkhondo \& Magadzu, 2014; Yudianti et al., 2011). IR regions around $1650 \mathrm{~cm}^{-1}$ are assigned to oscillation of carboxyl ($\mathrm{COOH})$ groups, while the broad weak peak observed at $1477 \mathrm{~cm}^{-1}$ is probably associated with $\mathrm{O}-\mathrm{H}$ bending deformation of $-\mathrm{COOH}$ acid groups (Moraes et al., 2011). The well-defined peak at $1064 \mathrm{~cm}^{-1}$ was correlated to the $\mathrm{C}-\mathrm{O}$ stretch vibration. Thus, FTIR has confirmed the presence of oxygen/acid functional groups induced after acid treatment.

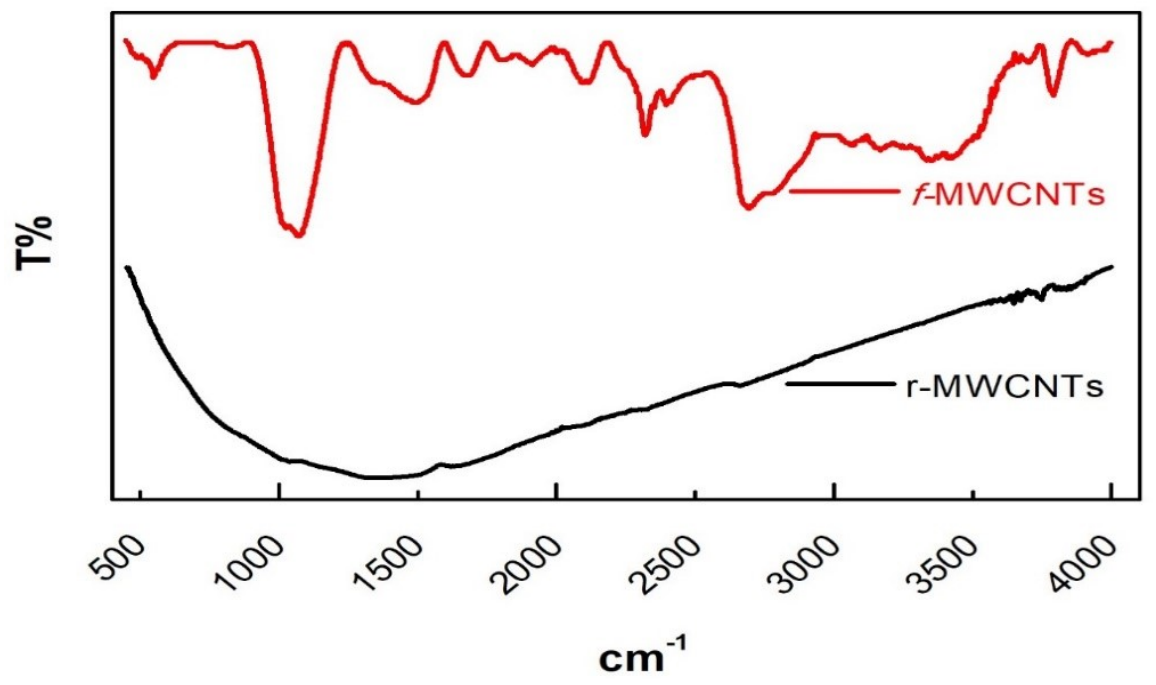

Figure 5.3: Fourier transform infrared spectroscopy (FT-IR) spectrum of p-MWCNTs and f-MWCNTs. 
Chapter Five - Fabrication and characterization of versatile metal/metal oxide carbon based hybrid nanostructures.

\subsubsection{Zeta potential measurements.}

Zeta potential measurement is the key parameter that takes control electrostatic interactions in particle dispersions and, as such, understanding the stability of colloidal dispersion is of great significance (Kaszuba et al., 2010). Measurements were carried out as a function of $\mathrm{pH}(3-11)$ to quantify the degree of functionalization for $\mathrm{f}$ MWCNTs, the role of metal/metal oxides deposition on the zeta potential of the HNS, and to evaluate the role of depositing different loading levels of Ag NPs on the surface of $f$-MWCNTs on their colloidal stability. It can be clearly seen that carboxylating the pristine nanotubes greatly influenced the zeta potential values (Figure 5.4).

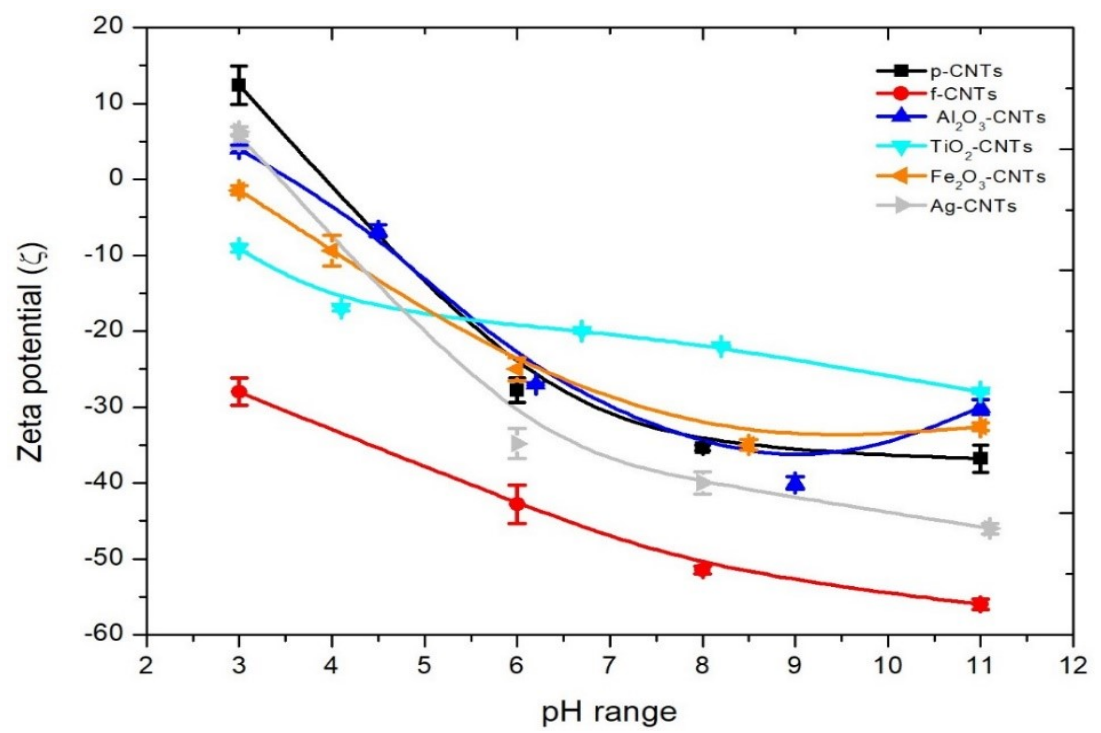

Figure 5.4: Zeta potential measurements as a function of $\mathrm{pH}$, for; raw, carboxylated nanotubes, and nanocomposites.

The isoelectric point of $\mathrm{p}$-MWCNTs located at $\mathrm{pH} 4$ has disappeared after functionalization where the plot shifted to more negative values even within a very high acidic environment. As confirmed in Section 5.3.1, the presence of novel functional groups has given rise to higher negatively charged nanotubes. Indeed, this enhancement in the value of zeta potential (e.g. -27 to -43 at $\mathrm{pH}$ 6) is expected to induce a better stability and dispersibility for nanotubes in the polymeric matrix (Ji, 2012), and also act as nucleation sites for the later deposition of M/MO NPs on the surface of nanotubes (Hu \& Guo, 2011). However, decorating the f-MWCNTs with metal/metal oxides nanoparticles has shifted the zeta potential values, for all nanocomposites over the entire $\mathrm{pH}$ range, to more positive values. This was ascribed to the role of positively 
Chapter Five - Fabrication and characterization of versatile metal/metal oxide carbon based hybrid nanostructures.

charged metal/metal oxides nanoparticles that contributed to the total charge of the nanocomposite structures (Alimohammadi et al., 2012).

Similarly, Ag NPs deposition on the nanotubes had also lowered zeta potential values for both $10 \mathrm{HNS}$ and $20 \mathrm{HNS}$ prepared with the aid of microwave treatment (Figure 5.5). This was ascribed to the positively charged Ag NPs contribution to the total charge of the nanostructures as indicated earlier. This contribution was higher in the case of $10 \mathrm{HNS}$ compared to that of $20 \mathrm{HNS}$ and was due to the higher surface to volume ratio of $\mathrm{Ag}$ in the $10 \mathrm{HNS}$ sample, as will be demonstrated in the next sections. Even though both hybrid nanostructures exhibited lower zeta values than f-MWCNTs, they are still higher than those of p-MWCNTs.

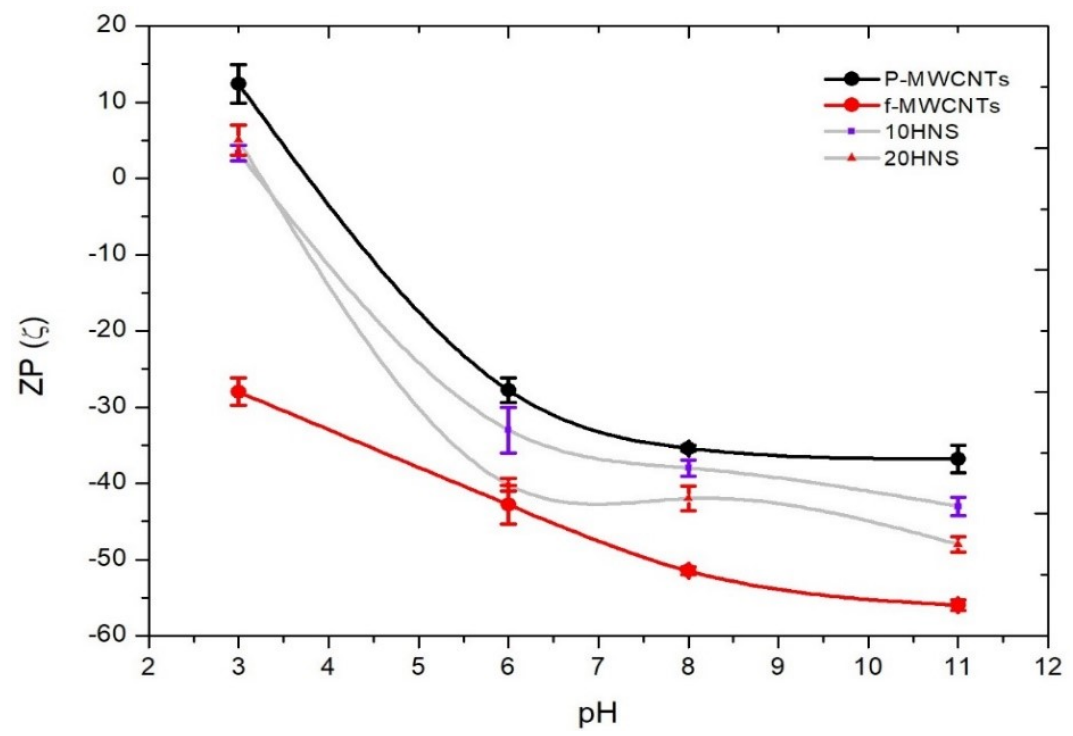

Figure 5.5: Zeta potential measurements as a function of $\mathrm{pH}$ for; raw, carboxylated and silver decorated nanotubes.

\subsubsection{X-ray diffraction analysis}

The XRD patterns of the crystalline structure of carbon nanotube samples and M/MOMWCNTs nanocomposites are shown in Figure 5.6. The carbon nanotubes, both pristine and functionalized (Figure 5.6A and Figure 5.6B), showed two diffraction peaks at $26^{\circ}$ and $43^{\circ}$ that correspond to the (002) and (100) reflections of the graphite layers' structure (COD 1200017 and COD 9011577) (Gunawan et al., 2011). These distinctive two diffraction peaks were also observed in all M/MO nanocomposites XRD patterns. It's clear that amorphous carbon component, observed in the p-MWCNTs sample (the broad medium peak $27^{\circ}-35^{\circ}$ ), has been removed after the acid treatment. After decorating the nanotubes with AgNPs, another three peaks were observed at $64^{\circ}$, 
Chapter Five - Fabrication and characterization of versatile metal/metal oxide carbon based hybrid nanostructures.

$44^{\circ}$ and $38^{\circ}$ that correspond to the (220), (200) and (111) reflections associated with $\mathrm{Ag}(0)$ as shown in Figure 5.6C. Since different phases of iron oxide (magnetite- $\mathrm{Fe}_{3} \mathrm{O}_{4}$, hematite- $\alpha-\mathrm{Fe}_{2} \mathrm{O}_{3}$, and maghemite- $\gamma-\mathrm{Fe}_{2} \mathrm{O}_{3}$ ) have very similar XRD patterns, it is hard for the XRD to distinguish between them (Liu et al., 2016). This scenario could be worse in the presence of another material to form a hybrid nanocomposite (such as carbon nanotubes in this work). However, the existence of diffraction peaks at $32.5^{\circ}$ (104), $50^{\circ}(024)$ and $63^{\circ}$ (300) could be deemed as a confirmation for $\alpha-\mathrm{Fe}_{2} \mathrm{O}_{3}$ formation, rather than other iron oxide phases, Figure 5.6D. For the other HNS, no visible XRD peaks attributed to $\mathrm{TiO}_{2}$ and $\mathrm{Al}_{2} \mathrm{O}_{3}$ were detected in the $\mathrm{TiO}_{2}-\mathrm{CNT}$ and $\mathrm{Al}_{2} \mathrm{O}_{3}-\mathrm{CNTs}$ spectra, Figure 5.6E and Figure 5.6F. This could be due to the lower extent of the crystalline metals, compared to the crystalline CNTs, used to prepare these HNS, leading to shielding of the metal peaks by those of the nanotubes, and/or the $\mathrm{TiO}_{2} \mathrm{XRD}$ peaks in the nanocomposite could be overlapped by the nanotubes peaks since the main peaks position of $\mathrm{TiO}_{2}$ at $25.4^{\circ}$ is close to the main diffraction peak of the nanotubes at $25.9^{\circ}$. The opposite of these findings was reported by $\mathrm{Yu}$ et al., as titanium masked the nanotubes peaks when $\mathrm{TiO}_{2}$ had a higher loading to that of nanotubes in the HNS ( $\mathrm{Yu}$ et al., 2005c). Further characterisation has been carried out later by Raman and XPS to avoid the XRD limitations and assert the HNS composition/oxidation state.

Similarly, decorating carbon nanotubes with silver nanoparticles (10HNS and 20HNS), under microwave treatment (Figure 5.6G and Figure 5.6H), showcased the three peaks that correspond to the (111), (200) and (220) reflections related with the Ag (0) (COD 9011607), as illustrated earlier in this section. Interestingly, the peak (002) assigned to CNTs characteristics displayed lower intensity than pristine and functionalized nanotubes after decoration with $\mathrm{Ag}$, where this reduction could be attributed to defects created on the surface of nanotubes during the decorating process (Yuan et al., 2008). Also, the peak was almost disappeared in the higher silver content sample (20HNS) due to the higher coverage rate of Ag NPs on the surface of nanotubes. Nevertheless, the XRD diffraction peaks of 10HNS are slightly widened due to the deposition of smaller metallic Ag-NPs existing as silver nanocrystal. 
Chapter Five - Fabrication and characterization of versatile metal/metal oxide carbon based hybrid nanostructures.
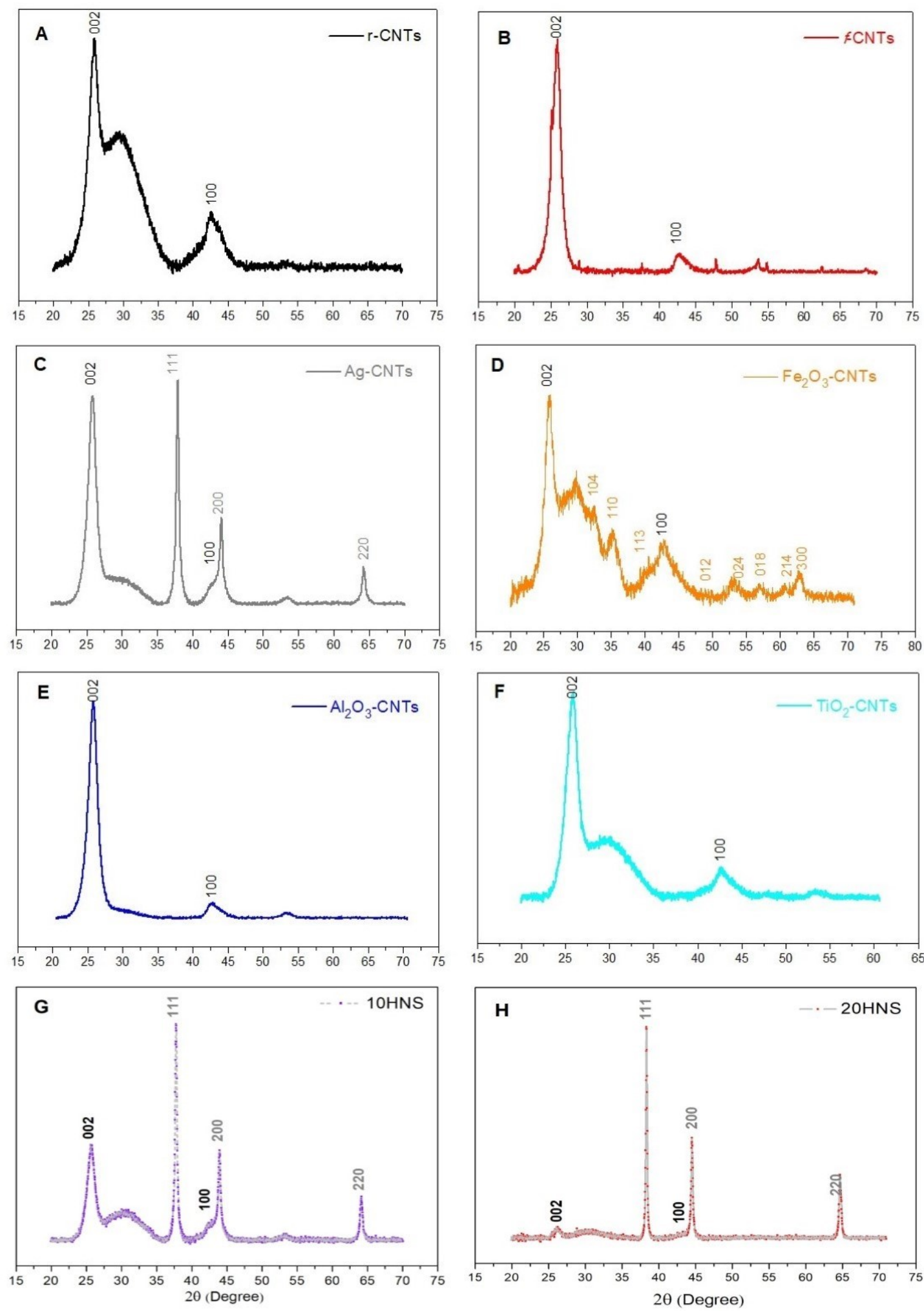

Figure 5.6: XRD patterns of (A) p-MWCNTs, (B) f-MWCNTs (C) Ag-CNTs, (D) $\mathrm{Fe}_{2} \mathrm{O}_{3}$-CNTs, (E) $\mathrm{Al}_{2} \mathrm{O}_{3}$-CNTs, (F) $\mathrm{TiO}_{2}$-CNTs, (G) 10HNS, and (H) 20HNS 
Chapter Five - Fabrication and characterization of versatile metal/metal oxide carbon based hybrid nanostructures.

\subsubsection{Microscopic observation and elemental composition}

Surface morphology of MWCNTs, prior and after M/MO decoration by both deposition routes, were observed by SEM. No apparent destruction was found in the structure of MWCNTs after treatment with concentrated $\mathrm{HNO}_{3}$. (Figure 5.7A and Figure 5.7B) show a clear cylindrical nanostructure as the bundles aligned together through the action of van der Waals forces. The only difference between those two samples was their purity and oxygen content as confirmed by elemental analysis (EDX). The residue of metallic catalysts was much less apparent in the f-MWCNTs sample than in the pristine one, indicating a higher degree of purity was achieved.
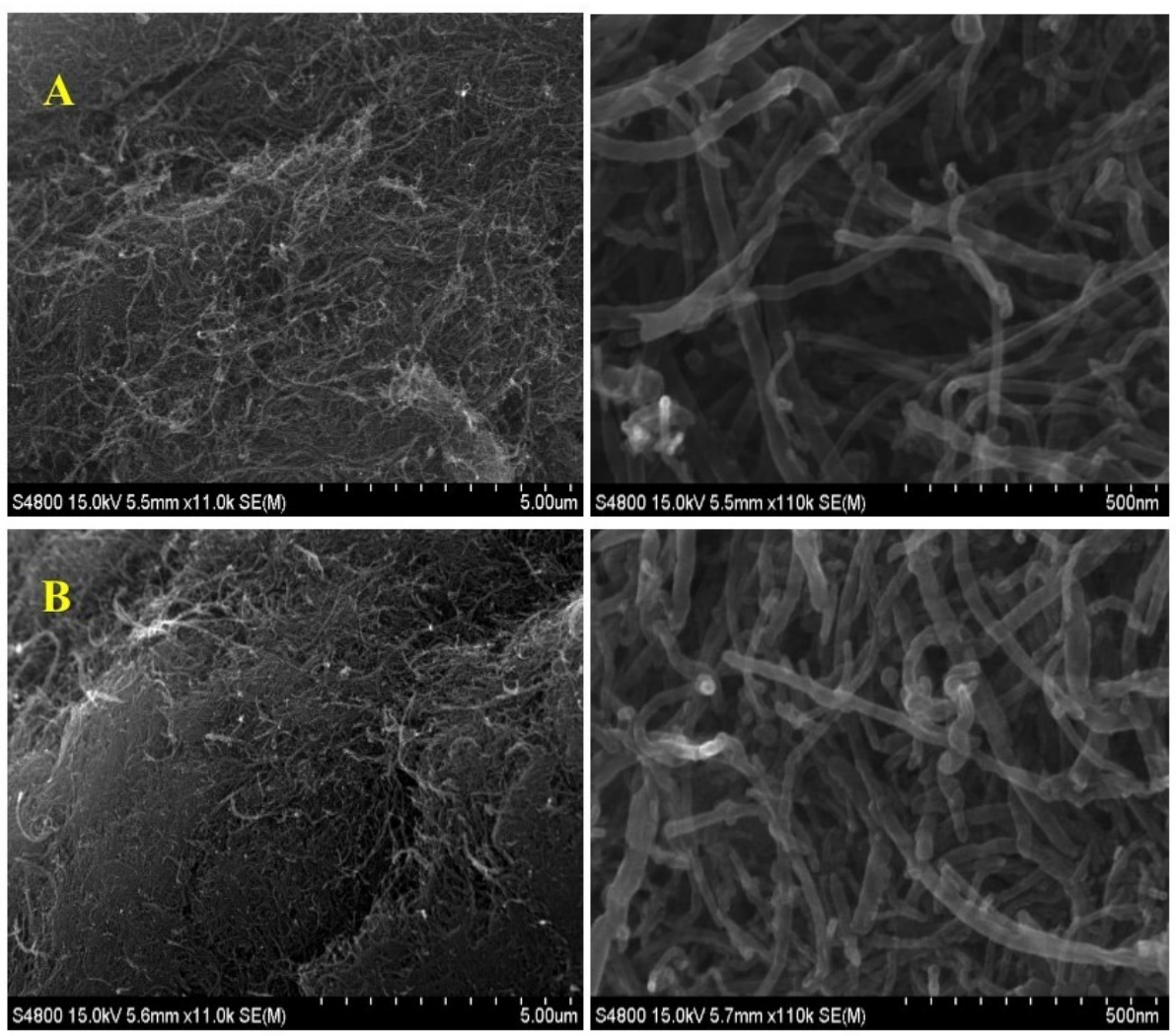

Figure 5.7: $5 \mu \mathrm{m}$ (Left) and $500 \mathrm{~nm}$ (Right) SEM images for (A) p-MWCNTs, (B) fMWCNTs.

Morphology of nanotubes, after decoration with metallic NPs, is presented in (Figure 5.8). No visible deposited metal/metal oxides NPs were observed on the surface of the nanocomposites by SEM imaging, despite the confirmed chemical composition results obtained from EDX (Figure 5.11C-Figure 5.11H) comparing to the r-CNTs and f-CNTs samples (Figure 5.11A and Figure 5.11B). Likewise, it was difficult to observe Ag NPs deposition in the 10HNS sample (Figure 5.9A), since an identical concentration 
Chapter Five - Fabrication and characterization of versatile metal/metal oxide carbon based hybrid nanostructures.

(10 wt.\%) to other M/MO NPs was used during the nanocomposite synthesis (Table 5.1). Probably, this was due to the small size of metal/metal oxides NPs packaged on the surface of MWCNTs. However, homogenous distribution, without distinctive aggregation, was observed in the 20HNS sample due to the higher loading wt.\% of Ag used in preparation (Figure 5.9B). This observation of larger Ag-NPs could be due to the limited number of active anchoring sites (e.g. defects) on the surface of CNTs. Indeed, the active sites were oversaturated with the higher Ag content in the 20HNS. Consequently, the remaining decomposed silver continued deposition onto the previously anchored seeds to form larger size AgNPs (Lin et al., 2011). Aside from that, the required irradiation period was critical to achieve a homogeneous deposition of $\mathrm{Ag}$ on the surface of nanotubes, as illustrated in Figure 5.10. 
Chapter Five - Fabrication and characterization of versatile metal/metal oxide carbon based hybrid nanostructures.
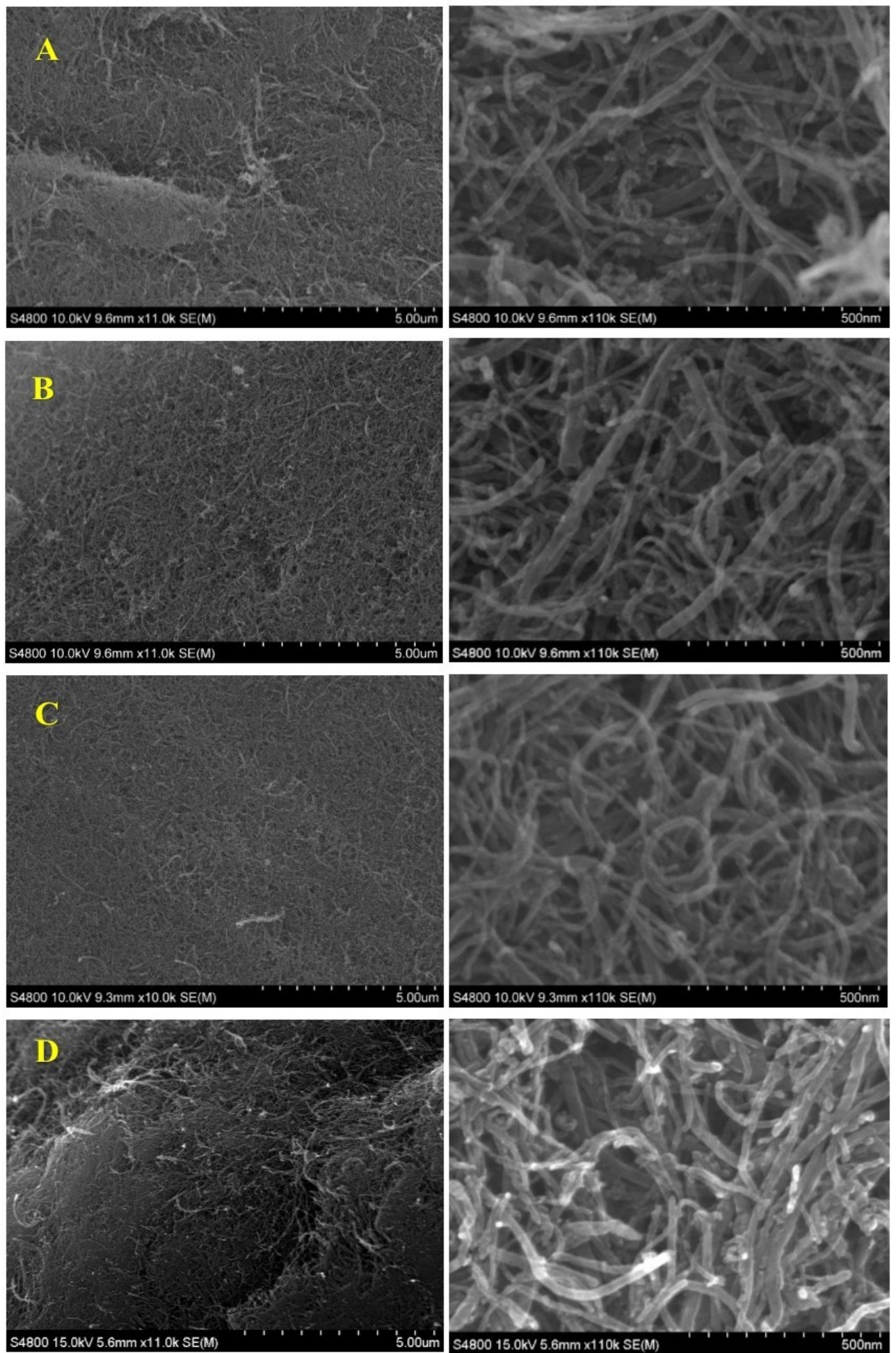

Figure 5.8: $\mu \mathrm{m}$ (Left) and $500 \mathrm{~nm}$ (Right) SEM images for (A) $\mathrm{Fe}_{2} \mathrm{O}_{3}-\mathrm{CNTs}$, (B) $\mathrm{TiO}_{2}$-CNTs, (C) $\mathrm{Al}_{2} \mathrm{O}_{3}-\mathrm{CNTs}$, and (D) Ag-CNTs. 
Chapter Five - Fabrication and characterization of versatile metal/metal oxide carbon based hybrid nanostructures.
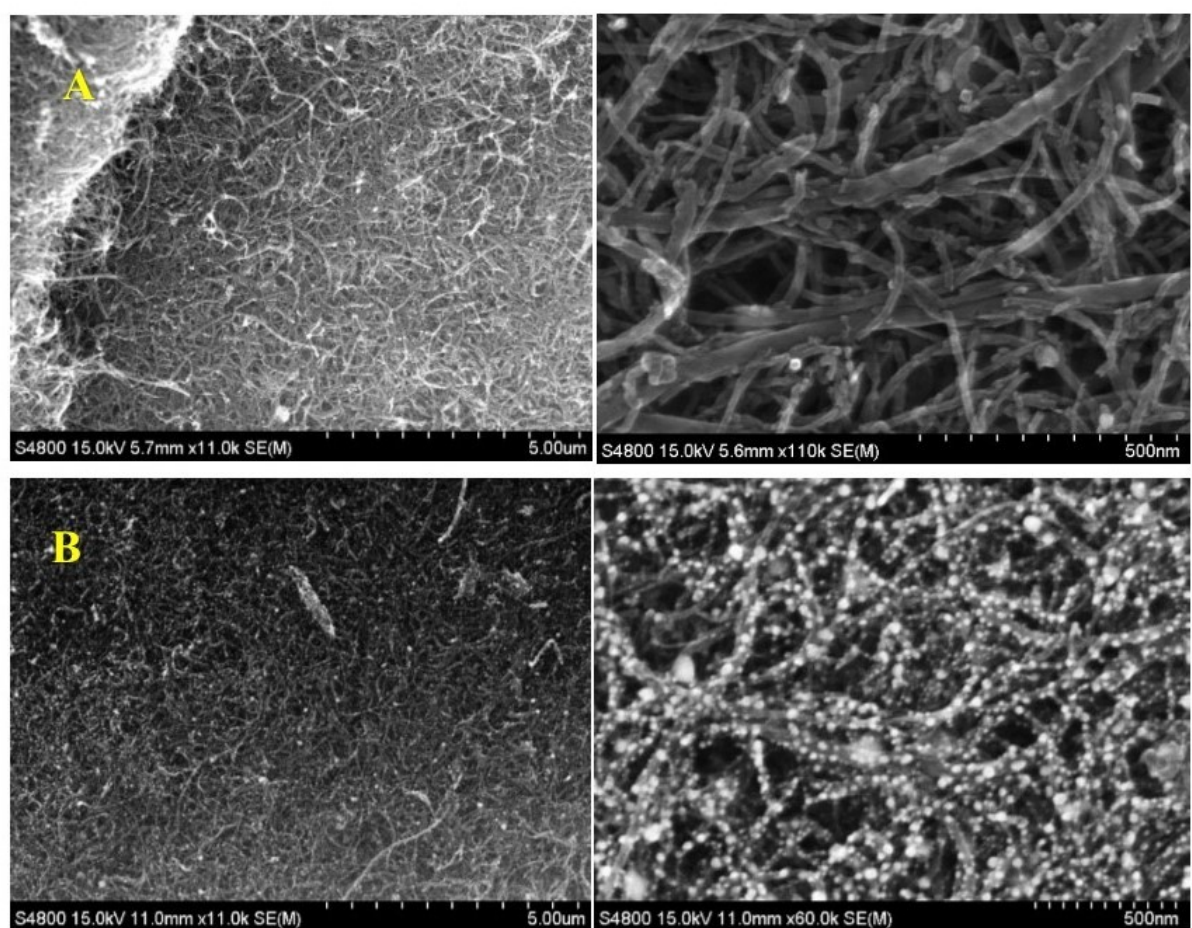

Figure 5.9: $5 \mu \mathrm{m}$ (Left) and $500 \mathrm{~nm}$ (Right) SEM images for (A) 10HNS and (B) 20HNS modulated by microwave irradiation.

The EDX spectra confirmed the corresponding metal, oxygen, and carbon for all HNS. The characteristic energy level for $\mathrm{O}_{2}$ and $\mathrm{C}$ are located at $0.54 \mathrm{eV}$ and $0.2 \mathrm{eV}$, respectively. While for the metals peaks were found to be at $(3 \mathrm{eV}),(0.35 \mathrm{eV}$ and 4.5 $\mathrm{eV}),(1.5 \mathrm{eV})$ and $(0.7 \mathrm{eV}, 6.4 \mathrm{eV}$ and $7.1 \mathrm{eV})$ for $\mathrm{Ag}, \mathrm{Ti}, \mathrm{Al}$ and $\mathrm{Fe}$, respectively. Finally, the results agreed with those obtained from both TGA (Section 5.3.5) and theoretical calculation in terms of compositions (Section5.2.2). 
Chapter Five - Fabrication and characterization of versatile metal/metal oxide carbon based hybrid nanostructures.

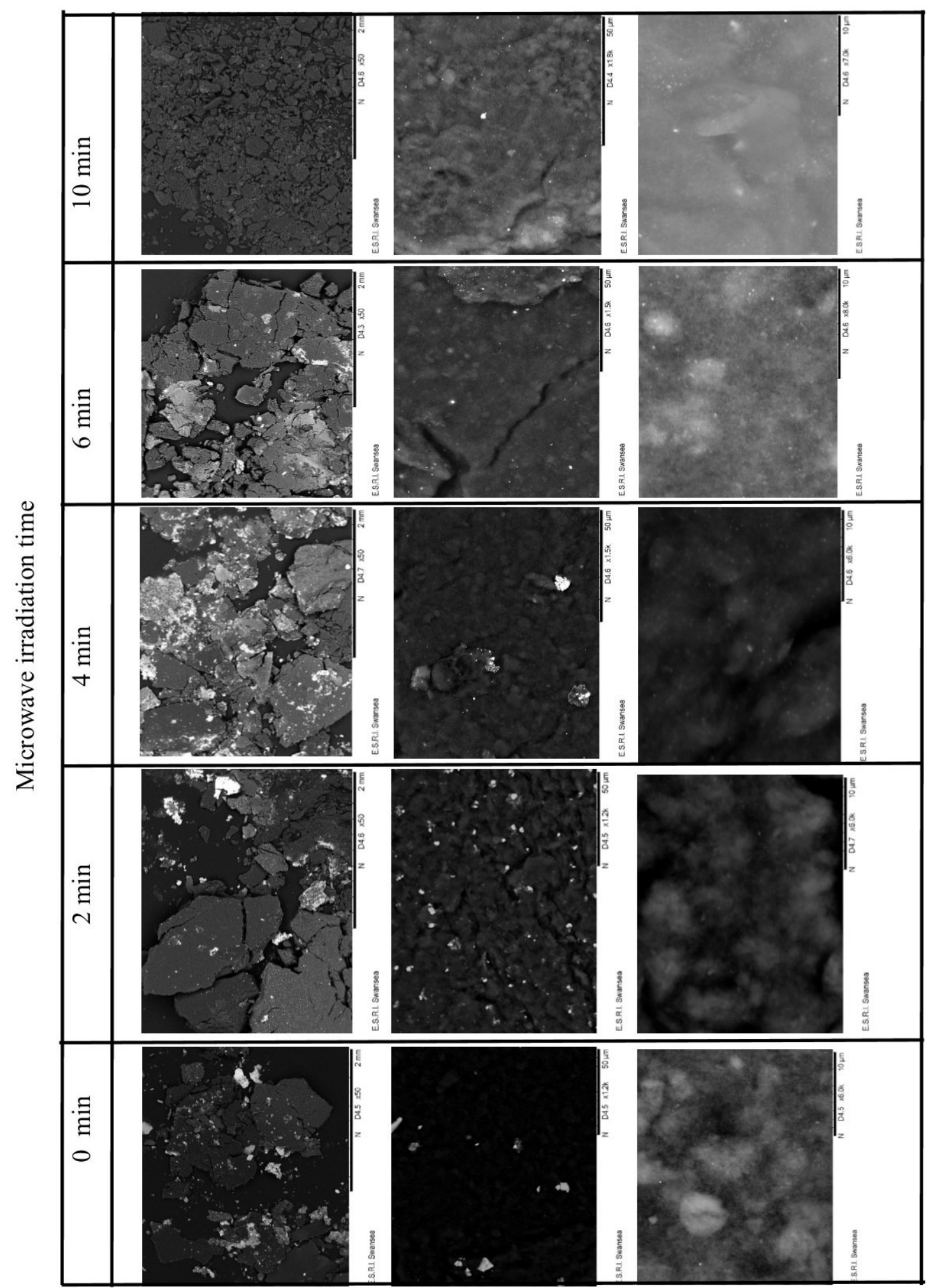

Figure 5.10: $2 \mathrm{~mm}, 50 \mu \mathrm{m}$ and $10 \mu \mathrm{m}$ SEM images for $10 \mathrm{HNS}$ synthesised under different microwave irradiation time. 
Chapter Five - Fabrication and characterization of versatile metal/metal oxide carbon based hybrid nanostructures.
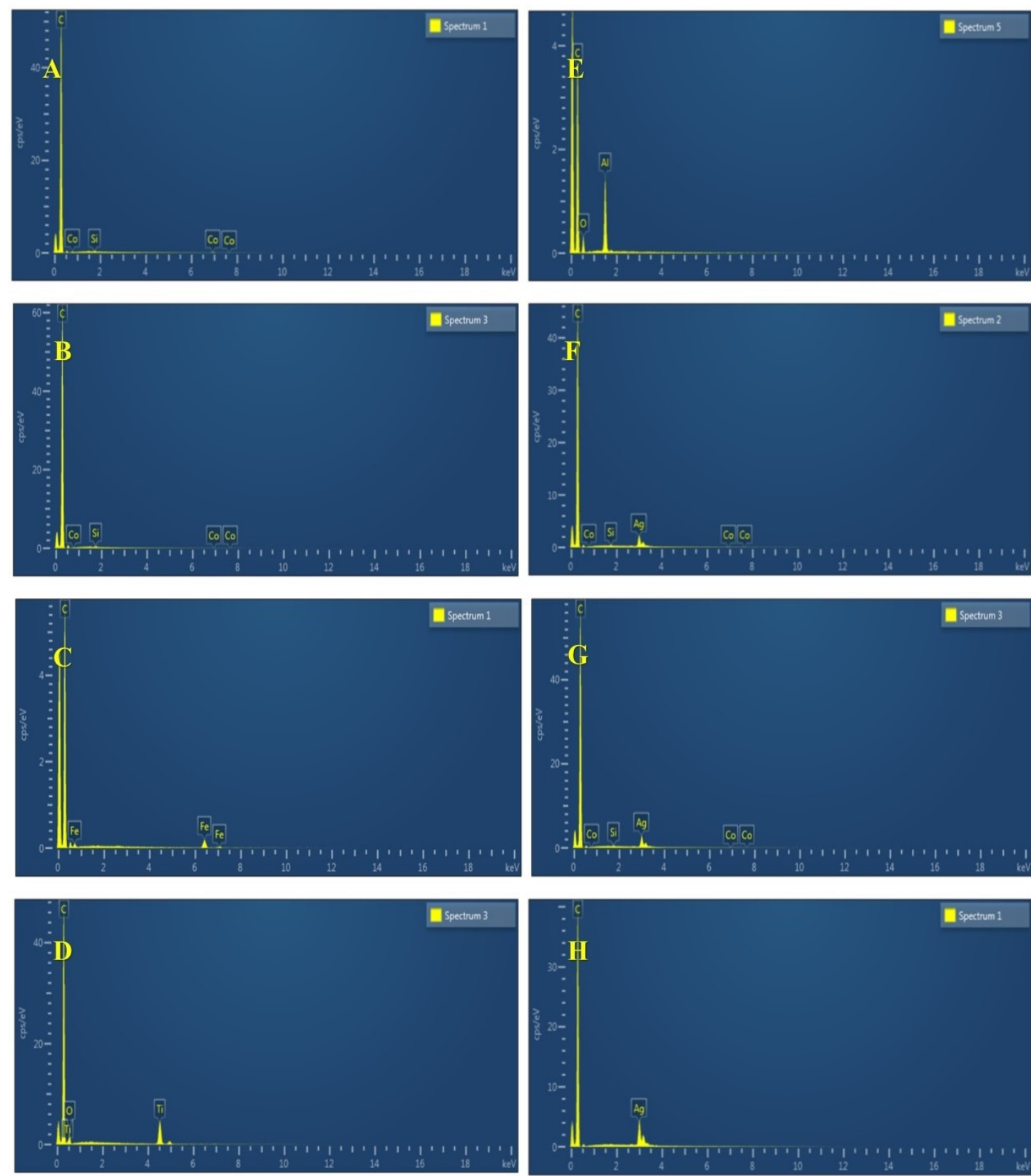

Figure 5.11: EDX analysis for (A) p-MWCNTs, (B) f-MWCNTs, (C) $\mathrm{Fe}_{2} \mathrm{O}_{3}$-CNTs, (D) $\mathrm{TiO}_{2}$-CNTs, (E) $\mathrm{Al}_{2} \mathrm{O}_{3}$-CNTs and (F) Ag-CNTs. (G) 10HNS, and (H) 20HNS

\subsubsection{Thermal behaviour analysis (TGA)}

TGA analysis was conducted to illustrate the thermal decomposition behaviour of various samples, and to confirm the theoretical amount of M/MO loading weight used to coat the nanotubes. The initial, main and char decomposition regions of TGA curves are illustrated in (Figure 5.12). The slight initial weight loses detected below the onset decomposition temperature of samples are ascribed to the ambient moisture content in the samples or evolution of functional groups (Yudianti et al., 2011). In fact, the 
Chapter Five - Fabrication and characterization of versatile metal/metal oxide carbon based hybrid nanostructures.

significant change in the thermal properties and weight loss under an oxidative environment was different for each sample. A gradual slight weight loss (9.2\%) was noticed for f-MWCNTs before the significant loss at $551{ }^{\circ} \mathrm{C}$. This could be linked to the decomposition of the thermally unstable organic functional moieties in the functionalized sample (Vuković et al., 2009). Compared to f-MWCNTs, p-MWCNTs were distinctly more stable and only $2.2 \%$ weight loss was detected with a lower decomposition start temperature $\left(467^{\circ} \mathrm{C}\right)$, which could be attributed to the variation in the metal catalyst residues and graphitisation degree before and after acid treatment (Chen et al., 2004). The presence of metal catalyst residues was decreased after acid treatment, from $2.3 \mathrm{wt} . \%$ to $0.3 \mathrm{wt} . \%$, indicating further purification was accomplished. However, the acid treatment also caused defect sites such as sidewall damages and ring end openings, and hence to an increase in the surface area of the nanotubes (Kundu et al., 2008).

Interestingly, Ag deposition on the nanotubes' side walls significantly reduced the onset decomposition temperature for both 10HNS and 20HNS to $309{ }^{\circ} \mathrm{C}$ and $266{ }^{\circ} \mathrm{C}$, respectively (Figure 5.12). Ag is known as functional oxidative catalyst able to catalyse the low-temperature oxidation of nanotubes under $\mathrm{O}_{2}$ atmosphere (Alimohammadi et al., 2012). Amount of Ag, after decomposition of 10HNS and 20HNS, has confirmed the theoretical loading level used in this work and was found to be $10.5 \%$ and $20.2 \%$, respectively. Similarly, this initial wet loss was also observed for the other HNS and could be due to the condensation reactions of metal-OH residual groups presented at the HNS materials (Amais et al., 2007). However, the presence of $\mathrm{TiO}_{2}$ and $\mathrm{Fe}_{2} \mathrm{O}_{3}$ did not have any influence on the thermal properties of the MWCNTs, which were almost identical to that of $f$-MWCNTs (Figure 5.13). Unsurprisingly, this was not the case for the nanotubes coated with $\mathrm{Al}_{2} \mathrm{O}_{3}$, and the thermal decomposition temperature commenced at $635{ }^{\circ} \mathrm{C}$ due to the improved thermal stability of MWCNTs resulting from the synergy of alumina. Finally, the amount of $\mathrm{M} / \mathrm{MO}$, remaining after decomposition of nanotubes in the samples, has confirmed the theoretical loading level adopted in this research. 
Chapter Five - Fabrication and characterization of versatile metal/metal oxide carbon based hybrid nanostructures.

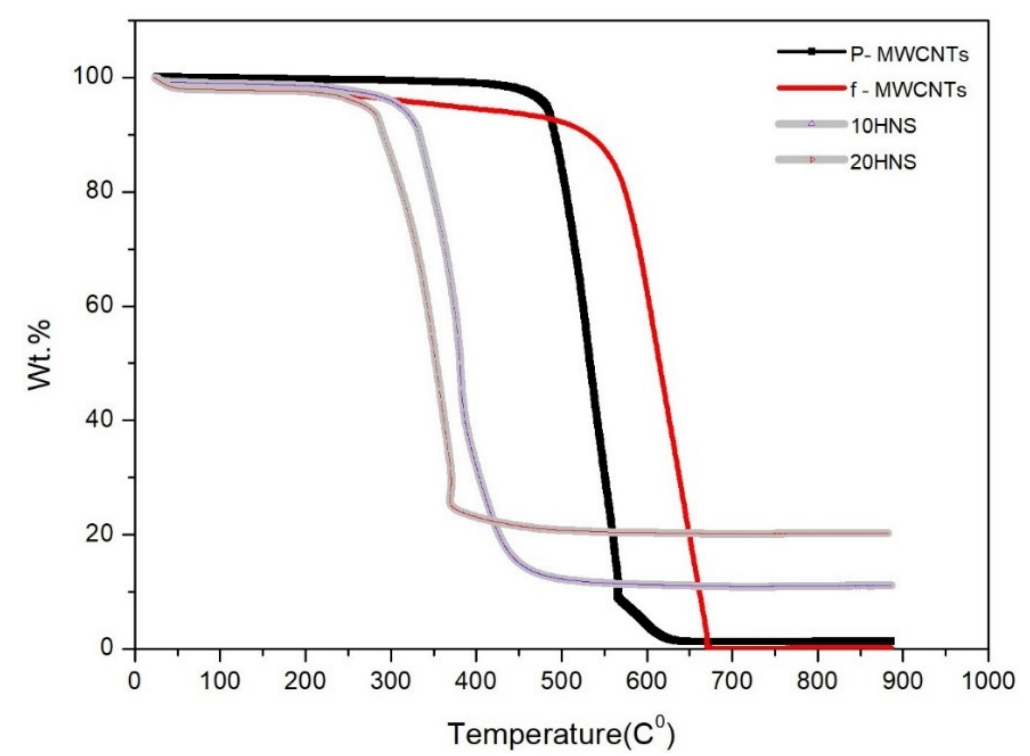

Figure 5.12: Thermogravimetric curves of p-MWCNT, f-MWCNTs, 10HNS and 20HNS.

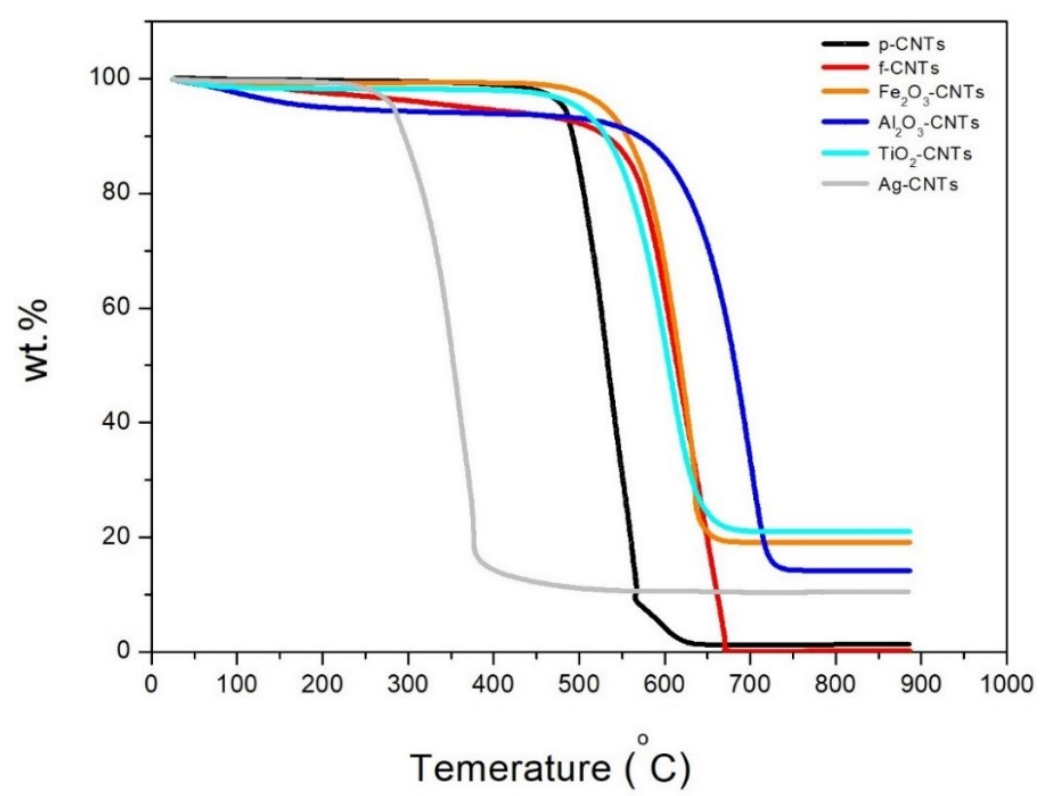

Figure 5.13: TGA curves in the air for $\mathrm{M} / \mathrm{MO}$ nanocomposites

\subsubsection{Raman spectroscopy}

Raman spectroscopy, as a non-destructive characterisation technique, has been commonly employed in the field of chemistry and material science for direct identification/interpretation of different molecules, with minimum sampling handling 
Chapter Five - Fabrication and characterization of versatile metal/metal oxide carbon based hybrid nanostructures.

issues (Hilal et al., 2017). The structural changes, prior and after decorating the nanotubes with M/MO, were determined by Raman spectroscopy bands, Figure 5.14. All carbon nanotubes bands differentiated by Raman spectra were located between $1000 \mathrm{~cm}^{-1}$ and $1700 \mathrm{~cm}^{-1}$. The band denoted as (G-band) at $1572 \mathrm{~cm}^{-1}$ is associated with the graphitic layers and is the characteristic feature of all $\mathrm{sp}^{2}$ hybridised carbon allotropes (Baro et al., 2013). The other additional band (D-band) at $1340 \mathrm{~cm}^{-1}$ identifies the defects/disorders in the $\mathrm{sp}^{2}$ hybridised carbon, which could be impurities, lattice distortions in the nanotubes, $\mathrm{sp}^{3}$ hybridised bonds, and/or existing functional groups created after acid treatment (Gupta \& Saleh, 2011). While the mode at 2685 $\mathrm{cm}^{-1}$ was attributed to the first overtone of the band at D and is denoted as $\mathrm{G}^{\prime}$ mode (Vinayan et al., 2012). Following the acid treatment and the subsequent decoration step, some differences between the spectra have been highlighted; a decrease in the $G$ band intensity relative to the $\mathrm{D}$ band have been observed for f-CNTs and all other HNS. The intensity ratio between $\mathrm{D}$ band and $\mathrm{G}$ band $\left(I_{D} / I_{G}\right)$ was used to determine the degree of disorder in the samples after processing with $\mathrm{HNO}_{3}$ and further decoration with $\mathrm{M} / \mathrm{MO}$. An increment in the $I_{D} / I_{G}$ from 0.76 to 0.85 was observed, suggesting an increase in the defects and disorder on the nanotubes after processing with $\mathrm{HNO}_{3}$ has been accomplished. Furthermore, the presence of metal/metal oxides on the surface of nanotubes caused explicit changes in Raman scattering intensity, and the $I_{D} / I_{G}$ were found to be 0.90, 0.85, 0.85, 0.92 for $\mathrm{Fe}_{2} \mathrm{O}_{3}-\mathrm{CNTs}, \mathrm{Al}_{2} \mathrm{O}_{3}-\mathrm{CNTs}$, $\mathrm{TiO}_{2}-\mathrm{CNTs}$, and AgCNTs, respectively. This indicates that incorporating $\mathrm{M} / \mathrm{MO}$ induced more defects and degree of disorder of the CNTs. Even though the change in the $I_{D} / I_{G}$ ratio has been used as an indication for the attachment of metallic NPs on the surface of nanotubes (Baro et al., 2013), this change was not tangible after deposition of metallic NPs in this work. This could be due to the preferential growth of NPs on surface defects sites of nanotubes, resulting in a reduction in these structural defects, and leading to a small increase in the $I_{D} / I_{G}$ ratio indeed (Lin et al., 2011; Lin et al., 2009). Moreover, different nanocomposites manifested a morphologic $I_{D} / I_{G}$ ratio, regardless if the same f-CNTs were used for the later metal deposition step. Probably, this disparity may be different from one metal to another depending on NPs size and distribution's homogeneity, when grown on surface defects of the nanotubes. 
Chapter Five - Fabrication and characterization of versatile metal/metal oxide carbon based hybrid nanostructures.

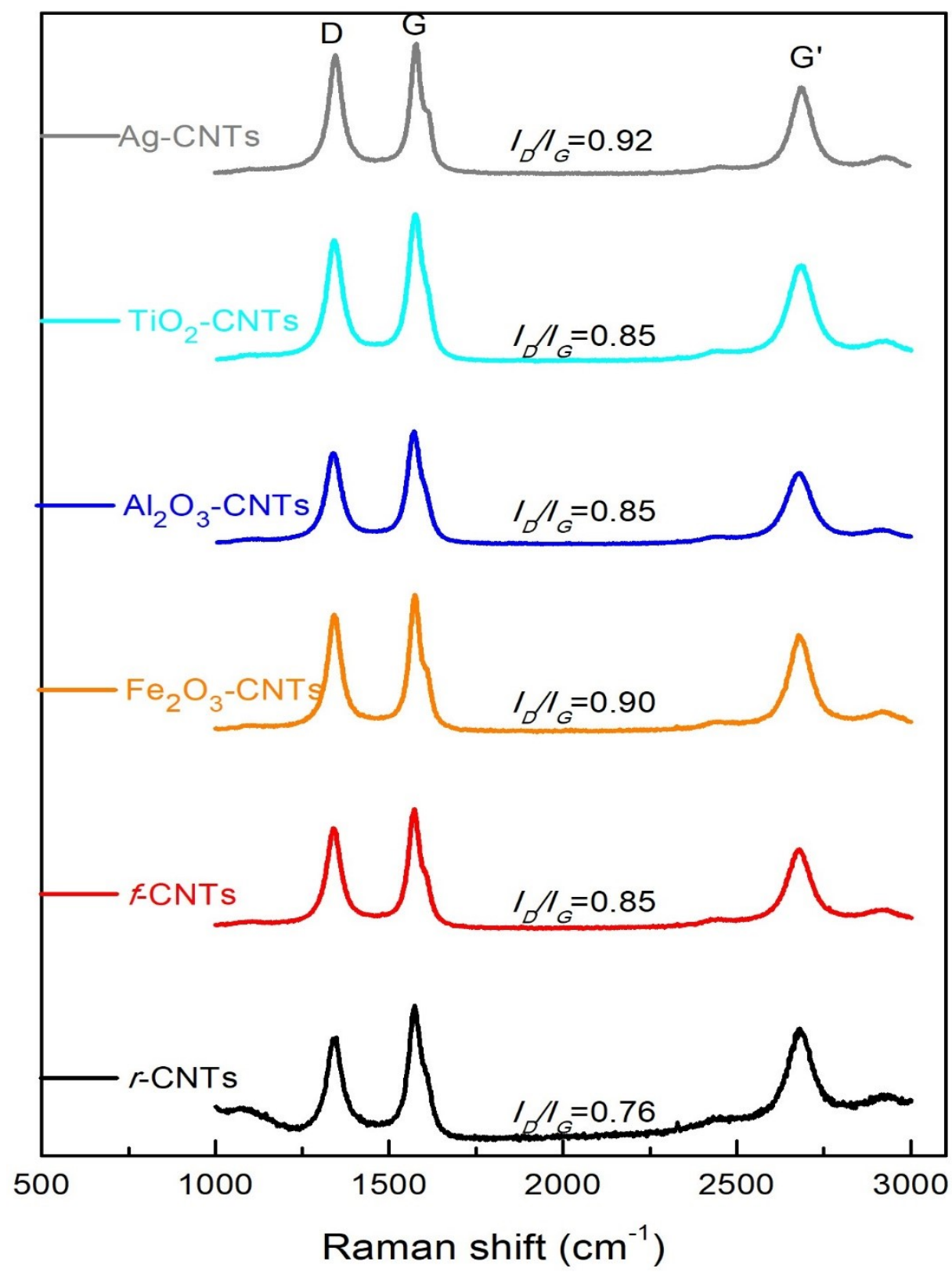

Figure 5.14: Raman spectroscopy results for pristine, functionalized and metal/metal oxides decorated MWCNTs, $\left(1000 \mathrm{~cm}^{-1}-3000 \mathrm{~cm}^{-1}\right)$.

In Raman spectroscopy, different material phases can be distinguished, depending on their individual frequency, and elucidated as peaks. Figure 5.15 shows the Raman spectra of different metallic NPs loaded on nanotubes between $100 \mathrm{~cm}^{-1}-1000 \mathrm{~cm}^{-1}$. The well-defined Raman peak of $\mathrm{TiO}_{2}$ determined at $150 \mathrm{~cm}^{-1}$ is assigned to the main anatase vibration mode $\left(E_{g}\right)$ of titanium. Other Vibration peaks observed at $398 \mathrm{~cm}^{-1}$, $505 \mathrm{~cm}^{-1}, 625 \mathrm{~cm}^{-1}$ represent $\left(\mathrm{B} 1_{\mathrm{g}}\right),\left(\mathrm{A} 1_{\mathrm{g}}\right)$ and $\left(\mathrm{E}_{\mathrm{g}}\right)$ suggesting that anatase phase is the main crystallite structure for the $\mathrm{TiO}_{2}$ (Falaras et al., 2000). While the scattering peaks 
Chapter Five - Fabrication and characterization of versatile metal/metal oxide carbon based hybrid nanostructures.

located at $216 \mathrm{~cm}^{-1}$ and $282 \mathrm{~cm}^{-1}, 393 \mathrm{~cm}^{-1}$ and $616 \mathrm{~cm}^{-1}$ in the $\mathrm{Fe}_{2} \mathrm{O}_{3}$ Raman spectrum are attributed to the vibration modes of $\alpha-\mathrm{Fe}_{2} \mathrm{O}_{3}$ (Gund et al., 2015), which agreed with the suggested XRD results.

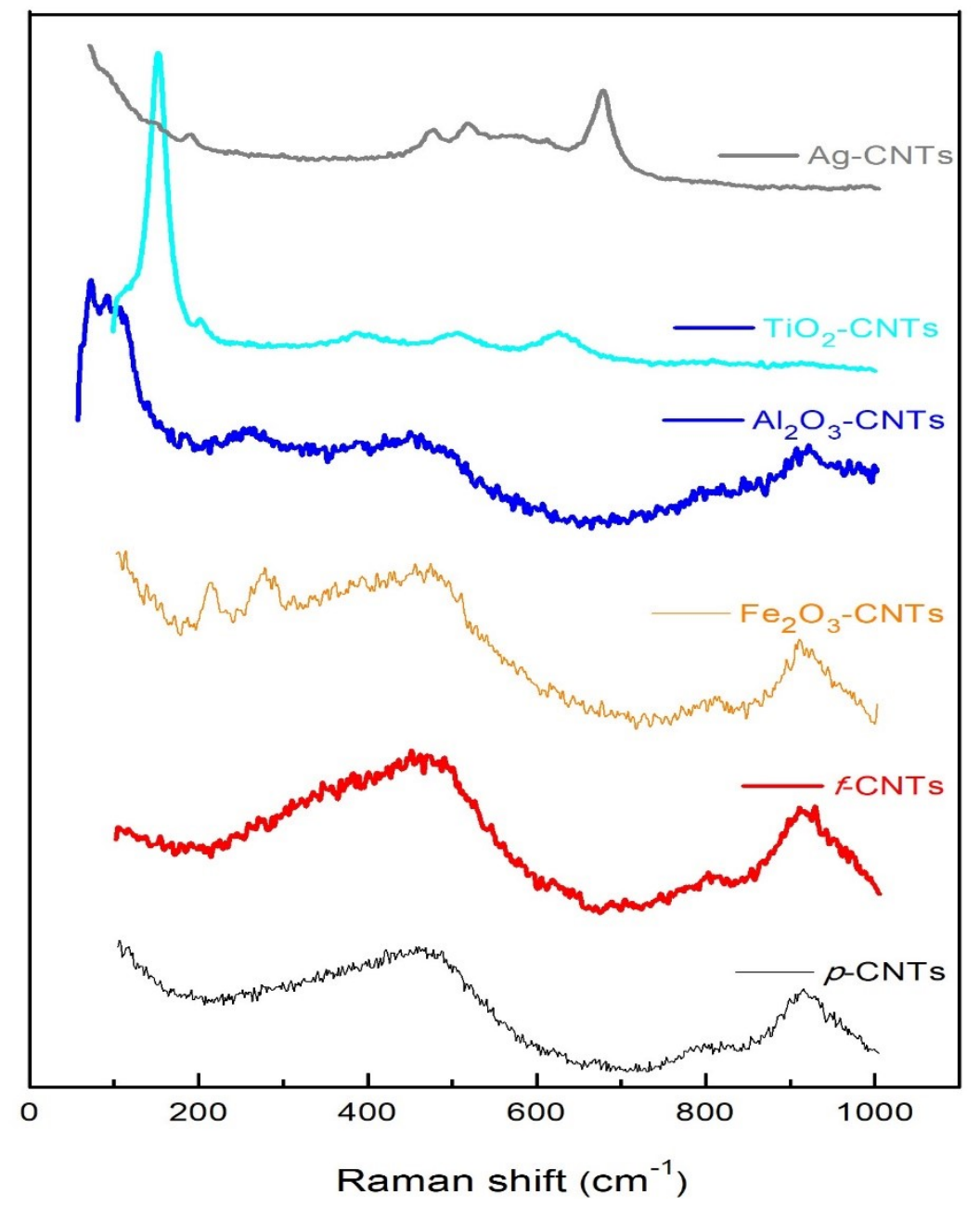

Figure 5.15: Raman spectroscopy results for pristine, functionalized and metal/metal oxides decorated MWCNTs, $(100 \mathrm{~cm}-1-1000 \mathrm{~cm}-1)$

For the HNS modulated with microwave irradiation (10HNS and 20HNS), the $\left(I_{G} / I_{D}\right)$ was found to be 0.89 and 1.027 for $10 \mathrm{HNS}$ and 20HNS (Figure 5.16A), respectively. The changes in the intensities of the bands and $\left(I_{G} / I_{D}\right)$ evidenced further changes were also found to be induced into Raman scatter intensity after silver deposition, which is a substantiation for Ag-NPs attachment. This change was more remarkable in the 20HNS than the 10HNS sample. Furthermore, a more defined peak on the right side of the G-band was evolved around $1620 \mathrm{~cm}^{-1}$ (D'-band) after pMWCNTs processing, (Figure 5.16B). This evolution of the peak was ascribed to functionalization and later on to deposition of $\mathrm{Ag}$ on the surface of nanotubes (Travessa 
Chapter Five - Fabrication and characterization of versatile metal/metal oxide carbon based hybrid nanostructures.

et al., 2014). Herein, it should be noted that the smaller AgNPs could preferentially grow on the surface defects of nanotubes, which explain the small overall increase in $I_{G} / I_{D}$ after decorating the MWCNTs (Lin et al., 2011; Lin et al., 2009). While in the case of $20 \mathrm{HNS}$, the defects/disorders were more significant. These results are in agreement with those obtained from XRD measurements.
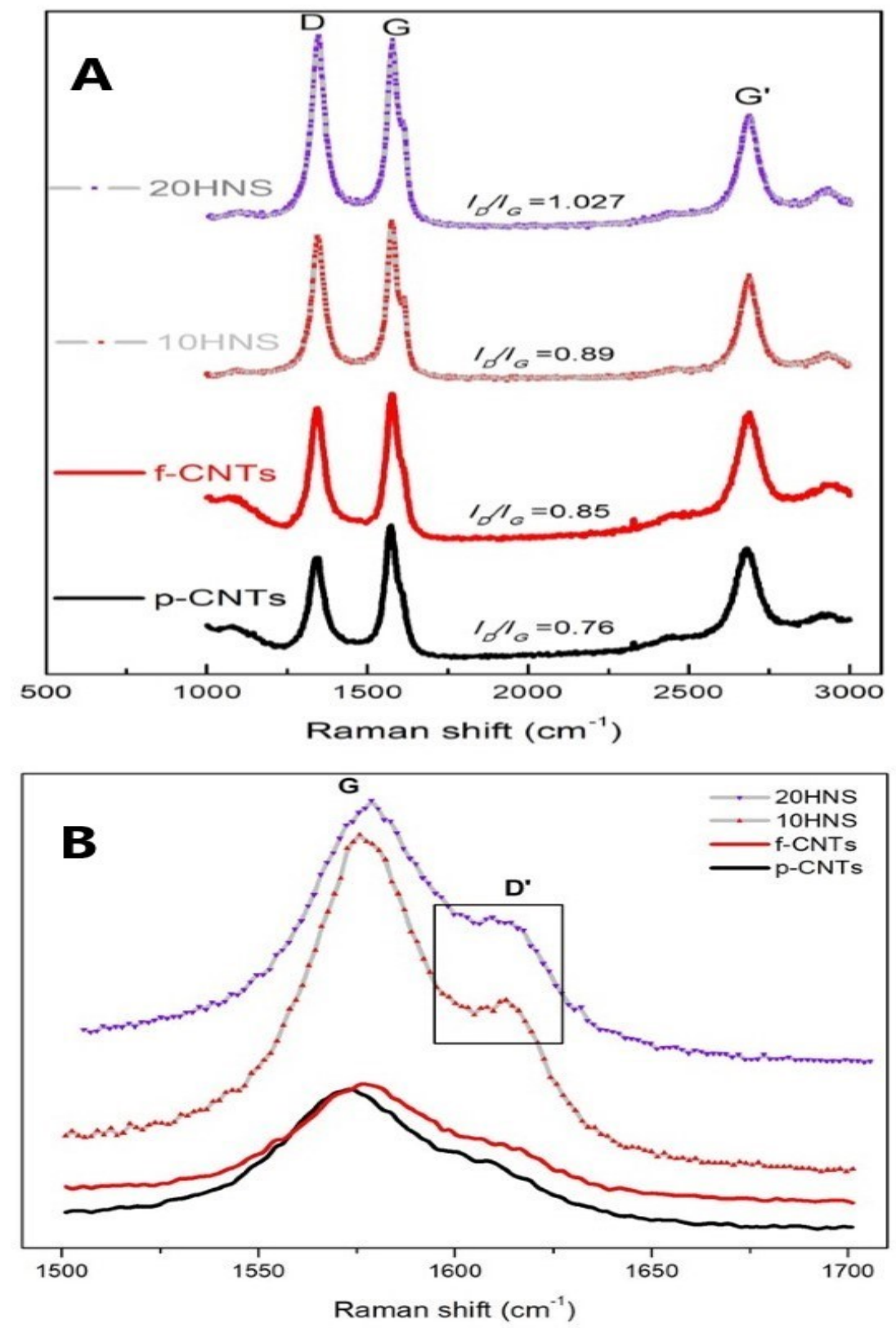

Figure 5.16: Raman spectroscopy results for pristine, functionalized 10HNS and 20HNS, (A) $1000-3000 \mathrm{~cm}^{-1}$, and (B) $1500-1700 \mathrm{~cm}^{-1}$. 
Chapter Five - Fabrication and characterization of versatile metal/metal oxide carbon based hybrid nanostructures.

\subsubsection{X-ray photoelectron spectroscopy (XPS)}

XPS is a standard surface analysis instrument for a broad range of materials, where valuable information on their chemical state and composition can be obtained (Hilal et al., 2017). To reveal the chemical composition and oxidation state of the uppermost surface of HNS, a typical XPS survey scan has been implemented. The elemental composition of nanotubes, including carbon (C) and oxygen (O), were observed in scans of all samples. The photoelectron peak $\mathrm{C} 1 \mathrm{~s}$, ascribed to the graphitic carbon on nanotubes, is located at $284.8 \mathrm{eV}$. And the other elemental characteristic peak (O1s) is located at $532 \mathrm{eV}$ binding energy (BE), as shown in Figure 5.17 - Figure 5.21.

Following the acid treatment, a change in $\mathrm{C} 1 \mathrm{~s}$ and $\mathrm{O} 1 \mathrm{~s}$ has been observed indicating the degree of surface oxidation ( Figure 5.17). The $\mathrm{O} / \mathrm{C}$ ratio has increased from $7 \%$ to $9.65 \%$, indicating more oxygen atoms are bounded to the f-MWCNTs according to XPS results. These results correlated with the increased defects and disorders $\left(I_{D} / I_{G}\right.$ ratio $)$ created on the nanotubes surface after acid treatment, which was determined by Raman spectra. More quantitative information about the surface functional groups, generated during the acid treatment, were achieved by highresolution fitted curve XPS spectra over $\mathrm{C} 1 \mathrm{~s}$ regions of as received and functionalized nanotubes. The deconvolution of XPS spectra is shown in Figure 5.17B and Figure 5.17C. The C1s spectra were resolved into seven sub-bands that evidence carbon $\mathrm{sp}^{2}$ structure $(\mathrm{BE}=284.5 \pm 0.2), \mathrm{sp}^{3}$ structure $(\mathrm{BE}=285.4 \pm 0.15)$, alcohol or phenol groups $(\mathrm{BE}=286.5 \pm 0.2)$, quinone or carbonyl groups $(\mathrm{BE}=287.4 \pm 0.2)$, ester or carboxylic acid groups $(\mathrm{BE}=288.7 \pm 0.1)$, carbonate groups $(\mathrm{BE}=289.7 \pm 0.2)$, and satellite peaks $(\mathrm{BE}=291.2 \pm 0.1)$. These data are consistent with those found in the literature (Gong et al., 2013). A significant increase in the amount of -COO groups was observed in functionalized samples at $(\mathrm{BE}=288.7)$, including carboxyl and anhydrides. Signals of oxygen $(\mathrm{O} 1 \mathrm{~s})$ are distinguished as single $(\mathrm{BE}=531.2 \pm 0.1)$ and double $(\mathrm{BE}=531.6 \pm 0.1)$ bonded oxygen with carbon ( Figure 5.17E and Figure 5.17F). 
Chapter Five - Fabrication and characterization of versatile metal/metal oxide carbon based hybrid nanostructures.
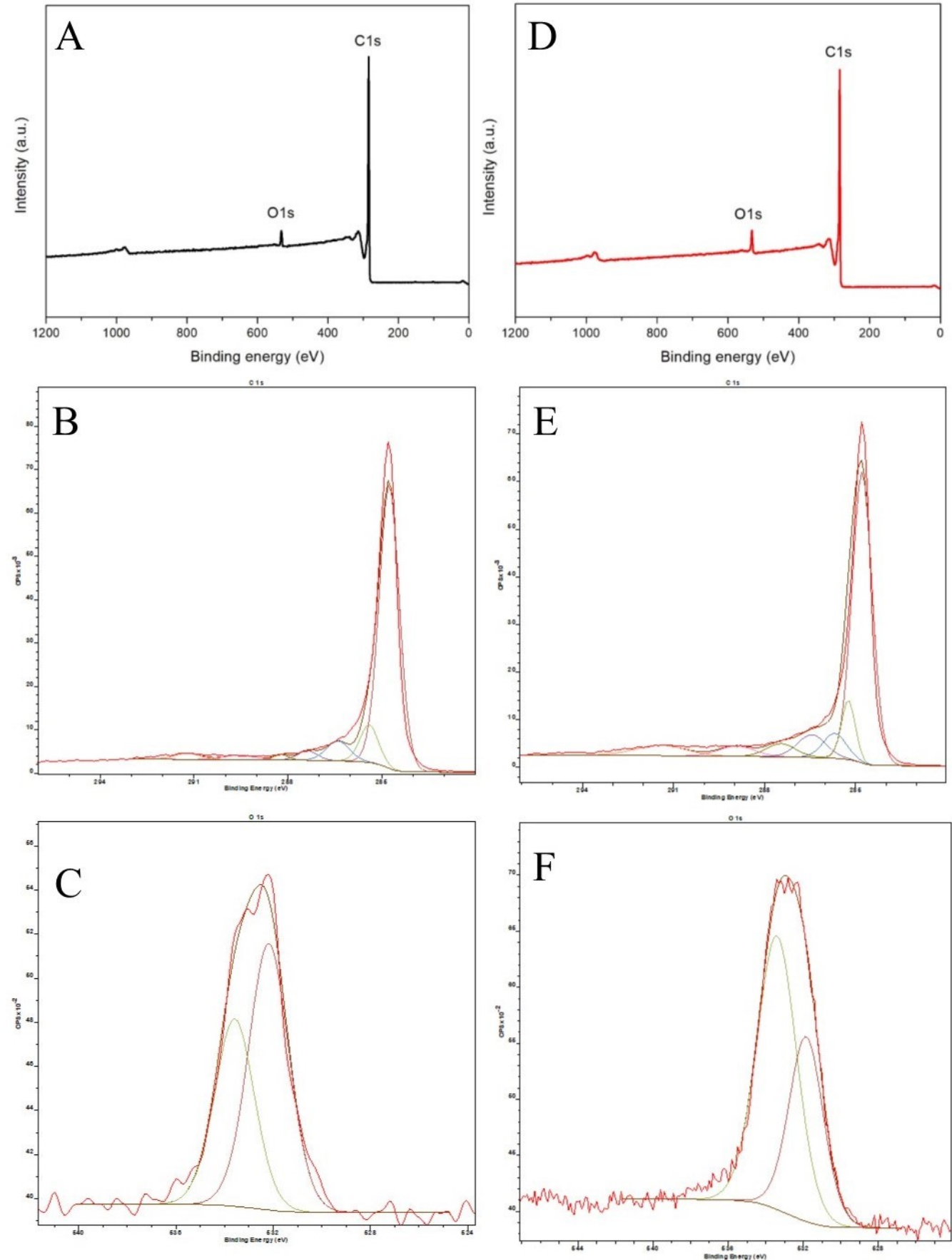

Figure 5.17: Full XPS spectra, $\mathrm{C}$ 1s and $\mathrm{O}$ 1s peak deconvolution of as-received CNTs (A, B and C), and $\mathrm{HNO}_{3}$-treated MWCNT (D, E, and F) samples.

The subsequent decoration of nanotubes with $\mathrm{M} / \mathrm{MO}$ has generated novel peaks in the XPS spectra. The XPS survey spectrum evidenced the coexistence of iron, carbon, and oxygen in the nanocomposite. The full $\mathrm{Fe}_{2} \mathrm{O}_{3}-\mathrm{CNTs}$ spectrum is depicted in Figure 5.18A. While Figure 5.18B illustrates the shape and position of peaks at $711.3 \mathrm{eV}$ and 
Chapter Five - Fabrication and characterization of versatile metal/metal oxide carbon based hybrid nanostructures.

$724.7 \mathrm{eV}$ and assigned to $\mathrm{Fe} 2 \mathrm{p}_{3 / 2}$ and $\mathrm{Fe} 2 \mathrm{p}_{1 / 2}$ of $\alpha-\mathrm{Fe}_{2} \mathrm{O}_{3}$, respectively (Warwick et al., 2015). The energy separation between the two spin-orbit components $(\Delta \mathrm{BE}=13.4 \mathrm{eV})$ confirmed the formation of iron (III) oxide, free from other oxidation states (Carraro et al., 2015). In addition, there was a shake-up satellite peak situated at $719 \mathrm{eV}$, which is identified as the characteristic of $\mathrm{Fe}^{3+}$ in $\mathrm{Fe}_{2} \mathrm{O}_{3}$ and no other $\mathrm{Fe}^{2+}$ state (Wu et al., 2015). Deconvolution of C 1s and O 1s peak are illustrated in Figure 5.18C and Figure 5.18D, respectively. The $\mathrm{C} 1 \mathrm{~s}$ spectrum can be fitted into three peaks at $\mathrm{BE}=284.6 \mathrm{eV}$, $\mathrm{BE}=286.2 \mathrm{eV}$ and $\mathrm{BE} 288.9 \mathrm{eV}$ ascribed to $(\mathrm{C}-\mathrm{C}),(\mathrm{C}-\mathrm{O})$ and $(\mathrm{C}=\mathrm{O})$, respectively $(\mathrm{Li}$ $\& \mathrm{He}, 2017)$. While the O1s peak was deconvoluted by two bands at $\mathrm{BE}=530.1 \pm 0.1$ (ascribed to lattice oxygen in $\mathrm{Fe}_{2} \mathrm{O}_{3}$ ) and $\mathrm{BE}=532 \pm 0.2$ (represented the surface
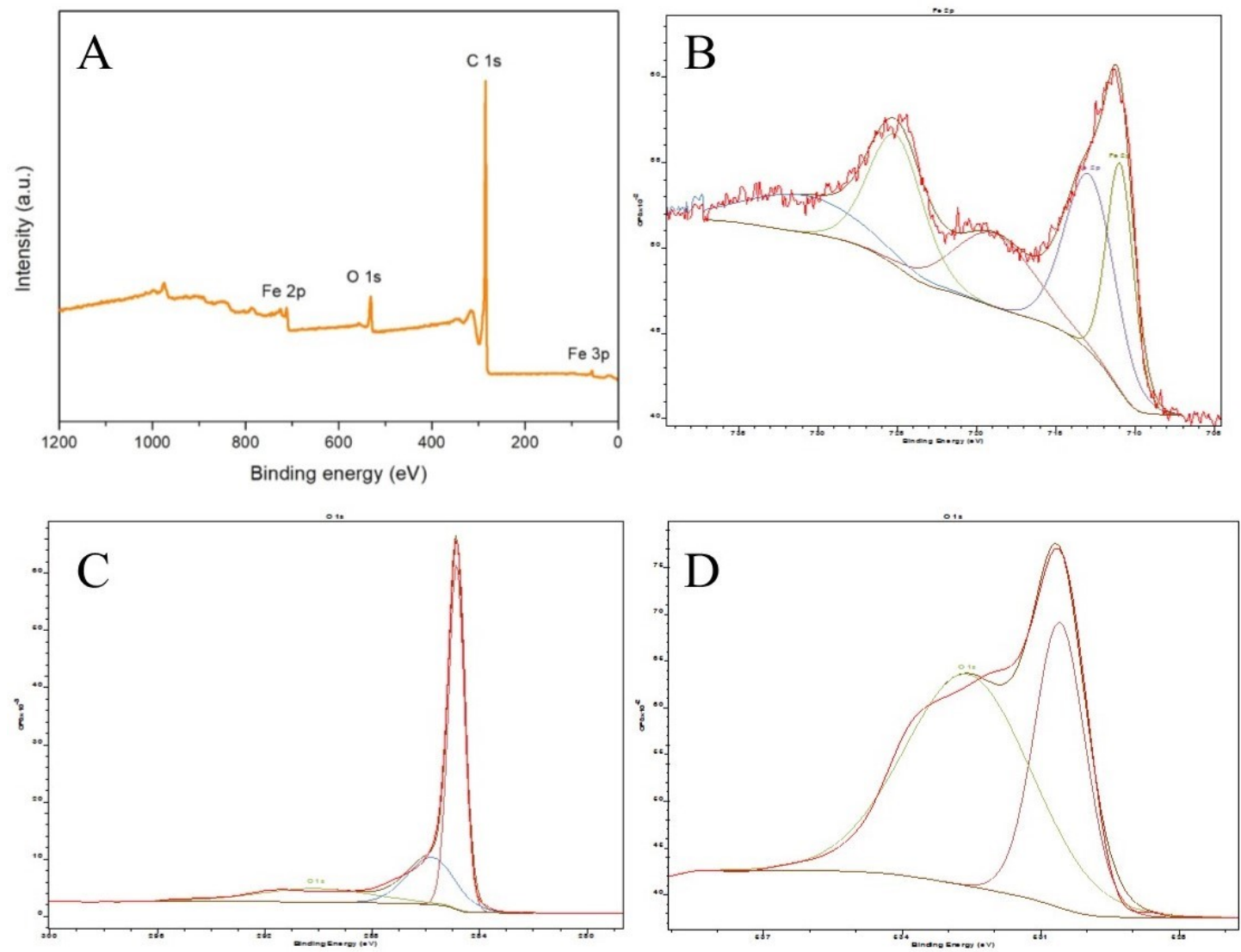

hydroxyl/carbonates) (Carraro et al., 2015).

Figure 5.18: Full XPS spectra (A), Fe 2p (B), C 1s (C) and O 1s (D) peak deconvolution of $\mathrm{Fe}_{2} \mathrm{O}_{3} \mathrm{CNTs}$.

The phase of $\mathrm{TiO}_{2}$ in the $\mathrm{TiO}_{2} \mathrm{CNTs}$ nanocomposite was also verified by XPS survey spectra, Figure 5.19. The full survey spectrum shown in Figure 5.19A is composed of sharp signals due to $\mathrm{C}$, Ti and $\mathrm{O}$ at $\mathrm{BE}=284.8 \mathrm{eV}, 459.6 \mathrm{eV}$ and $531 \mathrm{eV}$ 
Chapter Five - Fabrication and characterization of versatile metal/metal oxide carbon based hybrid nanostructures.

respectively. The core level $\mathrm{BE} 459.6 \mathrm{eV}$ and $465.3 \mathrm{eV}$ of $\mathrm{Ti} 2 \mathrm{p}_{3 / 2}$ and $\mathrm{Ti} 2 \mathrm{p}_{1 / 2}$ respectively, have indicated the formation of $\mathrm{Ti}^{+}$in titanium (Koli et al., 2017), with $5.7 \mathrm{eV}$ splitting energy between them (Figure 5.19B). This is in agreement with those of neat $\mathrm{TiO}_{2}$ and clearly indicates that all $\mathrm{Ti}$ cations in the nanocomposites are in the oxidation state IV (Wang et al., 2008). The C 1s spectrum (Figure 5.19C) is deconvoluted into three peaks. The sharp peak (at $\mathrm{BE}=284.8 \mathrm{eV}$ ) and the broad peak (at $\mathrm{BE}=286 \mathrm{eV}$ ) are assigned to $\mathrm{sp}^{2}$ and $\mathrm{sp}^{3}$ carbon, respectively. While the third peak at $\mathrm{BE}=289 \mathrm{eV}$ is ascribed to $\mathrm{O}=\mathrm{C}-\mathrm{O}$ carboxyl carbon (Hemalatha et al., 2014; Li et al., 2007a).The corresponding $\mathrm{O} 1 \mathrm{~s}$ region is illustrated in Figure 5.19D and is deconvoluted into two peaks. The sharp peak at $\mathrm{BE}=530.5 \mathrm{eV}$ is ascribed to the lattice oxygen in the anatase phase of $\mathrm{TiO}_{2}$ while the other peak at $\mathrm{BE}=532 \mathrm{eV}$ corresponds to $\mathrm{C}=\mathrm{O}$ or $\mathrm{Ti}-\mathrm{O}-\mathrm{H}(\mathrm{Li}$ et al., 2007a).
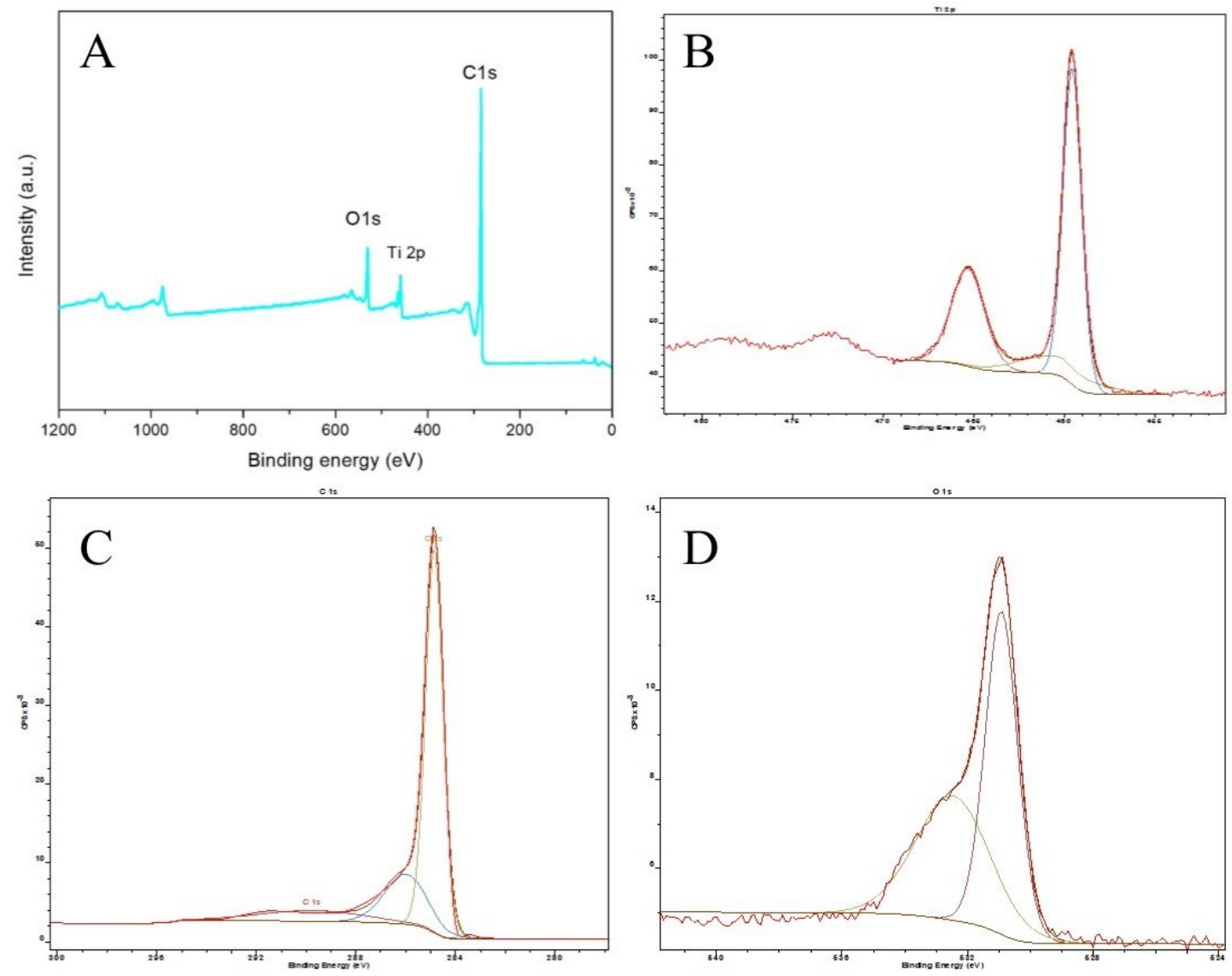

Figure 5.19: Full XPS spectra (A), Ti 2p (B), C 1s (C) and O 1s (D) peak deconvolution of $\mathrm{TiO}_{2} \mathrm{CNTs}$.

The full XPS spectrum of $\mathrm{Al}_{2} \mathrm{O}_{3}$ CNTs was illustrated in Figure 5.20A. Formation of $\mathrm{Al}_{2} \mathrm{O}_{3}$ on the surface of nanotubes was asserted by the well resolved characteristic 
Chapter Five - Fabrication and characterization of versatile metal/metal oxide carbon based hybrid nanostructures.

peak $\mathrm{Al} 2 \mathrm{p}$ detected at a binding energy position of $74.6 \mathrm{eV}$, which agrees with corresponding BE reported in the literature (Reddy et al., 2014), and shown in Figure 5.20B. Similar to other MO coated MWCNTs, the C1s spectra (Figure 5.20C) has deconvoluted into three signals represents the $\mathrm{sp}^{2}$ carbon $(\mathrm{BE}=284.8 \mathrm{eV}), \mathrm{C}-\mathrm{O}$ $(\mathrm{BE}=285.8 \mathrm{eV})$ and $\mathrm{C}=\mathrm{O}(\mathrm{BE}=288.8 \mathrm{eV})$. While the fairly broad $\mathrm{O}$ 1s spectrum (528 $\mathrm{eV}-537 \mathrm{eV}$ ) refers to the presence of different oxygen bearing species (Figure 5.20D). The peak at $\mathrm{BE}=531.3 \mathrm{eV}$ suggested the formation of oxides related to oxygen $\left(\mathrm{O}_{\mathrm{o}}\right)$ while the peak manifested at $\mathrm{BE}=532.1 \mathrm{eV}$ indicated the presence of chemosorbed oxygen $\left(\mathrm{O}_{\mathrm{a}}\right)$ (Reddy et al., 2014). The difference between the BE of Al $2 \mathrm{p}$ and O1sbonded $(456.8 \pm 0.2 \mathrm{eV})$ was in agreement with $\mathrm{O}-\mathrm{Al}-\mathrm{O}$ bonding in $\mathrm{Al}_{2} \mathrm{O}_{3}$, while the obtained difference in $\mathrm{BE}(\sim 1.8 \mathrm{eV})$ between the bonded and chemisorbed oxygen in alumina agreed with reported values in the literature (Renault et al., 2002)
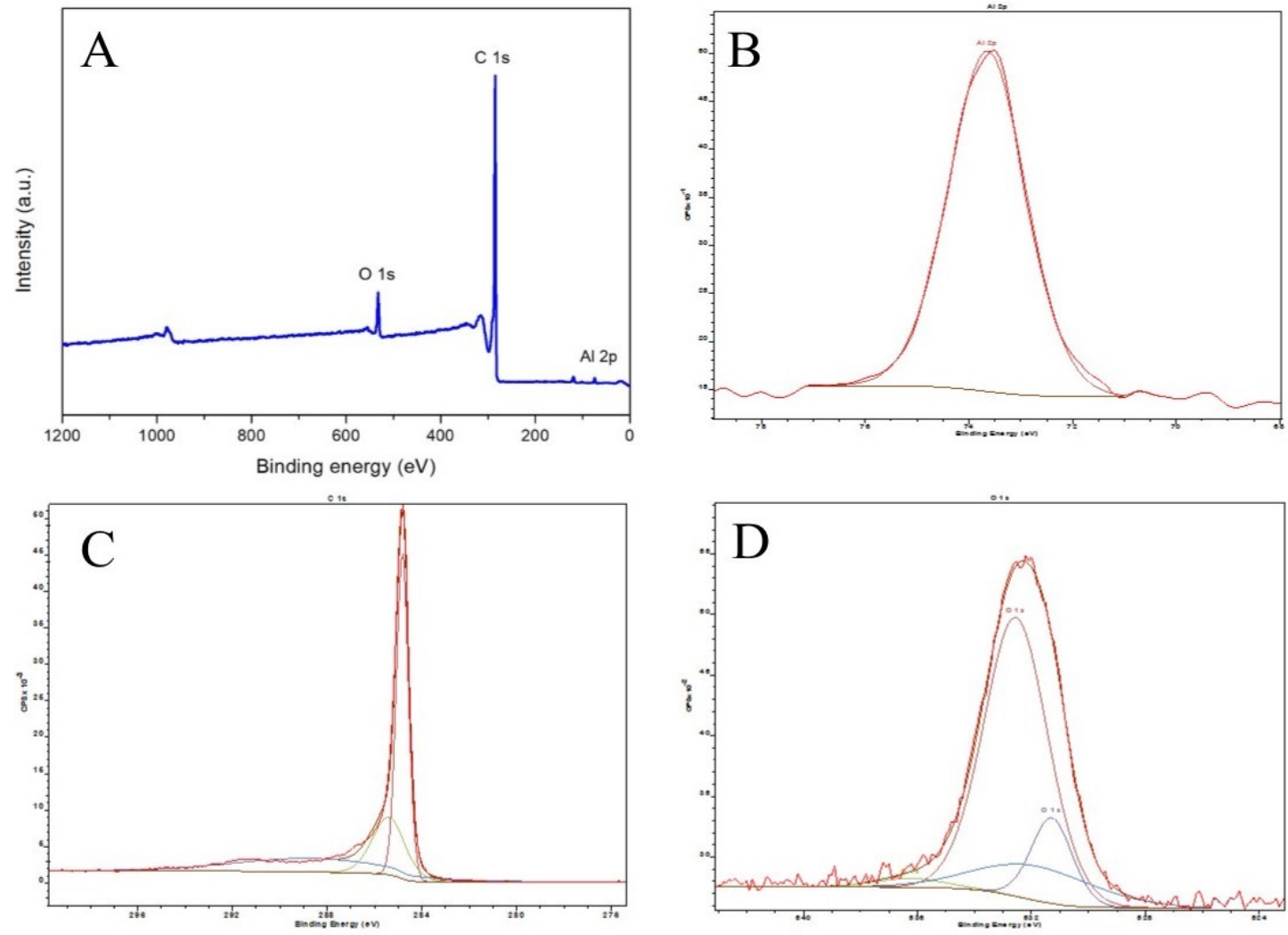

Figure 5.20: Full XPS spectra (A), Al 2p (B), C 1s (C) and O 1s (D) peak deconvolution of $\mathrm{Al}_{2} \mathrm{O}_{3} \mathrm{CNTs}$.

The full XPS survey spectra of Ag decorated MWCNTs samples are shown in (Figure 5.21A - Figure 5.21C). A clear distinction can be seen regarding the intensity of Ag peak due to the variation in the silver loading percent on the nanotubes. The 
Chapter Five - Fabrication and characterization of versatile metal/metal oxide carbon based hybrid nanostructures.

double peak (368.4 eV and $374.5 \mathrm{eV}$ ) in the Ag-CNTs, 10HNS and 20HNS XPS spectrum (Figure 5.21D) corresponds to $\mathrm{Ag} 3 \mathrm{~d}_{5 / 2}$ and $\mathrm{Ag} 3 \mathrm{~d}_{3 / 2}$ electronic states related to $\mathrm{Ag}^{0}$ (Rehan et al., 2015), where $\mathrm{Ag} 3 \mathrm{~d}$ region has well-splitting spin-orbit components $\left(\Delta_{\text {metal }}=6.0 \mathrm{eV}\right)$ (Figure 5.21D). These results are in agreement with that of metallic silver (Zhao et al., 2017). Figure 5.21E showcases the deconvolution of C1s spectra into major peaks from $\mathrm{sp}^{2}$ carbon at $\mathrm{BE}=284.8 \mathrm{eV}$ and two minor signals from $\mathrm{C}-\mathrm{O}(285.8 \mathrm{eV})$ and $\mathrm{C}=\mathrm{O}(288.8 \mathrm{eV})$. While for the $\mathrm{O}$ 1s spectra (Figure 5.21F), two signals were detected at $\mathrm{BE}=531.7 \mathrm{eV}$ and $\mathrm{BE}=533.3 \mathrm{eV}$, that refers to $\mathrm{O}=\mathrm{C}$ and $\mathrm{O}-\mathrm{C}$, respectively.
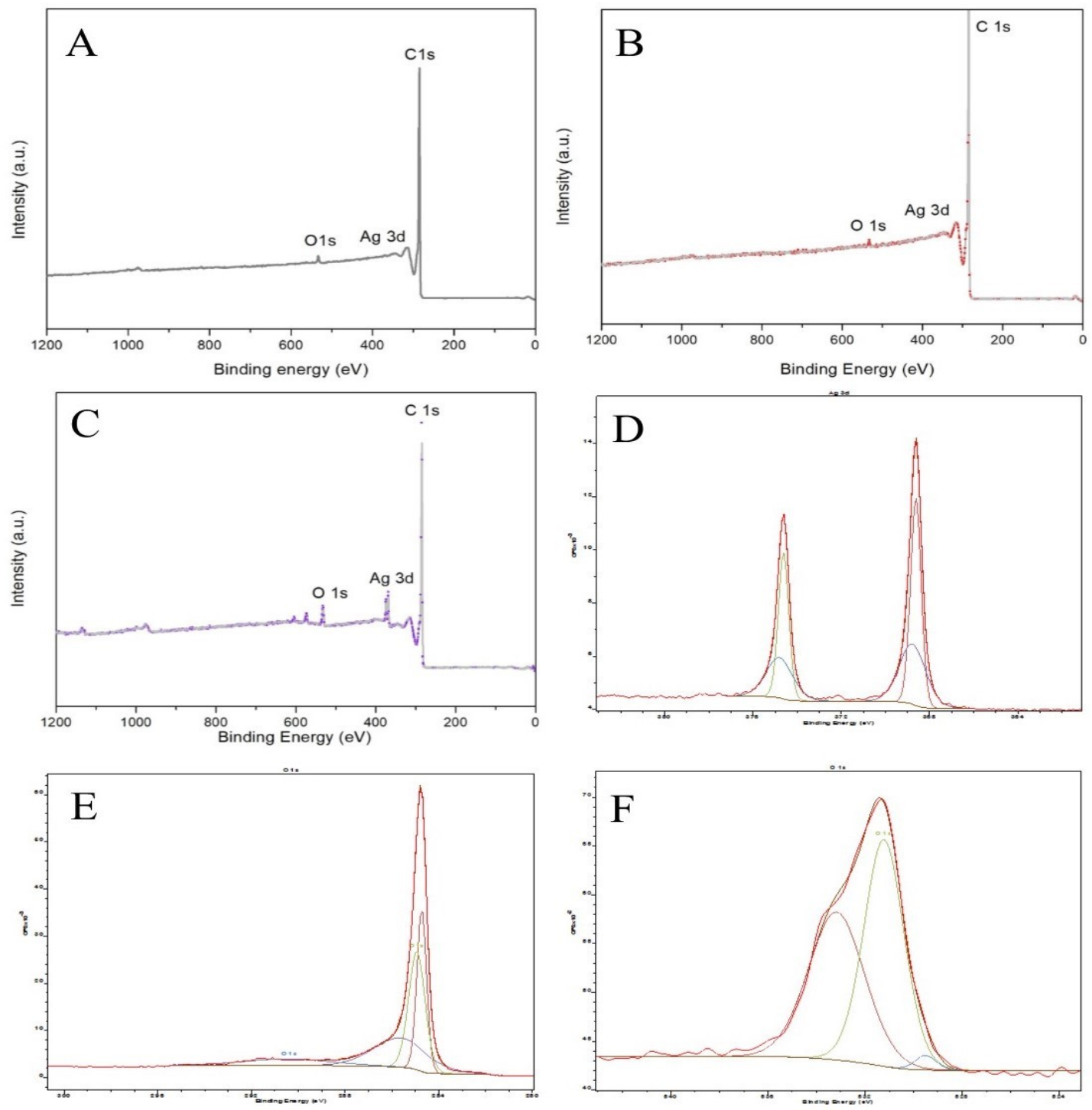

Figure 5.21: Full XPS spectra of AgCNTs (A), 10HNS (B), 20HNS (C), Ag 3d (D), C $1 \mathrm{~s}(\mathrm{E})$ and $\mathrm{O} 1 \mathrm{~s}(\mathrm{~F})$ peak deconvolution. 
Chapter Five - Fabrication and characterization of versatile metal/metal oxide carbon based hybrid nanostructures.

\subsection{Conclusions}

This part of the thesis has introduced a novel, facile and scalable routes for the design and fabrication of two groups of hybrid nanostructures for nanocomposite membrane applications. In the first group, HNS comprises two loading levels (10 and 20\%) of Ag on the surface of MWCNTs (10HNS and 20HNS). The HNS were fabricated via the wet chemistry method then followed by microwave irradiation. While four metal/metal oxides $\left(\mathrm{Fe}_{2} \mathrm{O}_{3}, \mathrm{TiO}_{2}, \mathrm{Al}_{2} \mathrm{O}_{3}\right.$, and $\left.\mathrm{Ag}\right)$ were deposited on the surface of nanotubes via same wet chemistry technique, and followed by calcination at a high temperature in a tube furnace. Prior to fabrication, the raw nanotubes were carboxylated and purified by concentrated $\mathrm{HNO}_{3}$. The successful fabrication process of all HNS was assessed comprehensively. The presence of $-\mathrm{COOH}$ groups was confirmed by FTIR while zeta potential measurements were carried to quantify the degree of functionalization for MWCNTs and influence of the later deposition of M/MO on the zeta potentials values. Microscopic images showcased no apparent damage to the nanotubes occurred after acid treatment. X-ray diffraction analysis evidenced that metal salts were presented in the metallic form on the surface of nanotubes. Structural changes prior and after nanotubes decoration were illustrated by Raman spectra. Impact of acid treatment and $\mathrm{M} / \mathrm{MO}$ deposition on the thermal properties of nanotubes were assessed by thermogravimetric analysis. Finally, the oxidation states of M/MO were confirmed by XPS. 


\section{Chapter Six}

\section{Fabrication of antibacterial nanocomposite membranes enabled by hybrid nanostructure modulated by microwave irradiation}

\subsection{Introduction}

Biological fouling is considered as the most inherently complex form of fouling. It is defined by microbial cell adhesion and subsequent colonisation at the membrane surface forming a microbial biofilm. Commonly, microbial colonisation of the membrane surface is initialised by irreversible adhesion of one type of bacteria or more, followed by the growth and multiplication of the surface-bound cells in the presence of feed nutrients (Flemming, 1997). Once the microbes attach at the surface and form a matrix of extracellular polymers, their removal will be extremely arduous even with the application of biocides (Subramani \& Hoek, 2008). Thus, biofouling represents the major concern for industries that exploit membrane technology including water, food, and pharmaceuticals. As an alternative to the disinfectant application, nanotechnology has impacted on the design and fabrication of nanocomposite membranes with the potential for creating self-cleaning and antimicrobial surfaces.

Within the wide range of commercially available nanoscale materials, silver nanoparticles (Ag-NPs) have gained special interest as unique antimicrobial additives in a broad range of applications. This is due to their unique antimicrobial properties 
Chapter Six - 6 Fabrication of antibacterial nanocomposite membranes enabled by hybrid nanostructure modulated by microwave irradiation.

alongside their electrical and optical characteristics (Ahamed et al., 2010; Iravani et al., 2014). Even though their antimicrobial mechanisms are still under debate and not comprehensively understood (Kim et al., 2007), their biocidal activity is well recognised as a highly desirable capability that they bestow on a membrane surface to resist biofouling and impart self-cleaning characteristics. On this basis, a large amount of work has been devoted for developing such nanocomposites membranes (Liu et al., 2017; Mollahosseini et al., 2012). As an important consideration when developing nanocomposite membranes for the control of biofouling, it is crucial to sustain antimicrobial activity and avoid the rapid depletion of nanosilver (nAg) that could diminish the antibacterial activity of $\mathrm{nAg}$, and increase environmental issues and cost constraints. Attempts have been stepped forward to ameliorate the interactions between these metallic nanoparticles and host polymeric chain to overcome these limitations. One of the most pragmatic approaches used for this target was through revising the surface characteristics of Ag-NPs via chemical treatment (Mauter et al., 2011). Various functional groups for modification/functionalization are available to increase their stability in the host polymer, e.g. phosphoric acid, carboxylic acid, dopamine and silane coupling agents (Dias et al., 2011).

Currently, intensive research are focusing on integration of one-dimensional nanoscale materials (1D-NMs) or two dimensional (2D-NMs) with zero-dimensional (0D-NPs) into one hybrid nanostructure (HNS), which found their way in countless applications such as; fuel cells, photocatalysis, electrocatalysis, solar cells, sensors, supercapacitors, batteries and hydrogen storage applications (Baughman et al., 2002). Applying these HNS could potentially exhibit not only the unique characteristics of $1 \mathrm{D} / 2 \mathrm{D}$ and $0 \mathrm{D}$ at the nanoscale level but also may possess novel chemical and physical properties as a result of the synergic effects of both nanomaterials that might not be obtainable to their individual component alone (Kang et al., 2008b). Several HNS targeting water and wastewater treatment have been reported in the recent few years (Chen et al., 2012; Gunawan et al., 2011; Roy et al., 2015; Wang et al., 2008; Welderfael et al., 2016; Xu et al., 2016a). Parallel to this steadily evolution in antimicrobial nanostructures and applications. Functionalization of polymeric membrane aiming for a specific application has been evinced with these tailored nanostructures. For antibacterial nanocomposite membranes, Xu et al. blended AgNPs decorated $\mathrm{Cu}_{2} \mathrm{O}$ nanowires (NWs) with PSF via phase inversion. To facilitate the 
Chapter Six - 6 Fabrication of antibacterial nanocomposite membranes enabled by hybrid nanostructure modulated by microwave irradiation.

deposition of AgNPs on the surface of $\mathrm{Cu}_{2} \mathrm{O}$, L-dopa has been grafted onto NWs surface by in situ polymerisation to create a zwitterionic surface. The nanocomposite membranes held remarkable antibacterial activity against E. coli and S. aureus with controlled release of $\mathrm{Ag}^{+}$(Xu et al., 2016b). Recently, Faria et al. conducted a research to functionalize TFC membranes with GO-Ag. Results referred to a promising inactivation rate against attached $P$. aeruginosa (up to 80\%) without sacrificing the intrinsic transport properties of the modified membranes (Faria et al., 2017). A number of HNS employed in fabrication multifunctional nanocomposite membranes have been reported in the literature for wastewater treatment and desalination applications (Chen et al., 2012; Gunawan et al., 2011; Roy et al., 2015; Xu et al., 2016a). Another interesting 1D nanoscale carrier is carbon nanotubes (CNTs). Unsurprisingly, the assembly of CNTs along with the antibacterial Ag-NPs into one synergic HNS enticed tremendous applications, especially those targeting antimicrobial and self-cleaning surfaces. Utilization of CNTs as a carrier could hinder the aggregation and weak stability of unmodified AgNPs and ultimately broadens their potential applications as unique antibacterial additives in nanocomposite membrane applications.

There are a number of methods that have been developed for anchoring metallic NPs on the surface of CNTs, which include chemical reduction, pulsed laser ablation, microwave treatment, solar radiation, electrochemical deposition and in situ deposition on modified CNTs. Among these techniques, microwave treatment with its beneficial characteristics of energy rapid heating and decreased sintering temperatures has broad application. Microwave energy can be used in chemical synthesis, processing of new materials, and in purification methods of carbon nanotubes (Gomez et al., 2012; Vázquez \& Prato, 2009). CNTs suffer a rapid temperature increase under microwave irradiation (Vázquez \& Prato, 2009). By using the rapid and local Joule heating of carbon nanomaterials under microwaves, metallic NPs can be created on the surface of the nanotubes, where the localized heat created on the surface of the carbon nanomaterials can be used to decompose different metallic precursors resulting in the immediate creation of metal and metal oxide particles (Lin et al., 2011).

To the best of the authors' knowledge, no one, so far, has employed microwave irradiation in the preparation of HNS for nanocomposite membranes fabrication. Moreover, to assess and compare the influence of varying Ag loading wt.\% to CNTs on the morphological and antibacterial characteristics of nanocomposite membranes 
Chapter Six - 6 Fabrication of antibacterial nanocomposite membranes enabled by hybrid nanostructure modulated by microwave irradiation.

\subsection{Synthesis of hybrid nanostructured materials}

For more description details on purification and decoration of nanotubes with silver NPs, please refer to Section 5.2.

\subsection{Membrane fabrication with HNS}

All nanocomposite membranes were prepared via the classical phase inversion technique reported in section 3.3.1 (Chapter 3). Two groups of modified membranes, using 10HNS and 20HNS were prepared. For each group, the amount of HNS added to the polymer (PES) was varied, see compositions in Table 6.1, to assess their influence on the overall performance of the nanocomposite membranes.

In the first instance, $2 \mathrm{wt} . \%$. of PVP K30 (as pore former) was dissolved in NMP for an hour at $50{ }^{\circ} \mathrm{C}$, the solution was then cooled to room temperature before addition of HNS at the desired loading weight. Thereafter, the solution was sonicated for $30 \mathrm{~min}$ using a horn sonicator. The RB flask was placed back on the heating plate and PES flakes (16 wt.\%) were gradually added to the precursor and vigorously mixed overnight. Degassing of the casting solution was carried out using a desiccator under vacuum condition for $1 \mathrm{~h}$. For solution casting, about $10 \mathrm{ml}$ of casting solution was poured onto a glass substrate and cast with an automated casting knife (RK film applicator) at a regular shear rate $\left(225 \mathrm{~s}^{-1}\right)$ with $200 \mathrm{~mm}$ clearance gap at ambient temperature. The resulting thin film was placed in a DI water bath at $20^{\circ} \mathrm{C}$ for precipitation. Within less than two minutes, the membrane detached from the glass plate indicating that the phase inversion was complete the membrane remained in the water bath for another $30 \mathrm{~min}$. Finally, the membrane was washed with DI water and stored under water (DI) in a sealed container at $4{ }^{\circ} \mathrm{C}$, to be used in characterisation. All membranes were cast at room temperature and relative humidity $(\mathrm{RH} \% 45 \pm 5)$. Prior to testing, the membranes were inspected under light to make sure there were no pinholes, wrinkles or any defect that could make a variation in their performance. 
Chapter Six - 6 Fabrication of antibacterial nanocomposite membranes enabled by hybrid nanostructure modulated by microwave irradiation.

Table 6.1: Compositions of nanocomposite membranes using two concentrations of silver to nanotubes.

\begin{tabular}{llllll}
$\begin{array}{l}\text { Nanocomposite } \\
\text { membrane ID }\end{array}$ & \multicolumn{6}{l}{ Compositions } & & \\
& PES \% & PVP \% & NMP \% & 10 HNS \% & 20HNS \% \\
\hline Control & 16 & 2 & 82 & 0 & 0 \\
\hline $0.05-10 \mathrm{M}$ & 16 & 2 & 82 & 0.05 & 0 \\
$0.1-10 \mathrm{M}$ & 16 & 2 & 82 & 0.1 & 0 \\
$0.3-10 \mathrm{M}$ & 16 & 2 & 82 & 0.3 & 0 \\
$0.9-10 \mathrm{M}$ & 16 & 2 & 82 & 0.9 & 0 \\
\hline $0.05-20 \mathrm{M}$ & 16 & 2 & 82 & 0 & 0.05 \\
$0.1-20 \mathrm{M}$ & 16 & 2 & 82 & 0 & 0.1 \\
$0.3-20 \mathrm{M}$ & 16 & 2 & 82 & 0 & 0.3 \\
$0.9-20 \mathrm{M}$ & 16 & 2 & 82 & 0 & 0.9 \\
\hline
\end{tabular}

\subsection{Nanocomposite membranes}

To establish a deeper insight into the amelioration of membrane materials when they are fabricated by doping with HNS to produce nanocomposite membranes, an extensive characterisation of the membrane parameters that influence membrane performance was undertaken. Thus, nanocomposite membranes were characterised in terms of PWF, pore size and pore size distribution, hydrophilicity, surface zeta potential measurements and surface morphology. Moreover, the bacteriostatic activity of these nanocomposites was also tested to confirm the efficacy of the active materials after membrane fabrication and to identify the influence of loading wt.\% of HNS and Ag loading level to MWCNTs on the prepared nanocomposites. It should be noted that increasing the concentration of both $10 \mathrm{HNS}$ or $20 \mathrm{HNS}$ in the casting solution resulted in darker nanocomposites; the undersides of the membranes were significantly lighter in colour than the top surface for all nanocomposites, (Figure 6.1). This was due to the migration and concentration of the HNS at the top surface during the phase inversion process. Many factors can be adjusted to induce this migration of HNS to top surface of nanocomposites, including the use of smaller nanotubes dimensions (length and diameter), and playing with the PI process parameters aiming to induce rapid demixing. 
Chapter Six - 6 Fabrication of antibacterial nanocomposite membranes enabled by hybrid nanostructure modulated by microwave irradiation.

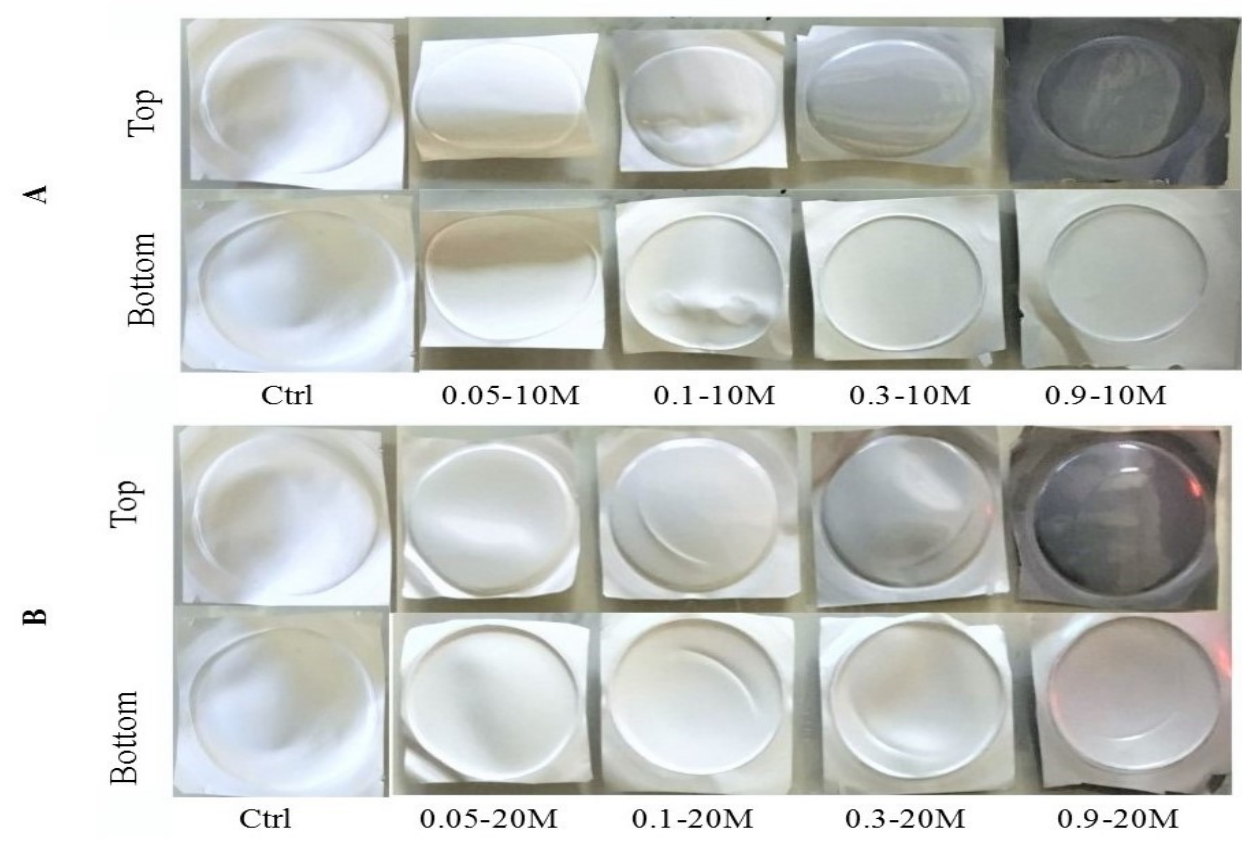

Figure 6.1: The top and bottom surface of (A) nanocomposites prepared with 10HNS and (B) nanocomposites prepared with 20HNS.

\subsubsection{Cross section morphology of nanocomposites}

A cross-sectional study was conducted by SEM to qualitatively assess the cross-section morphology of the nanocomposite membranes since the structure has a significant influence on membranes transport mechanisms. All cross-sectional images obtained from bare and nanocomposite membranes demonstrate a typical asymmetric structure, (Figure 6.2). A clear dense active and porous supporting layer can be observed for all the membranes. Control PES membrane had a well-developed skin layer supported by wide finger-like macro-pores at the bottom of the membrane, (Figure 6.2A). This structure was formed due to the low polymer dope solution used to prepare the membrane. The structure is favoured as at low viscous casting solutions the nonsolvent exchange rate into the polymer lean phase exceeds the outward solvent diffusion rate (Sofiah et al., 2010). Incorporating a small amount of the HNS into the PES matrix (up to $0.1 \%$ ) promoted the formation of big macrovoids in the sublayer, (Figure 6.2B and Figure 6.2C). While the further increase in HNS content suppressed the formation of these macrovoids and led to narrower micro-pores with a finger-like shape oriented from the top to the bottom layer of the nanocomposites, this is a different shape to the micro-pores of the PES membrane, as seen in (Figure 6.2D and Figure 6.2E). This was due to the enhanced viscosity of the casting solution that hinders the solvent-nonsolvent 
Chapter Six - 6 Fabrication of antibacterial nanocomposite membranes enabled by hybrid nanostructure modulated by microwave irradiation.

exchange rate at the interface between the surface and the nonsolvent, producing a slightly denser skin layer and less porous membranes (Rahimpour et al., 2012). On the other hand, the 0.9-20 M and 0.9-10 $\mathrm{M}$ membranes had similar cross-sectional morphology, indicating no significant variation in their permeation characteristics would result from variation of the silver level in the HNS (Figure 6.2F).
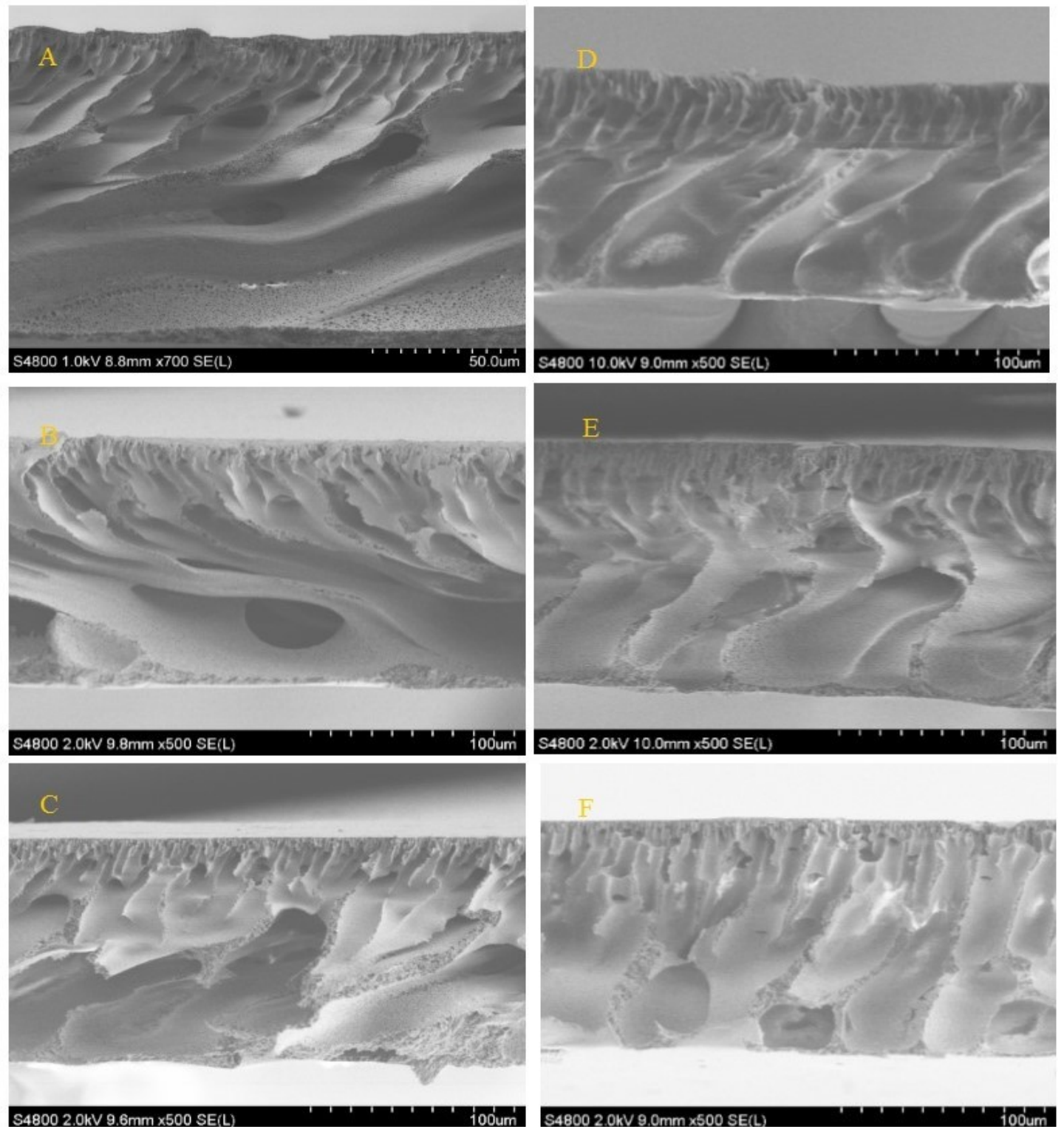

Figure 6.2: SEM cross section images for (A) Nascent PES membrane, (B) 0.05-10 $\mathrm{M},(\mathrm{C})$ 0.1-10 M, (D) 0.3-10 M, (E) 0.9-10 M, and (F) 0.9-20 M.

\subsubsection{Static contact angle and hydrophilicity measurements}

Contact angle measurement is a well-known technique for characterising surface hydrophilicity/hydrophobicity, which informs the analysis of membrane performance. A more hydrophilic membrane surface will result in a lower contact angle (Gilron et 
Chapter Six - 6 Fabrication of antibacterial nanocomposite membranes enabled by hybrid nanostructure modulated by microwave irradiation.

al., 2001). However, measurement of contact angle to express the hydrophilicity of a membrane material is not necessarily conclusive, since several factors may impact on the hydrophilicity values such as porosity, roughness, pore size and pore size distribution (Rana \& Matsuura, 2010). As shown in (Figure 6.3), the surface hydrophilicity/hydrophobicity of all nanocomposite membranes was lower compared to the nascent PES membrane. A noticeable gradual decrease in contact angle value was associated with each incremental increase in the concentration of HNS in the nanocomposites. This amelioration in hydrophilicity is believed to be induced by the spontaneous migration and concentration of the well-dispersed hydrophilic HNS close to the top surface/water interface to reduce the interfacial energy during the phase inversion. This phenomenon imparted more hydrophilicity to the hybrid nanocomposites (Balasubramanian \& Burghard, 2005). These results are in contradiction with those conducted previously and revealed irregular positioning of nanotubes within the structure of nanocomposites, at higher loading than $0.4 \mathrm{wt} . \%$ (Khalid et al., 2015). Both sets of nanocomposite membranes, supplemented with $10 \mathrm{HNS}$ and 20HNS, had a comparable contact angle value (slightly higher hydrophilicity for 10HNS nanocomposites) when an identical concentration of the HNS was used. This suggests that the Ag loading level to the MWCNTs had little influence on the contact angle measurement of the nanocomposite membrane.

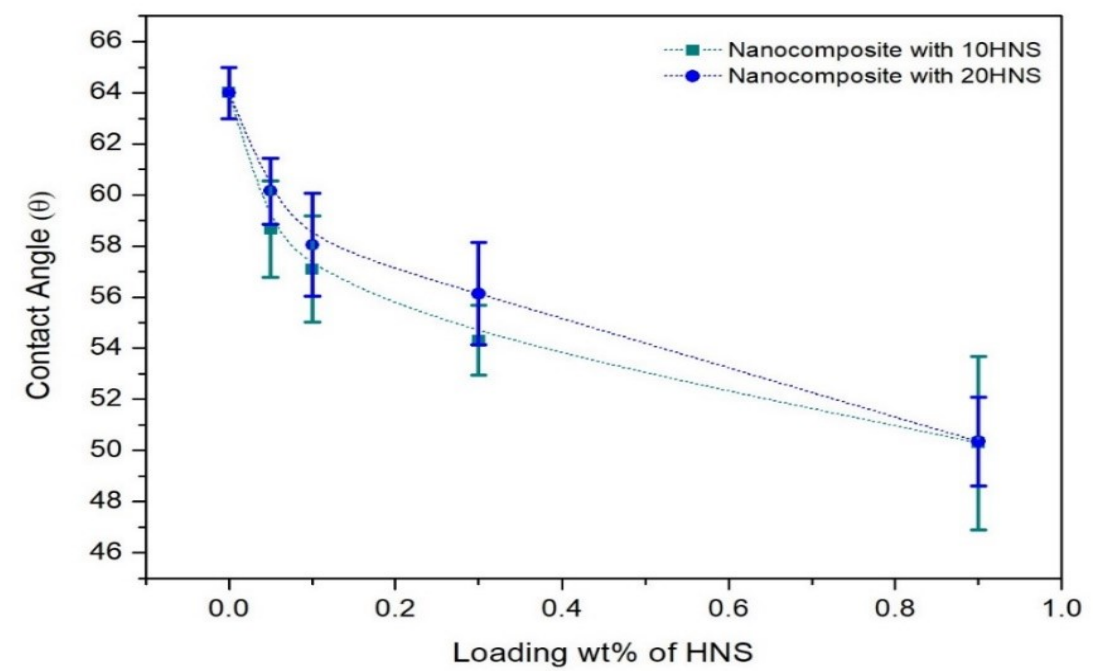

Figure 6.3: Contact angle measurements of nanocomposite membranes prepared with different loading weights of $10 \mathrm{HNS}$ and 20HNS. 
Chapter Six - 6 Fabrication of antibacterial nanocomposite membranes enabled by hybrid nanostructure modulated by microwave irradiation.

\subsubsection{Mean pore size and pore size distribution determination}

A solute transport model, as a standardised method, has been applied to determine the nanocomposite membranes geometric mean pore size $\left(\mu_{\mathrm{p}}\right)$, geometric standard deviation $\left(\sigma_{\mathrm{p}}\right)$ around the mean pore size, and their distribution characteristics. The $\mu_{\mathrm{p}}$ and $\sigma_{\mathrm{p}}$ of the unmodified (control) and nanocomposites membranes are summarised in Table 6.2. Addition of $10 \mathrm{HNS}$ to the control membrane did not dramatically change the surface pore size characteristics, at all loading compositions. This indicates a very high degree of 10HNS dispersion inside the PES matrix. Indeed, negligible variation in the nanocomposites selectivity was obtained. Mean pore diameter of membranes were found to slightly increase from $10.93 \mathrm{~nm}$ to $11.69 \mathrm{~nm}$ with only $0.05 \% 10 \mathrm{HNS}$. Further increase in the $10 \mathrm{HNS}$ composition, to 0.1 and $0.3 \%$, led to a gradual decrease in the pore size. This decrease reached its minimum value at $0.9 \%$ with a pore diameter of $10.4 \mathrm{~nm}$. It was expected that a very high loading (0.9\%) would induce pore size narrowing due to the higher casting solution viscosity, which resulted from adding this amount of HNS. Geometric standard deviation values were comparable and reasonable for all UF membranes. Values of $\sigma_{\mathrm{p}}$ associated with their corresponding $\mu_{\mathrm{p}}$ values ranged from 1.27 to 1.44 . This indicates that all unmodified and nanocomposites were similar in their microstructure. On the other hand, incorporation of 20HNS into the polymeric matrix showed slightly higher $\mu_{\mathrm{p}}$ and $\sigma_{\mathrm{p}}$ values to those obtained from nanocomposites prepared using 10HNS.

Table 6.2: Geometric mean pore size and standard deviation of nanocomposite membranes.

\begin{tabular}{lll} 
Membrane ID & $\begin{array}{l}\text { Mean pore diameter } \\
\left(\boldsymbol{\mu}_{\mathbf{p}}\right)(\mathbf{n m})\end{array}$ & $\begin{array}{l}\text { Geometric standard } \\
\text { Deviation }\left(\boldsymbol{\sigma}_{\mathbf{p}}\right)\end{array}$ \\
\hline Control & 10.93 & 1.37 \\
\hline $0.05-10 \mathrm{M}$ & 11.69 & 1.28 \\
$0.1-10 \mathrm{M}$ & 11.15 & 1.34 \\
$0.3-10 \mathrm{M}$ & 11.1 & 1.38 \\
$0.9-10 \mathrm{M}$ & 10.4 & 1.40 \\
\hline $0.05-20 \mathrm{M}$ & 12.9 & 1.27 \\
$0.1-20 \mathrm{M}$ & 12.3 & 1.29 \\
$0.3-20 \mathrm{M}$ & 10.65 & 1.44 \\
$0.9-20 \mathrm{M}$ & 10.57 & 1.40 \\
\hline
\end{tabular}


Chapter Six - 6 Fabrication of antibacterial nanocomposite membranes enabled by hybrid nanostructure modulated by microwave irradiation.

The cumulative pore size distributions of the nanocomposites are presented in (Figure 6.3 and Figure 6.4). This provides further evidence that there was negligible difference in pore size distributions for all PES membrane. HNS addition, up to $0.3 \%$, caused a slight shift to the right for the pore size curves. At $0.3 \% \mathrm{HNS}$, the curve was almost identical to the control PES membrane, which exhibited similar mean pore size and standard deviation. While only $0.9-10 \mathrm{HNS}$ nanocomposite curve shifted to the left. This behaviour of the pore size distribution curves suggests no appreciable agglomeration of the HNS within the polymer matrix occurred even at high HNS content.

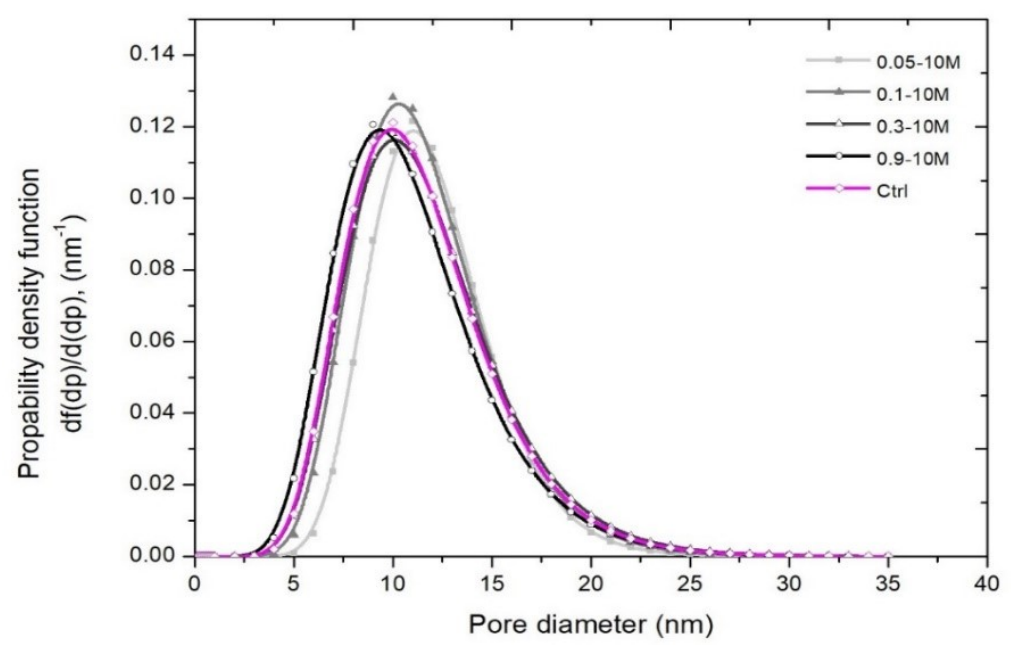

Table 6.3: Pore size cumulative curves of nanocomposite membranes for 10HNS.

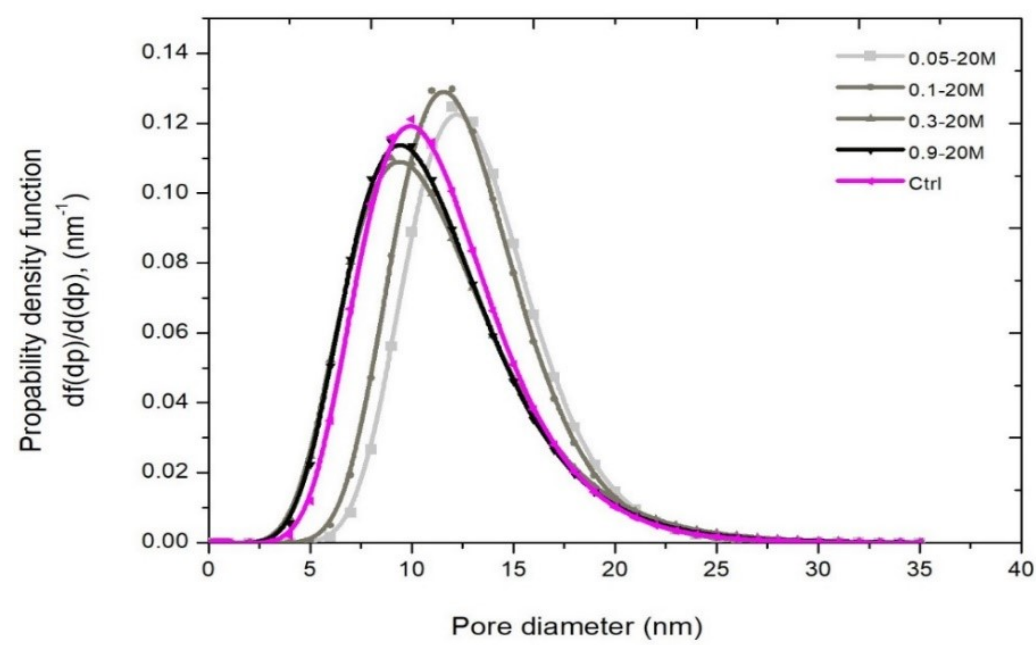

Figure 6.4: Pore size cumulative curves of nanocomposite membranes for $20 \mathrm{HNS}$. 
Chapter Six - 6 Fabrication of antibacterial nanocomposite membranes enabled by hybrid nanostructure modulated by microwave irradiation.

\subsubsection{Surface zeta potential of nanocomposite membranes}

Surface zeta potential $(\zeta)$ of the membranes was characterised using a streaming potential technique. Zeta potential measurements are indispensable for fundamental insights into the separation mechanisms, membrane ageing, membrane fouling and cleaning. All modified and unmodified membranes were found to be negatively charged from $\mathrm{pH} 2.5$ to $\mathrm{pH} 10.5$, no isoelectric points could be identified, (Figure 6.5). Results obtained for the nascent PES membrane were in agreement with previous research (Liu et al., 2015). Incorporating the HNS within the polymeric matrix was seen to have a palpable influence on the membrane, depending on the HNS concentration added. To the best of the authors' knowledge, there has been no research examining the influence of loading weight, for any metal-carbon based HNS to a polymeric membrane, on surface zeta potential. Nanocomposite membranes with the lowest concentration of HNS exhibited the highest negative zeta potential values compared to others. Increasing the HNS loading to the PES not only lowered the negative zeta potential value of nanocomposites but also narrowed the range of zeta potential measurements over the entire $\mathrm{pH}$ range studied. This upward trend was attributed to the positively charged AgNPs in the HNS. Higher amounts of HNS in the nanocomposite membrane gave rise to higher amounts of $\mathrm{Ag}^{+}$to outweigh their counterpart negatively charged MWCNTs.

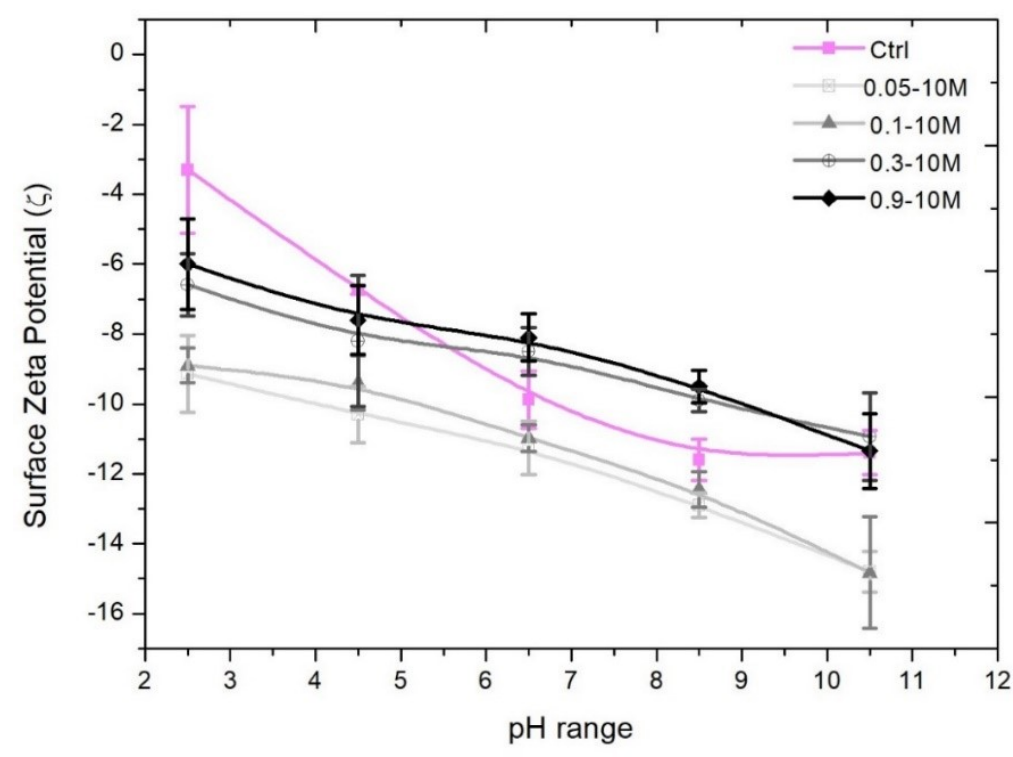

Figure 6.5: Surface Zeta potential of nascent and nanocomposites membranes at different loading weight of $10 \mathrm{HNS}$. 
Chapter Six - 6 Fabrication of antibacterial nanocomposite membranes enabled by hybrid nanostructure modulated by microwave irradiation.

\subsubsection{Pure water flux}

The Pure water flux (PWF) was computed using a crossflow filtration rig and having an active membrane area of $12.6 \mathrm{~cm} 2$. After compacting the nanocomposite coupons at a transmission pressure of 5 bar for approximately $30 \mathrm{~min}$, the pressure was reduced to 4 bars and the permeate flux was recorded automatically, as described in section 3.3.4. PWF of the nanocomposites incorporating10HNS and 20HNS are presented in (Figure 6.6). All nanocomposite membranes had improved PWF behaviour as HNS content increased. Compared with unmodified PES membrane, the 10HNS concentration in the nanocomposite, from 0 to $0.05,0.1,0.3$ and $0.9 \%$, raised the PWF from $554\left(\mathrm{~L} \mathrm{~m}^{-2} \cdot \mathrm{h}^{-1}\right)$ to $693,722,746$ and $556\left(\mathrm{~L}_{\mathrm{m}} \mathrm{m}^{-2} \cdot \mathrm{h}^{-1}\right)$, respectively. Interestingly, even though the 0.9 10HNS nanocomposite returned to a lower PWF, it was still comparable to that of unmodified PES membrane. Herein, the PWF data can explain the inter-correlation between the permeation characteristics of membranes and other characteristics such as hydrophilicity and solution casting viscosity. For instance, hydrophilicity data obtained have been agreed with PWF measurements for all nanocomposites, except 0.9-10HNS which had the lowest contact angle but lower flux than other nanocomposites, as illustrated in (Figure 6.3). The reduction in the flux may be attributed to the higher viscosity at $0.9 \%$ HNS concentration level that resulted in a slightly lower mean pore size and less porous structure, regardless of the lowest contact angle reported for this nanocomposite. This has been illustrated in the cross-section images earlier (section 6.4.1). To emphasise the role of hydrophilicity on the PWF of nanocomposite membranes, the 0.3-10HNS nanocomposite membranes had less porous structure than unmodified PES, a similar $\mu_{\mathrm{p}}$, but their PWF were significantly different. So far, the hydrophilicity effect can be considered as the dominant factor when the viscosity role is equimolar or low enough to discard it. On the other hand, nanocomposite membranes prepared using 20HNS displayed a similar trend but with higher PWF than membranes prepared with 10HNS. This may be due to the higher pore size obtained since the hydrophilicity of different membrane groups were almost identical. 
Chapter Six - 6 Fabrication of antibacterial nanocomposite membranes enabled by hybrid nanostructure modulated by microwave irradiation.

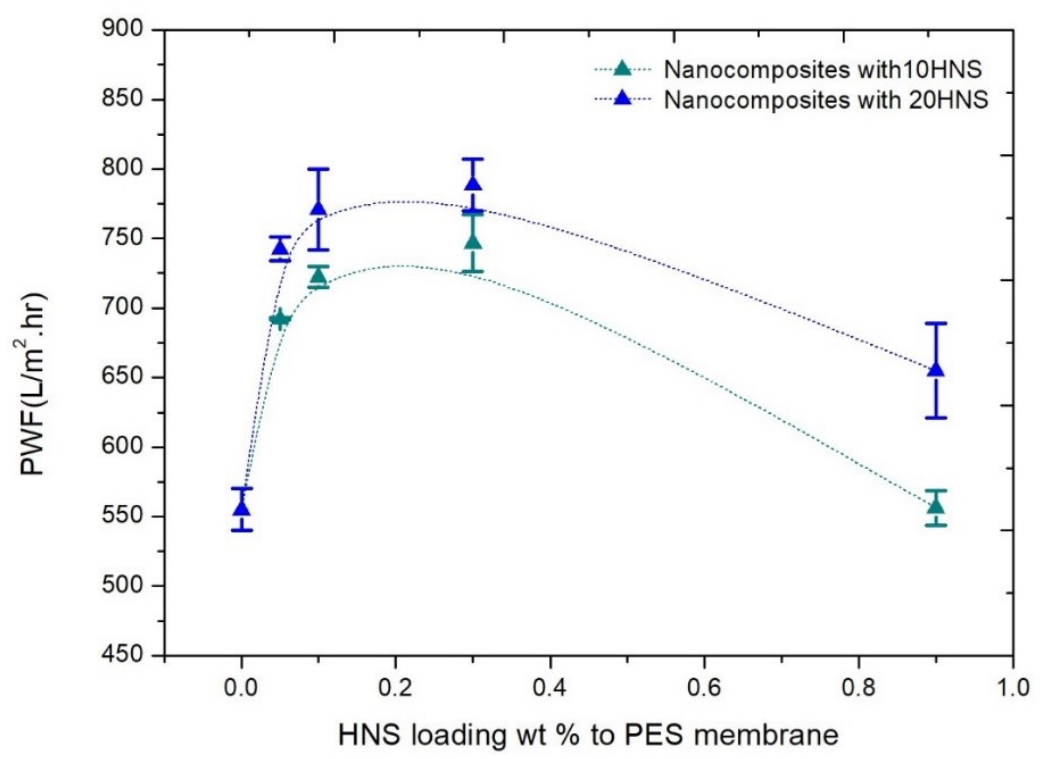

Figure 6.6: Pure water flux behaviour of nanocomposite membranes prepared with $10 \mathrm{HNS}$ and $20 \mathrm{HNS}$ at different loading wt.\%.

\subsection{Potential bacteriostatic activities of HNS and nanocomposites}

\subsubsection{Bacteriostatic of HNS}

The potential antibacterial activity of HNS was demonstrated using Zone of Inhibition (ZOI) test. Two species of bacteria were used in this test; Escherichia coli (ACIB 8277) as a Gram-negative representative species and Staphylococcus aureus (ATCC 6538P) as a Gram-positive representative species. A single colony of each species taken from a nutrient agar Petri plate culture was used as an inoculum and cultured in $5 \mathrm{ml}$ Mueller Hinton Broth (MHB) and incubated at $37{ }^{\circ} \mathrm{C}$ for $20 \mathrm{~h}$ under shaking (40 rpm). Appropriate culture suspensions were then diluted to achieve a standard concentration of 0.5 McFarland determined at 600ODs, using a microplate reader (Figure 3.19). In the meantime, nutrient agar medium was prepared, sterilised in an autoclave at $121^{\circ} \mathrm{C}$, then poured into sterile $90 \mathrm{~mm}$ plates and allowed to set at ambient temperature. Thereafter, $200 \mathrm{ml}$ of bacterial cultures was pipetted on the surface of the agar, spread using sterile cotton swap, and left for a 10 -min period for pre-incubation. Later, $3 \mathrm{mg}$ of each sample (f-MWCNTs, 10HNS, 20HNS) was placed on the surface of the agar 
Chapter Six - 6 Fabrication of antibacterial nanocomposite membranes enabled by hybrid nanostructure modulated by microwave irradiation.

and incubated at $37^{\circ} \mathrm{C}$ for $24 \mathrm{~h}$. Finally, the plates were inspected for any inhibition zone of growth and the diameter of the zone measured.

The variation in the zone of inhibition (ZOI) caused by samples of the nanoparticles can be observed in (Figure 6.7). Both 10HNS and 20HNS have manifested bacteriostatic capabilities against both E. coli and $S$. aureus. The ZOI is shown by the clear ring surrounding the sample, which resulted from the prevention of microbial growth. Meanwhile, there was no observable inhibition with the f-MWCNTs as shown in (Figure 6.7A). 10HNS had higher antibacterial activity against both species compared to that of the 20HNS sample. ZOI diameter was found to be $19.7 \mathrm{~mm}$ and $15.2 \mathrm{~mm}$ for 10HNS against E. coli and S. aureus, respectively (Figure 6.7B). While ZOI diameter decreased to $12.5 \mathrm{~mm}$ and $10.9 \mathrm{~mm}$, against E. coli and $S$. aureus respectively, when using $20 \mathrm{HNS}$ at the same inoculum concentration $\left(1.5 \times 10^{8} \mathrm{CFU} \mathrm{ml}^{-}\right.$ $\left.{ }^{1}\right)$, (Figure 6.7C). The higher inhibition of nanostructures towards E. coli as compared to that of S.aureus was thought to be due to the variance in cell wall structure, the difference in the thickness of peptidoglycan layer between Gram-negative and Grampositive bacteria. A thicker peptidoglycan layer of the cell wall is associated with the Gram-positive $S$. aureus $(20-80 \mathrm{~nm})$ compared to the Gram-negative E. coli $(7-8 \mathrm{~nm})$ (Madigan \& Martinko, 2005). The different activity of the silver ions against the different bacterial types may also be due to differences in the charge nature of the different cell walls of Gram-positive and Gram-negative bacteria; the electrostatic attraction between positively charged $\mathrm{Ag}$ and the negatively charged cell walls may be different (Kim et al., 2007), leading to different degrees of hindrance as the silver ions approach the microbial cells. 
Chapter Six - 6 Fabrication of antibacterial nanocomposite membranes enabled by hybrid nanostructure modulated by microwave irradiation.
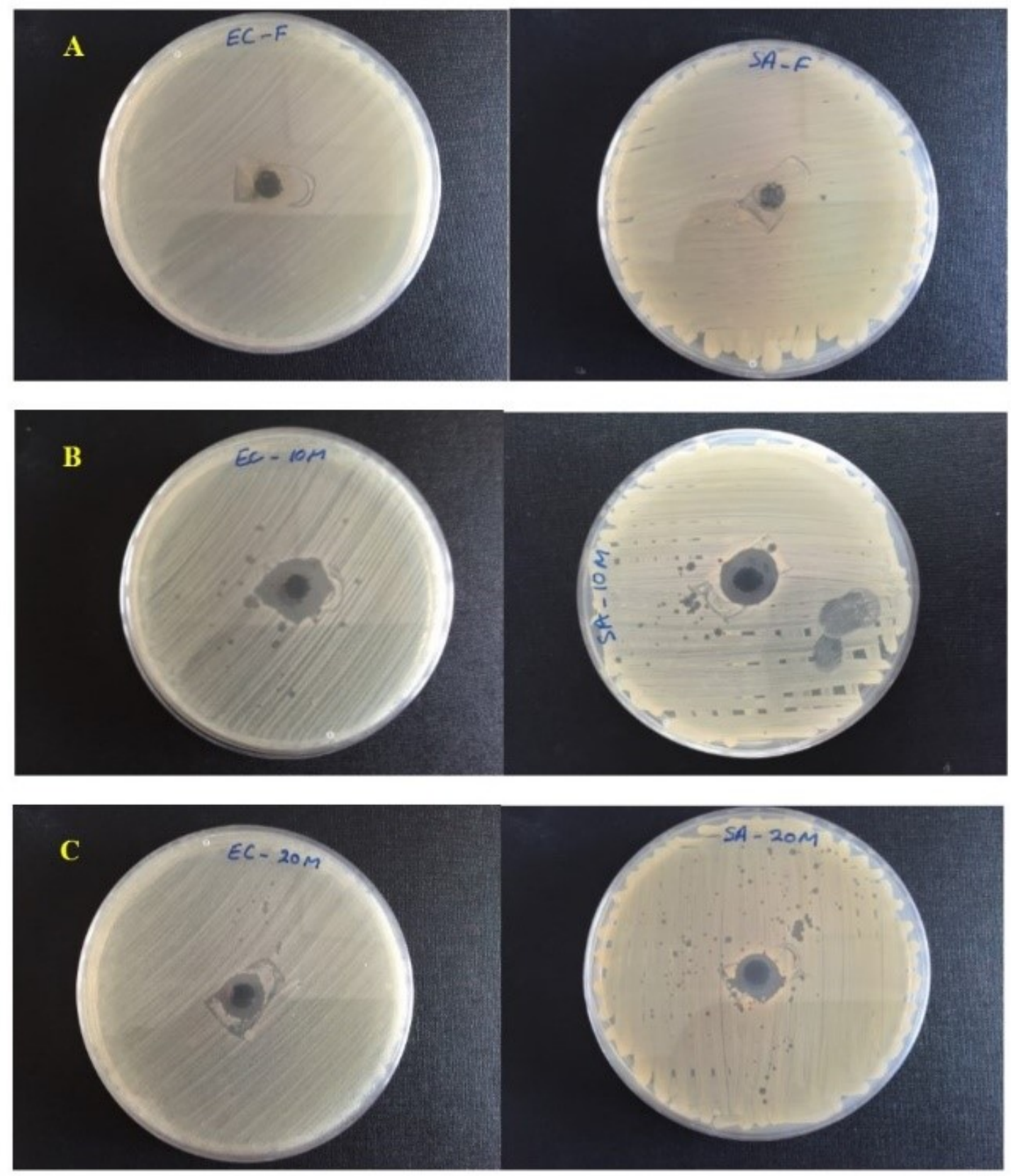

Figure 6.7: Inhibition zone against EC (Left) and SA (Right) for (A) f-MWCNTs, (B) $10 \mathrm{HNS}$ and (C) 20HNS.

\subsubsection{Bacteriostatic activities of nanocomposite membranes}

The potential antibacterial activities of nanocomposite membranes were determined using ISO 20743 test. Two representative microorganism samples used in ZOI tests, $E$. coli and $S$. aureus were applied for this purpose. A single colony of each strain, taken from an inoculum plate, was cultured in $5 \mathrm{ml} \mathrm{MHB}$ and incubated at $37^{\circ} \mathrm{C}$ for $20 \mathrm{~h}$ under shaking (40 rpm). Appropriate culture suspensions were then diluted to achieve a standard concentration of $\left(1.0 \times 10^{6} \mathrm{CFU} / \mathrm{ml}\right)$ determined at $600 \mathrm{OD}$. Meanwhile, each 
Chapter Six - 6 Fabrication of antibacterial nanocomposite membranes enabled by hybrid nanostructure modulated by microwave irradiation.

nanocomposite sample was cut into $1.5 \times 1.5 \mathrm{~cm}^{2}$ pieces and sterilised in $70 \% \mathrm{EtOH}$, rinsed with sterile DI water and allowed to dry at ambient temperature and sterile conditions. The membranes were then kept in sterile Falcon tubes and $50 \mu \mathrm{L}$ inoculum was spread over the sample surface using disposable loops and then incubated for 24-h period at $37^{\circ} \mathrm{C}$. As a standard step in the ISO test procedure, $5 \mathrm{ml}$ neutraliser was added to the samples aiming to stop any potential growth of species after this stage through creating an environment free of nutrients. The suspension was then vortexed to allow the bacteria to detach from the membrane surface. Thereafter, $1 \mathrm{ml}$ of the bacterial suspension was taken to make a serial dilution (up to 8 dilutions) using Phosphate Buffer Solution (PBS) at ratio 1/9 (suspension/diluent) for each dilution. 4-6 droplet $\left(20 \mu \mathrm{L}\right.$ each) were then cultured on nutrient agar plates prior to incubation at $37^{\circ} \mathrm{C}$ for 24-h. Finally, a colony plate counting method was used to count only the viable discrete colonies on the plate, an example is given in Figure 6.8. In this established procedure, valid counts should only consider a certain number of colonies for each drop; minimum 2 to a maximum of 20. Any count not within this range was attributed to invalid results. This method has been widely applied in the literature and considered as a reliable, fast and precise way of determining microbial cell viability at surfaces (Singh et al., 2012). To assess the antibacterial activity of the nanocomposites, the number of CFU/ml obtained after exposing the membranes to the bacterial cultures was determined and results were expressed in Log kill (Log of reduction factor calculated from dividing of $\mathrm{CFU} / \mathrm{ml}$ obtained from nanocomposites to that obtained from the control membrane). 
Chapter Six - 6 Fabrication of antibacterial nanocomposite membranes enabled by hybrid nanostructure modulated by microwave irradiation.
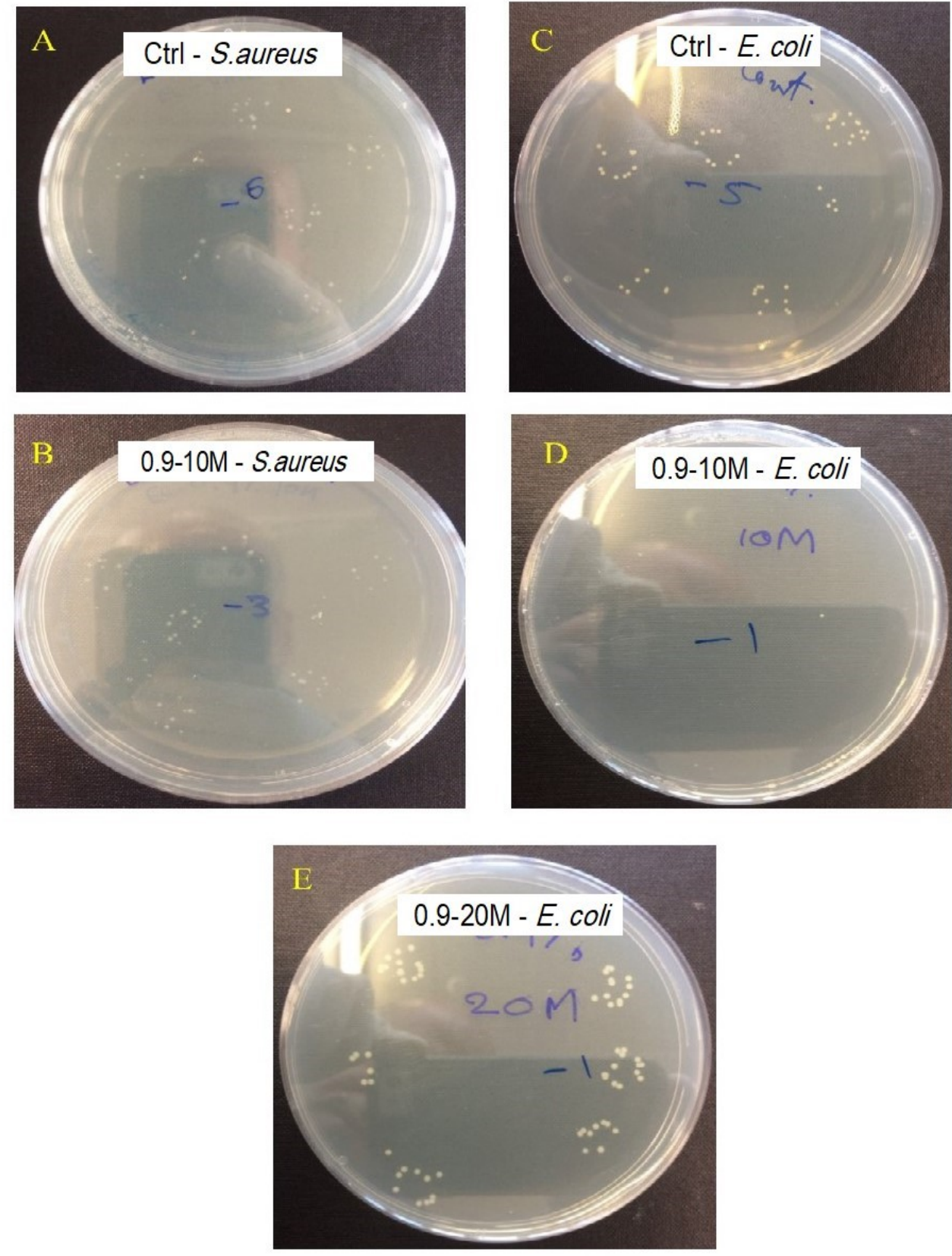

Figure 6.8: Example illustrates the growing colonies of viable $S$. aureus and E. coli colonies on nutrient agar medium for $(\mathrm{A}$ and $\mathrm{C}$ ) control membrane, (B and D) 0.9$10 \mathrm{HNS}$, and (E) 0.9-20HNS.

It is well established that a successful antimicrobial surface could be identified by either direct kill of microbial cells, attachment inhibition of microbes or degradation of the extracellular polymeric substances (EPS) deposited during the biofilm formation (De Kwaadsteniet et al., 2011). Thus, the potential antibacterial activities of the nanocomposite membranes were measured and expressed by Log kill as shown in (Figure 6.9). Two microorganisms (E. coli and S. aureus), as a model bacterial species 
Chapter Six - 6 Fabrication of antibacterial nanocomposite membranes enabled by hybrid nanostructure modulated by microwave irradiation.

were used along with a standard testing protocol (ISO 20743) for membranous material. No apparent kill was anticipated with unmodified PES membrane against both species. As expected the viability of bacteria decreased with increasing HNS concentration in the nanocomposites. This biocidal property of the nanocomposites reached its maximum capability at $0.9 \% 10 \mathrm{HNS}$ concentration with 4.24 and 2.9 Log kill against E. coli and S. aureus, respectively. E. coli was more sensitive to Ag than S. aureus. This difference in susceptibility is argued to be due to the cell wall thickness, with the cell wall of Gram-positive bacteria being thicker than that of Gram-negative bacteria (Roy et al., 2015). The influence of nanobiocide size on the antibacterial activity of the nanocomposites cannot be disregarded. The smaller AgNPs found in nanocomposites prepared with $10 \mathrm{HNS}$ had higher activity than those prepared with $20 \mathrm{HNS}$ against EC, where Log kill values were 3.8 for $0.9-20 \mathrm{M}$ compared to 4.24 for $0.9-10 \mathrm{M}$ nanocomposites (Figure 6.10). This could be due to the higher surface to volume ratio of silver in the $10 \mathrm{HNS}$, which can display higher interactions with microorganisms and with silver supporting nanotubes.

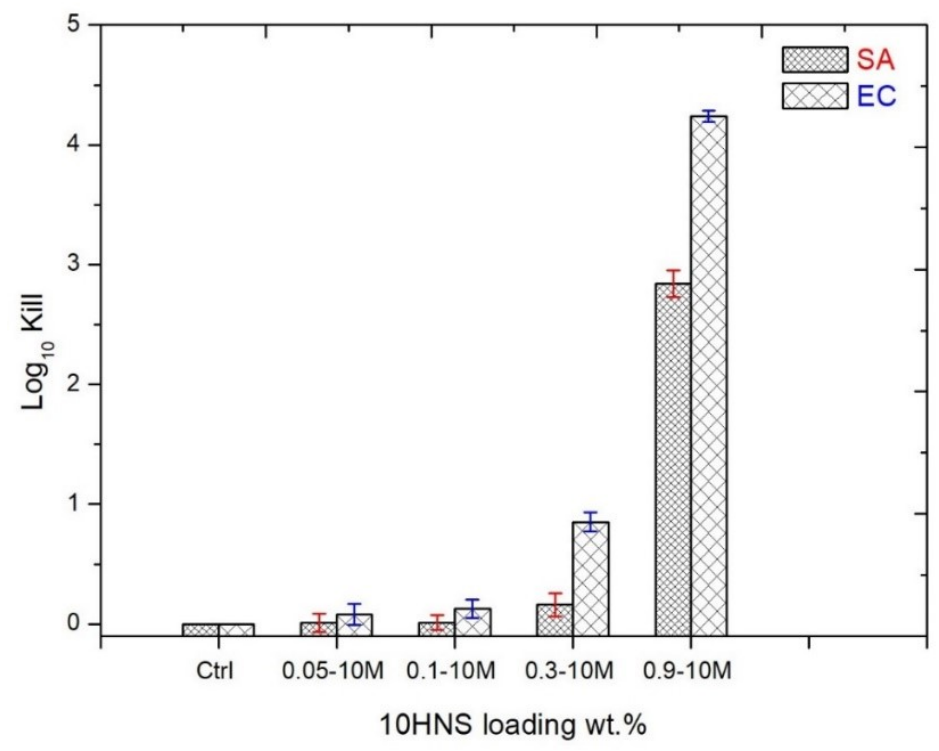

Figure 6.9: Bactericidal activities of nanocomposites against E. coli and S. aureus. 
Chapter Six - 6 Fabrication of antibacterial nanocomposite membranes enabled by hybrid nanostructure modulated by microwave irradiation.

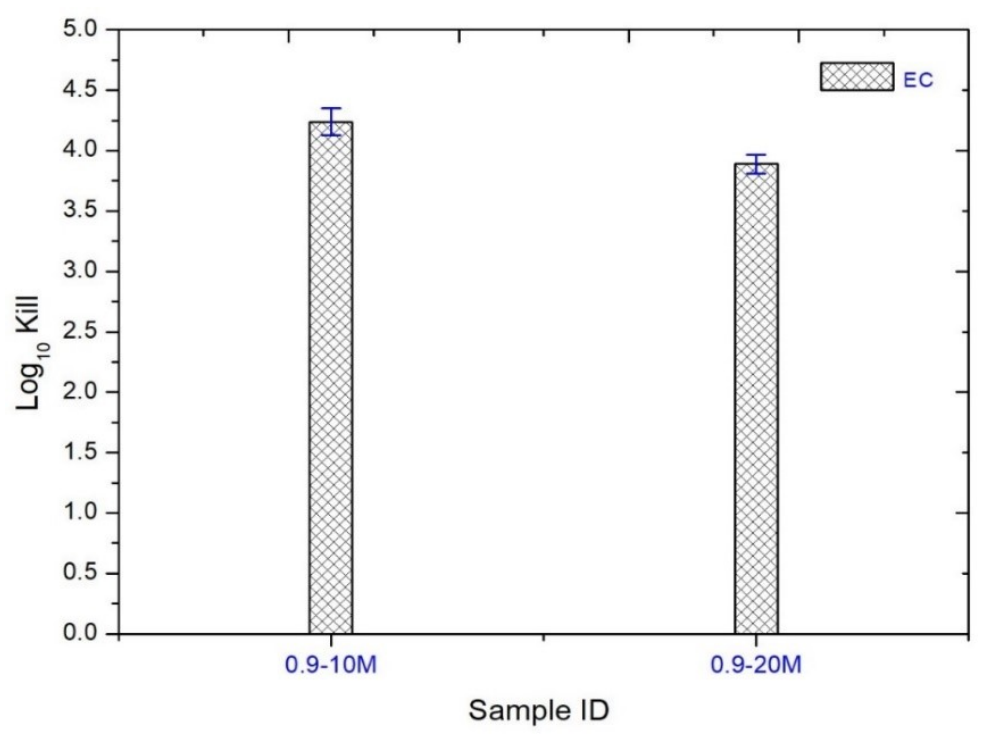

Figure 6.10: Variation in the antibacterial activity against E.coli for nanocomposites prepared using $10 \mathrm{HNS}$ and $20 \mathrm{HNS}$ at 0.9 loading $\mathrm{wt} \%$ to the polymer.

\subsection{Morphological characterisation of microorganisms}

The disruption of cell integrity of both microbial species induced by HNS was imaged by SEM. Membrane samples and inoculums $\left(1.0 \times 10^{6} \mathrm{CFU} / \mathrm{ml}\right)$ were prepared and cultured as reported in the ISO test. Briefly, membrane samples were sterilised with $70 \%$ ethanol-DI water solution for $10 \mathrm{~min}$ and left to dry under sterilised conditions using fume hood with UV lamp. Each sample was then placed in a sterilised falcon tube $(50 \mathrm{ml})$ and $200 \mu \mathrm{l}$ of inoculum was spread on the surface by disposable loops. Thereafter, samples were incubated for $24 \mathrm{hr}$ at $37{ }^{\circ} \mathrm{C}$. For fixation of the bacteria on membrane surfaces, all samples were immersed in $4 \mathrm{ml}$ glutaraldehyde ( $3 \% \mathrm{v} / \mathrm{v})$ and placed in a fridge overnight at $4{ }^{\circ} \mathrm{C}$. Later, samples were rinsed with PBS twice before the dehydration process. Dehydration was carried out using sequential concentrations $(25 \%, 50 \%, 75 \%$ and $100 \%)$ of ethanol (15 min each). Finally, before SEM imaging samples were coated with chromium by sputter coating (Quorum, Q150T ES, UK).

Surface morphology of both membranes and cells were examined using SEM, (Figure 6.11 and Figure 6.13). A smooth and flat surface can be observed for the control PES membrane, as can be seen in Figure 6.11A. However, impregnation of both HNS have manifested slight apparent roughness, as can be observed in (Figure 6.11B and Figure 6.11C). Furthermore, it should be noted that HNS was not observed on the surface of nanocomposites even though they moved up to the surface during the phase 
Chapter Six - 6 Fabrication of antibacterial nanocomposite membranes enabled by hybrid nanostructure modulated by microwave irradiation.

inversion, as depicted in Figure 6.1. This suggests that they have been entrapped within the polymeric matrix close to the top surface. Thus, there would have been no direct contact with the bacterial cells. However, a trial was also made to prepare nanocomposite membrane with only AgNPs impregnated in the polymeric matrix (Figure 6.12). As shown in the figure, AgNPs were apparent at the surface of the membrane, unlike other HNS. A closer look at the surface reveals uneven distribution with some microsize aggregation. These NPs were unable to withstand the cross-flow filtration conditions and the majority of NPs were leached out of the membranes after several runs.

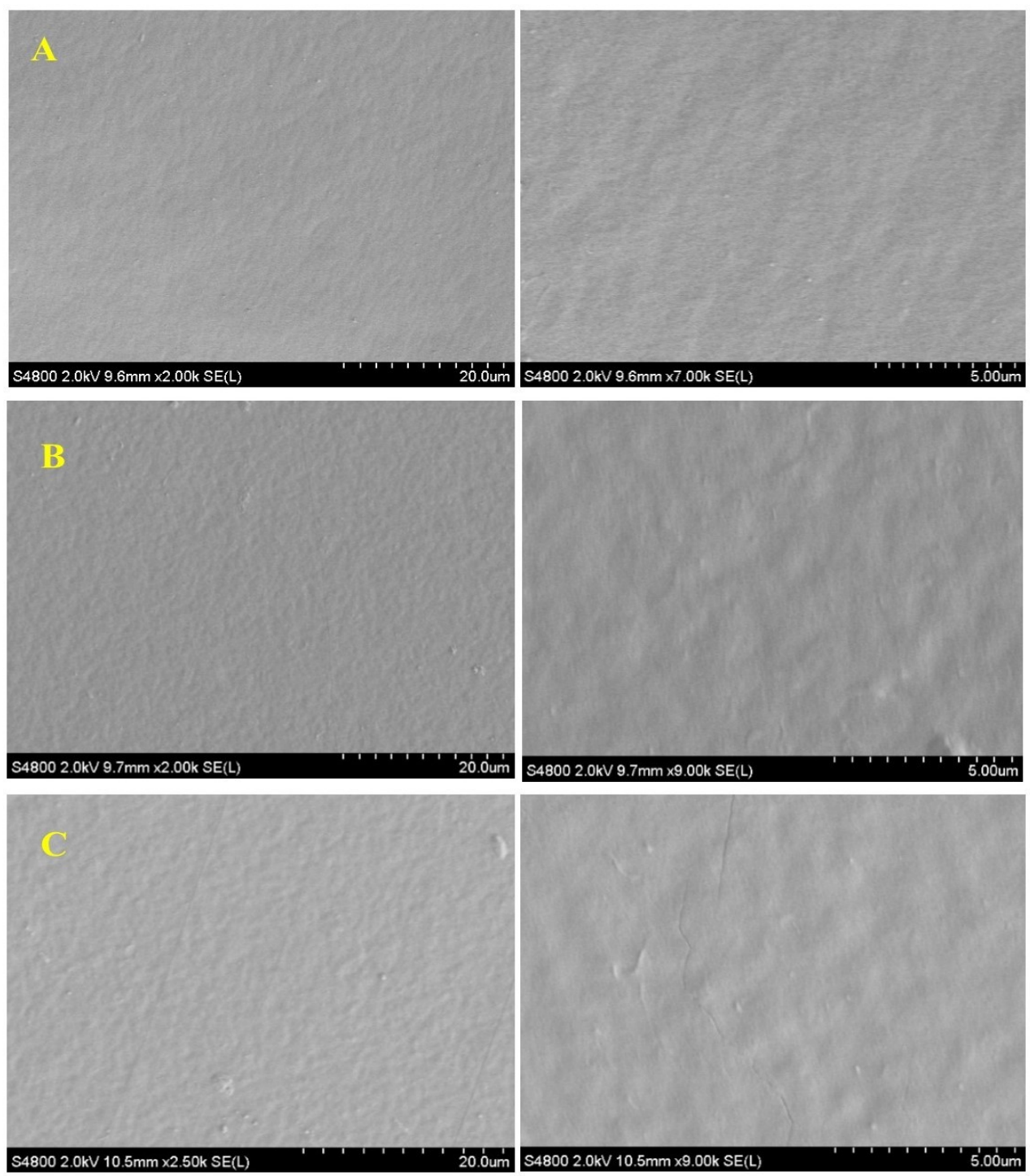

Figure 6.11: $5 \mu \mathrm{m}$ and $20 \mu \mathrm{m}$ SEM micrographs of (A) virgin control PES membrane, (B) virgin 0.9-10M nanocomposite, (C) Virgin 0.9-20M nanocomposite. 
Chapter Six - 6 Fabrication of antibacterial nanocomposite membranes enabled by hybrid nanostructure modulated by microwave irradiation.
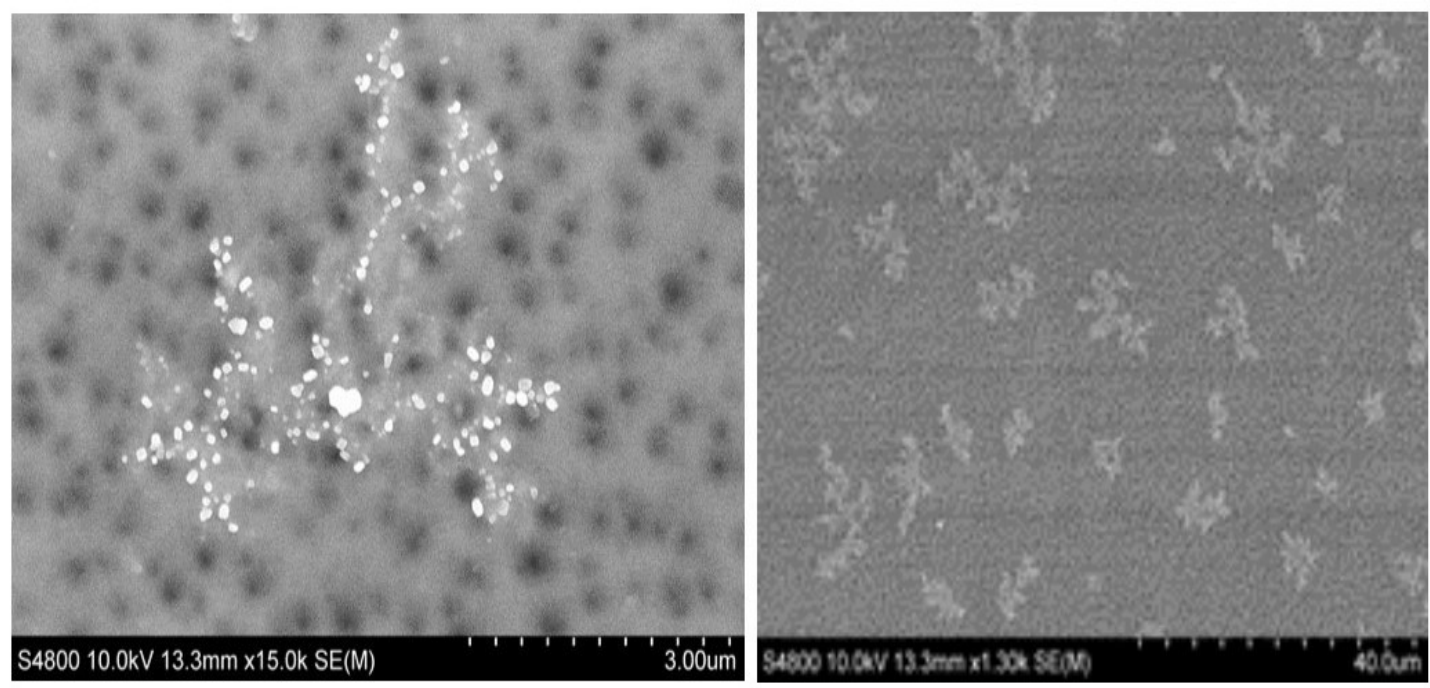

Figure 6.12: Surface SEM image for AgNPs/PES nanocomposite membrane. $3 \mu \mathrm{m}$ (Left) and $40 \mu \mathrm{m}$ (Right).

Both microorganisms were firmly colonised on the surface of unmodified PES membranes, and the surface topography revealed smooth, intact, rod and spherical shaped cells for E. coli and S. aureus, as shown in (Figure 6.13A and Figure 6.13C), respectively. However, the nanocomposites greatly influenced the cell wall morphology as confirmed by the markedly damaged and wrinkled surfaces of E. coli and S. aureus cells, (Figure 6.13B and Figure 6.13D), respectively. It is worth noting that following the treatment, the bacteria surface turned into rough cells while plentiful cytoplasmic substances were released due to the deterioration of the cell membrane. The deformation of the cell's membrane and loss of the original bacterium structure may be attributed to $\mathrm{Ag}^{+}$release. 
Chapter Six - 6 Fabrication of antibacterial nanocomposite membranes enabled by hybrid nanostructure modulated by microwave irradiation.
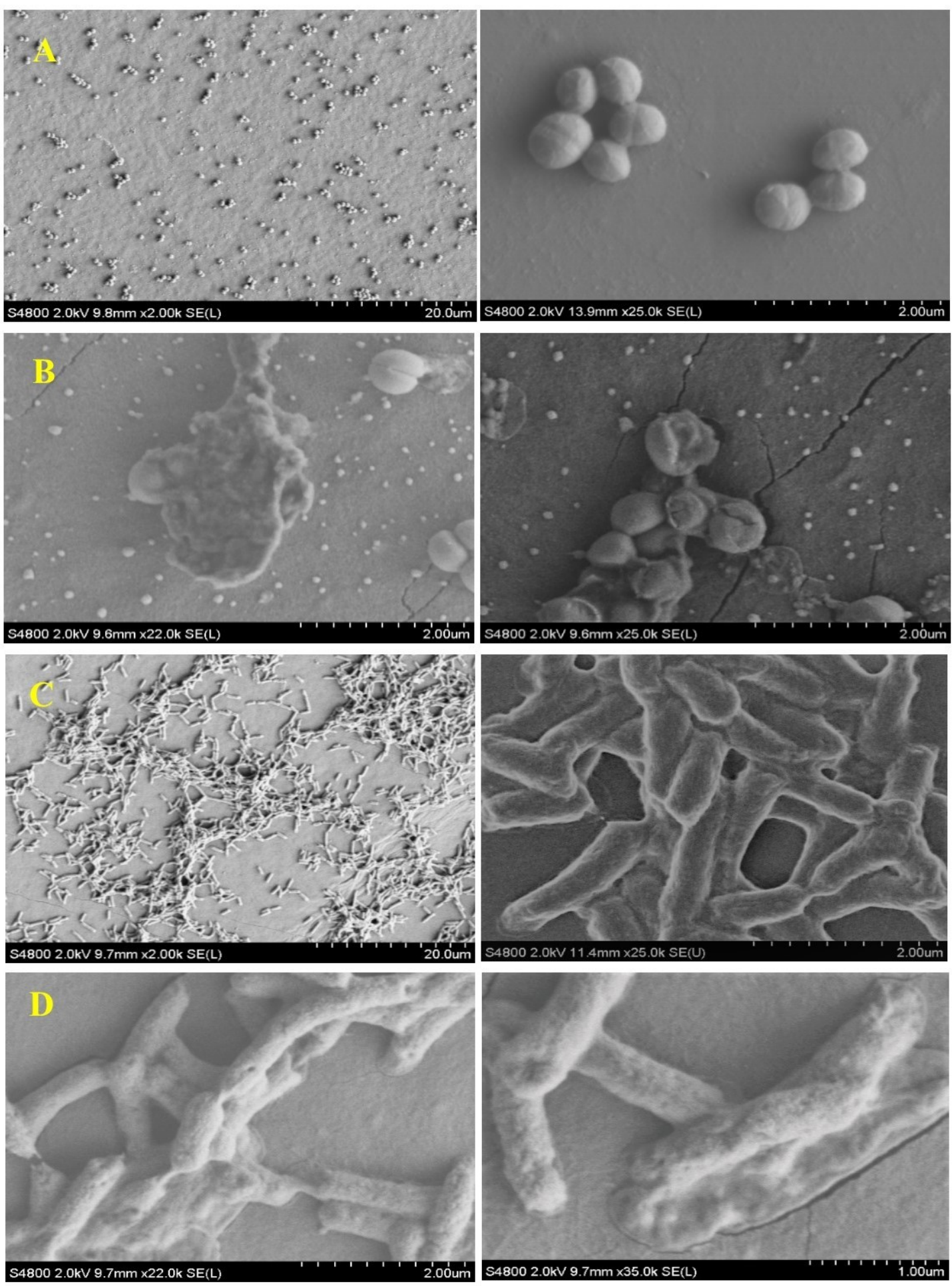

Figure 6.13: (A) control PES membrane fouled with S. aureus, (B) 0.9-10M nanocomposite fouled with $S$. aureus, (C) control PES membrane fouled with $E$. coli and (D) $0.9-10 \mathrm{M}$ nanocomposite fouled with $E$. coli. 
Chapter Six - 6 Fabrication of antibacterial nanocomposite membranes enabled by hybrid nanostructure modulated by microwave irradiation.

\subsection{Conclusions}

This work developed a novel membrane material for engineered nanocomposite membrane applications, based on design and fabrication of a facile, rapid and scalable biocidal HNS. 10 and 20 wt.\% of Ag/MWCNTs ratio were impregnated within a PES membrane matrix at different compositions to prepare two sets of nanocomposite membranes. Prior to fabrication, the anti-bacteriostatic of the 10HNS and 20HNS were confirmed by a zone of inhibition test using the representative species $E$. coli and $S$. aureus. Also, the impact of Ag/MWCNTs ratio and their content on the nanocomposites morphologies, surface characteristics, and antibacterial properties has been characterized. The content of HNS within a PES membrane matrix was found to have little influence on the surface pore size and pore size distribution if compared with the control membrane. However, the addition of the HNS to form a nanocomposite membrane had a significant impact on hydrophilicity measurement. Increasing the content of HNS has reduced the contact angle from $64^{\circ}$ to about $50^{\circ}$ for $0.9-10 \mathrm{M}$ and 0.9-20M nanocomposite membranes. All nanocomposite membranes manifested an enhancement in the pure water flux. Interestingly, even though the PWF returned to a lower value at the 0.9 wt.\%, it was still comparable to that of unmodified PES membrane. The surface zeta potential of all modified and unmodified membranes was found to be negatively charged for the entire $\mathrm{pH}$ range. Also, increasing the HNS loading to the PES has lowered the negative zeta potential value of nanocomposites. Interestingly, the presence of HNS was not visible even by high-resolution SEM images, which suggest that the HNS was immobilised within the polymeric chains close to the top surface of the nanocomposites. The potential antibacterial activities of the nanocomposite membranes were evaluated using ISO 20743 test and expressed by Log kill. This biocidal property of the nanocomposites reached its maximum capability at $0.9 \% 10 \mathrm{HNS}$ concentration with 4.24 and 2.9 Log kill against E. coli and S. aureus, respectively. The influence of nanobiocide size on the antibacterial activity of the nanocomposites cannot be disregarded. The smaller AgNPs found in nanocomposites prepared with $10 \mathrm{HNS}$ had higher activity than those prepared with $20 \mathrm{HNS}$, where Log kill values were 3.8 for $0.9-20 \mathrm{M}$ compared to 4.24 for $0.9-10 \mathrm{M}$ nanocomposites 


\section{Chapter Seven}

\section{$7 \quad$ Thin Film Nanocomposite (TFN) membranes modified with polydopamine coated metals/carbon-nanostructures for desalination applications}

\subsection{Introduction}

Since the first Thin Film Composite (TFC) membrane was made by (Cadotte et al., 1980), to the present day, a wealth of research has targeted improving TFCs for a breakthrough in industrial membrane expansion for desalination applications. TFC, which is viewed as the "golden standard" in the membrane industry, is constructed by an interfacial polymerisation of an organic phase-liquid phase system to form a thin $(0.1-1 \mu \mathrm{m})$ selective polyamide layer (PA) deposited on a microporous support membrane. This standard polymeric membrane has been the topic of intensive research in recent years in order to bring about distinctive features to the TFC surface. Novel materials and surface modification methods have been adopted for designing what is believed to be the next generation of TFC membrane. One of the recent pragmatic approached in this research has been achieved through revising the performance characteristics of TFC via imparting the specific characteristics of nanoscale materials (NMs) into the thin PA layer.

The recent concept "Thin Film Nanocomposite (TFN)", which was first reported in 2007 by (Jeong et al., 2007), refers to the application of nanoscale materials within or at the top surface of a PA layer. Since that time, a range of metal/metal oxides, zeolite, and carbon-based NMs have been reported in the literature, and reveal an 
Chapter Seven - Thin Film Nanocomposite membranes modified with polydopamine coated metals/carbon-nanostructures for desalination applications.

enhancement in TFN membranes functions. The enhancement was not only targeted at the antifouling properties of nanocomposites but also manifested outstanding capabilities to cope with the selectivity/permeability trade-off relationship (Lau et al., 2015). These nano-based structures could be either added to the aqueous phase or to the organic phase during the fabrication reaction of the PA layer or chemically bonded to the surface of TFC via bonding agents. However, gathering between a defect-free and stable PA layer is critical for long-term durability and stable selectivity of any TFN membrane. Regardless of the role of monomer's material, concentration and reaction time on the PA layer characteristics, other factors could considerably influence the performance of TFN, such as; type and size of fillers, the degree of dispersion/aggregation and quantity incorporated into the PA layer, and polymer-filler compatibility. Size and type of nanofiller can affect the permeability characteristics of TFN, for instance, big nanoparticle clusters or inappropriate alignment of nanotubes may act as defect sites in the TFN layer and give rise to a lower selectivity (Baroña et al., 2013; Lau et al., 2015). The relatively weak dispersion of hydrophilic NMs in nonpolar solvents is another key problem for the approach in TFN formation steps where most research focuses on their dispersion in the aqueous phase (Wu et al., 2010). Although considerable dispersion of surface modified NMs in the liquid phase can be achieved, agglomeration of NMs is still unavoidable, especially at high loading weight. Nevertheless, lack of surface interactions between the PA matrix and NMs are more likely to result in leaching of NMs out of the PA thin film during the formation and/or filtration, affecting the selectivity and stability of the TFN membranes (Lau et al., 2015).

Currently, attempts are directed to modify the conventional IP process and/or to link diverse organic derivatives on NMs surface, aiming to revise their surface functionalization and promote their colloidal stability in various media. A new technique was introduced by Baroña and co-worker to develop TFN membrane for lowpressure RO membrane, where a single pass flow was used to incorporate aluminosilicate- single-walled carbon nanotubes (SWCNTs) in a PA layer (Baroña et al., 2013). Yin J. et al., have employed ethanol to facilitate the dispersion of graphene oxide (GO) in the TMC-hexane solution for PA thin-film layer formation (Yin et al., 2016). While, Rajaeian et al., has utilised N-[3-(trimethoxysilyl) propyl] ethylenediamine (AAPTS) as a silane coupling agent to revise the surface 
Chapter Seven - Thin Film Nanocomposite membranes modified with polydopamine coated metals/carbon-nanostructures for desalination applications.

characteristics of $\mathrm{TiO} 2$ before addition to an amine-aqueous solution for TFN/NF membrane fabrication (Rajaeian et al., 2013). Rajaeian et al., assumed that silane functional groups could minimise agglomeration of NPs throughout minimising the probability of oxygen bridge bonds formation between $\mathrm{TiO}_{2} \mathrm{NPs}$.

One of the solutions for this is the use of dopamine (DA). DA as a mussel-inspired "bio-glue" has been widely used for surface modification in a wide range of applications includes surface coating, filtration, chemical sensing, biomedicine, and energy storage. The Structure of PDA is still under discussion but mainly composes dihydroxyindoline, indolinedione, and eventually dopamine units are held together by hydrogen bonding between oxygen atoms or $\pi$ stacking (Liebscher et al., 2013). Under an alkaline environment, DA can be self-polymerised to form a polymerlike coating layer of polydopamine (PDA) with extraordinary potential adhesive strength for a variety of organic and inorganic materials, e.g. polymers, noble metals, oxides, semiconductors and ceramics (Zhang et al., 2015). PDA-coated surfaces can be further functionalized with different molecules through the catechol and quinoid units to create desired functionality (Orishchin et al., 2017). PDA cannot only enhance the dispersion of nanofillers in aqueous solutions but also consolidate the surface interactions between PA matrix and NMs (Lv et al., 2017). Lv Y. et al., identified that PDA can promote the interfacial stability between polymers and inorganic materials through acting as a bridge to chelate metal oxides for constructing inorganic films on various substrates (Lv et al., 2016).

In this research, a very novel approach has been introduced for TFN membrane fabrication. Instead of dispersing a nanofiller in the aqueous or organic phase aiming to embed them in the constructed PA layer, this introduces a thin hydrophilic intermediate layer of HNS between the substrate membrane and PA skin layer. This HNS comprised of metal/metal oxide NPs coated multiwalled carbon nanotubes (MWCNTs). Four different metal/metal oxide $\left(\mathrm{Ag}, \mathrm{Al}_{2} \mathrm{O}_{3}, \mathrm{Fe}_{2} \mathrm{O}_{3}\right.$, and $\left.\mathrm{TiO}_{2}\right)$ were first deposited on the nanotube surfaces then coated with a PDA layer to get the final product. Next, a thin layer of HNS was deposited on a porous substrate membrane by vacuum filtration and the PA layer was adjusted to entirely cover and robustly fix the HNS interlayer in order to produce a defect-free TFN membrane. This method resulted in a low incorporation of NMs and avoided their wastage during the TFN preparation. Vacuum pressure was applied instead of a rubber roller to remove the excessive aqueous solution and to avoid 
Chapter Seven - Thin Film Nanocomposite membranes modified with polydopamine coated metals/carbon-nanostructures for desalination applications.

any possible disruption or detachment of NMs layer that the rubber roller might cause during the preparation. This would also avoid any internal blockage of pores that could result during the PA formation, and lead to lower permeation characteristics. No appreciable amount of NMs would be wasted in order to incorporate a certain wt $\%$ of NMs, as has been detected in the conventional TFN fabrication techniques. To the best of authors knowledge, no attempt has been made to use such novel hybrid nanostructure (HNS), comprising of CNTs-M/MO within the thin PA skin layer, and/or compared to the function of different types of HNS on the TFN characteristics.

\subsection{Synthesis of hybrid nanostructured materials}

The four-different metal/metal oxide $\left(\mathrm{Ag}, \mathrm{Al}_{2} \mathrm{O}_{3}, \mathrm{Fe}_{2} \mathrm{O}_{3}\right.$ and $\left.\mathrm{TiO}_{2}\right)-$ MWCNTs based nanocomposites, employed for this part of the thesis, have been prepared according to the detailed procedure reported in section 5.2, except coating the nanostructure with the thin layer of PDA which can be described as follows; a bath sonicator was used to suspend $50 \mathrm{mg}$ of each HNS sample in $100 \mathrm{ml}$ Tris-HCl buffer $(10 \mathrm{mM}$ and $\mathrm{pH} 8.5)$. Later, about $75 \mathrm{mg}$ dopamine was added and stirred at $25{ }^{\circ} \mathrm{C}$ for $24 \mathrm{~h}$. After polymerisation, the PDA coated nanotubes were washed several times with DI water to neutralise the $\mathrm{pH}$. Finally, the product was dried under vacuum for overnight. An example of the prepared HNS is presented in Figure 7.1 below.
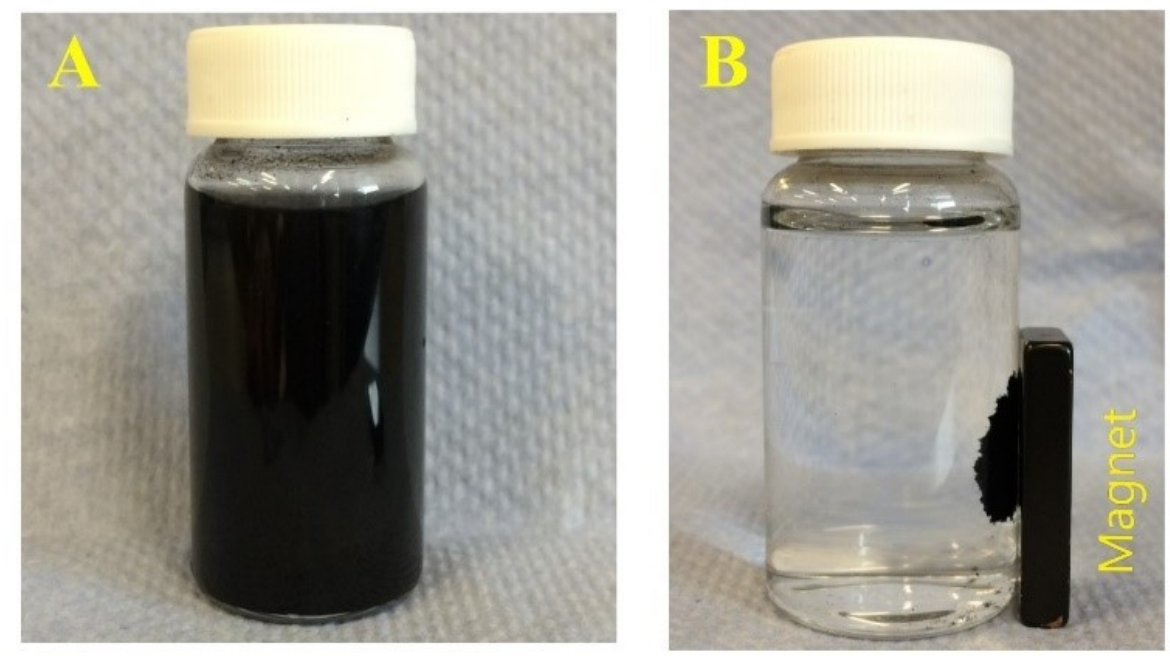

Figure 7.1: Photographic view of $\mathrm{Fe}_{2} \mathrm{O}_{3}-\mathrm{CNT}$ s nanocomposites: (A) dispersed in water, (B) their response to a magnetic field. 
Chapter Seven - Thin Film Nanocomposite membranes modified with polydopamine coated metals/carbon-nanostructures for desalination applications.

\subsection{Fabrication of thin film nanocomposite (TFN)}

Laboratory-made PES/UF membranes were prepared by the classical wet phase inversion and used as a substrate for the polyamide layer polymerisation. A more detailed fabrication description of the procedure is presented in section 3.3.1. Briefly, $14 \mathrm{wt} \%$ PES and 2 wt \% PVP were dissolved in NMP at $50{ }^{\circ} \mathrm{C}$ overnight, until a clear yellowish solution was achieved. The casting solution was then degassed for $1 \mathrm{~h}$ under vacuum prior to casting. Next, membranes were cast on a nonwoven support (AhlstromHollytex ${ }^{\circledR}$ - grade:3329) using a thin film applicator with a clearance gap of $200 \mu \mathrm{m}$. Thereafter, the substrate was directly placed in a DI water coagulation bath at $20^{\circ} \mathrm{C}$ for $30 \mathrm{~min}$, washed several times, and stored in an airtight DI water container at $4{ }^{\circ} \mathrm{C}$ ready for further use.

TFC and TFN membranes were fabricated via interfacial polymerisation on the membranes prepared earlier. For TFC membrane, a rectangular cut membrane was first clamped between a rectangle frame $(20 \mathrm{~cm} \times 5 \mathrm{~cm})$ and a substrate made from acrylic (Figure 7.2) and connected to a vacuum unit. About $50 \mathrm{ml}$ of $2 \% \mathrm{MPD}(\mathrm{w} / \mathrm{v})$ in water solution was left in contact with the active membrane's surface for $2 \mathrm{~min}$, followed by applying a vacuum pressure to remove the excess solution. Then, $50 \mathrm{ml}$ of $0.1 \% \mathrm{TMC}$ in $n$-hexane $(\mathrm{w} / \mathrm{v})$ was reacted with the top surface of the MPD wetted substrate for 45 s. After the IP process, the TFC was rinsed with about $50 \mathrm{ml}$-hexane to remove unreacted monomers, crosslinked in an oven at $70{ }^{\circ} \mathrm{C}$ for $15 \mathrm{~min}$, and finally stored in DI water prior to testing.

Except for one additional step, the same procedure was performed for the TFN fabrication. Following the clamping of the support membrane, a suspension of each PDA coated HNS was individually prepared and used to deposit $0.0025 \mathrm{mg} / \mathrm{cm}^{2}$ on the support membrane by vacuum filtration, forming a very thin layer of HNS on the top surface. The MPD was then poured carefully to wet the deposited thin layer for $2 \mathrm{~min}$. Then, vacuum pressure was applied again to remove excess solution from the surface and to wet the internal wall of pores. As mentioned in TFC procedure, TMC was then reacted with the amine solution for $45 \mathrm{~s}$, rinsed with n-hexane, crosslinked at $70{ }^{\circ} \mathrm{C}$, and stored in DI water ready for characterisation. 
Chapter Seven - Thin Film Nanocomposite membranes modified with polydopamine coated metals/carbon-nanostructures for desalination applications.
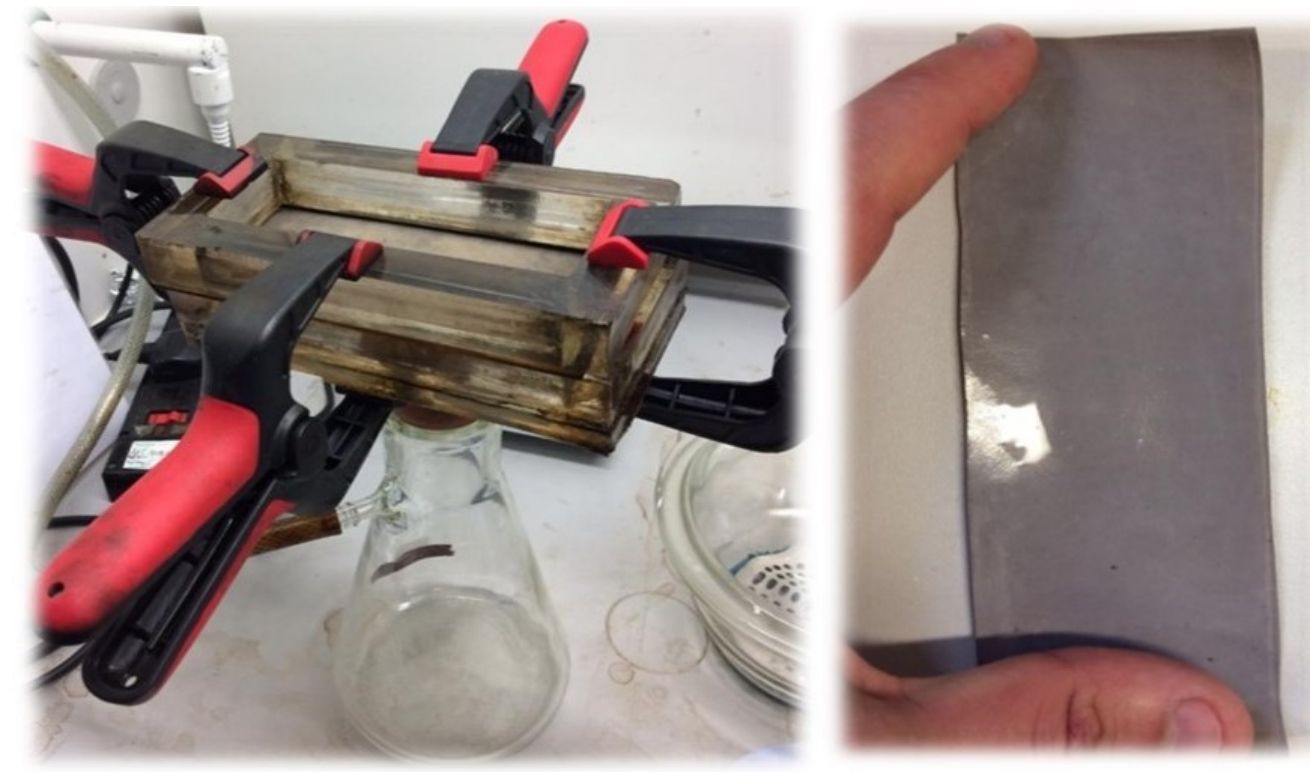

Figure 7.2: TFN membrane fabrication, acrylic frame and vacuum unit (left), and TFN membrane sheet (right).

\subsection{Characterization of PDA coated nanocomposite materials}

\subsubsection{FTIR spectra evaluation of MWCNTs}

FTIR has been employed to demonstrate the successful carboxylation of nanotubes and coating of HNS with the bioinspired PDA layer. The total reflection infrared spectra from 500 to $4000 \mathrm{~cm}^{-1}$ are shown in Figure 7.3 for raw, functionalized and PDA coated nanotubes. A clear enhancement in the partial oxidation of nanotubes was observed after treatment with concentrated $\mathrm{HNO}_{3}$ where a number of functional groups, mainly hydroxyl $(-\mathrm{OH})$ and carboxyl $(-\mathrm{COOH})$, are generated after the acid treatment of $\mathrm{r}$ CNTs. The FTIR spectra of r-CNTs did not reveal clear enough bands to be recognised. However, the broad well-defined peak around 2994-3691 cm $\mathrm{cm}^{-1}$, which is always presented for the nanotubes, is mainly attributed to the stretching mode of the $(-\mathrm{OH})$ bond. The IR regions around $1665 \mathrm{~cm}^{-1}$ are ascribed to oscillation of $-\mathrm{COOH}$ groups, while the small peak at $1362 \mathrm{~cm}^{-1}$ is the $\mathrm{O}-\mathrm{H}$ vibration bending of carboxyl acidic group (Moraes et al., 2011). The well-defined peak at $1060 \mathrm{~cm}^{-1}$ was correlated to the $\mathrm{C}-\mathrm{O}$ stretching vibration. All these identified peaks confirmed the successful modification of r-CNTs with acidic functional groups. Comparing the IR spectra of CNTs and other HNS, a new peak ascribed to pure metal oxide NPs was observed between $\sim 450$ and $700 \mathrm{~cm}^{-1}$, depending on the M/MO type. Following the PDA coating, 
Chapter Seven - Thin Film Nanocomposite membranes modified with polydopamine coated metals/carbon-nanostructures for desalination applications.

peaks at $1060 \mathrm{~cm}^{-1}, 1362 \mathrm{~cm}^{-1}$, and $1665 \mathrm{~cm}^{-1}$ have disappeared, and new peaks have emerged around $1272 \mathrm{~cm}^{-1}$ and $1572 \mathrm{~cm}^{-1}$ instead. These peaks are ascribed to $\mathrm{C}-\mathrm{O}$ stretching vibration of Phenol and $\mathrm{N}-\mathrm{H}$ vibration response of the amide II bands, respectively (Yang et al., 2015), which is considered as a confirmation of felicitous muscle inspired chemical packaging on the surface of HNS.

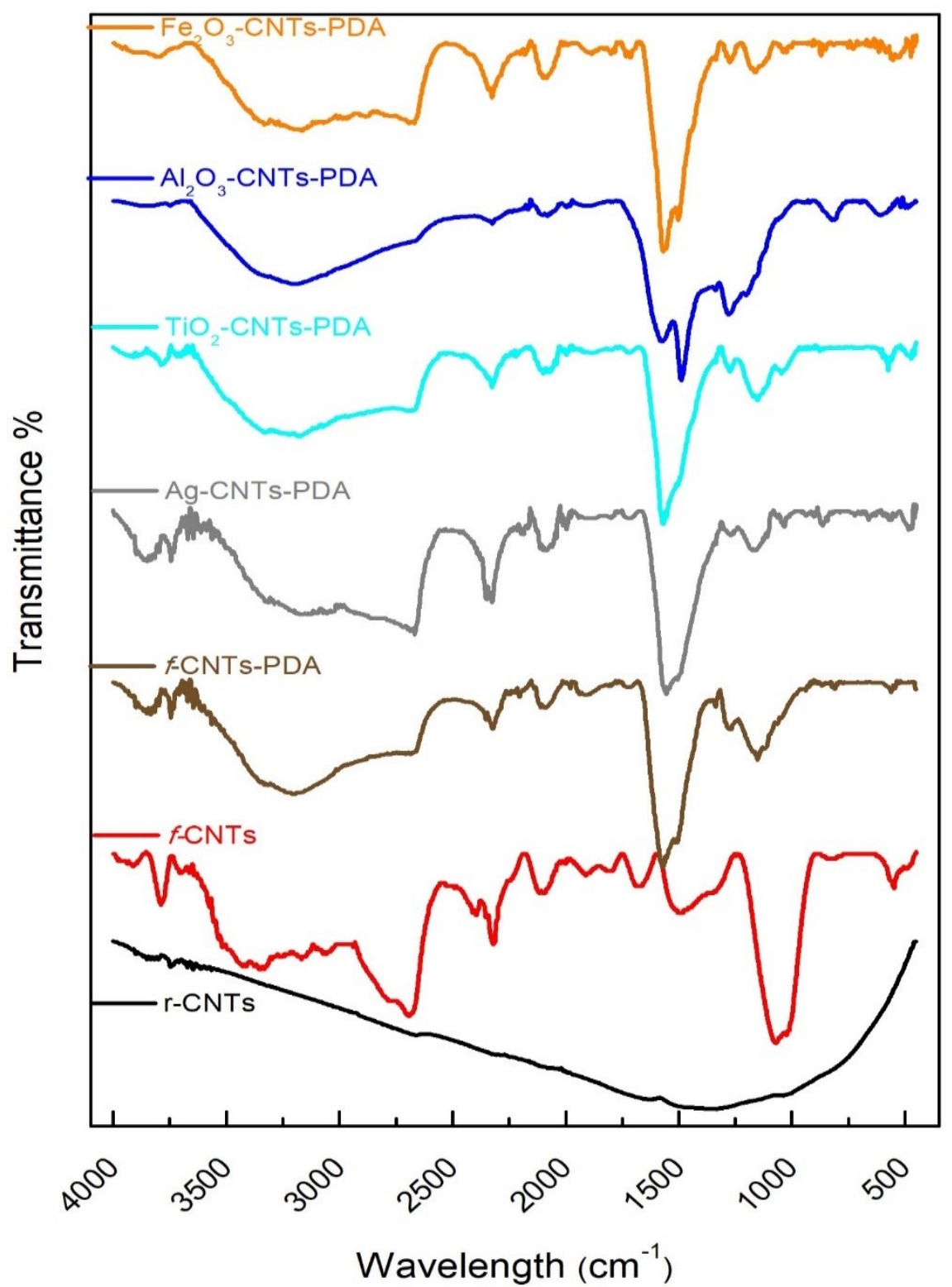

Figure 7.3: Fourier transform infrared spectroscopy (FT-IR) spectrum of r-CNTs, fCNTs, f-CNTs-PDA and PDA coated nanocomposites.

\subsubsection{Thermal behaviour analysis (TGA)}

TGA analysis was conducted to illustrate the thermal decomposition behaviour of various samples after coating with PDA. In addition, the extent of PDA grafting on $\mathrm{f}$ - 
Chapter Seven - Thin Film Nanocomposite membranes modified with polydopamine coated metals/carbon-nanostructures for desalination applications.

MWCNTs and other HNS was evaluated. The significant weight loss in the f-MWCNTs was between 547 and $673{ }^{\circ} \mathrm{C}$, as illustrated in Chapter 5 (Figure 5.12). At $547^{\circ} \mathrm{C}$, the mass loss of f-MWCNTs was only $12 \%$. While after coating with PDA, this weight loss reached $55 \%$, suggesting that $43 \%$ of PDA was successfully loaded on the nanotubes via mussel inspired chemistry, Figure 7.4. Similarly, the amount of grafted PDA on the surface of $\mathrm{Fe}_{2} \mathrm{O}_{3}-\mathrm{MWCNTs}$, $\mathrm{TiO}_{2}$-MWCNTs and $\mathrm{Al}_{2} \mathrm{O}_{3}$-MWCNTs was $34 \%$, 40\%, and $45 \%$, respectively. It should be noted that PDA slightly reduced the thermal stability of nanocomposites except that of Ag-MWCNTs. This was probably due to the decomposition of PDA that lies within (200-500) ${ }^{\circ} \mathrm{C}$ (Subramanian et al., 2016), the final decomposition temperature of the nanocomposite has shifted from $400{ }^{\circ} \mathrm{C}$ to 545 ${ }^{\circ} \mathrm{C}$, causing an improvement in thermal properties after PDA coating for Ag-MWCNTs samples only.

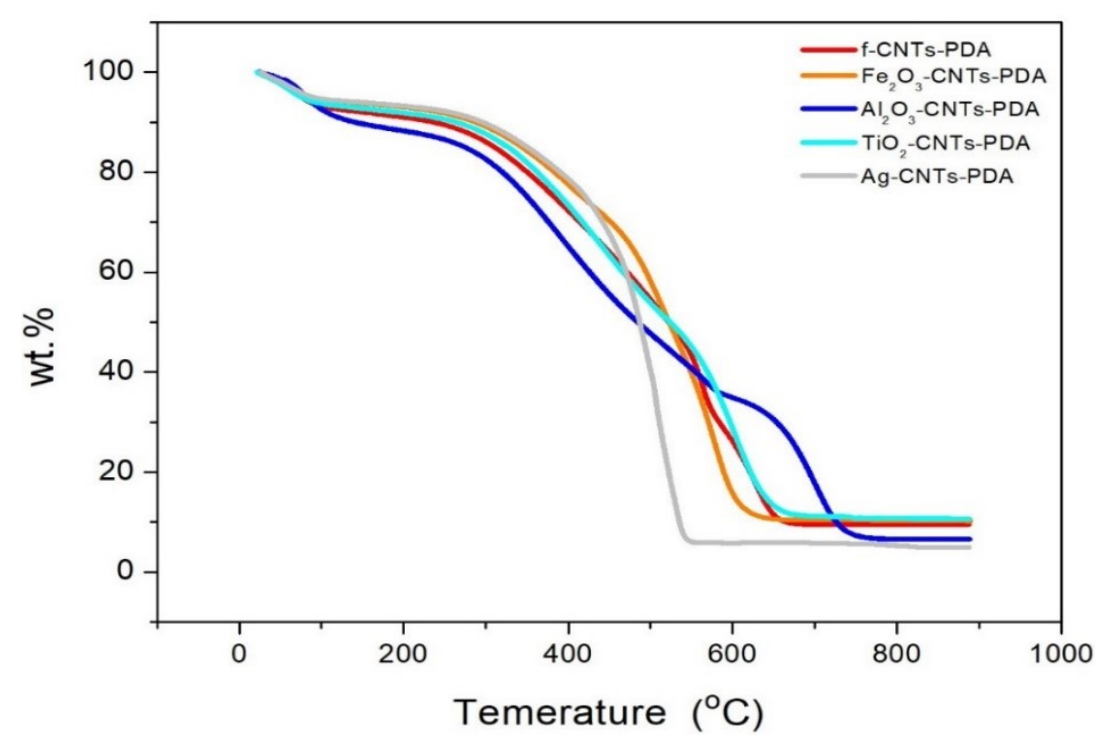

Figure 7.4: TGA curves in the air for PDA coated nanocomposites.

\subsubsection{Zeta potential measurements}

Zeta potential measurements were carried to assess the colloidal stability of the HNS after wrapping up with PDA layer. As can be seen, coating the nanocomposites with PDA caused supplemental charge reversal especially in an acidic environment (below $\mathrm{pH}$ 5.5), and a clear shift in the IEP was observed toward a higher $\mathrm{pH}$ value for all PDA coated HNS (Figure 7.5). This observation was due to the protonation of indole or indoline groups of PDA, which tends to neutralise the negative charge of HNS ( $\mathrm{Yu}$ et 
Chapter Seven - Thin Film Nanocomposite membranes modified with polydopamine coated metals/carbon-nanostructures for desalination applications.

al., 2014). At higher $\mathrm{pH}(\mathrm{pH}>6)$, all HNS commence featuring negatively charged surfaces.

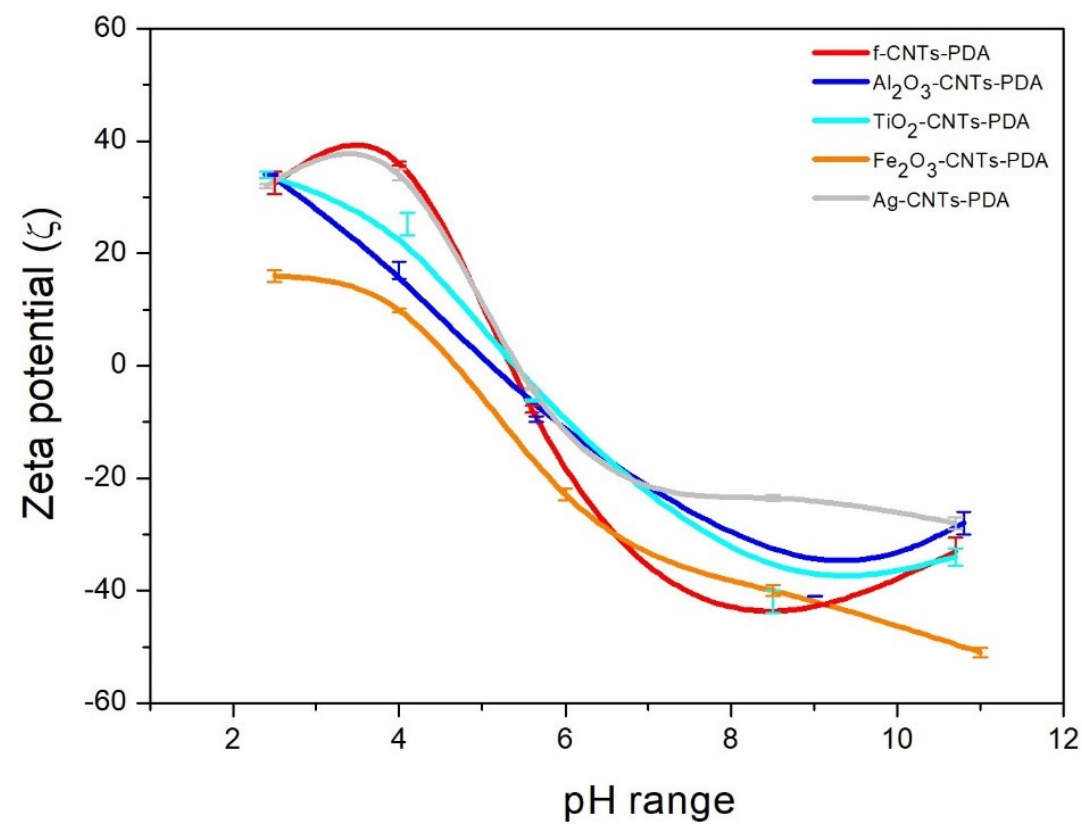

Figure 7.5: Zeta potential measurements as a function of $\mathrm{pH}$, for PDA coated nanocomposites.

\subsection{Characterisation of thin film nanocomposite membranes.}

\subsubsection{Infrared spectra (FT-IR) of membranes}

Infrared spectroscopy is one of the easy and direct potent tools for the characterisation of molecular bonds between chemical compounds of a membrane together with their possible functional groups, which might not be detectable by XPS (Hilal et al., 2017). In this section, the chemical structure of the PES substrate, TFC and TFN membranes skin layer was verified and compared by FTIR, as shown in Figure 7.6. Several peaks, associated with PES membrane, were observed around $1099 \mathrm{~cm}^{-1}, 1148 \mathrm{~cm}^{-1}, 1240$ $\mathrm{cm}^{-1}, 1337 \mathrm{~cm}^{-1}, 1485 \mathrm{~cm}^{-1}$ and $1578 \mathrm{~cm}^{-1}$, and correspond to $\mathrm{C}-\mathrm{O}$ stretching, $\mathrm{O}=\mathrm{S}=\mathrm{O}$ symmetric stretching, aromatic ether, $\mathrm{O}=\mathrm{S}=\mathrm{O}$ asymmetric stretching, $\mathrm{C}=\mathrm{C}$ stretching and benzene ring, respectively. More precisely, peaks found at $1485 \mathrm{~cm}^{-1}$ and 1578 $\mathrm{cm}^{-1}$ are the main characteristics of PES membrane material (Ingole et al., 2014). Following the interfacial polymerisation of the PA layer, the IR spectra of TFC contained a novel peak around $1666 \mathrm{~cm}^{-1}$. This peak is assigned to amide I band, a characteristic of $\mathrm{C}=\mathrm{O}$ bonds of an amide group. In addition, there was also a more intense peak at $1578 \mathrm{~cm}^{-1}$ assigned to amide II band for N-H (Baroña et al., 2013). 
Chapter Seven - Thin Film Nanocomposite membranes modified with polydopamine coated metals/carbon-nanostructures for desalination applications.

However, introducing the HNS as interlayers between the PES substrate and thin PA layer has influenced the intensity of some peaks (e.g. $557 \mathrm{~cm}^{-1} .1485 \mathrm{~cm}^{-1}, 1578 \mathrm{~cm}^{-1}$ and $1710 \mathrm{~cm}^{-1}$ ). The boosted intensity of the peak situated at $1710 \mathrm{~cm}^{-1}$, was mainly due to $\mathrm{C}=\mathrm{O}$ stretching vibration from $-\mathrm{COOH}$ groups of $\mathrm{HNS}$ (Wu et al., 2016). Moreover, the formation of amide bands was also influenced in all TFN types.

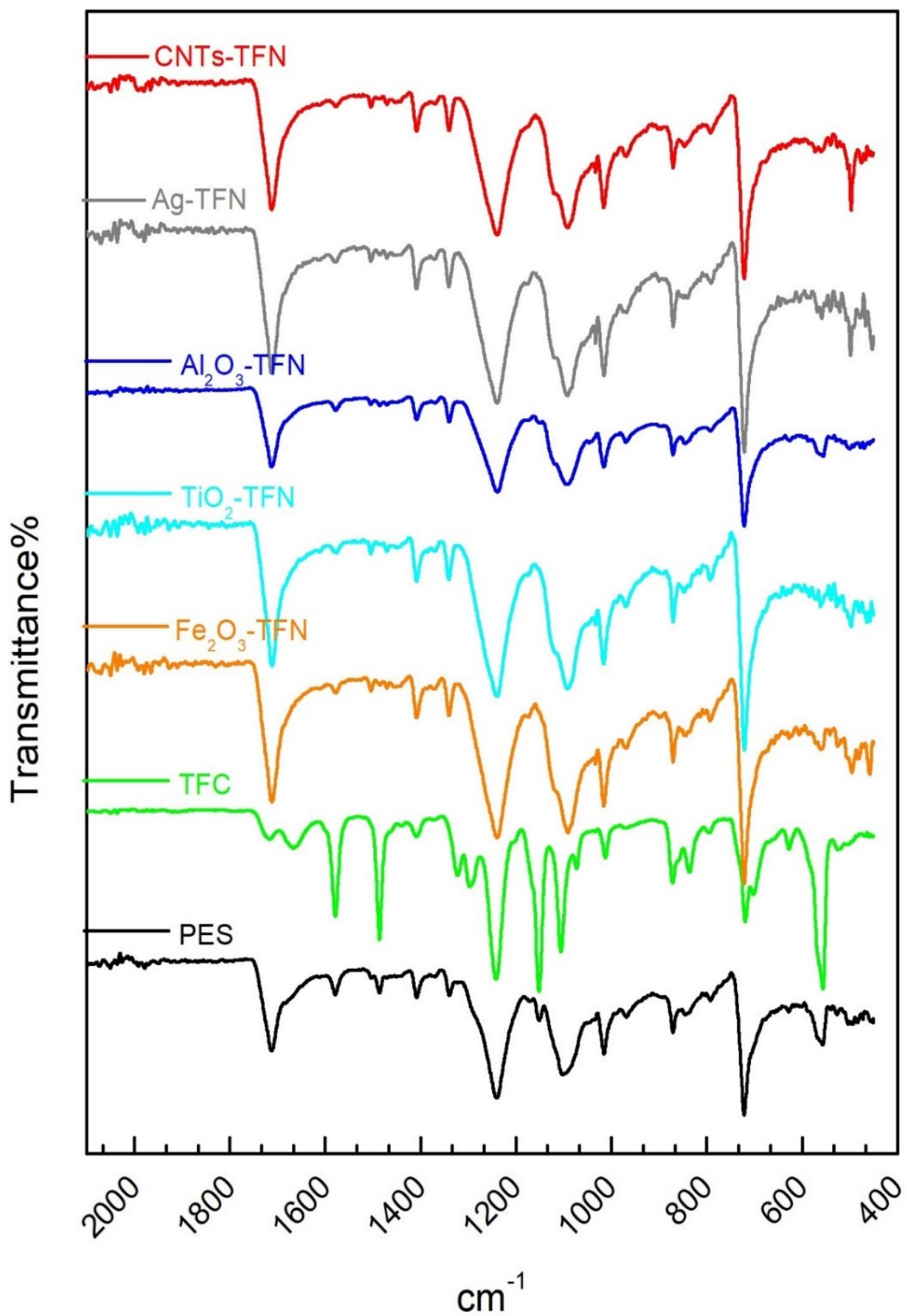

Figure 7.6: FT-IR spectra of the synthesised PES, TFC and TFN membranes. 
Chapter Seven - Thin Film Nanocomposite membranes modified with polydopamine coated metals/carbon-nanostructures for desalination applications.

\subsubsection{Morphology of TFN membranes}

The surface and cross-sectional morphology of TFC and TFN membranes were observed by FESEM, as presented in Figure 7.7 and Figure 7.8, respectively. The SEM surface images had the typical leaf-like structure of the PA layer. No explicit variation could be seen between TFC and TFN, which indicates that the PA layer has entirely covered the HNS interlayer, Figure 7.7A-F. While the cross-section images of all NF membranes have two distinct morphologies; the PA skin layer and the supporting PES substrate.
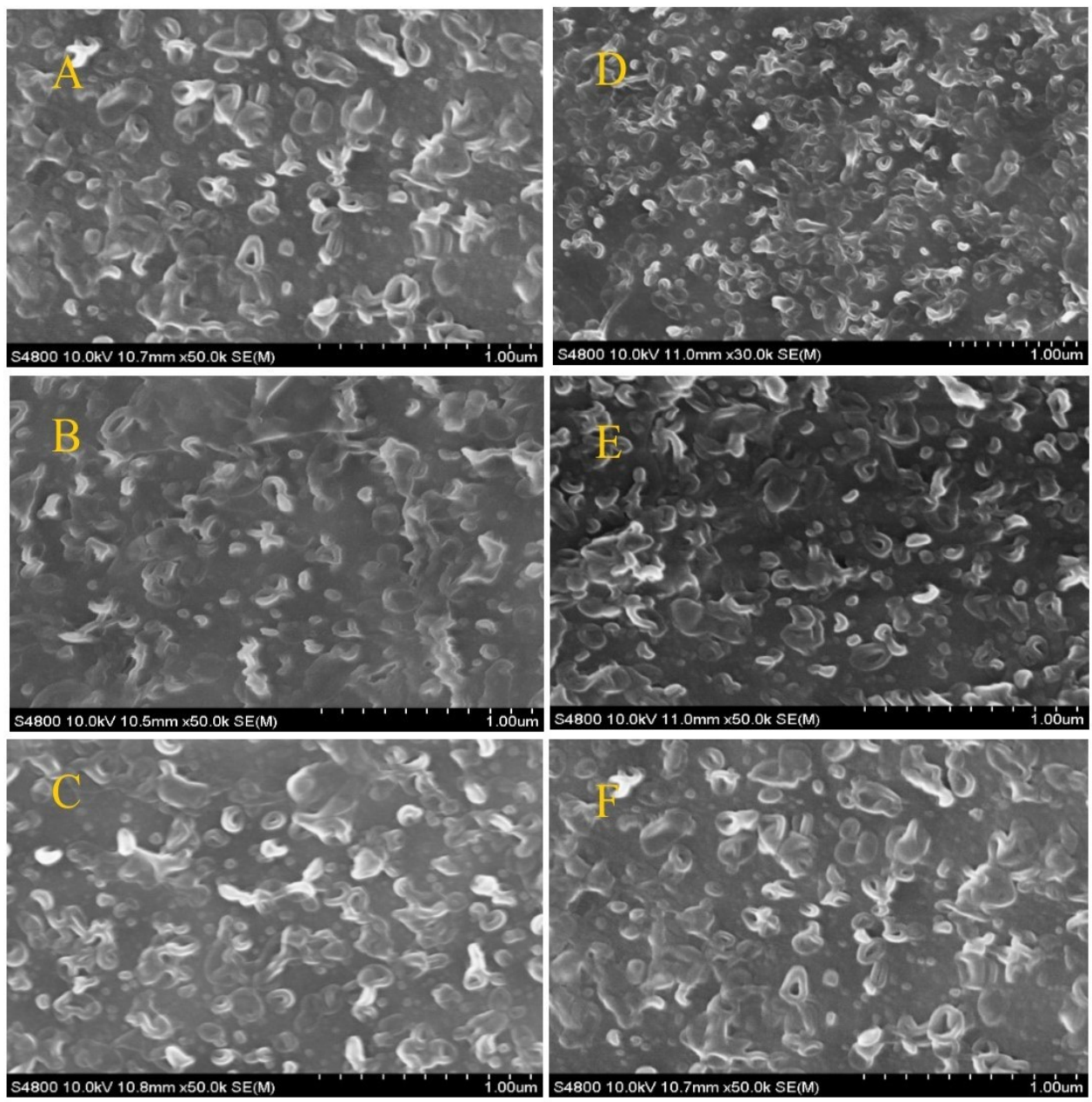

Figure 7.7: SEM surface images for; (A) TFC, (B) f-CNTs-TFN, (C) Ag-TFN, (D) $\mathrm{Al}_{2} \mathrm{O}_{3}$-TFN, (E) $\mathrm{Fe}_{2} \mathrm{O}_{3}-\mathrm{TFN}$, (F) $\mathrm{TiO}_{2}$-TFN.

The observed thickness of the PA thin-film layer in TFC membrane was ranging from $225 \mathrm{~nm}$ to $245 \mathrm{~nm}$, while the active layer of all other TFN had a higher thickness $(340 \pm 50 \mathrm{~nm})$ compared to that of TFC membrane. This augmentation in the thickness has been correlated to the existence of an HNS intermediate layer, which can absorb 
Chapter Seven - Thin Film Nanocomposite membranes modified with polydopamine coated metals/carbon-nanostructures for desalination applications.

and store the aqueous MPD solution to facilitate the subsequent interfacial polymerisation process ( $\mathrm{Wu}$ et al., 2016). As depicted in Figure 7.8A-F, the presence of HNS was not conspicuous in the SEM cross-section images. This could be ascribed to the low contrast between HNS and the PA thin film, especially if they were wrapped within the PA polymeric matrix. It is believed herein that the interlayer was built up inside the PA matrix, and the PA forming monomers were penetrated, via the applied vacuum, through the HNS interlayer to attach the PES membrane surface and internal pores walls in order to anchor the thin film on the PES substrate.
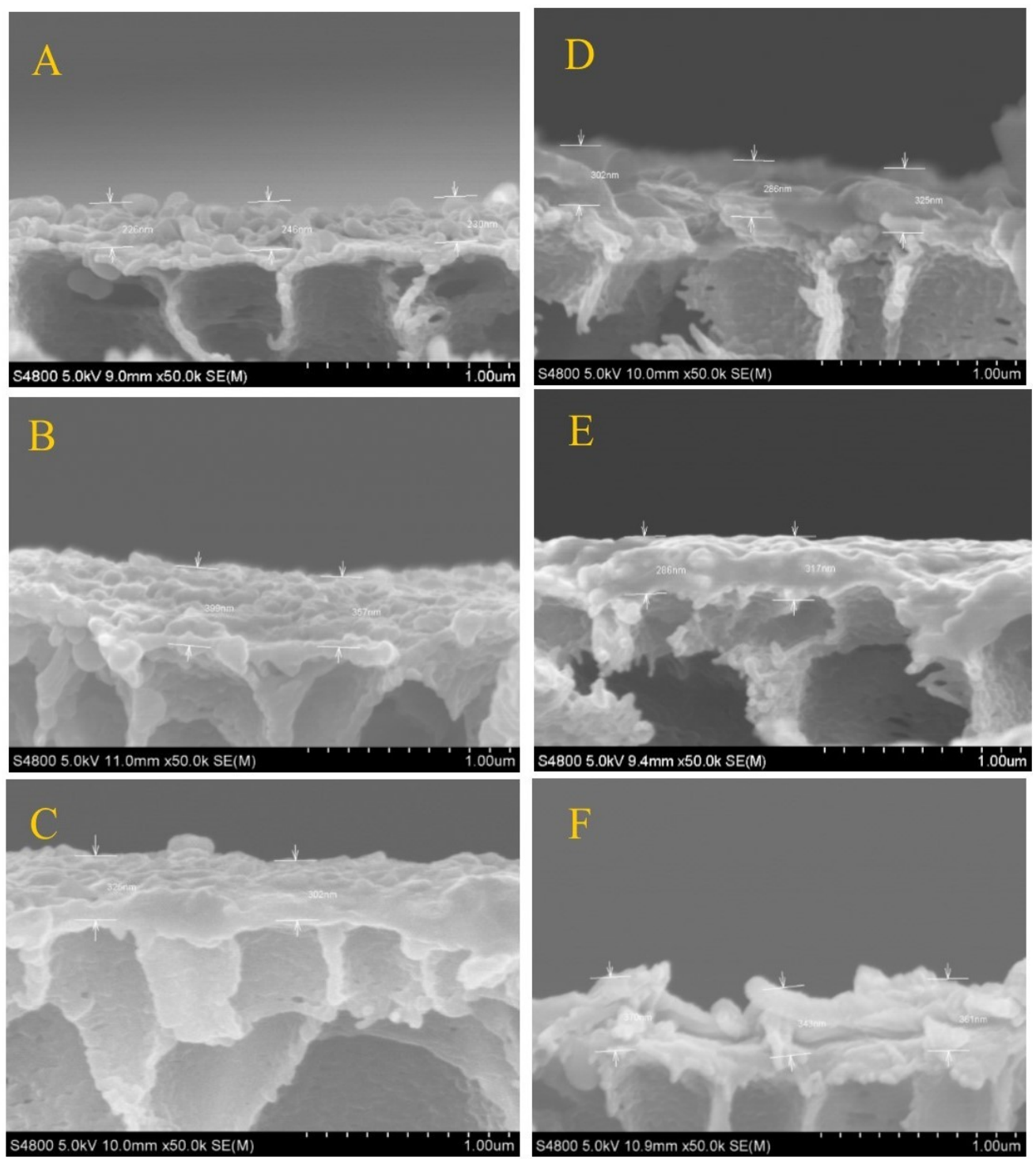

Figure 7.8: SEM cross-section images for; (A) TFC, (B) f-CNTs-TFN, (C) Ag-TFN, (D) $\mathrm{Al}_{2} \mathrm{O}_{3}-\mathrm{TFN}$, (E) $\mathrm{Fe}_{2} \mathrm{O}_{3}-\mathrm{TFN}$, (F) $\mathrm{TiO}_{2}-\mathrm{TFN}$. 
Chapter Seven - Thin Film Nanocomposite membranes modified with polydopamine coated metals/carbon-nanostructures for desalination applications.

\subsubsection{Atomic force microscope (AFM)}

The atomic force microscopy has demonstrated a wide range of application in the characterisation of the physical characteristics of surfaces. With many imaging modes, AFM is capable of imaging down to the sub-nanometre scale it builds up threedimensional topographies (Hilal et al., 2017). AFM tapping mode 3-D images illustrating the surface topography of TFC and TFN are presented in Figure 7.9. A clear "leaf-like folds" structure, linked to the PA layer formation, can be observed on the surface of TFC membrane, Figure 7.9A. Meanwhile, introducing the intermediate layer of PDA coated HNS, to form TFN membranes, has changed the structure, where a combination of "leaf-like" and "ridge-to valley" structure can be observed, Figure 7.9B-F. This could be due to the interruption of nanostructures on the growth of "leaflike" structure during the polymerisation reaction (Yin et al., 2016). Moreover, the surface roughness parameters have been improved upon the addition of HNS, compared to bare TFC membrane. This enhancement in TFN surface morphology smoothness is more likely to be attributed to the well distributed HNS intermediate layer. Indeed, this provides not only a robust mechanical support but also a smooth and homogenous platform for the subsequent IP process. All roughness parameters, root mean square roughness-Rms, root average arithmetic roughness- $\mathrm{R}_{\mathrm{a}}$, and ridge to valley distance$\mathrm{R}_{\max }$, are summarised in Table 7.1.

Table 7.1: Average surface roughness parameters of TFC and TFN membranes

\begin{tabular}{cccc} 
Membrane & $\mathrm{R}_{\mathrm{ms}}(\mathrm{nm})$ & $\mathrm{R}_{\mathrm{a}}(\mathrm{nm})$ & $\mathrm{R}_{\max }(\mathrm{nm})$ \\
\hline TFC & 33.689 & 26.792 & 241.07 \\
$f$-CNTs-TFN & 13.196 & 10.268 & 110.46 \\
$\mathrm{Al}_{2} \mathrm{O}_{3}$-TFN & 15.395 & 11.974 & 93.135 \\
$\mathrm{Fe}_{2} \mathrm{O}_{3}$-TFN & 21.662 & 16.783 & 194.53 \\
$\mathrm{TiO}_{2}$-TFN & 13.78 & 10.511 & 94.717 \\
$\mathrm{Ag}$-TFN & 20.303 & 15.414 & 151.58 \\
\hline
\end{tabular}


Chapter Seven - Thin Film Nanocomposite membranes modified with polydopamine coated metals/carbon-nanostructures for desalination applications.

A

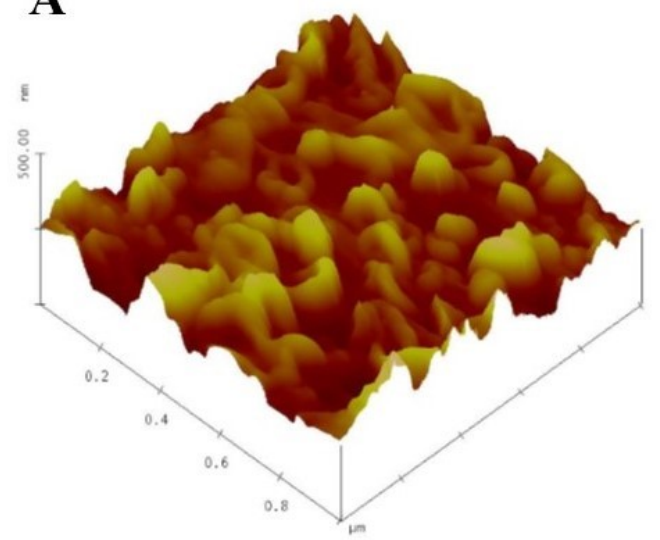

C

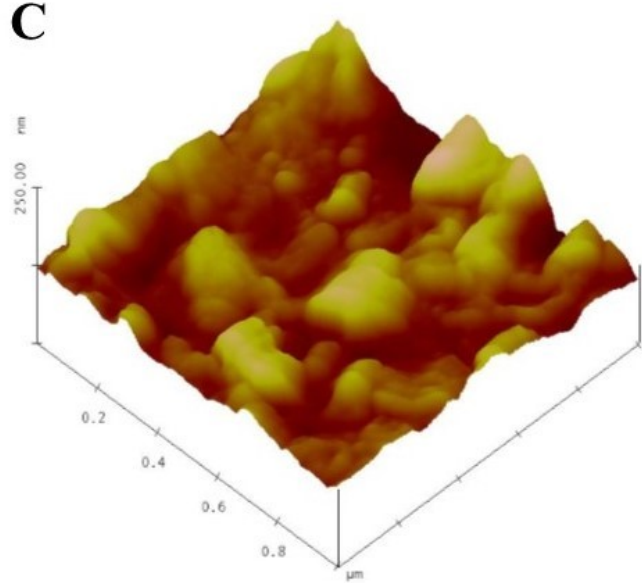

$\mathbf{E}$

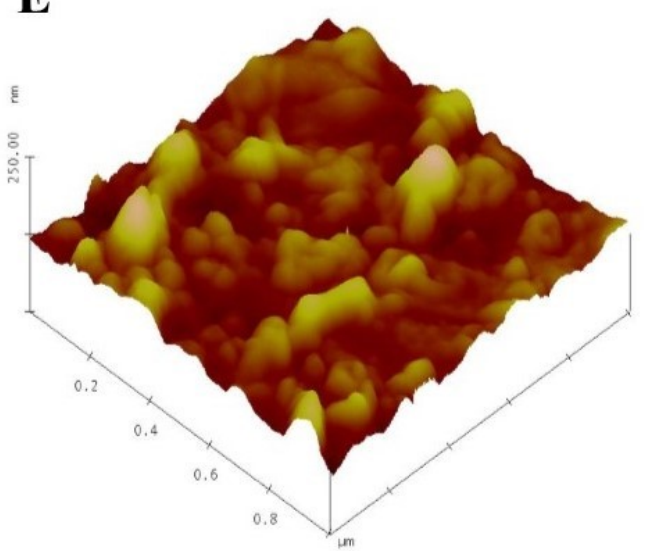

B

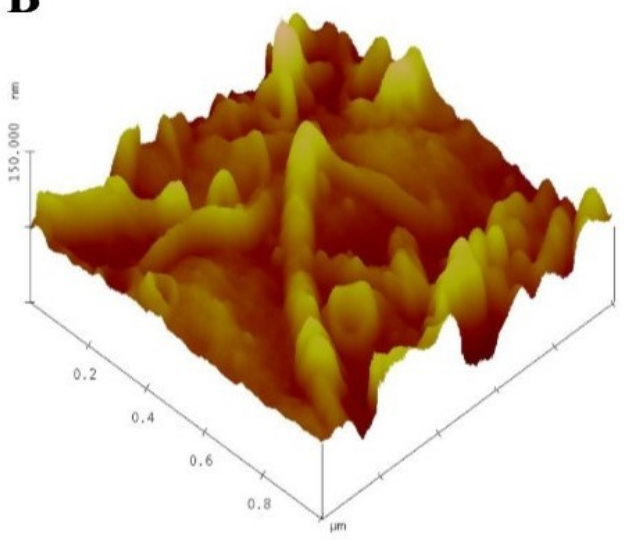

D

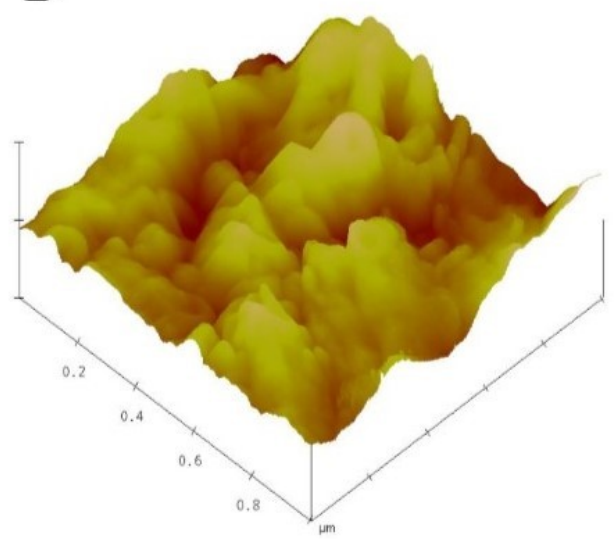

$\mathbf{F}$

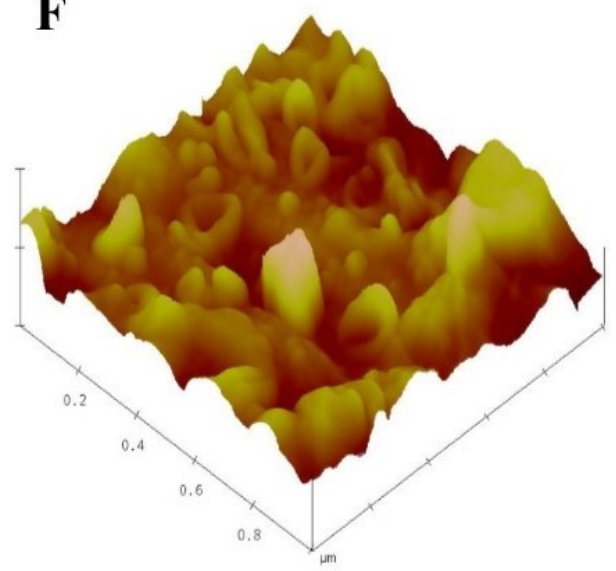

Figure 7.9: Three-dimensional-AFM images $(5 \mu \mathrm{m} \times 5 \mu \mathrm{m})$ of; (A) TFC, (B) f-CNTTFN, (C) $\mathrm{Al}_{2} \mathrm{O}_{3}-\mathrm{TFN}$, (D) $\mathrm{Fe}_{2} \mathrm{O}_{3}-\mathrm{TFN}$, (E) $\mathrm{TiO}_{2}-\mathrm{TFN}$ and (F) Ag-TFN. 
Chapter Seven - Thin Film Nanocomposite membranes modified with polydopamine coated metals/carbon-nanostructures for desalination applications.

\subsubsection{Static contact angle and hydrophilicity measurements}

Contact angle measurements were carried out to determine the role of PDA coated HNS incorporation on surface hydrophilicity/hydrophobicity of NF membranes. A noticeable decrease in the water contact angle (WCA) values was detected upon incorporating the nanostructures for all TFN membranes, as presented in Figure 7.10. The WCA of the bare TFC $\left(57.5^{\circ}\right)$ reported in this work was slightly lower than values $\sim\left(60^{\circ}-75^{\circ}\right)$ reported in the literature for a PA skin layer. In fact, disparate fabrication conditions (concentration of monomers, reaction time and support MF/UF characteristics) could result in some variation not only in the WCA values but also the overall characteristics of TFC membranes. The WCA of the f-CNTs-TFN membrane, prepared with PDA coated f-MWCNTs, declined to $54^{\circ}$ compared to that of TFC membrane. This decrease is suggested to be caused by the hydrophilic PDA and CNTs moieties in the TFN. In addition, the presence of M/MO NPs on the surface of CNTs further diminished the average WCA values of TFN membranes by $\sim 10^{\circ}$ approximately, which was found to be $46^{\circ}, 46.2^{\circ}, 43^{\circ}$ and $47.6^{\circ}$ for $\mathrm{Al}_{2} \mathrm{O}_{3}-\mathrm{TFN}, \mathrm{TiO}_{2}-$ TFN, $\mathrm{Fe}_{2} \mathrm{O}_{3}-\mathrm{TFN}$ and Ag-TFN membranes, respectively.

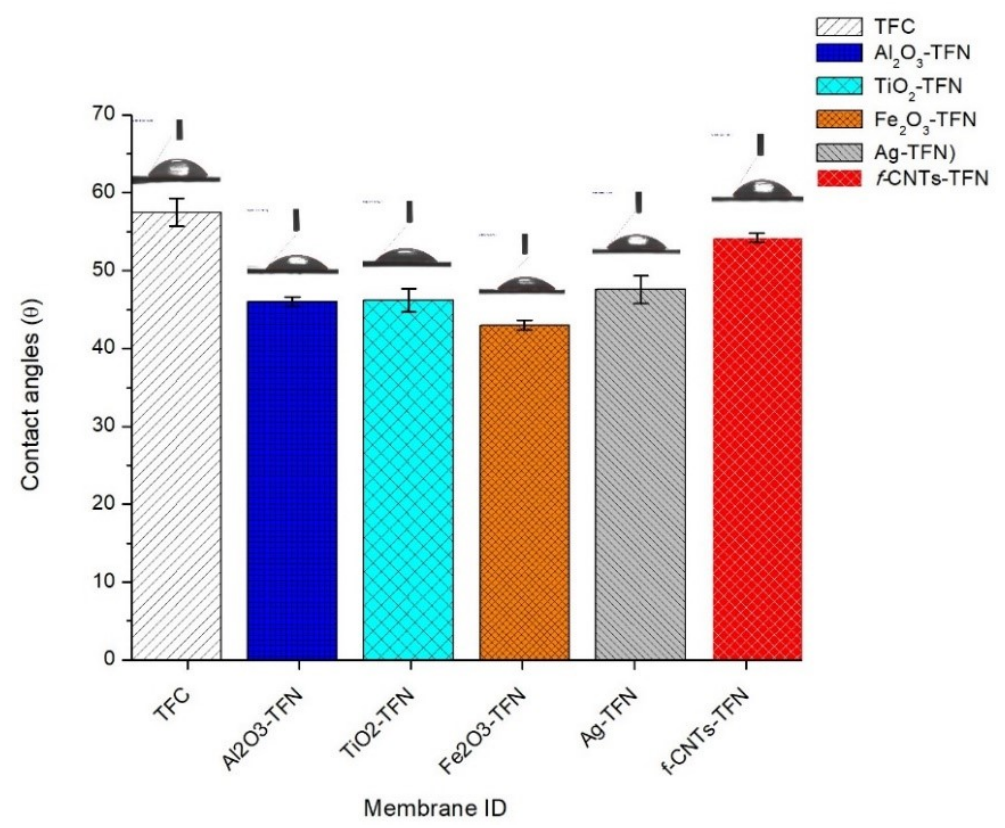

Figure 7.10: Static contact angle measurements of TFC and TFN membranes 
Chapter Seven - Thin Film Nanocomposite membranes modified with polydopamine coated metals/carbon-nanostructures for desalination applications.

\subsubsection{Performance evaluation of TFN}

\subsubsection{Monovalent and divalent salts}

The water permeation and separation characteristics for TFN membranes, synthesised with different types of $\mathrm{M} / \mathrm{MO}-\mathrm{MWCNTs}$ hybrid nanostructures under identical conditions $\left(2000 \mathrm{ppm}, \mathrm{pH} 6.8\right.$ and $\left.21^{\circ} \mathrm{C}\right)$, were compared with that of TFC membrane. A representative monovalent $(\mathrm{NaCl})$, divalent $\left(\mathrm{Na}_{2} \mathrm{SO}_{4}\right.$ and $\left.\mathrm{MgSO}_{4}\right)$, and a mixture of the three salts have been used for the separation's evaluation.

A clear enhancement was observed in the water permeability characteristic of all TFN, compared to that of the TFC membrane Figure 7.11. Under 0.1 MPa applied pressure, the pure water flux (PWF) of TFN membrane, made with an $f$-CNTs layer, was almost double $\left(10.5 \pm 0.2 \mathrm{l} / \mathrm{m}^{2} \cdot \mathrm{hr}\right)$ compared to that for the TFC membrane $(5.4 \pm$ $0.1 \mathrm{l} / \mathrm{m}^{2} \cdot \mathrm{hr}$ ). In addition, a further increase to $11.6 \pm 0.15 \mathrm{l} / \mathrm{m}^{2} \cdot \mathrm{hr}$ in PWF was observed with Ag-TFN membrane, while it had the lowest value $\left(9.6 \pm 0.1 \mathrm{l} / \mathrm{m}^{2} \cdot \mathrm{hr}\right)$ for $\mathrm{TiO}_{2-}$ TFN. This substantial flux improvement exhibited by TFN membranes is more likely to be caused by the imparted hydrophilic nature of PDA and HNS synergy on the PA skin layer, facilitating the solubilization and diffusion of $\mathrm{H}_{2} \mathrm{O}$ molecules through the thin film. However, it should be noted that a membrane's permeability is a complex matter, and cannot be attributed to the hydrophilic nature of the nanostructured interlayer only, as other factors like surface roughness, charges, and pore size/porosity may also contribute. The variation in PWF recorded was in the following order for $\mathrm{Al}_{2} \mathrm{O}_{3}-\mathrm{TFN}>\mathrm{Ag}-\mathrm{TFN}>\mathrm{f}-\mathrm{CNTs}-\mathrm{TFN}>\mathrm{Fe}_{2} \mathrm{O}_{3}-\mathrm{TFN}>\mathrm{TiO}_{2}-\mathrm{TFN}>\mathrm{TFC}$ membrane.

The flux decline during the single solute separation was in the order of $\mathrm{NaCl}>$ $\mathrm{MgSO}_{4}>\mathrm{Na}_{2} \mathrm{SO} 4$, respectively Figure 7.11A - C. While for the ternary salt filtration, the permeate flux decline was found to be somewhere in the middle of the identical mole fractions of each individual salt used in the filtration experiments, Figure 7.11D. 
Chapter Seven - Thin Film Nanocomposite membranes modified with polydopamine coated metals/carbon-nanostructures for desalination applications.
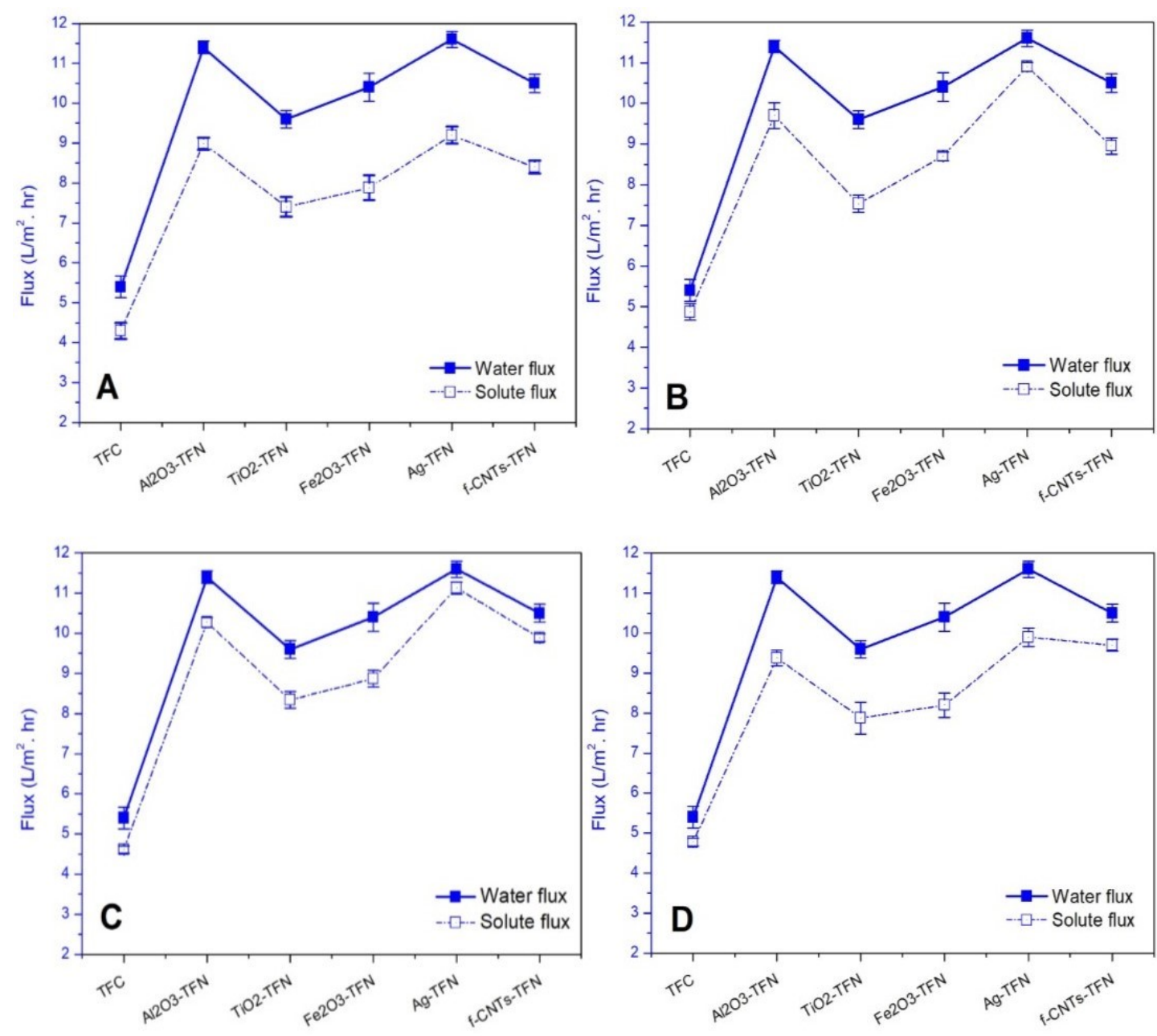

NF Membrane

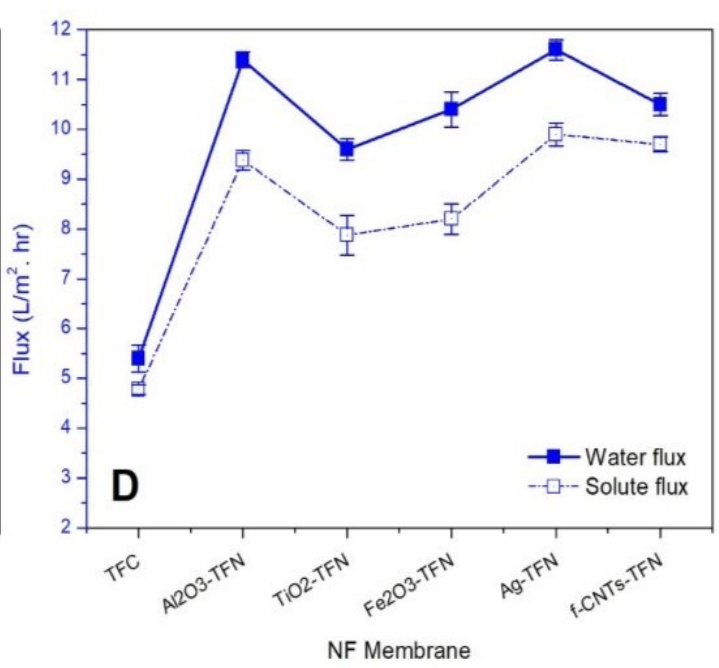

Figure 7.11: Pure water and solute permeation of NF membranes against; (A) NaCl, (B) $\mathrm{Na}_{2} \mathrm{SO}_{4},(\mathrm{C}) \mathrm{MgSO}_{4}$ and (D) ternary salt solution. $\left(2000 \mathrm{ppm}, 21^{\circ} \mathrm{C}, 1 \mathrm{MPa}\right)$

In regard of single salt separation, a negligible loss in monovalent salts rejection was observed for all TFN comparing to TFC, where all NF membranes rejection factors were ranging between $92.60 \%$ to $93.67 \%$, except that of Ag-TFN (91\%), Figure 7.12A. While, $99.44 \%$ and $97.15 \%$ rejection was obtained by TFC membrane for the divalent salts, $\mathrm{Na}_{2} \mathrm{SO}_{4}$ and $\mathrm{MgSO}_{4}$, respectively. Unsurprisingly, this was expected as typical negatively charged PA-TFC membranes have a higher repulsion to divalent sulfate anions $\left(\mathrm{SO}_{4}{ }^{-}\right)$than monovalent chloride anions $\left(\mathrm{Cl}^{-}\right)$due to Donnan-exclusion effects alongside the molecular sieving mechanism (Kim \& Deng, 2011). TFN membranes exhibited a higher potential for $\mathrm{SO}^{-}$anions separation than $\mathrm{Cl}^{-}$as well, but an explicit reduction in their divalent salts selectivity was noticed compared to TFC membrane, mainly for $\mathrm{Na}_{2} \mathrm{SO}_{4}$, Figure 7.12B. This could be attributed to the shift observed on the 
Chapter Seven - Thin Film Nanocomposite membranes modified with polydopamine coated metals/carbon-nanostructures for desalination applications.

zeta potential values of nanotubes after coating with positively charged $\mathrm{M} / \mathrm{MO}$ and PDA to form the HNS, Figure 7.5. Indeed, some positive charges have been rendered on the TFN surface if compared with the TFC, and this lowered the intrinsic negative charges of PA, as reported by (Lv et al., 2016). Du and Zhao indicated that selectivity of positively charged NF can be dissimilar to that of negatively charged NF membranes, wherein positively charged TFC membranes can have a higher rejection to $\mathrm{Cl}^{-}$than $\mathrm{SO}_{4}^{-}$(Du \& Zhao, 2004). Consequently, ionic transport and retention performance of NF membranes might reflect their charge characteristics. Therefore, in the present research, the zeta potential of the surface of TFN-NF membranes may have led to some discrepancy in the rejection behaviour of the NF membranes.
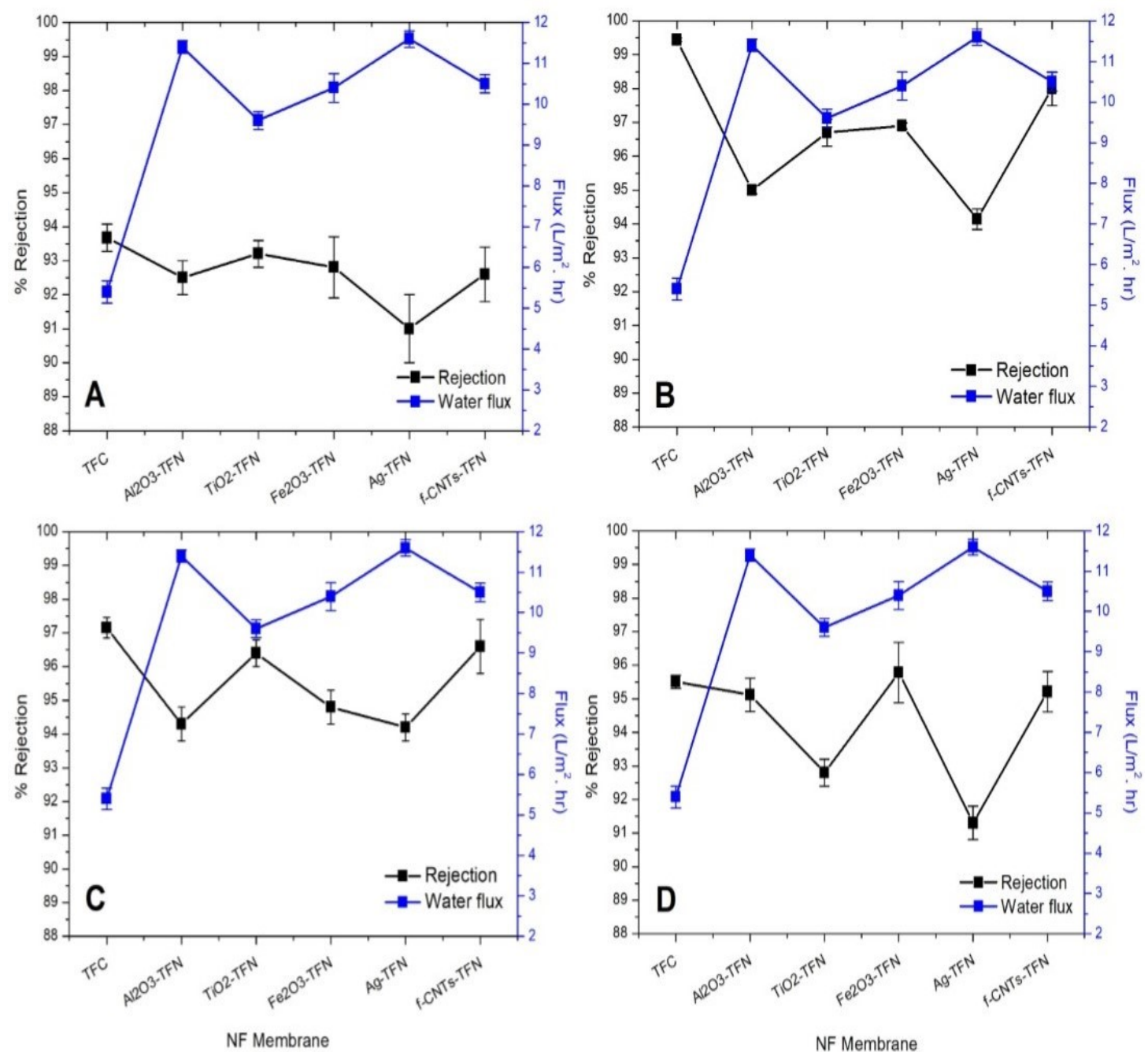

Figure 7.12: Pure water and solute rejection of NF membranes against; (A) $\mathrm{NaCl}$, (B) $\mathrm{Na}_{2} \mathrm{SO}_{4},(\mathrm{C}) \mathrm{MgSO}_{4}$ and (D) ternary salt solution. $\left(2000 \mathrm{ppm}, 25{ }^{\circ} \mathrm{C}, 1 \mathrm{MPa}\right)$ 
Chapter Seven - Thin Film Nanocomposite membranes modified with polydopamine coated metals/carbon-nanostructures for desalination applications.

The similar separation values indicate a successful construction of PA layer on the HNS intermediate layer without causing any defect in the skin layer of TFN. It should be noted that alignment of HNS interlayer on the PES substrate is crucial to facilitate the defects free PA layer construction process. Since their internal diameters are much higher than that of the selective PA skin layer, it is believed that any unaligned or/and uncovered nanotubes could act as channels for salts passage as well. In addition, it is critical to ensure a high stability in practical applications, through establishing a strong interfacial adhesion between the nanostructures layer and the PA on one side, and guarantee a firm interfacial adhesion with the PES substrate surface from the other side, so as to avoid any possible peeling of nanocomposite layer under cross-flow and highpressure conditions (Wu et al., 2016). This can be achieved through controlling the deposited HNS layer thickness, pore size and roughness of substrate membrane, monomer concentration, reaction time and cross-linking conditions. Any increase in the concentration of HNS above the limit used in this work $\left(0.0025 \mathrm{mg} / \mathrm{cm}^{2}\right)$ was found to cause a further increase in the HNS interlayer thickness, that can act as a separator between the PA and PES substrate rather than strengthening the nanostructure. Indeed, Figure 7.13 shows a membrane prepared with deposition of $0.01 \mathrm{mg} / \mathrm{cm}^{2} \mathrm{HNS}$. During the filtration experiments, the interlayer peeled off and could not survive the cross-flow conditions used (high pressure and flow rate).
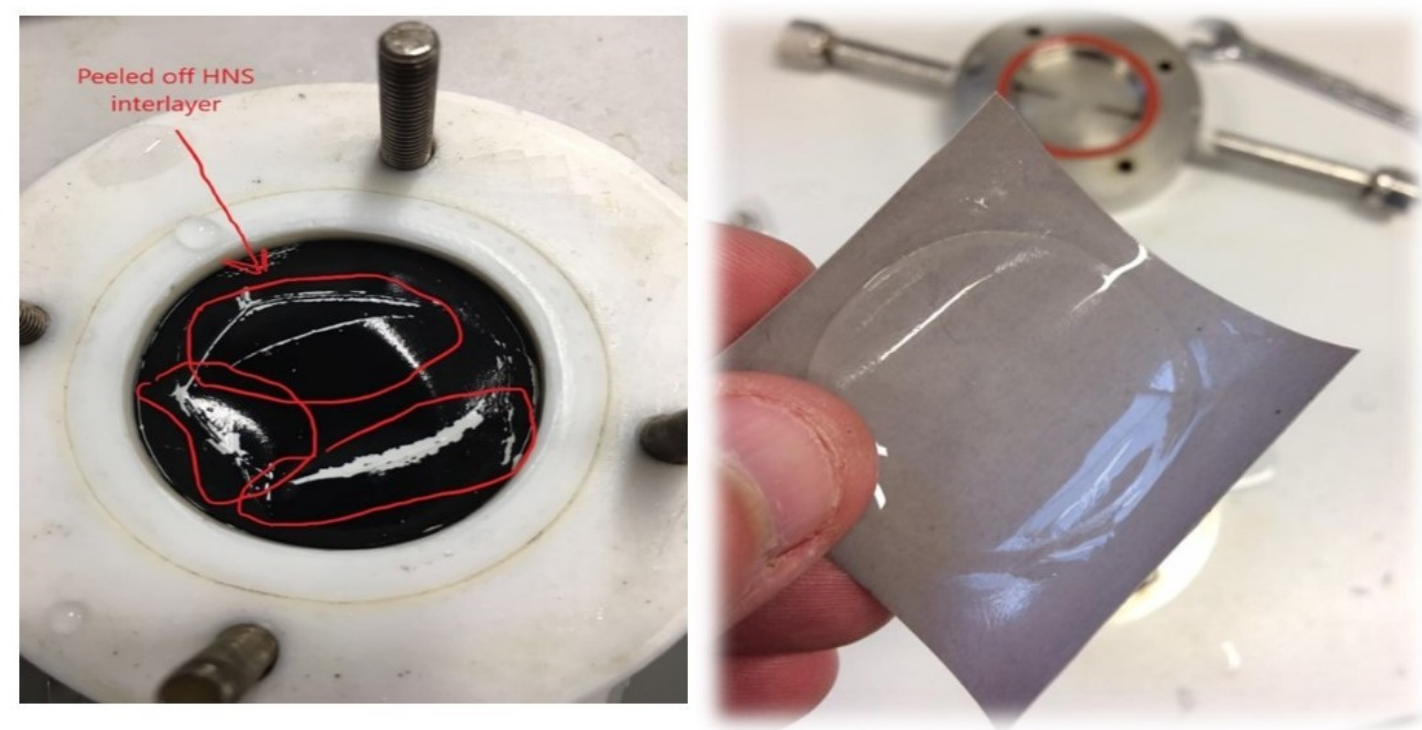

Figure 7.13: Peeling off the PA-HNS thin film layer under cross-flow conditions (Left), and TFN membrane prepared in this work (Right). 
Chapter Seven - Thin Film Nanocomposite membranes modified with polydopamine coated metals/carbon-nanostructures for desalination applications.

\subsubsection{Heavy metals}

Heavy metals are well known as an environmentally persistent contaminant having high toxicity and tendency to accumulate inside body tissues (Mohammad et al., 2004). To further assess the potential of fabricated TFN membranes in water treatment applications, a multi-component synthetic feed solution comprises of five heavy metal ions $\left(\mathrm{Mn}^{2+}, \mathrm{Ni}^{2+}, \mathrm{Pb}^{2+}, \mathrm{Cu}^{2+}\right.$, and $\left.\mathrm{Co}^{2+}\right)$, were passed through the membranes. $\mathrm{A}$ concentrated mixture of metals was used instead of a single solution to mimic serious wastewater that possibly would manifest lower potential removal than a single or a diluted solution (Qin et al., 2004). Prior to measurements, a calibration curve, as shown in Figure 7.14, was created for each individual metal and the results determined by AAS instrument (Figure 3.2).

As shown in Figure 7.15A, a noticeable augmentation in the pure water permeability characteristics of the TFN membranes was observed. This was attributed to enhancement in their surface hydrophilicity achieved after HNS interlayer deposition as discussed in Section 7.5.4. Following the feed solution filtration, the observed relative flux decline reached $7.8 \%, 13.7 \%, 11.1 \%, 12 \%$ and $12.3 \%$ for $\mathrm{Al}_{2} \mathrm{O}_{3}-\mathrm{TFN}$, Ag-TFN, f-CNTs-TFN, $\mathrm{Fe}_{2} \mathrm{O}_{3}$-TFN and $\mathrm{TiO}_{2}$-TFN membrane respectively, as compared with the TFC membrane $(28.6 \%)$. The pronounced reduction in the relative flux might be ascribed to the adsorption and cake layer formation of the metal hydroxides species on the surface of TFC and TFN membranes (Badawy et al., 2011). Meanwhile, the separation performance of all membranes tested was highly stable against the multi-component heavy metal solution. Noticeably, manganese retention was higher than other metals (99.5 - 100\%) (Figure 7.15B). This could be attributed to the differences in the diffusion coefficients of cations in water that inversely reflects on their retention sequence (Al-Rashdi et al., 2013). However, membranes rejection effectiveness was minimum $(92.5 \%-95.2 \%)$ against $\mathrm{Ni}^{2+}$, as shown in Figure 7.15C. Other retention coefficients were found to be $(98.3 \%-100 \%),(96.5 \%-100 \%)$ and (92.4\% - 95.6\%) for $\mathrm{Pb}^{2+}, \mathrm{Cu}^{2+}$ and $\mathrm{Co}^{2+}$, respectively (Figure 7.15D - Figure 7.15F). 
Chapter Seven - Thin Film Nanocomposite membranes modified with polydopamine coated metals/carbon-nanostructures for desalination applications.

Ni 232.00

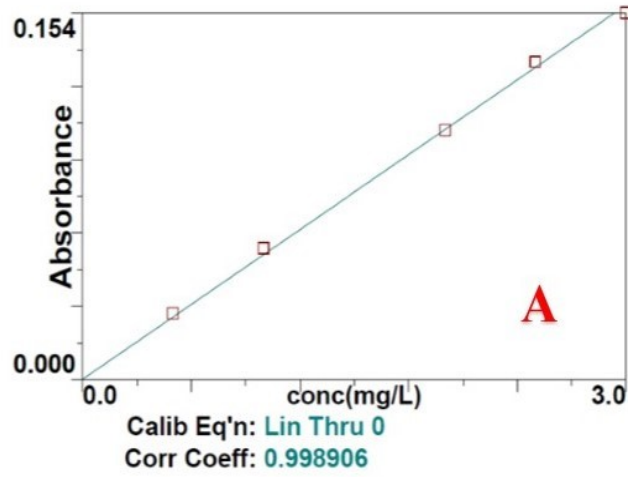

Cu 324.75

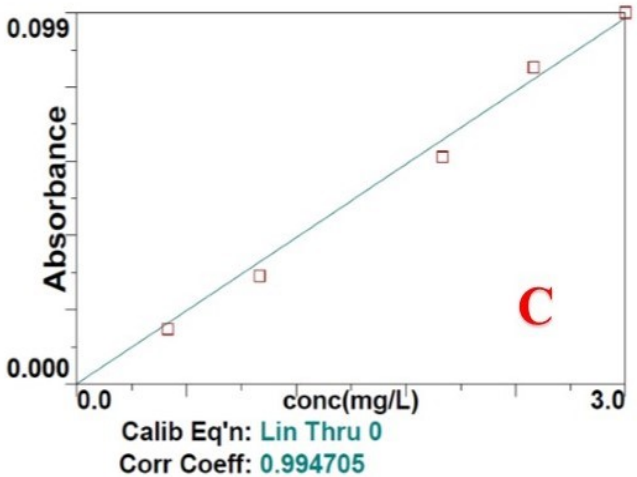

Mn 279.48

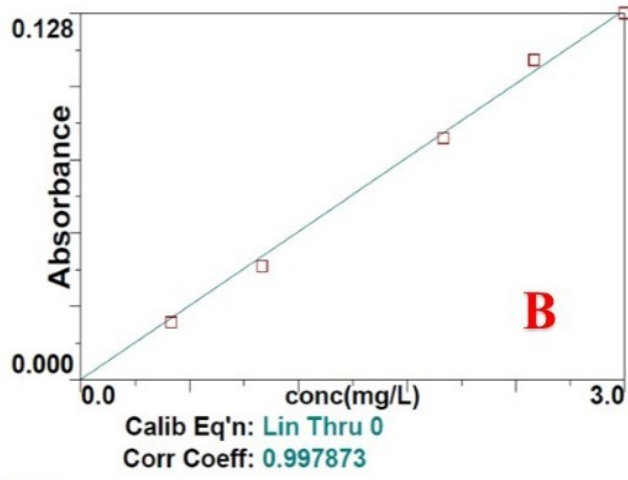

Co 240.73

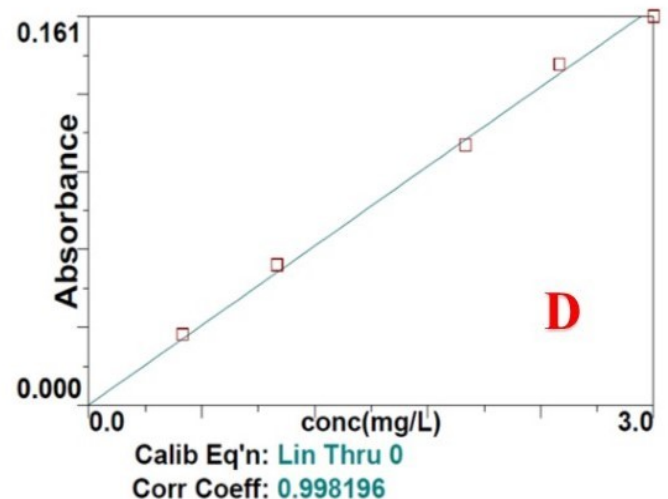

$\mathrm{Pb} 283.31$

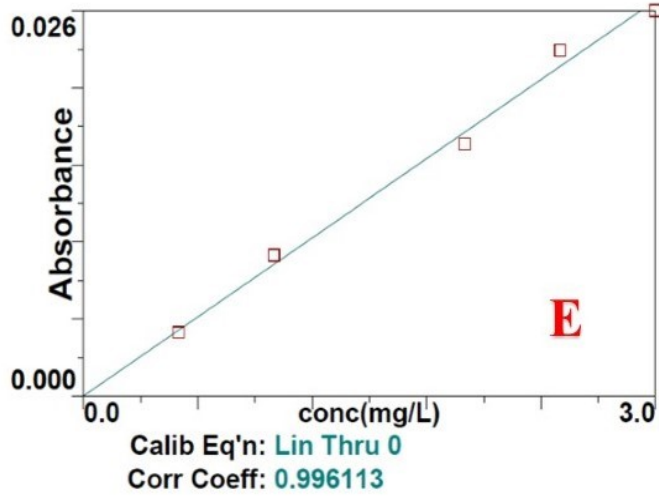

Figure 7.14: Calibration curve of heavy metal solutions determined by AAS, (A) Nickel, (B) Manganese, (C) Copper, (D) Cobalt, and (E) Lead. 
Chapter Seven - Thin Film Nanocomposite membranes modified with polydopamine coated metals/carbon-nanostructures for desalination applications.

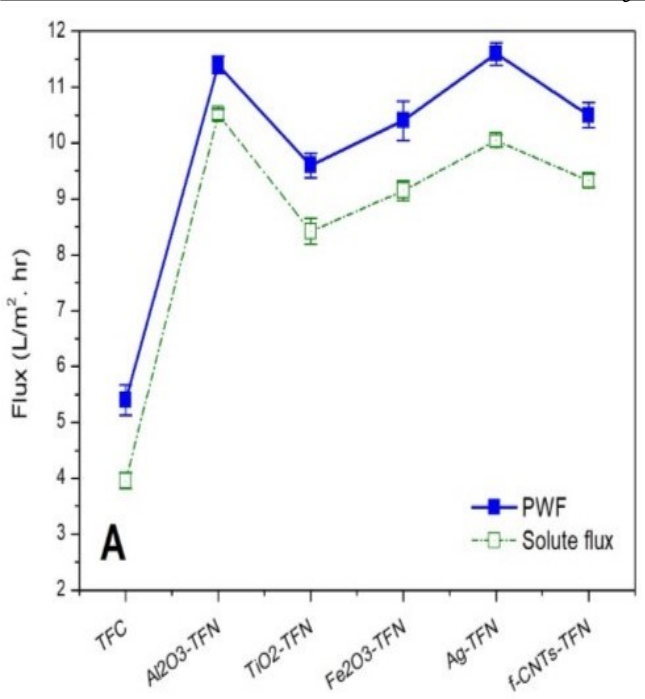

NF Membrane

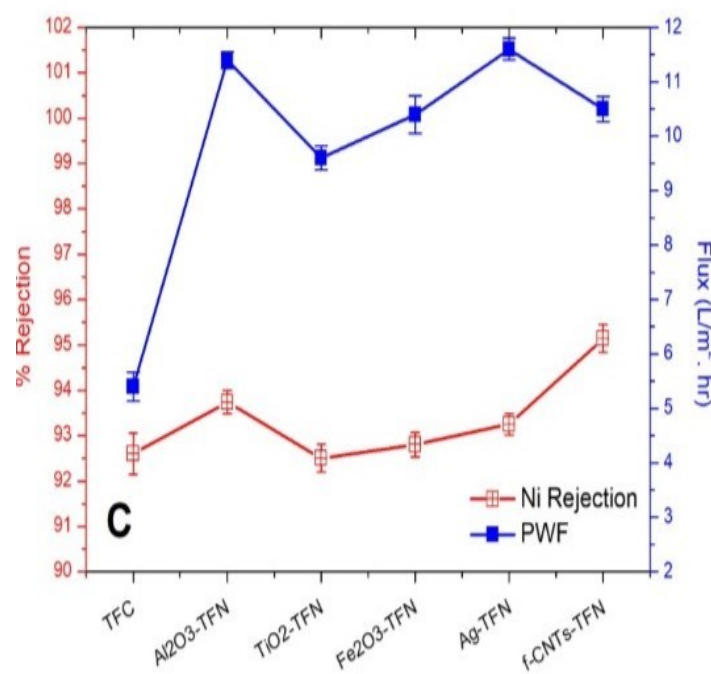

NF Membrane

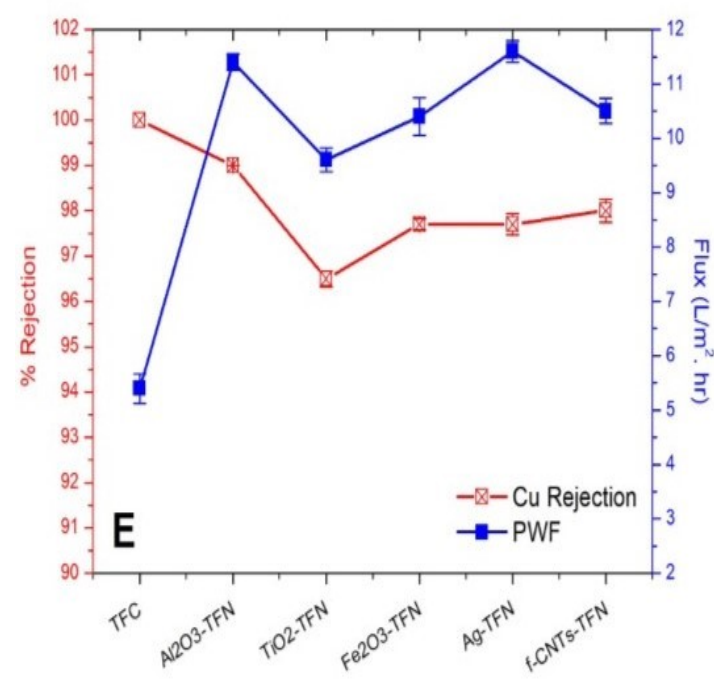

NF Membrane

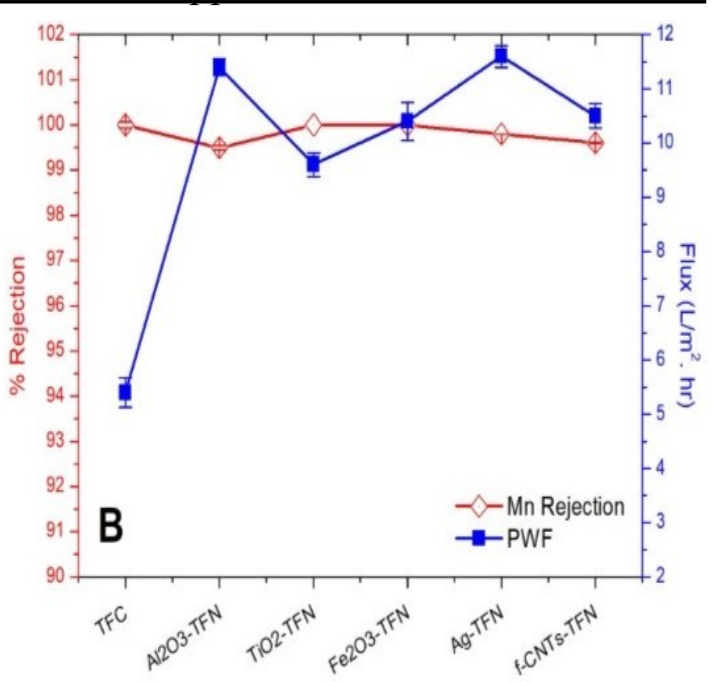

NF Membrane

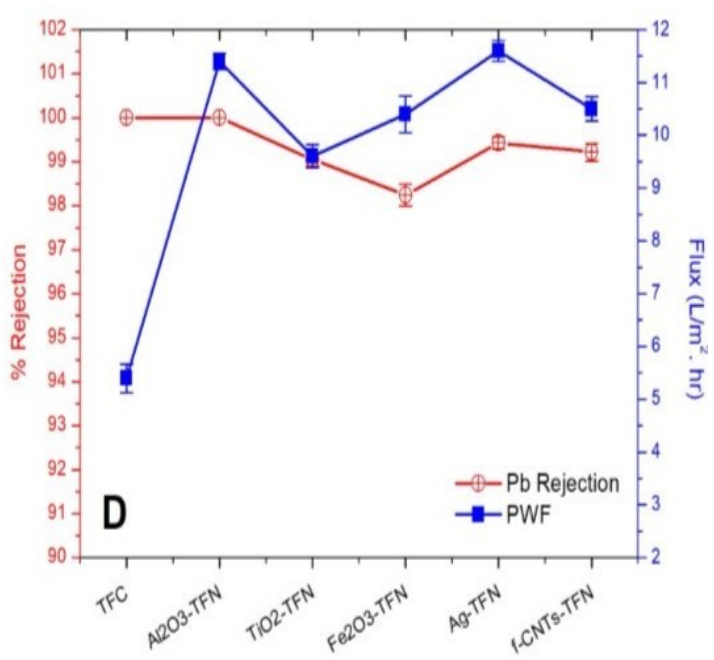

NF Membrane

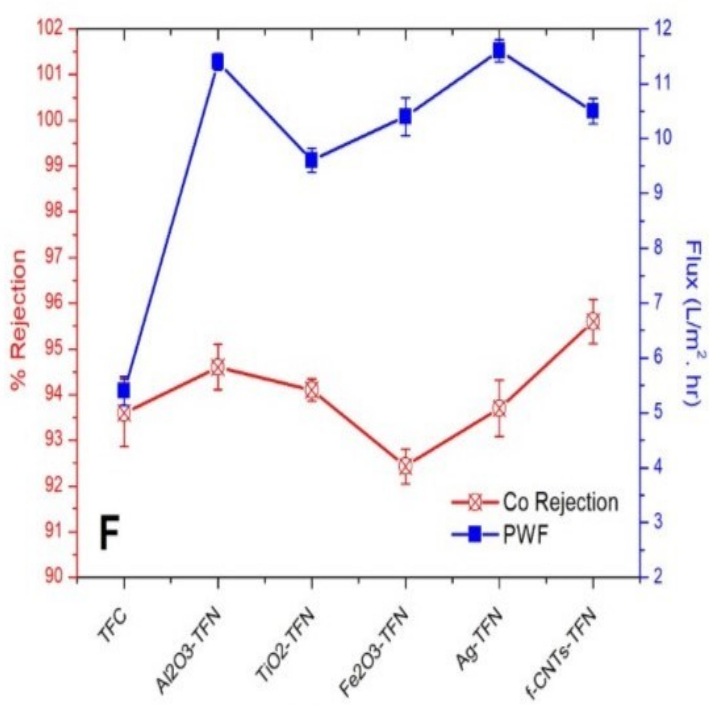

NF membrane

Figure 7.15: PWF vs solute flux of metal mixture (A), retention vs PWF for (B) $\mathrm{Mn}^{2+}$, (C) $\mathrm{Ni}^{2+}$, (D) $\mathrm{Pb}^{2+}$, (E) $\mathrm{Cu}^{2+}$, and (F) $\mathrm{Co}^{2+}$. (feed concentration= $250 \mathrm{ppm}, 2{ }^{\circ} \mathrm{C}$, $\mathrm{pH}=5,1 \mathrm{MPa})$ 
Chapter Seven - Thin Film Nanocomposite membranes modified with polydopamine coated metals/carbon-nanostructures for desalination applications.

\subsection{Conclusions}

Novel TFN membranes for desalination applications were developed in this work, based on the fabrication and design of novel HNS. M/MO-MWCNTs modulated by calcination were wrapped by a thin layer of polydopamine. The felicitous muscle inspired chemical packaging on the surface of HNS was confirmed by FTIR while the extent of PDA grafting on f-MWCNTs and other HNS was quantified by thermogravimetric analysis, and ranged between 34-45 wt.\%. Zeta potential measurements were conducted to investigate the impact of PDA layer on the HNS zeta potential, where coating the nanocomposites with PDA caused charge reversal, especially in an acidic environment. The PDA coated HNS were assessed in terms of performance of the fabricated TFN membranes when introduced as an intermediate layer to revise their surface characteristics. Their surface and cross-section morphologies were depicted by SEM imaging. Furthermore, TFN manifested smoother and more hydrophilic surfaces if compared with TFC membrane. Also, nearly double the PWF was obtained from TFN membrane compared to that of TFC membrane, without affecting the permeability/selectivity trade-off relationship. The variation in PWF recorded was in the following order for $\mathrm{Al}_{2} \mathrm{O}_{3}-\mathrm{TFN}\left(11.4 \pm 0.1 \mathrm{l} / \mathrm{m}^{2} \cdot \mathrm{hr}\right)>\mathrm{Ag}$ TFN $\left(11.6 \pm 0.15 \mathrm{l} / \mathrm{m}^{2}\right)>$ f-CNTs-TFN $\left(10.5 \pm 0.2 \mathrm{l} / \mathrm{m}^{2} \cdot \mathrm{hr}\right)>\mathrm{Fe}_{2} \mathrm{O}_{3}-\mathrm{TFN}(10.2 \pm 0.2$ $\left.1 / \mathrm{m}^{2} \cdot \mathrm{hr}\right)>\mathrm{TiO}_{2}-\mathrm{TFN}\left(9.6 \pm 0.1 \mathrm{l} / \mathrm{m}^{2} \cdot \mathrm{hr}\right)>\mathrm{TFC}$ membrane $\left(5.4 \pm 0.1 \mathrm{l} / \mathrm{m}^{2} \cdot \mathrm{hr}\right)$. In regard of single salt separation, a negligible loss in monovalent salts rejection was observed for all TFN comparing to TFC, where all NF membranes rejection factors were ranging between $92.60 \%$ to $93.67 \%$, except that of $\mathrm{Ag}$-TFN (91\%), while a little reduction in their divalent salts selectivity was noticed. Finally, it should be noted that alignment of HNS interlayer on the PES substrate is crucial to facilitate the defects free PA layer construction process. The effectiveness of TFN membranes in remediation of wastewater comprising a mixture of heavy metals was also investigated. The results manifested that the TFN membranes have significantly reclaimed wastewaters with an initial concentration of $250 \mathrm{ppm}$ and reduced the metal ion concentration by $>92$ to $100 \%$ removal based on the metal used. Besides that, a noticeable enhancement in the fouling resistance of the TFN membranes was achieved through the surface modification. This implied that the TFN fabricated in this work are capable to reclaim a good quality water for further use, along with better antifouling and permeation characteristics. 


\section{Chapter Eight}

\section{Conclusions and Recommendations}

\subsection{Conclusions}

In the first instance, the present work investigated, step by step, the design and fabrication of nanocomposite membranes to overcome the limitations of nanocomposite applications presented by the weak interfacial interactions at the interface between the polymeric matrix and NMs.

In order to provide better insights into the role of PES concentration on the morphology and surface characteristics, a comprehensive characterisation study was conducted. Asymmetric UF membranes with versatile morphologies and characteristics have been produced via the classical non-induced phase separation. As a result of variation in the viscosity of casting solutions, significant differences in the PWF, PS and PSD, cross-section morphology and mechanical strength were observed, while this influence was insignificant for roughness parameters, water contact angle and surface charges of the membranes. A comparison was also made to provide valuable insight into details of organic molecules-membrane interactions to better understand the role of membranes surface properties when challenged with the natural organic matter, polysaccharides and proteins. Although the large variation in membranes surface properties, the differences in their rejection coefficients were not significant, as the different MWCO characteristics obtained are compromised by different fouling mechanisms. In addition, it should be noted that the influence of surface roughness parameters, hydrophilicity and surface charge cannot be considered in the comparison since these measurements were similar for all membranes. When the fouling studies were considered, the relative flux decline was negligible for the low MWCO 
membranes, PES22 and PES20, within the short filtration duration of 120 min used in this research. In contrast, extremely low relative flux patterns were observed in the filtration with the $100 \mathrm{kDa}$ membrane (PES16). Adsorption effects were small for the low cut-off PES22 (6 kDa) and PES20 (10 kDa) membranes with all organic models used. While they were significant for fouling at the PES18 (35 kDa) membrane. In contrast, the larger pore (PES16) membranes experienced an instantaneous adsorption resulting in a considerable relative flux decline, mainly in acidic conditions and highly concentrated feed solutions. Finally, although NaAlg manifested higher relative flux decline, it was mostly recoverable compared to HA and BSA filtration, which showed significant irreversible flux, mainly for the $100 \mathrm{kDa}$ membranes.

Since the theme of this research study focuses on polymeric membranes modification by means of nanoscale materials, novel hybrid nanostructures comprise $\mathrm{M} / \mathrm{MO}$-carbon nanotubes have been successfully fabricated via wet chemistry technique without reducing agents or any further chemicals. Two sets were made depending on the route employed to deposit the $\mathrm{M} / \mathrm{MO}$ on the surface of nanotubes. This was in turn modulated either by calcination in a high-temperature tube furnace or microwave irradiation. This thesis was the first study introduces the microwave irradiation in the application of nanocomposite membranes. Even though the quantitative and qualitative analysis confirmed the success of both methods, the microwave treatment was much faster route adopted during the fabrication, where only less than $4 \mathrm{hr}$ is needed to get the final sample ready. Results obtained confirmed the successful functionalization of MWCNTs with carboxylic groups without causing any serious damage/defects to the nanotube surface, while a series of characterisation means assessed the stability, surface structure, thermal, electrical properties, composition and oxidation state of the samples. It should be noted here that many characterisation tools were used in this work since some tools were unable to detect the low loading level of $\mathrm{M} / \mathrm{MO}$ on the surface of nanotubes and the small size nanoparticles (e.g., XRD). Therefore, more instruments have been used to provide a comprehensive characterisation of the synthesised HNS and to confirm the overall properties.

The microwave-modulated HNS were employed to tailor the membranes functions into a specific application. The two concentration levels of Ag /CNTs, named as10HNS and 20HNS, are incorporated within the PES polymeric matrix (bulk modification) at different loading levels to probe the role of $\mathrm{Ag}$ size and concentration on the biocidal 
characteristics of nanocomposite membranes. Based on the design and fabrication of this facile, rapid and scalable biocidal HNS, a novel antibacterial membrane material was developed in this work for engineered nanocomposite membrane applications, which is effective against both Gram-positive and Gram-negative bacteria, as confirmed by antibacterial tests using the representative species E. coli and $S$. aureus. Also, characterisation techniques have clearly identified the significant influence that $\mathrm{Ag} / \mathrm{MWCNTs}$ ratio has on the structure and performance of HNS, which in turn impacted on the fabricated nanocomposite membrane structure and process properties to different degrees. Meanwhile, the content of HNS within a PES membrane matrix was found to have little influence on the surface pore size and pore size distribution. However, the addition of the HNS to form a nanocomposite membrane had a significant impact on hydrophilicity measurement, pure water flux, surface zeta potential, and bactericidal performance of the nanocomposites. Moreover, antimicrobial tests suggest $\mathrm{Ag}^{+}$release was the dominant mechanisms against both bacterial species as there was no direct contact between the biocide material and microorganisms. This may be attributed to the fact that the HNS was immobilised within the polymeric chains close to the top surface of the nanocomposites. This observation can have great influence on the stability of the HNS inside the nanocomposites and their long-term antibacterial activity through providing a controlled silver ions release rather than a rapid depletion. Also, results have evidenced that lower Ag/MWCNTs ratio has manifested higher biocidal effects due to the higher number of silver atoms presented at the interface (higher surface area/volume ratio). This will indeed reduce the environmental and social cost of the final nanocomposite applications. In conclusion, such biocidal HNS and their incorporation into nanocomposite membranes have great potential in the design of low fouling self-cleaning membranes with a high degree of performance durability and commercial scalability.

Enhancing the interfacial interactions between organic polymers and ENMs is essential in order to establish highly dependable nanocomposite materials as the next generation of membranes. This thesis has successfully presented for the first time, the application of HNS into TFN membrane applications. Four M/MO-based HNS $\left(\mathrm{Fe}_{2} \mathrm{O}_{3} \mathrm{CNTs}\right.$, $\mathrm{TiO}_{2} \mathrm{CNTs}, \mathrm{Al}_{2} \mathrm{O}_{3} \mathrm{CNTs}$ and $\left.\mathrm{AgCNTs}\right)$, modulated by calcination at hightemperature tube furnace, were employed for developing novel TFN membranes for desalination applications. These HNS were coated with a thin layer of PDA then 
assessed in terms of performance of the fabricated TFN membranes when introduced as an intermediate layer to revise their surface characteristics. Nearly double the PWF was obtained from TFN membrane compared to that of TFC membrane, without affecting the permeability/selectivity trade-off relationship. In addition, higher than $90 \%$ and $94 \%$ removals were achieved for monovalent and divalent salts, respectively. Indicating that NF membranes have been fabricated are capable of treating various salts at a comparable level. Nevertheless, the experimental results of TFN membranes manifested great retention for relatively all metal ions, and were comparable to that of TFC membrane but with higher water permeation characteristics. Indeed, TFN could offer a promising potential for the applications of wastewater reclamation for further use. On the other hand, the design of defect-free and mechanically stable PA layer on the HNS supported PES substrate was found to be contingent on the interlayer thickness. Undoubtedly, this thesis has demonstrated a great potential to use high loading level of HNS within the PA layer without defects. Also, the novel methodology used was able to overcome the limitation of other conventional TFN fabrication techniques. The theoretical amount of NMs used in this methodology corresponds to the actual amount deposited on the surface, unlike other techniques which wastes a higher amount only to incorporate a small quantity with uneven distribution. Indeed, this will reduce the environmental and economic costs of TFN membrane application. Additionally, with the powerful catechol groups of the mussel-inspired PDA layer, the great potential to link these HNS with the PA chain has added further stability and compatibility to the TFN under the cross flow conditions. In summary, the clear enhancement in the surface characteristics of TFN membranes attributed to the stable hydrophilic intermediate layer introduced and masked by the PA.

\subsection{Recommendations.}

Recommendations for future work have been suggested as follows;

1. In this study, Ag-based TFN membranes have been fabricated and their performance evaluation was carried in desalination and wastewater applications. Further evaluation regarding their antibacteriostaic potential is 
essential to be carried against many biological species, e.g., E. coli, S. aureus, Pseudomonas aeruginosa and algal bloom. This will confirm the multifunctionality of the Ag-based TFN membranes to treat various contaminated waters. Especially, the antibacterial HNS are presented very close to the surface and expected to manifest a greater antimicrobial activity.

2. Mechanical tests for virgin and fouled control and modified membrane would be worth trying in order to provide a clear evaluation of their mechanical robustness against various cleaning and process conditions.

3. Further research is required before commercialisation to ensure that the benefits of ENM-membrane nanocomposites outweigh their fabrication and environmental cost. This can be carried by performing prolonged operations under real conditions to measure their consistency and reliability.

4. Treating a real seawater by employing the nanocomposite membranes as a pretreatment step for the later TFN membranes, then compare the results with a similar unit consist of a commercial UF/ tide NF membrane. This can give a better performance evaluation for the hand-made membranes in terms of selectivity against various salts, antifouling properties and reliability of measurements comparing to the commercial membranes.

5. Evaluation of the adsorption capacity of the fabricated HNS in wastewater treatment applications and compare it with M/MO NPs and MWCNTs individually. This can provide a comparison data for the three materials to be used as adsorbents.

6. Determination of silver ions releases over a prolonged operation time to assess the reliability of the held antimicrobial activity of nanocomposites and TFN membranes.

7. To evaluate the ecological issues of use, by measuring the potential release of ENMs and their fate at the end of their service life. Also, to determine whether they have certain affinity to form complexes with other components in the discharge. Where these issues could raise the entire cost of any process.

8. Determining new routes to modify the surface characteristics of NMs and/or other HNS to for better stability and even distribution inside the nanocomposite membranes. 
9. For NMs surface modification/functionalisation, a focus should be made on employing environment-friendly and natural compounds that have minimal or no toxicity on the ecosystem (e.g., tannic acid). This will reduce the environmental cost.

10. Intensive research should be carried to provide new insights for surface modification of membrane applications in order to impart the characteristics of nanoscale materials close to the membrane-solution interface.

11. Searching for new routes to robustly tether 0D NPs on various $2 \mathrm{D}$ and $3 \mathrm{D}$ nanoscale materials to avoid using reducing agents and costly equipment.

12. More efforts should be devoted to the use of new exotic nanomaterials for novel nanocomposite membranes fabrication.

13. More attention should be paid to the use of environmental-friendly NMs, e.g., cellulose materials in order to obviate the environmental concerns associated with M/MO NPs.

14. Some literature reported that PDA poses bacteriostatic activity, so testing the toxicity of PDA-AgCNTs vs AgCNTs by zone of inhibition test could be worthwhile to compare between the samples.

15. AFM measurements can be used to measure the adhesion force between the microbial species and the Ag-based membrane. This may provide indicative data about the ability of the membrane to resist the biofilm. 


\section{References}

Abdul Khalil, H. P., Davoudpour, Y., Islam, M. N., Mustapha, A., Sudesh, K., Dungani, R., \& Jawaid, M. (2014). Production and modification of nanofibrillated cellulose using various mechanical processes: a review. Carbohydrate Polymers, 99, 649-665.

Abdul Khalil, H. P. S., Bhat, A. H., \& Ireana Yusra, A. F. (2012). Green composites from sustainable cellulose nanofibrils: A review. Carbohydrate Polymers, 87(2), 963-979.

Abu Seman, M. N. (2010). Development of antifouling properties for nanofiltration membranes. (P.hD.), University of Nottingham.

Acharya, V., Prabha, C. R., \& Narayanamurthy, C. (2004). Synthesis of metal incorporated low molecular weight polyurethanes from novel aromatic diols, their characterization and bactericidal properties. Biomaterials, 25(19), 45554562 .

Ahamed, M., Alsalhi, M. S., \& Siddiqui, M. K. (2010). Silver nanoparticle applications and human health. Clinica Chimica Acta, 411(23-24), 1841-1848.

Akar, N., Asar, B., Dizge, N., \& Koyuncu, I. (2013). Investigation of characterization and biofouling properties of PES membrane containing selenium and copper nanoparticles. Journal of Membrane Science, 437, 216-226.

Akhavan, O., \& Ghaderi, E. (2010). Toxicity of graphene and graphene oxide nanowalls against bacteria. ACS nano, 4(10), 5731-5736.

Al-Amoudi, A., \& Lovitt, R. W. (2007). Fouling strategies and the cleaning system of NF membranes and factors affecting cleaning efficiency. Journal of Membrane Science, 303(1), 4-28.

Al-Malek, S. (2012). Synthesis and characterization of polyethersulfone membrane using different additives. (Ph.D), Swansea University.

Al-Rashdi, B., Johnson, D., \& Hilal, N. (2013). Removal of heavy metal ions by nanofiltration. Desalination, 315, 2-17.

Alam, J., Dass, L. A., Ghasemi, M., \& Alhoshan, M. (2013). Synthesis and optimization of PES-Fe3O4mixed matrix nanocomposite membrane: Application studies in water purification. Polymer Composites, 34(11), 1870-1877.

Alimohammadi, F., Gashti, M. P., Shamei, A., \& Kiumarsi, A. (2012). Deposition of silver nanoparticles on carbon nanotube by chemical reduction method: Evaluation of surface, thermal and optical properties. Superlattices and Microstructures, 52(1), 50-62.

Amais, R. S., Ribeiro, J. S., Segatelli, M. G., Yoshida, I. V., Luccas, P. O., \& Tarley, C. R. (2007). Assessment of nanocomposite alumina supported on multi-wall carbon nanotubes as sorbent for on-line nickel preconcentration in water samples. Separation and Purification Technology, 58(1), 122-128.

Ang, W. L., Mohammad, A. W., Hilal, N., \& Leo, C. P. (2015). A review on the applicability of integrated/hybrid membrane processes in water treatment and desalination plants. Desalination, 363(Supplement C), 2-18. 
Antony, A., Low, J. H., Gray, S., Childress, A. E., Le-Clech, P., \& Leslie, G. (2011). Scale formation and control in high pressure membrane water treatment systems: a review. Journal of Membrane Science, 383(1), 1-16.

Aptel, P., \& Buckley, C. A. (1996). Categories of membrane operations. Chapter in Water Treatment Membrane Processes, McGraw Hill.

Asatekin, A., Menniti, A., Kang, S., Elimelech, M., Morgenroth, E., \& Mayes, A. M. (2006). Antifouling nanofiltration membranes for membrane bioreactors from self-assembling graft copolymers. Journal of Membrane Science, 285(1-2), 8189.

Badawy, S., Shokry, S., Ismail, A., \& Zeiada, M. (2011). Study on the removal of iron (III) and chromium (III) from aqueous streams using inorganic nanofiltration membrane. Australian Journal of Basic and Applied Sciences, 5(11), 236-243.

Bagchi, B., Dey, S., Bhandary, S., Das, S., Bhattacharya, A., Basu, R., \& Nandy, P. (2012). Antimicrobial efficacy and biocompatibility study of copper nanoparticle adsorbed mullite aggregates. Materials Science and Engineering: C, 32(7), 1897-1905.

Bai, H., Wang, X., Zhou, Y., \& Zhang, L. (2012). Preparation and characterization of poly(vinylidene fluoride) composite membranes blended with nano-crystalline cellulose. Progress in Natural Science: Materials International, 22(3), 250-257.

Bai, H., Zan, X., Zhang, L., \& Sun, D. D. (2015). Multi-functional CNT/ZnO/TiO2 nanocomposite membrane for concurrent filtration and photocatalytic degradation. Separation and Purification Technology, 156, 922-930.

Baker, R. W. (2004). Overview of membrane science and technology. Membrane Technology and Applications, Second Edition.

Balasubramanian, K., \& Burghard, M. (2005). Chemically functionalized carbon nanotubes. Small, 1(2), 180-192.

Balta, S., Sotto, A., Luis, P., Benea, L., Van der Bruggen, B., \& Kim, J. (2012). A new outlook on membrane enhancement with nanoparticles: The alternative of $\mathrm{ZnO}$. Journal of Membrane Science, 389, 155-161.

Baro, M., Nayak, P., Baby, T. T., \& Ramaprabhu, S. (2013). Green approach for the large-scale synthesis of metal/metal oxide nanoparticle decorated multiwalled carbon nanotubes. Journal of Materials Chemistry A, 1(3), 482-486.

Baroña, G. N. B., Lim, J., Choi, M., \& Jung, B. (2013). Interfacial polymerization of polyamide-aluminosilicate SWNT nanocomposite membranes for reverse osmosis. Desalination, 325, 138-147.

Baughman, R. H., Zakhidov, A. A., \& de Heer, W. A. (2002). Carbon nanotubes--the route toward applications. Science, 297, 787-792.

Becht, N. O. (2008). A study of charge and hydrodynamic effects in protein ultrafiltration. (Ph.D), Loughborough University.

Bergamasco, R., da Silva, F. V., Arakawa, F. S., Yamaguchi, N. U., Reis, M. H. M., Tavares, C. J., . . . Tavares, C. R. G. (2011). Drinking water treatment in a gravimetric flow system with $\mathrm{TiO} 2$ coated membranes. Chemical Engineering Journal, 174(1), 102-109. 
Bernhardt, E. S., Colman, B. P., Hochella, M. F., Cardinale, B. J., Nisbet, R. M., Richardson, C. J., \& Yin, L. (2010). An ecological perspective on nanomaterial impacts in the environment. Journal of Environmental Quality, 39(6), 19541965.

Blanco, J.-F., Sublet, J., Nguyen, Q. T., \& Schaetzel, P. (2006). Formation and morphology studies of different polysulfones-based membranes made by wet phase inversion process. Journal of Membrane Science, 283(1), 27-37.

Bourlinos, A. B., Gournis, D., Petridis, D., Szabó, T., Szeri, A., \& Dékány, I. (2003). Graphite oxide: chemical reduction to graphite and surface modification with primary aliphatic amines and amino acids. Langmuir, 19(15), 6050-6055.

Boussu, K., Belpaire, A., Volodin, A., Van Haesendonck, C., Van Der Meeren, P., Vandecasteele, C., \& Van der Bruggen, B. (2007). Influence of membrane and colloid characteristics on fouling of nanofiltration membranes. Journal of Membrane Science, 289(1), 220-230.

Boussu, K., Vandecasteele, C., \& Van der Bruggen, B. (2008). Relation between membrane characteristics and performance in nanofiltration. Journal of Membrane Science, 310(1), 51-65.

Bowen, W. R., Hilal, N., Lovitt, R. W., \& Williams, P. M. (1996). Atomic Force Microscope Studies of Membranes: Surface Pore Structures of Diaflo Ultrafiltration Membranes. Journal of colloid and interface science, 180(2), 350-359.

Cadotte, J. E., Petersen, R. J., Larson, R. E., \& Erickson, E. E. (1980). A new thin-film composite seawater reverse osmosis membrane. Desalination, 32, 25-31.

Cano, M., Khan, U., Sainsbury, T., O’Neill, A., Wang, Z., McGovern, I. T., . . . Coleman, J. N. (2013). Improving the mechanical properties of graphene oxide based materials by covalent attachment of polymer chains. Carbon, 52, 363371.

Carraro, G., Gasparotto, A., Maccato, C., Bontempi, E., \& Barreca, D. (2015). PECVD of Hematite Nanoblades and Nanocolumns: Synthesis, Characterization, and Growth Model. Chemical Vapor Deposition, 21(10-11-12), 294-299.

Celik, E., Liu, L., \& Choi, H. (2011a). Protein fouling behavior of carbon nanotube/polyethersulfone composite membranes during water filtration. Water Research, 45(16), 5287-5294.

Celik, E., Park, H., Choi, H., \& Choi, H. (2011b). Carbon nanotube blended polyethersulfone membranes for fouling control in water treatment. Water Research, 45(1), 274-282.

Chang, X., Wang, Z., Quan, S., Xu, Y., Jiang, Z., \& Shao, L. (2014). Exploring the synergetic effects of graphene oxide (GO) and polyvinylpyrrodione (PVP) on poly(vinylylidenefluoride) (PVDF) ultrafiltration membrane performance. Applied Surface Science, 316, 537-548.

Chen, C.-M., Chen, M., Leu, F.-C., Hsu, S.-Y., Wang, S.-C., Shi, S.-C., \& Chen, C.-F. (2004). Purification of multi-walled carbon nanotubes by microwave digestion method. Diamond and Related Materials, 13(4-8), 1182-1186. 
Chen, G. E., Xu, S. J., Xu, Z. L., Zhu, W. W., Wu, Q., \& Sun, W. G. (2016). Preparation and characterization of a novel hydrophilic PVDF/PVA UF membrane modified by carboxylated multiwalled carbon nanotubes. Polymer Engineering \& Science, 56(8), 955-967.

Chen, J., \& Lu, G. (2010). Carbon nanotube-nanoparticle hybrid structures: INTECH Open Access Publisher.

Chen, Y., Zhang, Y., Liu, J., Zhang, H., \& Wang, K. (2012). Preparation and antibacterial property of polyethersulfone ultrafiltration hybrid membrane containing halloysite nanotubes loaded with copper ions. Chemical Engineering Journal, 210, 298-308.

Cho, J., Amy, G., \& Pellegrino, J. (1999). Membrane filtration of natural organic matter: initial comparison of rejection and flux decline characteristics with ultrafiltration and nanofiltration membranes. Water Research, 33(11), 25172526.

Choi, O., \& Hu, Z. (2008). Size dependent and reactive oxygen species related nanosilver toxicity to nitrifying bacteria. Environmental Science \& Technology, 42(12), 4583-4588.

Coleman, J. N., Khan, U., Blau, W. J., \& Gun'ko, Y. K. (2006). Small but strong: A review of the mechanical properties of carbon nanotube-polymer composites. Carbon, 44(9), 1624-1652.

Colvin, V. L. (2003). The potential environmental impact of engineered nanomaterials. Nature biotechnology, 21(10), 1166-1170.

Contreras, A. E. (2011). Filtration of Complex Suspensions Using Nanofiltration and Reverse Osmosis Membranes: Foulant-Foulant and Foulant-Membrane Interaction. (PhD.), Rice University.

Cooper, A. R., \& Van Derveer, D. S. (1979). Characterization of ultrafiltration membranes by polymer transport measurements. Separation Science and Technology, 14(6), 551-556.

Dang, T. H. (2009). Surface modifying macromolecules (SMM) - incorporated ultrafiltration membranes for natural organic matter (NOM) removal: characterization and cleaning (Ph.D), University of Ottawa.

Daraei, P., Madaeni, S. S., Ghaemi, N., Salehi, E., Khadivi, M. A., Moradian, R., \& Astinchap, B. (2012). Novel polyethersulfone nanocomposite membrane prepared by $\mathrm{PANI} / \mathrm{Fe} 3 \mathrm{O} 4$ nanoparticles with enhanced performance for $\mathrm{Cu}(\mathrm{II})$ removal from water. Journal of Membrane Science, 415-416, 250-259.

Day, T. M., Unwin, P. R., Wilson, N. R., \& Macpherson, J. V. (2005). Electrochemical templating of metal nanoparticles and nanowires on single-walled carbon nanotube networks. Journal of the American Chemical Society, 127(30), 1063910647.

De Kwaadsteniet, M., Botes, M., \& Cloete, T. E. (2011). Application of Nanotechnology in Antimicrobial Coatings in the Water Industry. Nano, 06, 395-407.

De Matteis, V., Malvindi, M. A., Galeone, A., Brunetti, V., De Luca, E., Kote, S., . . . Pompa, P. P. (2015). Negligible particle-specific toxicity mechanism of silver 
nanoparticles: the role of $\mathrm{Ag}+$ ion release in the cytosol. Nanomedicine, 11(3), 731-739.

Desai, M. A. (2000). Downstream processing of proteins: methods and protocols (Vol. 9): Springer Science \& Business Media.

Devitt, E. Organic Fouling of Membranes; Investigation of the nature of adhesion of organic foulants to membrane surfaces and strategies for cleaning. Retrieved from $\quad$ http://wiesner.cee.duke.edu/research/membrane/organic-foulingmembranes

Dias, A. M., Hussain, A., Marcos, A. S., \& Roque, A. C. (2011). A biotechnological perspective on the application of iron oxide magnetic colloids modified with polysaccharides. Biotechnology Advance, 29(1), 142-155.

Doak, S. H., Liu, Y., \& Chen, C. (2017). Chapter 18 - Genotoxicity and Cancer A2 Fadeel, Bengt. In A. Pietroiusti \& A. A. Shvedova (Eds.), Adverse Effects of Engineered Nanomaterials (Second Edition) (pp. 423-445): Academic Press.

Dong, C., He, G., Li, H., Zhao, R., Han, Y., \& Deng, Y. (2012). Antifouling enhancement of poly(vinylidene fluoride) microfiltration membrane by adding $\mathrm{Mg}(\mathrm{OH}) 2$ nanoparticles. Journal of Membrane Science, 387-388, 40-47.

Donnelly, D., Bergin, J., Duane, T., \& McNulty, N. (1998). Application of Membrane Bioseparation Processes in the Beverage and Food Industries (Vol. I). Germany: Wiley-VCH

Du, R., \& Zhao, J. (2004). Properties of poly (N, N-dimethylaminoethyl methacrylate)/polysulfone positively charged composite nanofiltration membrane. Journal of Membrane Science, 239(2), 183-188.

Einstein, A. (1956). Investigations on the Theory of the Brownian Movement: Courier Corporation.

Elimelech, M., Chen, W. H., \& Waypa, J. J. (1994). Measuring the zeta (electrokinetic) potential of reverse osmosis membranes by a streaming potential analyzer. Desalination, 95(3), 269-286.

Fabrega, J., Fawcett, S. R., Renshaw, J. C., \& Lead, J. R. (2009). Silver nanoparticle impact on bacterial growth: effect of $\mathrm{pH}$, concentration, and organic matter. Environmental Science \& Technology, 43(19), 7285-7290.

Falaras, P., Hugot-Le Goff, A., Bernard, M., \& Xagas, A. (2000). Characterization by resonance Raman spectroscopy of sol-gel TiO 2 films sensitized by the $\mathrm{Ru}(\mathrm{PPh}$ 3) 2 (dcbipy) $\mathrm{Cl} 2$ complex for solar cells application. Solar energy materials and solar cells, 64(2), 167-184.

Faria, A. F., Liu, C., Xie, M., Perreault, F., Nghiem, L. D., Ma, J., \& Elimelech, M. (2017). Thin-film composite forward osmosis membranes functionalized with graphene oxide-silver nanocomposites for biofouling control. Journal of Membrane Science, 525, 146-156.

Fathizadeh, M., Aroujalian, A., \& Raisi, A. (2011). Effect of added NaX nano-zeolite into polyamide as a top thin layer of membrane on water flux and salt rejection in a reverse osmosis process. Journal of Membrane Science, 375(1-2), 88-95. 
Feng, C., Wang, R., Shi, B., Li, G., \& Wu, Y. (2006). Factors affecting pore structure and performance of poly (vinylidene fluoride-co-hexafluoro propylene) asymmetric porous membrane. Journal of Membrane Science, 277(1), 55-64.

Flemming, H.-C. (1997). Reverse osmosis membrane biofouling. Experimental Thermal and Fluid Science, 14(4), 382-391.

Foldbjerg, R., Olesen, P., Hougaard, M., Dang, D. A., Hoffmann, H. J., \& Autrup, H. (2009). PVP-coated silver nanoparticles and silver ions induce reactive oxygen species, apoptosis and necrosis in THP-1 monocytes. Toxicology Letters, 190(2), 156-162.

Fouladgar, M., Beheshti, M., \& Sabzyan, H. (2015). Single and binary adsorption of nickel and copper from aqueous solutions by $\gamma$-alumina nanoparticles: Equilibrium and kinetic modeling. Journal of Molecular Liquids, 211, 10601073.

Fujishima, A., Rao, T. N., \& Tryk, D. A. (2000). Titanium dioxide photocatalysis. Journal of Photochemistry and Photobiology C: Photochemistry Reviews, 1(1), $1-21$.

Ganesh, B. M., Isloor, A. M., \& Ismail, A. F. (2013). Enhanced hydrophilicity and salt rejection study of graphene oxide-polysulfone mixed matrix membrane. Desalination, 313, 199-207.

Ganster, J., \& Fink, H.-P. (2006). Novel cellulose fibre reinforced thermoplastic materials. Cellulose, 13(3), 271-280.

García-Martín, N., Silva, V., Carmona, F., Palacio, L., Hernández, A., \& Prádanos, P. (2014). Pore size analysis from retention of neutral solutes through nanofiltration membranes. The contribution of concentration-polarization. Desalination, 344, 1-11.

Geesey, G. G., Gillis, R. J., Avci, R., Daly, D., Hamilton, M., Shope, P., \& Harkin, G. (1996). The influence of surface features on bacterial colonization and subsequent substratum chemical changes of 316L stainless steel. Corrosion Science, 38(1), 73-95.

Geng, Y., Wang, S. J., \& Kim, J. K. (2009). Preparation of graphite nanoplatelets and graphene sheets. Journal of colloid and interface science, 336(2), 592-598.

Gerard, R., Hachisuka, H., \& Hirose, M. (1998). New membrane developments expanding the horizon for the application of reverse osmosis technology. Desalination, 119(1), 47-55.

Ghaemi, N. (2016). A new approach to copper ion removal from water by polymeric nanocomposite membrane embedded with $\gamma$-alumina nanoparticles. Applied Surface Science, 364, 221-228.

Ghaemi, N., Madaeni, S. S., Daraei, P., Rajabi, H., Zinadini, S., Alizadeh, A., . . . Ghouzivand, S. (2015). Polyethersulfone membrane enhanced with iron oxide nanoparticles for copper removal from water: Application of new functionalized Fe3O4 nanoparticles. Chemical Engineering Journal, 263, 101-112.

Gholami, M., Nasseri, S., Feng, C., Matsuura, T., \& Khulbe, K. (2003). The effect of heat-treatment on the ultrafiltration performance of polyethersulfone (PES) hollow-fiber membranes. Desalination, 155(3), 293-301. 
Gilbert, B., Ono, R. K., Ching, K. A., \& Kim, C. S. (2009). The effects of nanoparticle aggregation processes on aggregate structure and metal uptake. Journal of colloid and interface science, 339(2), 285-295.

Gilron, J., Belfer, S., Väisänen, P., \& Nyström, M. (2001). Effects of surface modification on antifouling and performance properties of reverse osmosis membranes. Desalination, 140(2), 167-179.

Goenka, S., Sant, V., \& Sant, S. (2014). Graphene-based nanomaterials for drug delivery and tissue engineering. Journal of Controlled Release, 173, 75-88.

Goh, P., Lau, W., Othman, M., \& Ismail, A. (2018). Membrane fouling in desalination and its mitigation strategies. Desalination, 425, 130-155.

Gomez, V., Balu, A. M., Serrano-Ruiz, J. C., Irusta, S., Dionysiou, D. D., Luque, R., \& Santamaría, J. (2012). Microwave-assisted mild-temperature preparation of neodymium-doped titania for the improved photodegradation of water contaminants. Applied Catalysis A: General, 441-442, 47-53.

Gong, H., Kim, S.-T., Lee, J. D., \& Yim, S. (2013). Simple quantification of surface carboxylic acids on chemically oxidized multi-walled carbon nanotubes. Applied Surface Science, 266(Supplement C), 219-224.

Gottschalk, F., \& Nowack, B. (2011). The release of engineered nanomaterials to the environment. Journal of Environmental Monitoring, 13(5), 1145-1155.

Gunawan, P., Guan, C., Song, X., Zhang, Q., Leong, S. S. J., Tang, C., . . Wang, K. (2011). Hollow fiber membrane decorated with Ag/MWNTs: toward effective water disinfection and biofouling control. ACS nano, 5(12), 10033-10040.

Gund, G. S., Dubal, D. P., Chodankar, N. R., Cho, J. Y., Gomez-Romero, P., Park, C., \& Lokhande, C. D. (2015). Low-cost flexible supercapacitors with high-energy density based on nanostructured $\mathrm{MnO} 2$ and $\mathrm{Fe} 2 \mathrm{O} 3$ thin films directly fabricated onto stainless steel. Scientific reports, 5.

Gupta, A. K., \& Gupta, M. (2005). Synthesis and surface engineering of iron oxide nanoparticles for biomedical applications. Biomaterials, 26(18), 3995-4021.

Gupta, V., \& Saleh, T. A. (2011). Syntheses of carbon nanotube-metal oxides composites; adsorption and photo-degradation Carbon Nanotubes-From Research to Applications: InTech.

Guzmán, M. G., Dille, J., \& Godet, S. (2009). Synthesis of silver nanoparticles by chemical reduction method and their antibacterial activity. Int J Chem Biomol Eng, 2(3), 104-111.

Han, M.-J., \& Nam, S.-T. (2002). Thermodynamic and rheological variation in polysulfone solution by PVP and its effect in the preparation of phase inversion membrane. Journal of Membrane Science, 202(1), 55-61.

Hanafi, Y., Loulergue, P., Ababou-Girard, S., Meriadec, C., Rabiller-Baudry, M., Baddari, K., \& Szymczyk, A. (2016). Electrokinetic analysis of PES/PVP membranes aged by sodium hypochlorite solutions at different $\mathrm{pH}$. Journal of Membrane Science, 501, 24-32.

Hanemann, T., \& Szabó, D. V. (2010). Polymer-nanoparticle composites: from synthesis to modern applications. Materials, 3(6), 3468-3517. 
Helin, H., Na, L., Linlin, W., ZHONG, H., Guangxia, W., Zonghuan, Y., . . Lianyi, T. (2008). Anti-fouling ultrafiltration membrane prepared from polysulfone-graftmethyl acrylate copolymers by UV-induced grafting method. Journal of Environmental Sciences, 20(5), 565-570.

Hemalatha, K., Prakash, A., Guruprakash, K., \& Jayakumar, M. (2014). TiO 2 coated carbon nanotubes for electrochemical energy storage. Journal of Materials Chemistry A, 2(6), 1757-1766.

Henglein, A. (2001). Reduction of Ag (CN) 2-on silver and platinum colloidal nanoparticles. Langmuir, 17(8), 2329-2333.

Henriksson, M., \& Berglund, L. A. (2007). Structure and properties of cellulose nanocomposite films containing melamine formaldehyde. Journal of applied polymer science, 106(4), 2817-2824.

Hilal, N., Ismail, A. F., Matsuura, T., \& Oatley-Radcliffe, D. L. (2017). Membrane Characterization: Elsevier.

Hilal, N., Ismail, A. F., \& Wright, C. (2015). Membrane Fabrication: CRC Press.

Hilal, N., Khayet, M., \& Wright, C. J. (2012). Membrane modification: Technology and applications: CRC Press.

Hilal, N., Ogunbiyi, O. O., Miles, N. J., \& Nigmatullin, R. (2005). Methods Employed for Control of Fouling in MF and UF Membranes: A Comprehensive Review. Separation Science and Technology, 40(10), 1957-2005.

Homayoonfal, M., Mehrnia, M. R., Rahmani, S., \& Mohades Mojtahedi, Y. (2015). Fabrication of alumina/polysulfone nanocomposite membranes with biofouling mitigation approach in membrane bioreactors. Journal of Industrial and Engineering Chemistry, 22, 357-367.

Homayoonfal, M., Mehrnia, M. R., Shariaty-Niassar, M., Akbari, A., Ismail, A. F., \& Matsuura, T. (2014). A comparison between blending and surface deposition methods for the preparation of iron oxide/polysulfone nanocomposite membranes. Desalination, 354, 125-142.

Hong, S., \& Elimelech, M. (1997). Chemical and physical aspects of natural organic matter (NOM) fouling of nanofiltration membranes. Journal of Membrane Science, 132(2), 159-181.

Hosokawa, M., Nogi, K., Naito, M., \& Yokayama, T. (2007). Nanoparticle technology handbook: Elsevier BV.

Hu, W., Peng, C., Luo, W., Lv, M., Li, X., Li, D., . . Fan, C. (2010). Graphene-based antibacterial paper. ACS nano, 4(7), 4317-4323.

Hu, Y., \& Guo, C. (2011). Carbon nanotubes and carbon nanotubes/metal oxide heterostructures: synthesis, characterization and electrochemical property Carbon Nanotubes-Growth and Applications: InTech.

Hua, H., Li, N., Wu, L., Zhong, H., Wu, G., Yuan, Z., . . Tang, L. (2008). Anti-fouling ultrafiltration membrane prepared from polysulfone-graft-methyl acrylate copolymers by UV-induced grafting method. Journal of Environmental Sciences, 20(5), 565-570. 
Huang, Z., Cui, F., Kang, H., Chen, J., Zhang, X., \& Xia, C. (2008). Highly dispersed silica-supported copper nanoparticles prepared by precipitation- gel method: a simple but efficient and stable catalyst for glycerol hydrogenolysis. Chemistry of Materials, 20(15), 5090-5099.

Huisman, I. H., Prádanos, P., \& Hernández, A. (2000). The effect of protein-protein and protein-membrane interactions on membrane fouling in ultrafiltration. Journal of Membrane Science, 179(1), 79-90.

Ikeda, K., Nakano, T., Ito, H., Kubota, T., \& Yamamoto, S. (1988). New composite charged reverse osmosis membrane. Desalination, 68(2), 109-119.

Imtani, A. N., \& Jindal, V. (2006). Bond Lengths of Single-Walled Carbon Nanotubes. arXiv preprint cond-mat/0611484.

Ingole, P. G., Choi, W., Kim, K. H., Park, C. H., Choi, W. K., \& Lee, H. K. (2014). Synthesis, characterization and surface modification of PES hollow fiber membrane support with polydopamine and thin film composite for energy generation. Chemical Engineering Journal, 243, 137-146.

Iravani, S., Korbekandi, H., Mirmohammadi, S., \& Zolfaghari, B. (2014). Synthesis of silver nanoparticles: chemical, physical and biological methods. Research in pharmaceutical sciences, 9(6), 385.

Isloor, A. M., Ganesh, B. M., Isloor, S. M., Ismail, A. F., Nagaraj, H. S., \& Pattabi, M. (2013). Studies on copper coated polysulfone/modified poly isobutylene altmaleic anhydride blend membrane and its antibiofouling property. Desalination, 308, 82-88.

Ismail, A. F., Padaki, M., Hilal, N., Matsuura, T., \& Lau, W. J. (2015). Thin film composite membrane - Recent development and future potential. Desalination, 356(Supplement C), 140-148.

Israelachvili, J. N. (1992). Intermolecular and Surface Forces: With Applications to Colloidal and Biological Systems (Colloid Science). (2nd ed.). London: Academic press.

Jeong, B.-H., Hoek, E. M., Yan, Y., Subramani, A., Huang, X., Hurwitz, G., . . Jawor, A. (2007). Interfacial polymerization of thin film nanocomposites: a new concept for reverse osmosis membranes. Journal of Membrane Science, 294(1), $1-7$.

Jesumathy, S., Udayakumar, M., \& Suresh, S. (2011). Experimental study of enhanced heat transfer by addition of $\mathrm{CuO}$ nanoparticle. Heat and Mass Transfer, 48(6), 965-978.

Jhaveri, J. H., \& Murthy, Z. V. P. (2016). A comprehensive review on anti-fouling nanocomposite membranes for pressure driven membrane separation processes. Desalination, 379, 137-154.

Ji, B.-h. (2012). Structural Characterization for Carbon Nanotubes Treated with Concentrated Nitric Acid. Asian Journal of Chemistry, 24(10), 4271.

Johnston, H. J., Hutchison, G., Christensen, F. M., Peters, S., Hankin, S., \& Stone, V. (2010). A review of the in vivo and in vitro toxicity of silver and gold particulates: particle attributes and biological mechanisms responsible for the observed toxicity. Critical Reviews in Toxicology, 40(4), 328-346. 
Jönsson, C., \& Jönsson, A.-S. (1995). Influence of the membrane material on the adsorptive fouling of ultrafiltration membranes. Journal of Membrane Science, 108(1), 79-87.

Kamal, A., Srinivasulu, V., Murty, J. N. S. R. C., Shankaraiah, N., Nagesh, N., Srinivasa Reddy, T., \& Subba Rao, A. V. (2013). Copper Oxide Nanoparticles Supported on Graphene Oxide- Catalyzed S-Arylation: An Efficient and Ligand-Free Synthesis of Aryl Sulfides. Advanced Synthesis \& Catalysis, 355(11-12), 2297-2307.

Kang, E., Lee, Y., Chon, K., \& Cho, J. (2014). Effects of hydrodynamic conditions (diffusion vs. convection) and solution chemistry on effective molecular weight cut-off of negatively charged nanofiltration membranes. Desalination, 352(Supplement C), 136-141.

Kang, S., Herzberg, M., Rodrigues, D. F., \& Elimelech, M. (2008a). Antibacterial effects of carbon nanotubes: size does matter! Langmuir, 24(13), 6409-6413.

Kang, X., Mai, Z., Zou, X., Cai, P., \& Mo, J. (2008b). Glucose biosensors based on platinum nanoparticles-deposited carbon nanotubes in sol-gel chitosan/silica hybrid. Talanta, 74(4), 879-886.

Kango, S., Kalia, S., Celli, A., Njuguna, J., Habibi, Y., \& Kumar, R. (2013). Surface modification of inorganic nanoparticles for development of organic-inorganic nanocomposites-A review. Progress in Polymer Science, 38(8), 1232-1261.

Kaszuba, M., Corbett, J., Watson, F. M., \& Jones, A. (2010). High-concentration zeta potential measurements using light-scattering techniques. Philos Trans A Math Phys Eng Sci, 368(1927), 4439-4451. doi:10.1098/rsta.2010.0175

Khalid, A., Al-Juhani, A. A., Al-Hamouz, O. C., Laoui, T., Khan, Z., \& Atieh, M. A. (2015). Preparation and properties of nanocomposite polysulfone/multi-walled carbon nanotubes membranes for desalination. Desalination, 367, 134-144.

Khayet, M., \& Matsuura, T. (2003). Determination of surface and bulk pore sizes of flat-sheet and hollow-fiber membranes by atomic force microscopy, gas permeation and solute transport methods. Desalination, 158(1), 57-64.

Khulbe, K., Feng, C., \& Matsuura, T. (2010). The art of surface modification of synthetic polymeric membranes. Journal of applied polymer science, 115(2), 855-895.

Kim, B., \& Sigmund, W. M. (2004). Functionalized multiwall carbon nanotube/gold nanoparticle composites. Langmuir, 20(19), 8239-8242.

Kim, E.-S., \& Deng, B. (2011). Fabrication of polyamide thin-film nano-composite (PA-TFN) membrane with hydrophilized ordered mesoporous carbon (HOMC) for water purifications. Journal of Membrane Science, 375(1), 46-54.

Kim, E.-S., Hwang, G., Gamal El-Din, M., \& Liu, Y. (2012). Development of nanosilver and multi-walled carbon nanotubes thin-film nanocomposite membrane for enhanced water treatment. Journal of Membrane Science, 394$395,37-48$.

Kim, J., Kang, M., \& Kim, C. (2005). Fabrication of membranes for the liquid separation: Part 1. Ultrafiltration membranes prepared from novel miscible 
blends of polysulfone and poly (1-vinylpyrrolidone-co-acrylonitrile) copolymers. Journal of Membrane Science, 265(1), 167-175.

Kim, J., \& Van der Bruggen, B. (2010). The use of nanoparticles in polymeric and ceramic membrane structures: review of manufacturing procedures and performance improvement for water treatment. Environmental Pollution, $158(7), 2335-2349$.

Kim, J. S., Kuk, E., Yu, K. N., Kim, J. H., Park, S. J., Lee, H. J., . . Cho, M. H. (2007). Antimicrobial effects of silver nanoparticles. Nanomedicine, 3(1), 95-101.

Kim, J. Y., Lee, C., Cho, M., \& Yoon, J. (2008). Enhanced inactivation of E. coli and MS-2 phage by silver ions combined with UV-A and visible light irradiation. Water Research, 42(1-2), 356-362.

Kim, S., Choi, J. E., Choi, J., Chung, K.-H., Park, K., Yi, J., \& Ryu, D.-Y. (2009). Oxidative stress-dependent toxicity of silver nanoparticles in human hepatoma cells. Toxicology in Vitro, 23(6), 1076-1084.

Kim, S. H., Kwak, S.-Y., Sohn, B.-H., \& Park, T. H. (2003). Design of TiO 2 nanoparticle self-assembled aromatic polyamide thin-film-composite (TFC) membrane as an approach to solve biofouling problem. Journal of Membrane Science, 211(1), 157-165.

Kimura, K., Hane, Y., Watanabe, Y., Amy, G., \& Ohkuma, N. (2004). Irreversible membrane fouling during ultrafiltration of surface water. Water Research, 38(14-15), 3431-3441.

Klemm, D. (2006). Polysaccharides II (Vol. 205): Springer.

Kochkodan, V., \& Hilal, N. (2015). A comprehensive review on surface modified polymer membranes for biofouling mitigation. Desalination, 356, 187-207.

Kochkodan, V., Hilal, N., Goncharuk, V., Al-Khatib, L., \& Levadna, T. (2006). Effect of the surface modification of polymer membranes on their microbiological fouling. Colloid Journal, 68(3), 267-273.

Kochkodan, V., Johnson, D. J., \& Hilal, N. (2014). Polymeric membranes: surface modification for minimizing (bio)colloidal fouling. Advances in Colloid and Interface Science, 206, 116-140.

Koehler, J. A., Ulbricht, M., \& Belfort, G. (2000). Intermolecular forces between a protein and a hydrophilic modified polysulfone film with relevance to filtration. Langmuir, 16(26), 10419-10427.

Koli, V. B., Dhodamani, A. G., Delekar, S. D., \& Pawar, S. H. (2017). In situ sol-gel synthesis of anatase TiO 2-MWCNTs nanocomposites and their photocatalytic applications. Journal of photochemistry and photobiology A: Chemistry, 333, 40-48.

Kuilla, T., Bhadra, S., Yao, D., Kim, N. H., Bose, S., \& Lee, J. H. (2010). Recent advances in graphene based polymer composites. Progress in Polymer Science, 35(11), 1350-1375.

Kumar, A. P., Depan, D., Singh Tomer, N., \& Singh, R. P. (2009). Nanoscale particles for polymer degradation and stabilization-Trends and future perspectives. Progress in Polymer Science, 34(6), 479-515. 
Kundu, S., Wang, Y., Xia, W., \& Muhler, M. (2008). Thermal Stability and Reducibility of Oxygen-Containing Functional Groups on Multiwalled Carbon Nanotube Surfaces: A Quantitative High-Resolution XPS and TPD/TPR Study. The Journal of Physical Chemistry C, 112(43), 16869-16878. doi:10.1021/jp804413a

Lalia, B. S., Kochkodan, V., Hashaikeh, R., \& Hilal, N. (2013). A review on membrane fabrication: Structure, properties and performance relationship. Desalination, $326,77-95$.

Lau, W., Gray, S., Matsuura, T., Emadzadeh, D., Chen, J. P., \& Ismail, A. (2015). A review on polyamide thin film nanocomposite (TFN) membranes: history, applications, challenges and approaches. Water Research, 80, 306-324.

Le-Clech, P., Chen, V., \& Fane, T. A. (2006). Fouling in membrane bioreactors used in wastewater treatment. Journal of Membrane Science, 284(1), 17-53.

Lee, H. S., Im, S. J., Kim, J. H., Kim, H. J., Kim, J. P., \& Min, B. R. (2008). Polyamide thin-film nanofiltration membranes containing $\mathrm{TiO} 2$ nanoparticles. Desalination, 219(1-3), 48-56.

Lee, J., Chae, H.-R., Won, Y. J., Lee, K., Lee, C.-H., Lee, H. H., . . Lee, J.-m. (2013). Graphene oxide nanoplatelets composite membrane with hydrophilic and antifouling properties for wastewater treatment. Journal of Membrane Science, 448, 223-230.

Li, J.-H., Xu, Y.-Y., Zhu, L.-P., Wang, J.-H., \& Du, C.-H. (2009). Fabrication and characterization of a novel $\mathrm{TiO} 2$ nanoparticle self-assembly membrane with improved fouling resistance. Journal of Membrane Science, 326(2), 659-666.

Li, J., Tang, S., Lu, L., \& Zeng, H. C. (2007a). Preparation of nanocomposites of metals, metal oxides, and carbon nanotubes via self-assembly. Journal of the American Chemical Society, 129(30), 9401-9409.

Li, L., Yang, Y., Yang, G., Chen, X., Hsiao, B. S., Chu, B., . . . Li, C. Y. (2006). Patterning polyethylene oligomers on carbon nanotubes using physical vapor deposition. Nano letters, 6(5), 1007-1012.

Li, M., \& He, H. (2017). Study on electrochemical performance of multi-wall carbon nanotubes coated by iron oxide nanoparticles as advanced electrode materials for supercapacitors. Vacuum, 143, 371-379.

Li, Q., Mahendra, S., Lyon, D. Y., Brunet, L., Liga, M. V., Li, D., \& Alvarez, P. J. (2008). Antimicrobial nanomaterials for water disinfection and microbial control: potential applications and implications. Water Research, 42(18), 45914602 .

Li, Q., Xu, Z., \& Pinnau, I. (2007b). Fouling of reverse osmosis membranes by biopolymers in wastewater secondary effluent: Role of membrane surface properties and initial permeate flux. Journal of Membrane Science, 290(1), 173181.

Li, S., Gao, Y., Bai, H., Zhang, L., Qu, P., \& Bai, L. (2011). Preparation and characteristics of polysulfone dialysis composite membranes modified with nanocrystalline cellulose. BioResources, 6(2), 1670-1680. 
Li, Y., Doak, S. H., Yan, J., Chen, D. H., Zhou, M., Mittelstaedt, R. A., . . Chen, T. (2017). Factors affecting the in vitro micronucleus assay for evaluation of nanomaterials. Mutagenesis, 32(1), 151-159. doi:10.1093/mutage/gew040

Liebscher, J. r., Mrówczyński, R., Scheidt, H. A., Filip, C., Hădade, N. D., Turcu, R., . .. Beck, S. (2013). Structure of polydopamine: a never-ending story? Langmuir, 29(33), 10539-10548.

Lim, Y. P., \& Mohammad, A. W. (2010). Effect of solution chemistry on flux decline during high concentration protein ultrafiltration through a hydrophilic membrane. Chemical Engineering Journal, 159(1), 91-97.

Lin, Y., Baggett, D. W., Kim, J.-W., Siochi, E. J., \& Connell, J. W. (2011). Instantaneous formation of metal and metal oxide nanoparticles on carbon nanotubes and graphene via solvent-free microwave heating. ACS applied materials \& interfaces, 3(5), 1652-1664.

Lin, Y., Watson, K. A., Fallbach, M. J., Ghose, S., Smith Jr, J. G., Delozier, D. M., . . Connell, J. W. (2009). Rapid, solventless, bulk preparation of metal nanoparticle-decorated carbon nanotubes. ACS nano, 3(4), 871-884.

Liu, C., Faria, A. F., Ma, J., \& Elimelech, M. (2017). Mitigation of Biofilm Development on Thin-Film Composite Membranes Functionalized with Zwitterionic Polymers and Silver Nanoparticles. Environ Sci Technol, 51(1), 182-191.

Liu, C., Shi, L., \& Wang, R. (2015). Enhanced hollow fiber membrane performance via semi-dynamic layer-by-layer polyelectrolyte inner surface deposition for nanofiltration and forward osmosis applications. Reactive and Functional Polymers, 86, 154-160.

Liu, C. X., Zhang, D. R., He, Y., Zhao, X. S., \& Bai, R. (2010). Modification of membrane surface for anti-biofouling performance: Effect of anti-adhesion and anti-bacteria approaches. Journal of Membrane Science, 346(1), 121-130.

Liu, S., Yao, K., Fu, L.-H., \& Ma, M.-G. (2016). Selective synthesis of Fe 3 O 4, $\gamma$-Fe $2 \mathrm{O} 3$, and $\alpha-\mathrm{Fe} 2 \mathrm{O} 3$ using cellulose-based composites as precursors. RSC Advances, 6(3), 2135-2140.

Loeb, S., \& Sourirajan, S. (1963). Sea Water Demineralization by Means of an Osmotic Membrane Saline Water Conversion-II (Vol. 38, pp. 117-132): AMERICAN CHEMICAL SOCIETY.

Lordi, V., Yao, N., \& Wei, J. (2001). Method for supporting platinum on single-walled carbon nanotubes for a selective hydrogenation catalyst. Chemistry of Materials, 13(3), 733-737.

Lv, Y., Du, Y., Qiu, W.-Z., \& Xu, Z.-K. (2017). Nanocomposite Membranes via the Codeposition of Polydopamine/Polyethylenimine with Silica Nanoparticles for Enhanced Mechanical Strength and High Water Permeability. ACS applied materials \& interfaces, 9(3), 2966-2972.

Lv, Y., Yang, H.-C., Liang, H.-Q., Wan, L.-S., \& Xu, Z.-K. (2016). Novel nanofiltration membrane with ultrathin zirconia film as selective layer. Journal of Membrane Science, 500, 265-271. 
Madaeni, S. S., \& Ghaemi, N. (2007). Characterization of self-cleaning RO membranes coated with $\mathrm{TiO} 2$ particles under UV irradiation. Journal of Membrane Science, 303(1-2), 221-233.

Madigan, M., \& Martinko, J. (2005). Brock Biology of Microorganisms. 11th EditionPrentice Hall: USA.

Mänttäri, M., Puro, L., Nuortila-Jokinen, J., \& Nyström, M. (2000). Fouling effects of polysaccharides and humic acid in nanofiltration. Journal of Membrane Science, 165(1), 1-17.

María Arsuaga, J., Sotto, A., del Rosario, G., Martínez, A., Molina, S., Teli, S. B., \& de Abajo, J. (2013). Influence of the type, size, and distribution of metal oxide particles on the properties of nanocomposite ultrafiltration membranes. Journal of Membrane Science, 428, 131-141.

Mauter, M. S., Wang, Y., Okemgbo, K. C., Osuji, C. O., Giannelis, E. P., \& Elimelech, M. (2011). Antifouling ultrafiltration membranes via post-fabrication grafting of biocidal nanomaterials. ACS applied materials \& interfaces, 3(8), 28612868 .

Maximous, N., Nakhla, G., Wan, W., \& Wong, K. (2009). Preparation, characterization and performance of $\mathrm{Al} 2 \mathrm{O} 3 / \mathrm{PES}$ membrane for wastewater filtration. Journal of Membrane Science, 341(1), 67-75.

Maximous, N., Nakhla, G., Wan, W., \& Wong, K. (2010). Performance of a novel $\mathrm{ZrO} 2 / \mathrm{PES}$ membrane for wastewater filtration. Journal of Membrane Science, 352(1-2), 222-230.

Medda, S., Hajra, A., Dey, U., Bose, P., \& Mondal, N. K. (2014). Biosynthesis of silver nanoparticles from Aloe vera leaf extract and antifungal activity against Rhizopus sp. and Aspergillus sp. Applied Nanoscience, 5(7), 875-880.

Mehrnia, M. R., Mojtahedi, Y. M., \& Homayoonfal, M. (2015). What is the concentration threshold of nanoparticles within the membrane structure? A case study of Al2O3/PSf nanocomposite membrane. Desalination, 372, 75-88.

Michaels, A. S. (1980). Analysis and prediction of sieving curves for ultrafiltration membranes: A universal correlation? Separation Science and Technology, 15(6), 1305-1322.

Mierzwa, J. C., Arieta, V., Verlage, M., Carvalho, J., \& Vecitis, C. D. (2013). Effect of clay nanoparticles on the structure and performance of polyethersulfone ultrafiltration membranes. Desalination, 314, 147-158.

Mills, A., \& Le Hunte, S. (1997). An overview of semiconductor photocatalysis. Journal of photochemistry and photobiology A: Chemistry, 108(1), 1-35.

Mittal, K. L. (2007). Silanes and other coupling agents (Vol. 4): CRC Press.

Mkhondo, N., \& Magadzu, T. (2014). Effects of different acid-treatment on the nanostructure and performance of carbon nanotubes in electrochemical hydrogen storage. Digest Journal of Nanomaterials \& Biostructures (DJNB), 9(4).

Mo, H., Tay, K. G., \& Ng, H. Y. (2008). Fouling of reverse osmosis membrane by protein (BSA): effects of $\mathrm{pH}$, calcium, magnesium, ionic strength and temperature. Journal of Membrane Science, 315(1), 28-35. 
Mohammad, A. W., Hilal, N., \& Seman, M. N. A. (2003). A study on producing composite nanofiltration membranes with optimized properties. Desalination, 158(1-3), 73-78.

Mohammad, A. W., Othaman, R., \& Hilal, N. (2004). Potential use of nanofiltration membranes in treatment of industrial wastewater from Ni-P electroless plating. Desalination, 168, 241-252.

Mohammad, A. W., Teow, Y. H., Ang, W. L., Chung, Y. T., Oatley-Radcliffe, D. L., \& Hilal, N. (2015). Nanofiltration membranes review: Recent advances and future prospects. Desalination, 356, 226-254.

Mollahosseini, A., Rahimpour, A., Jahamshahi, M., Peyravi, M., \& Khavarpour, M. (2012). The effect of silver nanoparticle size on performance and antibacteriality of polysulfone ultrafiltration membrane. Desalination, 306, 4150 .

Moraes, F., Cabral, M., Mascaro, L., \& Machado, S. (2011). The electrochemical effect of acid functionalisation of carbon nanotubes to be used in sensors development. Surface Science, 605(3), 435-440.

Mosqueda-Jimenez, D. B., Narbaitz, R. M., Matsuura, T., Chowdhury, G., Pleizier, G., \& Santerre, J. P. (2004). Influence of processing conditions on the properties of ultrafiltration membranes. Journal of Membrane Science, 231(1-2), 209-224.

Mukherjee, P., Jones, K., \& Abitoye, J. (2005a). Surface modification of nanofiltration membranes by ion implantation. Journal of Membrane Science, 254(1-2), 303310 .

Mukherjee, P., Jones, K. L., \& Abitoye, J. O. (2005b). Surface modification of nanofiltration membranes by ion implantation. Journal of Membrane Science, 254(1), 303-310.

Mulder, M. (1996). Basic Principles of Membrane Technology: Springer.

Nackaerts, R. (2014). Are membranes implemented with nanoparticles able to provide a breakthrough in water purification? (M.Sc), University of Johannesburg.

Nair, A. K., Isloor, A. M., Kumar, R., \& Ismail, A. (2013). Antifouling and performance enhancement of polysulfone ultrafiltration membranes using $\mathrm{CaCO} 3$ nanoparticles. Desalination, 322, 69-75.

Navarro, E., Piccapietra, F., Wagner, B., Marconi, F., Kaegi, R., Odzak, N., . . Behra, R. (2008). Toxicity of silver nanoparticles to Chlamydomonas reinhardtii. Environmental Science \& Technology, 42(23), 8959-8964.

Ng, L. Y., Mohammad, A. W., Leo, C. P., \& Hilal, N. (2013). Polymeric membranes incorporated with metal/metal oxide nanoparticles: A comprehensive review. Desalination, 308, 15-33.

Nyström, M., Ruohomäki, K., \& Kaipia, L. (1996). Humic acid as a fouling agent in filtration. Desalination, 106(1-3), 79-87.

O'Gorman, J., \& Humphreys, H. (2012). Application of copper to prevent and control infection. Where are we now? Journal of Hospital Infection, 81(4), 217-223.

Ochoa, N., Pradanos, P., Palacio, L., Pagliero, C., Marchese, J., \& Hernandez, A. (2001). Pore size distributions based on AFM imaging and retention of 
multidisperse polymer solutes: characterisation of polyethersulfone UF membranes with dopes containing different PVP. Journal of Membrane Science, 187(1), 227-237.

Okada, A., \& Usuki, A. (1995). The chemistry of polymer-clay hybrids. Materials Science and Engineering: C, 3(2), 109-115.

Orishchin, N., Crane, C. C., Brownell, M., Wang, T., Jenkins, S., Zou, M., . . Chen, J. (2017). Rapid Deposition of Uniform Polydopamine Coatings on Nanoparticle Surfaces with Controllable Thickness. Langmuir, 33(24), 6046-6053.

Otero, J. A., Mazarrasa, O., Villasante, J., Silva, V., Prádanos, P., Calvo, J. I., \& Hernández, A. (2008). Three independent ways to obtain information on pore size distributions of nanofiltration membranes. Journal of Membrane Science, 309(1-2), 17-27.

Paine, P. L., \& Scherr, P. (1975). Drag coefficients for the movement of rigid spheres through liquid-filled cylindrical pores. Biophysical journal, 15(10), 1087-1091.

Pal, S., Tak, Y. K., \& Song, J. M. (2007). Does the antibacterial activity of silver nanoparticles depend on the shape of the nanoparticle? A study of the Gramnegative bacterium Escherichia coli. Applied and Enviromental Microbiology, 73(6), 1712-1720.

Panáček, A., Kvitek, L., Prucek, R., Kolar, M., Vecerova, R., Pizurova, N., . . Zboril, R. (2006). Silver colloid nanoparticles: synthesis, characterization, and their antibacterial activity. The Journal of Physical Chemistry B, 110(33), 1624816253.

Park, E. J., Yi, J., Kim, Y., Choi, K., \& Park, K. (2010). Silver nanoparticles induce cytotoxicity by a Trojan-horse type mechanism. Toxicol In Vitro, 24(3), 872878.

Pathania, D., Katwal, R., Sharma, G., Naushad, M., Khan, M. R., \& Al-Muhtaseb, A. H. (2016). Novel guar gum/Al2O3 nanocomposite as an effective photocatalyst for the degradation of malachite green dye. International Journal of Biological Macromolecules, 87, 366-374.

Pendergast, M. M., \& Hoek, E. M. (2011). A review of water treatment membrane nanotechnologies. Energy \& Environmental Science, 4(6), 1946-1971.

Pincet, F., Perez, E., \& Belfort, G. (1994). Do denatured proteins behave like polymers? Macromolecules, 27(12), 3424-3425.

Plueddemann, E. P. (2013). Silane coupling agents: Springer Science \& Business Media.

Powell, L. C., Hilal, N., \& Wright, C. J. (2017). Atomic force microscopy study of the biofouling and mechanical properties of virgin and industrially fouled reverse osmosis membranes. Desalination, 404(Supplement C), 313-321.

Prince, J. A., Bhuvana, S., Boodhoo, K. V. K., Anbharasi, V., \& Singh, G. (2014). Synthesis and characterization of PEG-Ag immobilized PES hollow fiber ultrafiltration membranes with long lasting antifouling properties. Journal of Membrane Science, 454, 538-548. 
Qin, J.-J., Oo, M. H., Lee, H., \& Coniglio, B. (2004). Effect of feed pH on permeate $\mathrm{pH}$ and ion rejection under acidic conditions in NF process. Journal of Membrane Science, 232(1), 153-159.

Qin, J.-J., Oo, M. H., \& Li, Y. (2005). Development of high flux polyethersulfone hollow fiber ultrafiltration membranes from a low critical solution temperature dope via hypochlorite treatment. Journal of Membrane Science, 247(1), 137142.

Qu, X., Alvarez, P. J., \& Li, Q. (2013). Applications of nanotechnology in water and wastewater treatment. Water Research, 47(12), 3931-3946.

Quan, H., Zhang, B.-q., Zhao, Q., Yuen, R. K. K., \& Li, R. K. Y. (2009). Facile preparation and thermal degradation studies of graphite nanoplatelets (GNPs) filled thermoplastic polyurethane (TPU) nanocomposites. Composites Part A: Applied Science and Manufacturing, 40(9), 1506-1513.

Quinn, B. M., Dekker, C., \& Lemay, S. G. (2005). Electrodeposition of noble metal nanoparticles on carbon nanotubes. Journal of the American Chemical Society, 127(17), 6146-6147.

Rahimpour, A., Jahanshahi, M., Khalili, S., Mollahosseini, A., Zirepour, A., \& Rajaeian, B. (2012). Novel functionalized carbon nanotubes for improving the surface properties and performance of polyethersulfone (PES) membrane. Desalination, 286, 99-107.

Rahimpour, A., Jahanshahi, M., Mortazavian, N., Madaeni, S. S., \& Mansourpanah, Y. (2010). Preparation and characterization of asymmetric polyethersulfone and thin-film composite polyamide nanofiltration membranes for water softening. Applied Surface Science, 256(6), 1657-1663.

Rahimpour, A., Madaeni, S. S., Jahanshahi, M., Mansourpanah, Y., \& Mortazavian, N. (2009). Development of high performance nano-porous polyethersulfone ultrafiltration membranes with hydrophilic surface and superior antifouling properties. Applied Surface Science, 255(22), 9166-9173.

Rahimpour, A., Madaeni, S. S., Taheri, A. H., \& Mansourpanah, Y. (2008). Coupling TiO2 nanoparticles with UV irradiation for modification of polyethersulfone ultrafiltration membranes. Journal of Membrane Science, 313(1-2), 158-169.

Rajaeian, B., Rahimpour, A., Tade, M. O., \& Liu, S. (2013). Fabrication and characterization of polyamide thin film nanocomposite (TFN) nanofiltration membrane impregnated with TiO2 nanoparticles. Desalination, 313, 176-188.

Raman, L. P., Cheryna, M., \& Rajagopalan, N. (1994). Consider nanofiltration for membrane separations. Chemical Engineering Progress;(United States), 90(3).

Rambabu, K., \& Velu, S. (2014). Iron nanoparticles blended polyethersulfone ultrafiltration membranes for enhanced metal ion removal in wastewater treatment. International Journal of ChemTech Research, 6(10), 4468-4470.

Ramyadevi, J., Jeyasubramanian, K., Marikani, A., Rajakumar, G., \& Rahuman, A. A. (2012). Synthesis and antimicrobial activity of copper nanoparticles. Materials Letters, 71, 114-116.

Rana, D., \& Matsuura, T. (2010). Surface modifications for antifouling membranes. Chemical reviews, 110(4), 2448-2471. 
Rao, C. N. R., Müller, A., \& Cheetham, A. K. (2006). The chemistry of nanomaterials: synthesis, properties and applications: John Wiley \& Sons.

Razmjou, A., Mansouri, J., \& Chen, V. (2011). The effects of mechanical and chemical modification of TiO 2 nanoparticles on the surface chemistry, structure and fouling performance of PES ultrafiltration membranes. Journal of Membrane Science, 378(1), 73-84.

Reddy, N., Bera, P., Reddy, V. R., Sridhara, N., Dey, A., Anandan, C., \& Sharma, A. K. (2014). XPS study of sputtered alumina thin films. Ceramics International, 40(7), 11099-11107.

Rehan, M., Mashaly, H. M., Mowafi, S., El-Kheir, A. A., \& Emam, H. E. (2015). Multifunctional textile design using in-situ Ag NPs incorporation into natural fabric matrix. Dyes and Pigments, 118, 9-17.

Reiss, C. R., Taylor, J. S., \& Robert, C. (1999). Surface water treatment using nanofiltration - pilot testing results and design considerations. Desalination, 125(1), 97-112.

Renault, O., Gosset, L. G., Rouchon, D., \& Ermolieff, A. (2002). Angle-resolved x-ray photoelectron spectroscopy of ultrathin $\mathrm{A} 12 \mathrm{O} 3$ films grown by atomic layer deposition. Journal of Vacuum Science \& Technology A: Vacuum, Surfaces, and Films, 20(6), 1867-1876.

Rhee, S. K., \& Steinbuchel, A. (2005). Polysaccharides and polyamides in the food industry: properties, production, and patents: Wiley-Vch.

Rohani, R., Hyland, M., \& Patterson, D. (2011). A refined one-filtration method for aqueous based nanofiltration and ultrafiltration membrane molecular weight cut-off determination using polyethylene glycols. Journal of Membrane Science, 382(1), 278-290.

Roy, D., Tiwari, N., Kanojia, S., Mukhopadhyay, K., \& Saxena, A. (2015). Insight into the Mechanism of Decontamination and Disinfection at the Functionalized Carbon Nanotube-Polymer Interfaces. The Journal of Physical Chemistry C, 119(29), 16678-16687.

Ruparelia, J. P., Chatterjee, A. K., Duttagupta, S. P., \& Mukherji, S. (2008). Strain specificity in antimicrobial activity of silver and copper nanoparticles. Acta Biomaterialia, 4(3), 707-716.

Sahoo, N. G., Rana, S., Cho, J. W., Li, L., \& Chan, S. H. (2010). Polymer nanocomposites based on functionalized carbon nanotubes. Progress in Polymer Science, 35(7), 837-867.

Sathish Kumar, R., Arthanareeswaran, G., Paul, D., \& Kweon, J. H. (2015). Modification methods of polyethersulfone membranes for minimizing fouling Review. Membrane Water Treatment, 6(4), 323-337.

Seidel, A., \& Elimelech, M. (2002). Coupling between chemical and physical interactions in natural organic matter (NOM) fouling of nanofiltration membranes: implications for fouling control. Journal of Membrane Science, 203(1), 245-255. 
Shaikjee, A., \& Coville, N. J. (2012). The synthesis, properties and uses of carbon materials with helical morphology. Journal of Advanced Research, 3(3), 195223.

Shao, L., Wang, Z. X., Zhang, Y. L., Jiang, Z. X., \& Liu, Y. Y. (2014). A facile strategy to enhance PVDF ultrafiltration membrane performance via self-polymerized polydopamine followed by hydrolysis of ammonium fluotitanate. Journal of Membrane Science, 461, 10-21.

Shawky, H. A., Chae, S.-R., Lin, S., \& Wiesner, M. R. (2011). Synthesis and characterization of a carbon nanotube/polymer nanocomposite membrane for water treatment. Desalination, 272(1-3), 46-50.

Shen, J. n., Yu, C. c., Ruan, H. m., Gao, C. j., \& Van der Bruggen, B. (2013). Preparation and characterization of thin-film nanocomposite membranes embedded with poly(methyl methacrylate) hydrophobic modified multiwalled carbon nanotubes by interfacial polymerization. Journal of Membrane Science, $442,18-26$.

Shi, F., Ma, Y., Ma, J., Wang, P., \& Sun, W. (2013). Preparation and characterization of PVDF/TiO2 hybrid membranes with ionic liquid modified nano-TiO2 particles. Journal of Membrane Science, 427, 259-269.

Shi, L., Wang, R., Cao, Y., Feng, C., Liang, D. T., \& Tay, J. H. (2007). Fabrication of poly (vinylidene fluoride-co-hexafluropropylene)(PVDF-HFP) asymmetric microporous hollow fiber membranes. Journal of Membrane Science, 305(1), $215-225$.

Shi, X., Field, R., \& Hankins, N. (2011). Review of fouling by mixed feeds in membrane filtration applied to water purification. Desalination and Water Treatment, 35(1-3), 68-81. doi:10.5004/dwt.2011.3131

Shirazi, S., Lin, C.-J., \& Chen, D. (2010). Inorganic fouling of pressure-driven membrane processes - a critical review. Desalination, 250(1), 236-248.

Singh, G., Joyce, E. M., Beddow, J., \& Mason, T. J. (2012). Evaluation of antibacterial activity of $\mathrm{ZnO}$ nanoparticles coated sonochemically onto textile fabrics. The Journal of Microbiology, Biotechnology and Food Sciences, 2(1), 106.

Singh, N., Nelson, B., Scanlan, L., Coskun, E., Jaruga, P., \& Doak, S. (2017). Exposure to Engineered Nanomaterials: Impact on DNA Repair Pathways. International Journal of Molecular Sciences, 18(7), 1515.

Singh, S., Khulbe, K., Matsuura, T., \& Ramamurthy, P. (1998). Membrane characterization by solute transport and atomic force microscopy. Journal of Membrane Science, 142(1), 111-127.

Singh, V., Joung, D., Zhai, L., Das, S., Khondaker, S. I., \& Seal, S. (2011). Graphene based materials: Past, present and future. Progress in Materials Science, 56(8), $1178-1271$.

Sofiah, H., Nora'aini, A., \& Marinah, M. (2010). The Influence of Polymer Concentration on Performance and Morphology of Asymmetric Ultrafiltration Membrane for Lysozyme Separation. Journal of Applied Sciences(Faisalabad), 10(24), 3325-3330. 
Somanathan, T., Prasad, K., Ostrikov, K. K., Saravanan, A., \& Krishna, V. M. (2015). Graphene oxide synthesis from agro waste. Nanomaterials, 5(2), 826-834.

Song, J. Y., \& Kim, B. S. (2009). Rapid biological synthesis of silver nanoparticles using plant leaf extracts. Bioprocess Biosyst Eng, 32(1), 79-84.

Sotiriou, G. A., \& Pratsinis, S. E. (2010). Antibacterial activity of nanosilver ions and particles. Environmental Science \& Technology, 44(14), 5649-5654.

Strathmann, H., Giorno, L., \& Drioli, E. (2011). Introduction to membrane science and technology (Vol. 544): Wiley-VCH Verlag \& Company.

Subramani, A., \& Hoek, E. (2008). Direct observation of initial microbial deposition onto reverse osmosis and nanofiltration membranes. Journal of Membrane Science, 319(1-2), 111-125.

Subramanian, A. S., Tey, J. N., Zhang, L., Ng, B. H., Roy, S., \& Wei, J. (2016). Synergistic bond strengthening in epoxy adhesives using polydopamine/MWCNT hybrids. Polymer, 82, 285-294.

Susanto, H., Arafat, H., Janssen, E. M., \& Ulbricht, M. (2008). Ultrafiltration of polysaccharide-protein mixtures: elucidation of fouling mechanisms and fouling control by membrane surface modification. Separation and Purification Technology, 63(3), 558-565.

Tang, C. Y., Kwon, Y.-N., \& Leckie, J. O. (2007). Fouling of reverse osmosis and nanofiltration membranes by humic acid - effects of solution composition and hydrodynamic conditions. Journal of Membrane Science, 290(1), 86-94.

Tang, S. C., \& Lo, I. M. (2013). Magnetic nanoparticles: essential factors for sustainable environmental applications. Water Research, 47(8), 2613-2632.

Tarabara, V. V. (2014). Multifunctional Nanomaterial-Enabled Membranes for Water Treatment. Nanotechnology Applications for Clean Water, 5, 155-171.

Thomas, T. E., Aani, S. A., Oatley-Radcliffe, D. L., Williams, P. M., \& Hilal, N. (2017). Laser Doppler Electrophoresis and electro-osmotic flow mapping: A novel methodology for the determination of membrane surface zeta potential. Journal of Membrane Science, 523, 524-532.

Tiwari, J. N., Tiwari, R. N., \& Kim, K. S. (2012). Zero-dimensional, one-dimensional, two-dimensional and three-dimensional nanostructured materials for advanced electrochemical energy devices. Progress in Materials Science, 57(4), 724-803.

Tolaymat, T. M., El Badawy, A. M., Genaidy, A., Scheckel, K. G., Luxton, T. P., \& Suidan, M. (2010). An evidence-based environmental perspective of manufactured silver nanoparticle in syntheses and applications: a systematic review and critical appraisal of peer-reviewed scientific papers. Science of the Total Environment, 408(5), 999-1006.

Travessa, D. N., Silva, F. S. d., Cristovan, F. H., Jorge Jr, A. M., \& Cardoso, K. R. (2014). Ag ion decoration for surface modifications of multi-walled carbon nanotubes. Materials Research, 17(3), 687-693.

Trejo, N. K., \& Frey, M. (2015). A comparative study on electrosprayed, layer-by-layer, and chemically grafted nanomembranes loaded with iron oxide nanoparticles. Journal of applied polymer science, 132(41). 
Tu, S.-C., Ravindran, V., \& Pirbazari, M. (2005). A pore diffusion transport model for forecasting the performance of membrane processes. Journal of Membrane Science, 265(1), 29-50.

Ulbricht, M. (2006). Advanced functional polymer membranes. Polymer, 47(7), 22172262.

Upen, J., Barwada, S. J., Coker, S., \& Terry, A. (2000). Winning the battle against biofouling of reverse osmosis membranes. Desalination Water Reuse, 10(2), 53-54.

Vairavapandian, D., Vichchulada, P., \& Lay, M. D. (2008). Preparation and modification of carbon nanotubes: Review of recent advances and applications in catalysis and sensing. Analytica chimica acta, 626(2), 119-129.

Van de Lisdonk, C., Van Paassen, J., \& Schippers, J. (2000). Monitoring scaling in nanofiltration and reverse osmosis membrane systems. Desalination, 132(1-3), $101-108$

Van de Witte, P., Dijkstra, P., Van den Berg, J., \& Feijen, J. (1996). Phase separation processes in polymer solutions in relation to membrane formation. Journal of Membrane Science, 117(1), 1-31.

Van der Bruggen, B. (2009). Chemical modification of polyethersulfone nanofiltration membranes: A review. Journal of applied polymer science, 114(1), 630-642.

Van der Bruggen, B. (2012). The Separation Power of Nanotubes in Membranes: A Review. ISRN Nanotechnology, 2012, 1-17.

Van der Bruggen, B., Mänttäri, M., \& Nyström, M. (2008). Drawbacks of applying nanofiltration and how to avoid them: a review. Separation and Purification Technology, 63(2), 251-263.

Vance, M. E., Kuiken, T., Vejerano, E. P., McGinnis, S. P., Hochella, M. F., Jr., Rejeski, D., \& Hull, M. S. (2015). Nanotechnology in the real world: Redeveloping the nanomaterial consumer products inventory. Beilstein Journal of Nanotechnol, 6, 1769-1780.

Vatanpour, V., Madaeni, S. S., Khataee, A. R., Salehi, E., Zinadini, S., \& Monfared, H. A. (2012). TiO2 embedded mixed matrix PES nanocomposite membranes: Influence of different sizes and types of nanoparticles on antifouling and performance. Desalination, 292, 19-29.

Vatanpour, V., Madaeni, S. S., Moradian, R., Zinadini, S., \& Astinchap, B. (2011). Fabrication and characterization of novel antifouling nanofiltration membrane prepared from oxidized multiwalled carbon nanotube/polyethersulfone nanocomposite. Journal of Membrane Science, 375(1-2), 284-294.

Vázquez, E., \& Prato, M. (2009). Carbon nanotubes and microwaves: interactions, responses, and applications. ACS nano, 3(12), 3819-3824.

Vickery, J. L., Patil, A. J., \& Mann, S. (2009). Fabrication of Graphene-Polymer Nanocomposites With Higher-Order Three-Dimensional Architectures. Advanced Materials, 21(21), 2180-2184.

Vinayan, B. P., Nagar, R., Raman, V., Rajalakshmi, N., Dhathathreyan, K. S., \& Ramaprabhu, S. (2012). Synthesis of graphene-multiwalled carbon nanotubes 
hybrid nanostructure by strengthened electrostatic interaction and its lithium ion battery application. Journal of Materials Chemistry, 22(19), 9949.

Vinci, R. P., \& Vlassak, J. J. (1996). Mechanical behavior of thin films. Annual Review of Materials Science, 26(1), 431-462.

Vrijenhoek, E. M., Hong, S., \& Elimelech, M. (2001). Influence of membrane surface properties on initial rate of colloidal fouling of reverse osmosis and nanofiltration membranes. Journal of Membrane Science, 188(1), 115-128.

Vuković, G., Marinković, A., Obradović, M., Radmilović, V., Čolić, M., Aleksić, R., \& Uskoković, P. S. (2009). Synthesis, characterization and cytotoxicity of surface amino-functionalized water-dispersible multi-walled carbon nanotubes. Applied Surface Science, 255(18), 8067-8075.

W.R. Bowen , J. I. C., A. Hermindez . (1995). Steps of membrane blocking in flux decline during protein microfiltration. Journal of Membrane Science, 101( ), $153-165$

Wan, L.-S., Xu, Z.-K., \& Wang, Z.-G. (2006). Leaching of PVP from polyacrylonitrile/PVP blending membranes: A comparative study of asymmetric and dense membranes. Journal of Polymer Science Part B: Polymer Physics, 44(10), 1490-1498.

Wang, C., Zhao, S., \& Wei, Y. (2012). Hydrophilic Modification of Microporous Polysulfone Membrane via Surface-Initiated Atom Transfer Radical Polymerization and Hydrolysis of Poly (glycidylmethacrylate). Chinese Journal of Chemistry, 30(10), 2473-2482.

Wang, D., Li, K., \& Teo, W. (1995). Relationship between mass ratio of nonsolventadditive to solvent in membrane casting solution and its coagulation value. Journal of Membrane Science, 98(3), 233-240.

Wang, D., Li, K., \& Teo, W. (2000). Porous PVDF asymmetric hollow fiber membranes prepared with the use of small molecular additives. Journal of Membrane Science, 178(1), 13-23.

Wang, D., Li, Z.-C., \& Chen, L. (2006a). Templated synthesis of single-walled carbon nanotube and metal nanoparticle assemblies in solution. Journal of the American Chemical Society, 128(47), 15078-15079.

Wang, F., \& Tarabara, V. V. (2008). Pore blocking mechanisms during early stages of membrane fouling by colloids. Journal of colloid and interface science, 328(2), 464-469.

Wang, L., Song, X., Wang, T., Wang, S., Wang, Z., \& Gao, C. (2015a). Fabrication and characterization of polyethersulfone/carbon nanotubes (PES/CNTs) based mixed matrix membranes (MMMs) for nanofiltration application. Applied Surface Science, 330, 118-125.

Wang, M., Wu, L., \& Gao, C. (2006b). The influence of phase inversion process modified by chemical reaction on membrane properties and morphology. Journal of Membrane Science, 270(1-2), 154-161.

Wang, P., Ma, J., Shi, F., Ma, Y., Wang, Z., \& Zhao, X. (2013). Behaviors and Effects of Differing Dimensional Nanomaterials in Water Filtration Membranes 
through the Classical Phase Inversion Process: A Review. Industrial \& Engineering Chemistry Research, 52(31), 10355-10363.

Wang, W., Serp, P., Kalck, P., Silva, C. G., \& Faria, J. L. (2008). Preparation and characterization of nanostructured MWCNT-TiO 2 composite materials for photocatalytic water treatment applications. Materials Research Bulletin, 43(4), 958-967.

Wang, X., Zhan, C., Kong, B., Zhu, X., Liu, J., Xu, W., . . Wang, H. (2015b). Selfcurled coral-like gamma-A12O3 nanoplates for use as an adsorbent. Journal of colloid and interface science, 453, 244-251.

Wang, Y.-Q., Su, Y.-L., Ma, X.-L., Sun, Q., \& Jiang, Z.-Y. (2006c). Pluronic polymers and polyethersulfone blend membranes with improved fouling-resistant ability and ultrafiltration performance. Journal of Membrane Science, 283(1-2), 440447.

Warwick, M. E. A., Kaunisto, K., Barreca, D., Carraro, G., Gasparotto, A., Maccato, C., . . . Turner, S. (2015). Vapor phase processing of $\alpha-\mathrm{Fe} 2 \mathrm{O} 3$ photoelectrodes for water splitting: an insight into the structure/property interplay. ACS applied materials \& interfaces, 7(16), 8667-8676.

Watson, B., \& Hornburg, C. (1989). Low-energy membrane nanofiltration for removal of color, organics and hardness from drinking water supplies. Desalination, $72(1), 11-22$.

Welderfael, T., Pattabi, M., \& Pattabi, R. M. (2016). Photocatalytic activity of Ag-N co-doped $\mathrm{ZnO}$ nanorods under visible and solar light irradiations for $\mathrm{MB}$ degradation. Journal of Water Process Engineering, 14, 117-123.

Wienk, I., Boom, R., Beerlage, M., Bulte, A., Smolders, C., \& Strathmann, H. (1996). Recent advances in the formation of phase inversion membranes made from amorphous or semi-crystalline polymers. Journal of Membrane Science, 113(2), 361-371.

Wu, H., Mansouri, J., \& Chen, V. (2013). Silica nanoparticles as carriers of antifouling ligands for PVDF ultrafiltration membranes. Journal of Membrane Science, 433, 135-151.

Wu, H., Tang, B., \& Wu, P. (2010). MWNTs/polyester thin film nanocomposite membrane: an approach to overcome the trade-off effect between permeability and selectivity. The Journal of Physical Chemistry C, 114(39), 16395-16400.

Wu, H., Wu, G., \& Wang, L. (2015). Peculiar porous $\alpha$-Fe 2 O 3, $\gamma$-Fe 2 O 3 and Fe 3 O 4 nanospheres: facile synthesis and electromagnetic properties. Powder Technology, 269, 443-451.

Wu, M.-B., Lv, Y., Yang, H.-C., Liu, L.-F., Zhang, X., \& Xu, Z.-K. (2016). Thin film composite membranes combining carbon nanotube intermediate layer and microfiltration support for high nanofiltration performances. Journal of Membrane Science, 515, 238-244.

Xia, S., \& Ni, M. (2015). Preparation of poly(vinylidene fluoride) membranes with graphene oxide addition for natural organic matter removal. Journal of Membrane Science, 473, 54-62. 
Xie, X., Mai, Y., \& Zhou, X. (2005). Dispersion and alignment of carbon nanotubes in polymer matrix: A review. Materials Science and Engineering, 49(4), 89-112.

Xiu, Z. M., Ma, J., \& Alvarez, P. J. (2011). Differential effect of common ligands and molecular oxygen on antimicrobial activity of silver nanoparticles versus silver ions. Environmental Science and Technology, 45(20), 9003-9008.

Xu, G.-R., Wang, S.-H., Zhao, H.-L., Wu, S.-B., Xu, J.-M., Li, L., \& Liu, X.-Y. (2015). Layer-by-layer (LBL) assembly technology as promising strategy for tailoring pressure-driven desalination membranes. Journal of Membrane Science, 493, 428-443.

Xu, P., Zeng, G. M., Huang, D. L., Feng, C. L., Hu, S., Zhao, M. H., . . . Liu, Z. F. (2012). Use of iron oxide nanomaterials in wastewater treatment: a review. Science of the Total Environment, 424, 1-10.

Xu, Z.-1., Yu, L.-y., \& Han, L.-f. (2009). Polymer-nanoinorganic particles composite membranes: a brief overview. Frontiers of Chemical Engineering in China, 3(3), 318-329.

Xu, Z., Wu, T., Shi, J., Teng, K., Wang, W., Ma, M., . . Fan, J. (2016a). Photocatalytic antifouling PVDF ultrafiltration membranes based on synergy of graphene oxide and TiO 2 for water treatment. Journal of Membrane Science, 520, 281293.

Xu, Z., Ye, S., Zhang, G., Li, W., Gao, C., Shen, C., \& Meng, Q. (2016b). Antimicrobial polysulfone blended ultrafiltration membranes prepared with $\mathrm{Ag} / \mathrm{Cu} 2 \mathrm{O}$ hybrid nanowires. Journal of Membrane Science, 509, 83-93.

Xu, Z., Zhang, J., Shan, M., Li, Y., Li, B., Niu, J., . . Qian, X. (2014). Organosilanefunctionalized graphene oxide for enhanced antifouling and mechanical properties of polyvinylidene fluoride ultrafiltration membranes. Journal of Membrane Science, 458, 1-13.

Xue, B., Chen, P., Hong, Q., Lin, J., \& Tan, K. L. (2001). Growth of Pd, Pt, Ag and Au nanoparticles on carbon nanotubes. Journal of Materials Chemistry, 11(9), 2378-2381.

Yan, L., Li, Y. S., \& Xiang, C. B. (2005). Preparation of poly (vinylidene fluoride)(pvdf) ultrafiltration membrane modified by nano-sized alumina (Al 2 O 3 ) and its antifouling research. Polymer, 46(18), 7701-7706.

Yang, J., Ni, K., Wei, D., \& Ren, Y. (2015). One-step purification and immobilization of his-tagged protein via Ni2+-functionalized Fe3O4@ polydopamine magnetic nanoparticles. Biotechnology and bioprocess engineering, 20(5), 901-907.

Yin, J., Yang, Y., Hu, Z., \& Deng, B. (2013). Attachment of silver nanoparticles (AgNPs) onto thin-film composite (TFC) membranes through covalent bonding to reduce membrane biofouling. Journal of Membrane Science, 441, 73-82.

Yin, J., Zhu, G., \& Deng, B. (2016). Graphene oxide (GO) enhanced polyamide (PA) thin-film nanocomposite (TFN) membrane for water purification. Desalination, 379, 93-101.

Young, R. J., Kinloch, I. A., Gong, L., \& Novoselov, K. S. (2012). The mechanics of graphene nanocomposites: A review. Composites Science and Technology, 72(12), 1459-1476. 
Yu, H.-Y., Xie, Y.-J., Hu, M.-X., Wang, J.-L., Wang, S.-Y., \& Xu, Z.-K. (2005a). Surface modification of polypropylene microporous membrane to improve its antifouling property in MBR: CO 2 plasma treatment. Journal of Membrane Science, 254(1), 219-227.

Yu, H., Xie, Y., Hu, M., Wang, J., Wang, S., \& Xu, Z. (2005b). Surface modification of polypropylene microporous membrane to improve its antifouling property in MBR: CO plasma treatment. Journal of Membrane Science, 254(1-2), 219-227.

Yu, L., Zhang, Y., Zhang, B., Liu, J., Zhang, H., \& Song, C. (2013). Preparation and characterization of HPEI-GO/PES ultrafiltration membrane with antifouling and antibacterial properties. Journal of Membrane Science, 447, 452-462.

Yu, S., Zuo, X., Bao, R., Xu, X., Wang, J., \& Xu, J. (2009). Effect of SiO2 nanoparticle addition on the characteristics of a new organic-inorganic hybrid membrane. Polymer, 50(2), 553-559.

Yu, Y., Jimmy, C. Y., Yu, J.-G., Kwok, Y.-C., Che, Y.-K., Zhao, J.-C., . . Wong, P.K. (2005c). Enhancement of photocatalytic activity of mesoporous TiO 2 by using carbon nanotubes. Applied Catalysis A: General, 289(2), 186-196.

Yu, Y., Shapter, J. G., Popelka-Filcoff, R., Bennett, J. W., \& Ellis, A. V. (2014). Copper removal using bio-inspired polydopamine coated natural zeolites. Journal of hazardous materials, 273, 174-182.

Yuan, W., Jiang, G., Che, J., Qi, X., Xu, R., Chang, M. W., . . . Chan-Park, M. B. (2008). Deposition of silver nanoparticles on multiwalled carbon nanotubes grafted with hyperbranched poly (amidoamine) and their antimicrobial effects. The Journal of Physical Chemistry C, 112(48), 18754-18759.

Yuan, W., \& Zydney, A. L. (2000). Humic acid fouling during ultrafiltration. Environmental Science \& Technology, 34(23), 5043-5050.

Yudianti, R., Onggo, H., Sudirman, Y. S., Iwata, T., \& Azuma, J.-i. (2011). Analysis of functional group sited on multi-wall carbon nanotube surface. Open Materials Science Journal, 5, 242-247.

Zamudio, A., Elías, A. L., Rodríguez-Manzo, J. A., López-Urías, F., RodríguezGattorno, G., Lupo, F., . . . Díaz, D. (2006). Efficient Anchoring of Silver Nanoparticles on N-Doped Carbon Nanotubes. Small, 2(3), 346-350.

Zazouli, M. A., Nasseri, S., \& Ulbricht, M. (2010). Fouling effects of humic and alginic acids in nanofiltration and influence of solution composition. Desalination, 250(2), 688-692.

Zeman, L. J., \& Zydney, A. (1996). Microfiltration and ultrafiltration: principles and applications. New York, NY, USA: Marcel Dekker, Inc. .

Zhang, J., Xu, Z., Mai, W., Min, C., Zhou, B., Shan, M., .. . Qian, X. (2013a). Improved hydrophilicity, permeability, antifouling and mechanical performance of PVDF composite ultrafiltration membranes tailored by oxidized low-dimensional carbon nanomaterials. Journal of Materials Chemistry A, 1(9), 3101-3111.

Zhang, J., Xu, Z., Shan, M., Zhou, B., Li, Y., Li, B., . . Qian, X. (2013b). Synergetic effects of oxidized carbon nanotubes and graphene oxide on fouling control and anti-fouling mechanism of polyvinylidene fluoride ultrafiltration membranes. Journal of Membrane Science, 448, 81-92. 
Zhang, L., Yuan, F., Zhang, X., \& Yang, L. (2011). Facile synthesis of flower like copper oxide and their application to hydrogen peroxide and nitrite sensing. Chem Central J, 5, 75-83.

Zhang, M., Zhang, K., De Gusseme, B., \& Verstraete, W. (2012). Biogenic silver nanoparticles (bio-Ag 0) decrease biofouling of bio-Ag 0/PES nanocomposite membranes. Water Res, 46(7), 2077-2087.

Zhang, R.-X., Braeken, L., Luis, P., Wang, X.-L., \& Van der Bruggen, B. (2013c). Novel binding procedure of $\mathrm{TiO} 2$ nanoparticles to thin film composite membranes via self-polymerized polydopamine. Journal of Membrane Science, 437, 179-188.

Zhang, W., Shi, Z., Zhang, F., Liu, X., Jin, J., \& Jiang, L. (2013d). Superhydrophobic and superoleophilic PVDF membranes for effective separation of water-in-oil emulsions with high flux. Advance Materials, 25(14), 2071-2076.

Zhang, X., Zeng, G., Tian, J., Wan, Q., Huang, Q., Wang, K., . . Wei, Y. (2015). PEGylation of carbon nanotubes via mussel inspired chemistry: preparation, characterization and biocompatibility evaluation. Applied Surface Science, 351, 425-432.

Zhao, C., Xue, J., Ran, F., \& Sun, S. (2013). Modification of polyethersulfone membranes - A review of methods. Progress in Materials Science, 58(1), 76150 .

Zhao, F., Qian, W., Li, M., Li, W., Chen, L., Zhong, F., . . Dong, C. (2017). Directly grown carbon nanotube based hybrid electrodes with enhanced thermo-cell performances. RSC Advances, 7(38), 23890-23895.

Zhao, F. Y., Ji, Y. L., Weng, X. D., Mi, Y. F., Ye, C. C., An, Q. F., \& Gao, C. J. (2016). High-Flux Positively Charged Nanocomposite Nanofiltration Membranes Filled with Poly(dopamine) Modified Multiwall Carbon Nanotubes. ACS Applied Materials and Interfaces, 8(10), 6693-6700.

Zhao, H., Qiu, S., Wu, L., Zhang, L., Chen, H., \& Gao, C. (2014). Improving the performance of polyamide reverse osmosis membrane by incorporation of modified multi-walled carbon nanotubes. Journal of Membrane Science, 450, 249-256.

Zhao, J., Shi, Q., Luan, S., Song, L., Yang, H., Shi, H., . . Stagnaro, P. (2011a). Improved biocompatibility and antifouling property of polypropylene nonwoven fabric membrane by surface grafting zwitterionic polymer. Journal of Membrane Science, 369(1-2), 5-12.

Zhao, S., Wang, Z., Wei, X., Tian, X., Wang, J., Yang, S., \& Wang, S. (2011b). Comparison study of the effect of PVP and PANI nanofibers additives on membrane formation mechanism, structure and performance. Journal of Membrane Science, 385, 110-122.

Zhu, X., \& Elimelech, M. (1997). Colloidal fouling of reverse osmosis membranes: measurements and fouling mechanisms. Environmental Science \& Technology, 31(12), 3654-3662.

Zinadini, S., Zinatizadeh, A. A., Rahimi, M., Vatanpour, V., \& Zangeneh, H. (2014). Preparation of a novel antifouling mixed matrix PES membrane by embedding graphene oxide nanoplates. Journal of Membrane Science, 453, 292-301. 
Zodrow, K., Brunet, L., Mahendra, S., Li, D., Zhang, A., Li, Q., \& Alvarez, P. J. (2009). Polysulfone ultrafiltration membranes impregnated with silver nanoparticles show improved biofouling resistance and virus removal. Water Research, 43(3), 715-723. 